University of Tennessee Health Science Center UTHSC Digital Commons

$12-2009$

\title{
Chitosan/Ellagic Acid Composite Materials for Local Cancer Therapy
}

Sung Woo Kim

University of Tennessee Health Science Center

Follow this and additional works at: https://dc.uthsc.edu/dissertations

Part of the Therapeutics Commons

\section{Recommended Citation}

Kim, Sung Woo, "Chitosan/Ellagic Acid Composite Materials for Local Cancer Therapy" (2009). Theses and Dissertations (ETD). Paper 131. http://dx.doi.org/10.21007/etd.cghs.2009.0163.

This Dissertation is brought to you for free and open access by the College of Graduate Health Sciences at UTHSC Digital Commons. It has been accepted for inclusion in Theses and Dissertations (ETD) by an authorized administrator of UTHSC Digital Commons. For more information, please contact jwelch30@uthsc.edu. 


\title{
Chitosan/Ellagic Acid Composite Materials for Local Cancer Therapy
}

\begin{abstract}
Current advances in the drug delivery have improved the therapeutic efficacy of the drug and minimized risks of side effects associated with toxicity of the drug. Implantable polymeric delivery system has gained increasing attentions for controlled drug release and localized treatments. In comparison to conventional chemotherapy, polymeric delivery systems are implantable at a local targeted site and biodegradable after a sufficient therapeutic span. The objectives of this project were to fabricate and characterize an implantable polymeric vehicle for a local chemotherapy and investigate its biological properties against cancer cells including human WM115 melanoma, human U87 glioblastoma, and rat C6 glioma cells in vitro andin vivo.
\end{abstract}

In this study, a natural chitosan polymer was employed as a drug vehicle and ellagic acid (EA), a naturally occurring phenolic compound, was incorporated as a therapeutic agent. The chitosan/ellagic acid composite films were developed by combining $1 \%(\mathrm{w} / \mathrm{v})$ chitosan solution with different concentrations $(0.05,0.1,0.5,1$, or $20 \%(\mathrm{w} / \mathrm{v}))$ of ellagic acid for a local chemotherapy. Characterization of composite films was performed on chemical structure, crystallinity, surface morphology, degradation behavior, and release profile. Cancer cell activity on the composite films was evaluated through direct and indirect cell culture using MTS assay. Anti-cancer mechanism of the composite films against cancer cells was investigated using apoptosis assay, caspase- 3 activation, western blot for $p 53$, and anti-angiogenesis assays. In the in vivo study, an animal subcutaneous model was used to assess the anti-tumor effect of the composite film on rat $\mathrm{C} 6$ glioma. Treatments were initiated by implanting the composite films onto the tumor. The tumor growth was monitored by measuring tumor volume using a caliper, an ultrasound machine, and an optical imaging system.

The chitosan/ellagic acid composite films exhibited increase in amide and ester linkages, diffraction peaks of the crystallized ellagic acid, enhanced surface roughness, and hydrophilicity with increasing concentration of ellagic acid. The composite films degraded enzymatically, indicated by at least a 5 times higher concentration of free amino groups in the incubation medium at 3 weeks compared with 1 day. They also displayed a sustained slow release of ellagic acid in vitro for 3 weeks incubation. Anti-cancer activity of the composite films was ellagic acid concentration dependent by inducing apoptosis of cancer cells and suppressing angiogenesis. Significant inhibitory effect $(p<0.05)$ was found in the composite films containing $0.5 \%(\mathrm{w} / \mathrm{v})$ of ellagic acid or higher compared with other groups. Study of a rat $\mathrm{C} 6 \mathrm{glioma}$ model demonstrated that the composite film (Ch/EA20) significantly inhibited tumor growth compared with control groups in vivo. Tumor volume increase in Ch/EA20 group was 9 times lower than that in control groups at 3 weeks observation by measuring a caliper. No severe weight loss ( $>10 \%$ wt.) was observed from all groups. Histology observation indicated no evidence of severe toxicity surrounding the composite films. The high efficacy and low toxicity of the composite film was attributed to the slow release and localized effect of ellagic acid.

In order to further improve the delivery method and efficacy, chitosan based injectable hydrogel was developed for a local administration of ellagic acid to avoid surgical complications. Studies of the chitosan gel were performed with regard to chemical structure, surface morphology, viscoelasticity, release profile, and degradation behavior. Biocompatibility and anti-cancer activity on chitosan gel delivery system were examined. The results showed that the injectable chitosan liquid formulation underwent thermal gelation at body temperature via hydrophobic interactions using $\beta$-glycerophosphate salt ( $\beta$-GP). Sol-gel transition was dependent on final $\mathrm{pH}$ values of the chitosan/ $\beta$-GP solution and temperature. Dialysis of chitosan solution reduced the $\beta$-GP needed to reach $\mathrm{pH} 7.2$, resulting in 4 times higher cell viability than undialyzed chitosan gel at 3 days culture. This result indicates improved biocompatibility of the delivery system. The chitosan/ $\beta$-GP gels were enzymatically degradable for 3 weeks incubation and 
inhibited cancer cell growth in vitro in an ellagic acid concentration dependent manner. The significant inhibitory effect $(p<0.05)$ was found in the gel containing $1 \%(\mathrm{w} / \mathrm{v})$ of ellagic acid compared with other groups. Viability of U87 cells and C6 cells cultured on chitosan gels containing $1 \%(\mathrm{w} / \mathrm{v})$ of ellagic acid were lower than the same cells on chitosan gels at 3 days incubation by 3.8 times and 6.5 times, respectively.

This research has demonstrated that the chitosan/ellagic acid delivery system is a promising biomaterial for a local cancer treatment. This study has also suggested a potential strategy with higher efficacy and lower toxicity to treat tumors by the combination of naturally based biopolymers such as chitosan and phenolic compounds such as ellagic acid. This study provides some rationale for further investigation of implantable polymeric delivery system.

\section{Document Type}

Dissertation

\section{Degree Name}

Doctor of Philosophy (PhD)

\section{Program}

Biomedical Engineering and Imaging

\section{Research Advisor}

Denis J. DiAngelo, Ph.D.

\section{Keywords}

Apoptosis, Biocompatibility, Cancer, Chitosan, Ellagic acid, Local drug delivery

\section{Subject Categories}

Analytical, Diagnostic and Therapeutic Techniques and Equipment | Medicine and Health Sciences | Therapeutics 


\title{
CHITOSAN/ELLAGIC ACID COMPOSITE MATERIALS FOR LOCAL CANCER THERAPY
}

\author{
A Dissertation \\ Presented for \\ The Graduate Studies Council \\ The University of Tennessee \\ Health Science Center
}

\begin{abstract}
In Partial Fulfillment
Of the Requirements for the Degree

Doctor of Philosophy

In the Joint Graduate Program in Biomedical Engineering and Imaging

From The University of Tennessee

and

The University of Memphis
\end{abstract}

By

Sung Woo Kim

December 2009 
Portions of Chapter 3 (C) 2008 by Wiley Periodicals.

Portions of Chapter 4 (C) 2009 by Elsevier.

All other material (C) 2009 by Sung Woo Kim. 


\section{DEDICATION}

This dissertation is dedicated to my precious parents, Heung Soo Kim and Yang Oak Song, and to my lovely wife, Ji Hyun Choi, for their endless love and continuous support. 


\section{ACKNOWLEDGEMENTS}

I would like to express my deepest gratitude to my advisor, Dr. Yunzhi "Peter" Yang for providing me with an opportunity to work on this research. His guidance, support, and constant help have made this dissertation possible. I would also like to thank my committee members, Dr. Denis J. DiAngelo, Dr. Mostafa W. Gaber, Dr. Warren O. Haggard, Dr. Joel D. Bumgardner, Dr. Satoru K. Nishimoto and Dr. Xin A. Zhang for their excellent advice, experience, and encouragement over the years. Their cooperation and suggestions have improved the quality of my dissertation considerably.

I would like to thank the members of Dr. Yang's lab, Dr. Yongxing Liu, Dr. Joong Hyun Kim, Daniel Young and Dr. Yunqing Kang for their support and wonderful company. I am also grateful to my friends and colleagues including Bharathi Gogula, Janice Zawaski, Feng Zhang, and Mekel Richardson for their suggestions and assistance.

I would also like to thank the journals, Biomaterials and Journal of Biomedical Material Research Part B: Applied Biomaterial for granting permission to use my published works in this dissertation.

My deepest appreciation goes to my parents and my wife. No words would be enough to thank their patience, encouragement, and support throughout the years. They have been an inexhaustible source of love and inspiration for all my life. 


\begin{abstract}
Current advances in the drug delivery have improved the therapeutic efficacy of the drug and minimized risks of side effects associated with toxicity of the drug. Implantable polymeric delivery system has gained increasing attentions for controlled drug release and localized treatments. In comparison to conventional chemotherapy, polymeric delivery systems are implantable at a local targeted site and biodegradable after a sufficient therapeutic span. The objectives of this project were to fabricate and characterize an implantable polymeric vehicle for a local chemotherapy and investigate its biological properties against cancer cells including human WM115 melanoma, human U87 glioblastoma, and rat C6 glioma cells in vitro and in vivo.

In this study, a natural chitosan polymer was employed as a drug vehicle and ellagic acid (EA), a naturally occurring phenolic compound, was incorporated as a therapeutic agent. The chitosan/ellagic acid composite films were developed by combining $1 \%(\mathrm{w} / \mathrm{v})$ chitosan solution with different concentrations $(0.05,0.1,0.5,1$, or $20 \%(\mathrm{w} / \mathrm{v}))$ of ellagic acid for a local chemotherapy. Characterization of composite films was performed on chemical structure, crystallinity, surface morphology, degradation behavior, and release profile. Cancer cell activity on the composite films was evaluated through direct and indirect cell culture using MTS assay. Anti-cancer mechanism of the composite films against cancer cells was investigated using apoptosis assay, caspase-3 activation, western blot for $\mathrm{p} 53$, and anti-angiogenesis assays. In the in vivo study, an animal subcutaneous model was used to assess the anti-tumor effect of the composite film on rat C6 glioma. Treatments were initiated by implanting the composite films onto the tumor. The tumor growth was monitored by measuring tumor volume using a caliper, an ultrasound machine, and an optical imaging system.

The chitosan/ellagic acid composite films exhibited increase in amide and ester linkages, diffraction peaks of the crystallized ellagic acid, enhanced surface roughness, and hydrophilicity with increasing concentration of ellagic acid. The composite films degraded enzymatically, indicated by at least a 5 times higher concentration of free amino groups in the incubation medium at 3 weeks compared with 1 day. They also displayed a sustained slow release of ellagic acid in vitro for 3 weeks incubation. Anti-cancer activity of the composite films was ellagic acid concentration dependent by inducing apoptosis of cancer cells and suppressing angiogenesis. Significant inhibitory effect $(p<0.05)$ was found in the composite films containing $0.5 \%(\mathrm{w} / \mathrm{v})$ of ellagic acid or higher compared with other groups. Study of a rat C6 glioma model demonstrated that the composite film (Ch/EA20) significantly inhibited tumor growth compared with control groups in vivo. Tumor volume increase in $\mathrm{Ch} / \mathrm{EA} 20$ group was 9 times lower than that in control groups at 3 weeks observation by measuring a caliper. No severe weight loss $(>10 \%$ wt.) was observed from all groups. Histology observation indicated no evidence of severe toxicity surrounding the composite films. The high efficacy and low toxicity of the composite film was attributed to the slow release and localized effect of ellagic acid.
\end{abstract}

In order to further improve the delivery method and efficacy, chitosan based 
injectable hydrogel was developed for a local administration of ellagic acid to avoid surgical complications. Studies of the chitosan gel were performed with regard to chemical structure, surface morphology, viscoelasticity, release profile, and degradation behavior. Biocompatibility and anti-cancer activity on chitosan gel delivery system were examined. The results showed that the injectable chitosan liquid formulation underwent thermal gelation at body temperature via hydrophobic interactions using $\beta$ glycerophosphate salt ( $\beta$-GP). Sol-gel transition was dependent on final $\mathrm{pH}$ values of the chitosan/ $\beta$-GP solution and temperature. Dialysis of chitosan solution reduced the $\beta$-GP needed to reach $\mathrm{pH} 7.2$, resulting in 4 times higher cell viability than undialyzed chitosan gel at 3 days culture. This result indicates improved biocompatibility of the delivery system. The chitosan/ $\beta$-GP gels were enzymatically degradable for 3 weeks incubation and inhibited cancer cell growth in vitro in an ellagic acid concentration dependent manner. The significant inhibitory effect $(p<0.05)$ was found in the gel containing $1 \%$ (w/v) of ellagic acid compared with other groups. Viability of U87 cells and C6 cells cultured on chitosan gels containing $1 \%(\mathrm{w} / \mathrm{v})$ of ellagic acid were lower than the same cells on chitosan gels at 3 days incubation by 3.8 times and 6.5 times, respectively.

This research has demonstrated that the chitosan/ellagic acid delivery system is a promising biomaterial for a local cancer treatment. This study has also suggested a potential strategy with higher efficacy and lower toxicity to treat tumors by the combination of naturally based biopolymers such as chitosan and phenolic compounds such as ellagic acid. This study provides some rationale for further investigation of implantable polymeric delivery system. 


\section{TABLE OF CONTENTS}

CHAPTER 1. INTRODUCTION 1

1.1 Local Drug Delivery Systems 1

1.2 Implantable Polymeric Delivery System 4

1.2.1 Biodegradable Polymers 4

1.2.2 Natural Chitosan Polymer $\quad 7$

1.3 Dietary Polyphenols 9

$\begin{array}{ll}\text { 1.3.1 Chemical Structure } & 9\end{array}$

$\begin{array}{lr}\text { 1.3.2 Biological Properties } & 10\end{array}$

$\begin{array}{lll}1.3 .3 & \text { Bioavailability } & 12\end{array}$

$\begin{array}{lll}1.4 & \text { Ellagic Acid } & 13\end{array}$

$\begin{array}{lll}1.5 & \text { Anti-Cancer Mechanisms Ellagic Acid } & 15\end{array}$

$\begin{array}{lll}\text { 1.5.1 Apoptosis } & 15 \\ 1.5 .2 & 15\end{array}$

$\begin{array}{ll}\text { 1.5.2 Anti-Angiogenesis } & 17\end{array}$

CHAPTER 2. OBJECTIVES AND HYPOTHESES

$\begin{array}{lll}2.1 & \text { Overall Goals } & 18\end{array}$

2.2 Specific Hypotheses 18

2.2.1 Development of the Chitosan/Ellagic Acid Composite Films 19

2.2.2 In vitro Anti-Cancer Activity of Chitosan/Ellagic Acid Composite Films 19

2.2.3 In vivo Anti-Cancer Activity of a Chitosan/Ellagic Acid Composite Film in a Glioma Brain Cancer Model $\quad 19$

2.2.4 An Injectable Gel System for a Local Delivery of Ellagic Acid 19

CHAPTER 3. DEVEOPMENT OF CHITOSAN/ELLAGIC ACID FILMS AS A LOCAL DRUG DELIVERY SYSTEM TO INDUCE APOPTOTIC DEATH OF HUMAN MELANOMA CELLS

3.1 Summary 20

3.2 Introduction 20

3.3 Materials and Methods $\quad 22$

$\begin{array}{lll}3.3 .1 & \text { Materials } & 22\end{array}$

3.3.2 Preparation of Chitosan Films (Ch) 22

3.3.3 Preparation of Chitosan/Ellagic Acid (Ch/EA) Composite Films 22

$\begin{array}{lll}3.3 .4 & \text { FTIR Spectra } & 23\end{array}$

$\begin{array}{lll}3.3 .5 & \text { X-Ray Diffraction } & 23\end{array}$

3.3.6 Scanning Electron Microscopy 23

3.3.7 Water Contact Angle Determination 23

$\begin{array}{lll}\text { 3.3.8 Cell Culture } & 24\end{array}$

3.3.8.1 Cell Viability-Direct Contact 24

3.3.8.2 Cell Viability-Indirect Contact 24

3.3.8.3 Cell Viability-at the Interface of the Presence and Absence of the Material 25

3.3.9 Apoptosis Assay 25 
3.3.10 Statistical Analysis 25

3.4 Results 26

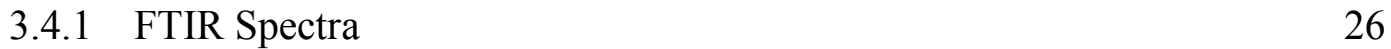

3.4.2 X-Ray Diffraction $\quad 26$

3.4.3 Scanning Electron Microscopy 26

3.4.4 Water Contact Angle Determination 30

3.4.5 Cell Viability-Direct Contact 30

3.4.6 Cell Viability-Indirect Contact 38

3.4.7 Cell Viability at the Interface of the Presence and Absence of the Material 38

3.4.8 Apoptosis Assay 38

$\begin{array}{lll}3.5 & \text { Discussion } & 41\end{array}$

3.6 Conclusion 44

CHAPTER 4. THE INHIBITION OF GLIOMA GROWTH IN VITRO AND IN VIVO BY A CHITOSAN/ELLAGIC ACID COMPOSITE BIOMATERIAL $\quad 45$

4.1 Summary 45

4.2 Introduction 45

4.3 Materials and Methods $\quad 47$

$\begin{array}{lll}\text { 4.3.1 Materials } & 47\end{array}$

4.3.2 Preparation of Chitosan Films (Ch) 47

4.3.3 Preparation of Chitosan/Ellagic Acid (Ch/EA) Composite Films 47

$\begin{array}{ll}\text { 4.3.4 In vitro Release Studies } & 47\end{array}$

4.3.5 In vitro Enzymatic Degradation $\quad 48$

$\begin{array}{lll}\text { 4.3.6 Cell Culture } & 48\end{array}$

4.3.6.1 In vitro Anti-Tumor Activity - Direct Cell Culture 49

4.3.6.2 In vitro Anti-Tumor Activity - Indirect Cell Culture 49

4.3.7 Western Blot 49

4.3.8 Caspase-3 Activation $\quad 50$

4.3.9 In vitro Angiogenesis Assay 51

4.3.10 In vivo Anti-Tumor Activity 51

4.3.11 Statistical Analysis $\quad 52$

4.4 Results $\quad 52$

4.4.1 In vitro Release Studies $\quad 52$

4.4.2 In vitro Enzymatic Degradation $\quad 54$

4.4.3 Cell Viability - Direct Contact 54

4.4.4 Cell Viability - Indirect Contact 57

4.4.5 Western Blot Analysis for p53 61

$\begin{array}{ll}\text { 4.4.6 Caspase-3 Activation } & 61\end{array}$

4.4.7 In vitro Angiogenesis Assay 61

4.4.8 In vivo Anti-Tumor Activity 65

$\begin{array}{lll}4.5 & \text { Discussion } & 71\end{array}$

$\begin{array}{ll}4.6 \text { Conclusion } & 80\end{array}$ 


\section{CHAPTER 5. DEVELOPMENT AND EVALUATION OF A CHITOSAN BASED INJECTABLE GEL FOR BRAIN CANCER TREATMENT}

5.1 Abstract

5.2 Introduction

5.3 Materials and Methods

5.3.1 Materials

5.3.2 Preparation of the Chitosan / $\beta$-Glycerophosphate Solution:

$\mathrm{Ch} / \beta$-GP

5.3.3 Preparation of the Chitosan/ $\beta$-GP Solution Loaded with Ellagic Acid: DCh/ $\beta-\mathrm{GP} / \mathrm{EA}$

5.3.4 Characterization of the Chitosan/ $\beta$-GP Gel

5.3.4.1 FTIR Spectra

5.3.4.2 Scanning Electron Microscopy

84

5.3.4.3 Rheological Analysis

85

5.3.4.4 Gelation Time Determination 85

5.3.5 In vitro Release Studies

5.3.6 In vitro Enzymatic Degradation 86

5.3.7 Cell Culture 86

5.3.7.1 Cytotoxicity of $\beta$-GP Solution on Cell Culture 86

5.3.7.2 Cytotoxicity of Chitosan/ $\beta$-GP Delivery System on Cell Culture

5.3.8 In vitro Anti-Tumor Activity

5.3.9 Statistical Analysis

5.4 Results

5.4.1 FTIR Spectra

$\begin{array}{lll}\text { 5.4.2 Scanning Electron Microscopy } & 88\end{array}$

$\begin{array}{lll}5.4 .3 & \text { Rheological Analysis } & 88\end{array}$

5.4.4 Gelation Time Determination $\quad 91$

5.4.5 In vitro Release Studies $\quad 91$

5.4.6 In vitro Enzymatic Degradation 99

5.4.7 Cytotoxicity of $\beta$-GP Solution $\quad 99$

5.4.8 Cytotoxicity of Chitosan/ $\beta-G P$ Gel 103

5.4.9 In vitro Anti-Tumor Activity of Chitosan/ $\beta-G P$ Gel 103

$\begin{array}{lll}5.5 & \text { Discussion } & 107\end{array}$

$\begin{array}{ll}5.6 \text { Conclusion } & 110\end{array}$

CHAPTER 6. DISCUSSION

6.1 Overall Goals $\quad 111$

6.2 Development of the Chitosan/Ellagic Acid Composite Films 111

6.3 In vitro Anti-Cancer Activity of Composite Films 112

6.4 In vivo Anti-Cancer Activity of Composite Films in a Glioma Model 113

6.5 Injectable Chitosan Gel Delivery System 113

$\begin{array}{ll}6.6 \text { Conclusion } & 114\end{array}$ 
CHAPTER 7. FUTURE DIRECTIONS

LIST OF REFERENCES

116

VITA

131 


\section{LIST OF TABLES}

Table 1.1 Representative local drug delivery systems and their characteristics currently in use

Table 1.2 Structural classification of biodegradable polymers used in drug delivery

Table 3.1 Water contact angles of the chitosan/ellagic acid composite films

Table 3.2 Induction of apoptosis in melanoma cells cultured on the indicated samples for 2 and 24 hours 


\section{LIST OF FIGURES}

Figure 1.1 Schematic drawings of (a) repeating unit in chitin and chitosan and (b) the enzymatic hydrolysis at glycosidic bonds of chitosan molecules

Figure 1.2 Major chemical structure of dietary phenolic substances. The two main categories are (a) non-flavonoids including gallic acid, ellagic acid and caffeic acid; (b) flavonoids including quercetin and anthocyanins

Figure 1.3 Ellagic acid and its derivatives

Figure 3.1 FTIR spectra of the chitosan/ellagic acid composite films with varying concentration of the ellagic acid

Figure 3.2 X-ray diffraction patterns

Figure 3.3 SEM micrographs on the surface of the chitosan/ellagic acid composite films with varying concentration of the ellagic acid

Figure 3.4 Photomicrographs of WM115 melanoma cells cultured on the chitosan coatings containing various concentration of ellagic acid

Figure 3.5 Photomicrographs of HS68 fibroblasts cultured on the chitosan coatings containing various concentration of ellagic acid

Figure 3.6 Viability of (a) WM115 melanomas and (b) HS68 fibroblasts via direct contact culture by MTS assay

Figure 3.7 Viability of WM115 melanomas via indirect contact culture by MTS assay

Figure 3.8 Photomicrographs of (a) WM115 melanomas and (b) HS68 fibroblasts at the boundary between the absence and presence of $\mathrm{Ch} / \mathrm{EA} 1$

Figure 4.1 In vitro cumulative amount of the ellagic acid released from the $\mathrm{Ch} /$ EA composite films

Figure 4.2 In vitro enzymatic degradation of the $\mathrm{Ch} / \mathrm{EA}$ composite films

Figure 4.3 Viability of (a) human U87 glioblastoma and (b) rat C6 glioma via direct contact culture by MTS assay 
Figure 4.4 Fluorescent images of GFP tagged human U87 glioblastoma

Figure 4.5 Fluorescent images of GFP tagged rat C6 glioma

Figure 4.6 Viability of (a) human U87 glioblastomas and (b) rat C6 gliomas via indirect contact culture by MTS assay

Figure 4.7 Western blot analysis of the p53 protein levels in the rat C6 glioma cells

Figure 4.8 Caspase-3 activity after treatment

Figure 4.9 In vitro angiogenesis assay to investigate the morphological differentiation of HUVEC into capillary-like structures

Figure 4.10 The measure of tumor volume using (a) caliper; (b) ultrasound machine; (c) fluorescence reflectance imaging (FRI) system

Figure 4.11 In vivo anti-tumor efficacy comparisons using a caliper

Figure 4.12 In vivo anti-tumor efficacy comparisons using an ultrasound machine

Figure 4.13 In vivo anti-tumor efficacy comparisons using an optical imaging system

Figure 4.14 The percentage of weight loss of animals over time periods of treatments after tumor inoculation

Figure 4.15 Histological analysis of rat C6 gliomas stained with hematoxylin and eosin

Figure 4.16 Histological analysis of rat C6 gliomas stained with hematoxylin and eosin

Figure 5.1 Photograph showing the thermal gelation of the chitosan/ $\beta$-GP solutions which were placed (a) at $5^{\circ} \mathrm{C}$ and (b) at $37^{\circ} \mathrm{C}$ for 30 minutes

Figure 5.2 FTIR spectra of the chitosan/ $\beta$-GP solution

Figure 5.3 SEM micrographs on the surface of the chitosan/ $\beta$-GP gels

Figure 5.4 The gelation temperature of the dialyzed chitosan solution

Figure 5.5 The gelation temperature of the undialyzed chitosan solution 
Figure 5.6 The gelation time of the $\mathrm{DCh} / \beta-\mathrm{GP}$ solution as a function of the temperature

Figure 5.7 In vitro cumulative amount of the ellagic acid released from the $\mathrm{DCh} / \beta-\mathrm{GP} / \mathrm{EA}$ gels in the absence of lysozyme or in the presence of lysozyme

Figure 5.8 In vitro cumulative enzymatic degradation of the $\mathrm{DCh} / \beta-\mathrm{GP}$ gels

Figure 5.9 Viability of HS68 human newborn fibroblasts via direct culture by the MTS assay

Figure 5.10 Photomicrographs showing viability of HS68 human newborn fibroblasts via the direct culture on the $\mathrm{DCh} / \beta-\mathrm{GP}$ gel

Figure 5.11 Viability of HS68 human newborn fibroblasts via indirect culture by the MTS assay

Figure 5.12 Viability of cancer cells via the indirect culture by the MTS assay 


\section{CHAPTER 1. INTRODUCTION}

Despite significant progress in anti-cancer therapy, cancer is still the second leading cause of death after heart disease and responsible for one of four deaths in the United States [ACS 2008, Jain 2001]. Approximately 1,437,180 new cases of invasive cancer in the United States are diagnosed and nearly 565,650 died of the cancer in 2008 [ACS 2008]. Especially, brain cancer is the leading cause of cancer-related death in patients younger than age 35 [ACS 2008]. Recently, local drug delivery system has been considered as a practical approach for cancer treatment compared with conventional chemotherapy. In conventional chemotherapy, cancer drugs are nonspecifically distributed, thus leading to a rapid loss of the efficacy and potential systemic toxicity into the body. In addition, for brain cancer, the blood-brain-barrier limits the access of therapeutic agents to brain tumor [Graham 2004, Wang 2002]. However, local delivery system has the potential to control drug release, thereby increasing efficacy and safety.

The common strategy of local delivery system is increasing therapeutic dose to the tumor site while reducing drug exposure to normal tissue [Groothuis 2000, Jain 2001, Moses 2003]. In particular, polymeric delivery system is implantable at a local site and biodegradable after a therapeutic span. A drug is incorporated into a polymeric device and released in a controlled manner. This delivery system can be optimized using characteristics of drugs and polymers in terms of solubility, degradation, and drug/polymer interactions.

In this regard, this section provides information of polymeric delivery systems regarding controlled drug release and degradation. In addition, ellagic acid, a naturally occurring phenolic compound, is introduced regarding its biological properties and anticancer mechanism. It is of interest to explore the feasibility of use of a dietary substance as a promising strategy for cancer treatment. Further, the study of the biological effects of chitosan/ellagic acid composite materials helps to establish a platform for a local chemotherapy.

\subsection{Local Drug Delivery Systems}

A drug delivery system is a device to maintain effective drug levels in the body for a desired time period. In the conventional delivery system, drug is frequently administered in the form of liquid or powder, causing problems associated with systemic toxicity. Local delivery system is designed for a steady release of drug at a specific site, thus remaining drug levels between maximal toxicity and minimal inefficacy [Dash 1998, Park 1996]. Current technical advances in drug delivery have enabled the drug to be incorporated into a variety of devices such as osmotic pump, transdermal patch, lipsomal encapsulation, and implantation [Dash 1998, Moses 2003]. Table 1.1 summarizes currently available local drug delivery systems and their applications. These delivery systems have been explored to optimize the therapeutic efficacy of drugs, bio-molecules, and growth factors for long term delivery regarding stability and safety. 
Table 1.1 Representative local drug delivery systems and their characteristics currently in use.

\begin{tabular}{|c|c|c|}
\hline Delivery method & Material type & Characteristics \\
\hline $\begin{array}{l}\text { External portable } \\
\text { infusion }\end{array}$ & Osmotic mini pump & $\begin{array}{l}\text { Direct intratumoral treatment } \\
\text { Continuous and controlled } \\
\text { delivery } \\
\text { Scheduled administration }\end{array}$ \\
\hline $\begin{array}{l}\text { Transdermal } \\
\text { delivery }\end{array}$ & $\begin{array}{l}\text { Adhesive \& } \\
\text { Multi-layer patch }\end{array}$ & $\begin{array}{l}\text { Diffusion through skin } \\
\text { Controlled release } \\
\text { No pains }\end{array}$ \\
\hline \multirow{2}{*}{$\begin{array}{l}\text { Intravenous infusion } \\
\text { (I.V.) }\end{array}$} & Liposomal delivery & $\begin{array}{l}\text { Prolonged drug circulation } \\
\text { (long circulation lifetime) } \\
\text { High drug capacity } \\
\text { Biodegradable and low toxicity }\end{array}$ \\
\hline & Pegylated delivery & $\begin{array}{l}\text { Prolonged drug circulation } \\
\text { Stable and non-immunogenic } \\
\text { device } \\
\text { Site specific delivery }\end{array}$ \\
\hline \multirow{3}{*}{$\begin{array}{l}\text { Polymeric } \\
\text { implantation }\end{array}$} & Micro-, nano-sphere & $\begin{array}{l}\text { Localized and sustained release } \\
\text { Biodegradable implant }\end{array}$ \\
\hline & Wafer & $\begin{array}{l}\text { Localized and sustained release } \\
\text { Biodegradable implant }\end{array}$ \\
\hline & Injectable gel & $\begin{array}{l}\text { Localized and sustained release } \\
\text { Biodegradable implant } \\
\text { Non surgical delivery }\end{array}$ \\
\hline
\end{tabular}


Osmotic pump has been employed to continuously administer a drug on a schedule and maintain a long term control of local diseases, resulting in higher therapeutic efficacy [Drixler 2000, Giussani 2003, Kisker 2001]. It is directly implanted at targeted local area, and the drug is frequently introduced with low dose and remains a sustained circulating level. ALZET mini pump has been used to avoid the blood-brain barrier (BBB) of brain and directly administer a drug to the central nervous system [Giussani 2003].

Transdermal devices have been designed for a continuously delivery of a drug through the skin into the systemic circulation in a time and dose dependent manner [Isoniemi 2001, Tonnesen 1999]. A drug is loaded in the polymeric patches and directly released into the bloodstream via difference in drug concentrations between the device and the blood flow. This system consists of liners, adherents, drug reservoirs, and drug release membrane. For instance, transdermal patches are used for patients with pulmonary disease and cardiovascular disease associated with increases in heart rate and blood pressure [Tonnesen 1999]. This system induces controlled release of the drug without pains.

Using a lipsomal encapsulation technique, therapeutic agents such as drugs, proteins, and genes are encapsulated into liposomes and circulate into the bloodstream through diffusion and liposomal degradation [Maurer 2001]. The ability to encapsulate the therapeutic agents is dependent on physicochemical properties of liposomes such as polarity, charge, and size. This system can prolong circulation time by reducing volume of drug distribution within the bloodstream and increase drug carrying capacity compared with a simple intravenous injection [Burstein 1999, Maurer 2001]. This results in reduced toxic side effects on normal tissues and increased efficacy of therapeutic agents. For example [Lyass 2000, Marina 2002], Doxil is a different form of doxorubicin which is used for cancer treatments. Doxorubicin interacts with DNA and inhibits nucleic acid synthesis. Doxil is composed of a liposome and a layer of methoxypolyethylene glycol (MPEG). MPEG is used to prevent the crosslinking and polymerization of molecules, resulting in increased biocompatibility and reducing immune response [Rose 2005]. This pegylation can protect liposomes from detection and destruction by monocytes and macrophages in the liver and spleen, resulting in extended therapeutic span [Maurer 2001].

The major problems in the conventional chemotherapy are attributed to an initial burst effect of drugs, leading to a rapid loss of the efficacy and harmful side effects into the body. In this regard, implantable polymeric delivery system has gained a lot of attention for controlled steady release, thus extending therapeutic span and minimizing side effects [Dash 1998, Park 1996]. Drugs are physically embedded or chemically conjugated in polymeric devices such as gels, beads, or wafers.

For instance, gliadel ${ }^{\circledR}$ wafer has been used to deliver carmustine (BCNU) into the surgical cavity for brain cancer treatment [Olivi 2003, Perry 2005]. It consists of carboxyphenoxypropane (CPP) and sebacic acid (SA) in the polymer. The carmustine is distributed into the copolymer and functions as an alkylating agent, which treats brain 
cancer including glioma and glioblastoma multiforme [Brem 2001]. When the anhydride bonds in the copolymer degrade, carmustine is released from the gliadel wafer and diffuses into the adjacent brain tissue. During the biodegradation of the gliadel wafer, carboxyphenoxypropane is eliminated by the kidney while sebacic acid is metabolized by the liver [Brem 2001, Hanes 1996, Katti 2002].

As an example of implantable devices, poly (lactic acid-co-glycolic acid) (PLGA) microspheres have been studied to deliver 5-fluorouracil (5-FU), which is a hydrophilic and antimetabolite drug used for cancer treatment [Menei 1999, 2004]. After surgical resection of the tumor, 5-fluorouracil (5-FU) loaded PLGA microspheres are implanted intracranially to extend therapeutic duration and to avoid systemic toxicity around brain tissue. The implantable microspheres have been shown to be biocompatible and biodegradable.

\subsection{Implantable Polymeric Delivery System}

One of major considerations in development of local delivery systems is biocompatibility. Polymeric systems are implantable and sustained contact with the local tissues, thus, polymers and degradable products should be non-toxic. They may be synthetic or natural, and biodegradable or non-biodegradable. In particular, the biodegradable delivery systems alleviate the need for surgical removal of the implant, thereby increasing patient acceptance and compliance [Gunatillake 2003]. They are disintegrated by hydrolysis of the polymer chains into biologically acceptable polymer products over time. The biodegradable polymers have hydrolysable linkages such as ester, amide, anhydride, orthoester, urea, carbonate, and urethane in their backbone [Gunatillake 2003]. They are absorbed or excreted from the body via normal metabolic pathways without any complications. The biodegradation of the polymers is attributed to some factors such as polymer structure, composition, morphology, surface structure, molecular weight, shape, size, and etc [Dash 1998, Jain 2000]. Drug release of biodegradable systems is governed by diffusion, initial drug loading, drug/polymer interactions, drug solubility, and polymer degradation [Gunatillake 2003]. In contrast, non-biodegradable polymeric systems need a minor surgery to remove the implant. The drug release is achieved by diffusion via pores in the polymer matrix or between polymer chains [Dash 1998].

\subsubsection{Biodegradable Polymers}

Biodegradable polymers such as polyesters, polyamides, polyanhydrides, polyorthoesters, polycarbonates and polyurethanes have been most extensively studied for controlled drug release. Table 1.2 summarizes the structure of key biodegradable polymers used in drug delivery systems. Among biodegradable polyesters, poly(lactic acid) (PLA), poly(glycolic acid) (PGA), or their copolymers poly(lactic-co-glycolic acid) (PLGA) have been extensively studied in the forms of film, micro or nanosphere, and hydrogel [Fialho 2005, Jeong 2000, Yamaguchi 2002]. 
Table 1.2 Structural classification of biodegradable polymers used in drug delivery.

\begin{tabular}{ll}
\hline Polymer class & Structure of repeating unit \\
\hline Polyesters & \\
Polyanhydrides & \\
Polyorthoesters & \\
Polycarbonates & \\
\cline { 2 - 2 } & \\
\hline
\end{tabular}


Drugs are released by the cleavage of the polymeric chains via hydrolytic degradation or enzymatic degradation while polymers breakdown into their monomers and metabolized under physiological conditions [Gunatillake 2003]. In the copolymers (PLGA), the molecular weight and the ratio of PLA and PGA monomers play an important role in controlling the drug release [Jain 2000]. The lower molecular weight polymer has, the faster it degrades, and copolymer degrades faster than the homopolymers.

Polyanhydrides degrade fast in an aqueous environment by hydrolysis of anhydride linkages. They have unique degradation properties for the controlled drug release, which is mostly affected by their surface degradation [Gopferich 2002]. The most well studied polyanhydrides, for example, is poly [1,3-bis (carboxyphenoxy) propane-cosebacic-acid] p(CPP-SA) used for patients with brain tumors [Dang 1996, Katti 2002]. Unlike poly (lactic-co-glycolic acid) (PLGA), which is degraded by bulk erosion, polyanhydride is degraded by surface erosion. The surface erosion occurs only at the surface of the polymer while the bulk erosion includes entire polymers. The physical properties of $\mathrm{p}(\mathrm{CPP}-\mathrm{SA})$ can be altered by different ratio of monomers (CPP:SA), influencing polymer degradation rate thereby controlling drug release behavior [Dang 1996, Gopferich 2002]. The polyanhydride polymers provide several advantages such as constant release (zero order kinetics) over a long period of time, controllable release rate, and degradation after therapeutic span [Katti 2002].

Polyorthoesters are also biodegradable polymers by surface erosion and fabricated into wafers, microspheres, injectable semi-solid materials. The polymer erosion occurs by hydrolysis mostly in the outer layers of the polymer matrix, and the drug is released concomitantly [Heller 2002]. The drug release rate is proportional to initial drug loading rate. The local acidity attributed to polymer degradation can be controlled and a neutral $\mathrm{pH}$ inside polymeric device remains because hydrolysis products generally diffuse away from the device [Heller 2000, Zignani 2000]. In others, tyrosinederived polycarbonates and polyutethanes have been investigated as biodegradable polymers [Tangpasuthadol 2000]. Tyrosine-derived polycarbonates contain three hydrolysable bonds such as amide, ester, and carbonate and yield alcohols and carbon dioxide after hydrolysis of the carbonate groups. Polyurethanes have been used in a broad range of biomedical applications due to their good mechanical properties and biocompatibility [Simmons 2008]. However, the toxicity generated from degradation products should be considered.

There are many types of non-degradable polymers. They have been studied for controlled drug release applications, including poly [ethylene-co-(vinyl acetate)] (EVAc), poly(methyl methacrylate) (PMMA), poly(ethylene glycol) (PEG), Poly ( $\mathrm{N}$-isopropyl acrylamide) (PNIPAAm), and poly(dimethylsiloxane) (PDMS) [Liu 2005, Park 1996, Zhang 2003]. Slow diffusion via the polymeric delivery system provides sustained drug release from the delivery system [Dash 1998]. However, a major disadvantage of these delivery systems is a need for surgical removal of the implants after the completion of therapy. In order to approach effective sustained release from, the polymer networks, interactions between drugs and polymer molecules are also critical. In this regard, non- 
biodegradable polymers are not preferred for large molecular weight drugs and biomolecules, and they may induce any risks related to inflammation and long term complications.

\subsubsection{Natural Chitosan Polymer}

Natural polymers such as polysaccharides, collagen, albumin, and cellulose derivatives are also employed for drug delivery because they are non-toxic, biocompatible, and biodegradable. Most natural polymers are degraded by enzymatic hydrolysis. Recently, chitosan has been of great interest among polysaccharides due to the presence of nitrogen group, giving rise to chemical reactions with other chemicals. It has been studied for a variety of biomedical applications, including wound healing, tissue regeneration, and drug/gene delivery [Kumar 2000, Pillai 2009].

Chitosan is the fully or partially N-deacetylated derivative of chitin, which is the most abundant natural amino polysaccharide, obtained from shellfish sources such as crab and shrimp [Kumar 2000, Pillai 2009]. Chitosan and chitin have structural similarity to cellulose in plants, and they are copolymers consisting of $\mathrm{N}$-acetyl-glucosamine and $\mathrm{N}$ glucosamine units [Chatelet 2001]. Chitin contains higher than $50 \%$ of $\mathrm{N}$-acetylglucosamine units and has limited solubility in common solvents and chemically less reactive (Figure 1.1a). In contrast, chitosan contains higher proportion of $\mathrm{N}$-glucosamine units (above $50 \%$ ) in the polymer than chitin. It is soluble in dilute acids and has better reactivity for chemical reactions [Khan 2002].

Unlike the other polysaccharides such as cellulose and dextran, chitosan is a positively charged polysaccharide in acidic solvent along with its molecules. Amine groups $\left(\mathrm{NH}_{2}\right)$ of chitosan are protonated $\left(\mathrm{NH}_{3}{ }^{+}\right)$in an acidic solution, presenting an important characteristic of chitosan. Several parameters responsible for free amine groups of chitosan include the degree of deacetylation (DDA), molecular weight, and environmental factors such as $\mathrm{pH}$, solvent, temperature, and etc [Wang 2006]. Increase in the degree of deacetylation (DDA) significantly lowers the protonation constants $\mathrm{pKa}$, influencing hydrophobic interactions and hydrogen bondings between chitosan molecules [Wang 2006]. Increase in molecular weight of chitosan also lowers the protonation constants $\mathrm{pKa}$.

Chitosan is hydrolyzed by different enzymes such as chitosanase and lysozyme [Muzzarelli 1997, Pangburn 1982, Tomihata 1997]. The chitosanase is completely absent in mammals but lysozyme is mainly responsible for the degradation of chitosan in human body. Lysozyme is present in tissues, saliva, blood, and tears, and it is used by phagocytic cells during the inflammatory response [Pillai 2009]. Lysozyme hydrolyses glycosidic bonds of chitosan molecules, but it is less active on chitosans with higher DDA [Pangburn 1982, Stokke 1995].

The hydrolysis reaction of the lysozyme includes proton $\left(\mathrm{H}^{+}\right)$transfer from a carboxyl group of a glutamic acid (Glu-35) to the substrate and then a hydroxyl group of 


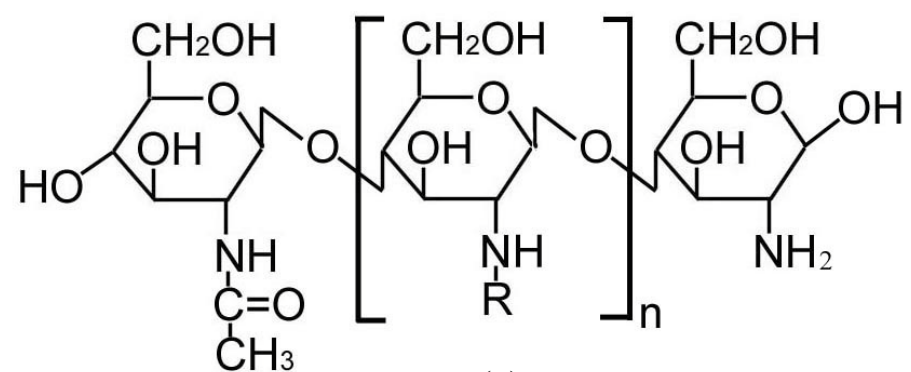

(a)

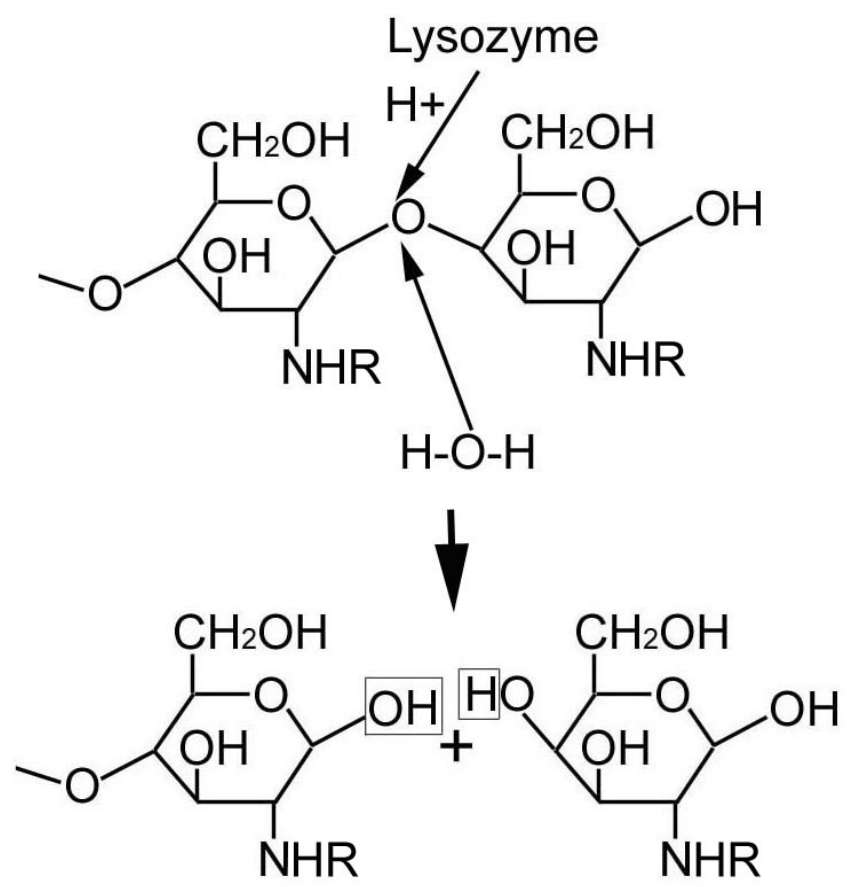

(b)

Figure 1.1 Schematic drawings of (a) repeating unit in chitin and chitosan and (b) the enzymatic hydrolysis at glycosidic bonds of chitosan molecules. $\mathrm{R}$ is acetyl $\left(\mathrm{COCH}_{3}\right)$ or hydrogen $(\mathrm{H})$ group. Chitin has more than $50 \%$ of acetyl groups while chitosan has more than $50 \%$ of hydrogen groups in their backbone. Lysozyme attacks the glycosidic bond by donating a proton and a water molecule reacts with a carbon atom in an amino sugar group, leading to hydrolysis.

Sources:

Muzzarelli R. Human enzymatic activities related to the therapeutic administration of chitin derivatives. Cellular and Molecular Life Sciences. 1997;53:131-140.

Pillai CKS, Paul W, Sharma CP. Chitin and chitosan polymers: Chemistry, solubility and fiber formation. Progress in Polymer Science 2009;34:641-678. 
a water molecule is added to a carbon atom in an amino sugar group [Muzzarelli 1997]. As shown in Figure 1.1b, chitosan is biodegradable into amino sugar groups via the metabolic pathways of glycosaminoglycans and glycoproteins [Chatelet 2001]. Degradation rate of chitosan polymer can be controlled using the degree of deacetylation (DDA) and molecular weight which are considered as critical parameters influencing physicochemical properties of chitosan [Li 2007, Wang 2006].

Chitosan has beneficial properties such as biocompatibility, biodegradability, non-toxicity, low immunogenicity, anti-bacterial, anti-fungal, and anti-viral activity [Kumar 2000]. Recently, chitosan is considered as a promising biodegradable carrier for controlled drug release with respect to safety and efficacy of drug therapy [Ravi- Kumar 2006]. Its positively charged molecules interact with negatively charged therapeutic agents and biomolecules. Generally, drugs are chemically attached or dispersed into the

chitosan matrix and released via slow and controllable diffusion or biodegradation [Freier 2005, Hong 2007, Ren 2005].Chitosan has been fabricated in a variety of form such as film, sponge, hydrogel, and beads. Chitosan itself has an ability to form a gel and can be modified via its amino and hydroxyl groups [Wang 2006]. Chitosan has been incorporated with the other polymers or crosslinking reagents via chemical modification. Chemical modifications of chitosan have been studied to improve its solubility and extend applications via etherification, esterification, cross-linking, and graft copolymerization [Gorochovceva 2004, Jayakumar 2005, Sashiwa 2004, Yoshifuji 2006]. These modifications influence physicochemical and biological properties of chitosan as a carrier for various therapeutic agents.

\subsection{Dietary Polyphenols}

The recent interest in the biological activity of the phenolic compounds is a result of the potential health benefits of dietary constituents. It is well known that consumption of polyphenol-rich foods or beverages can prevent various human diseases. The polyphenols obtained from fruits, vegetables, and herbs are protective against cardiovascular disease, inflammation and cancer [Stoclet 2004]. The emerging evidence showed their drug-like effects on human body in many ways, but low side effects compared with pharmaceutical drugs. Health benefits of several polyphenols have been examined in their extract forms [Scalbert 2000]. The chemical structure of polyphenols influences bioavailability, antioxidant activity, and interactions with cells or enzymes [Han 2007, Scalbert 2000, Williamson 2000]. This review summarizes the current understanding of the biological activity of polyphenols and their feasibility for a local drug delivery.

\subsubsection{Chemical Structure}

The dietary phenolic compounds contain the most abundant antioxidants in human diets, contributing to anti-carcinogenic or cardio-protective action [Han 2007, Stoclet 2004]. The phenolic compounds, plant secondary metabolites, contain a number 
of phenolic hydroxyl groups, inducing the antioxidant activity [Rice-Evans 1996]. They can be subdivided into several groups according to their structural differences. More than 8,000 different phenolic structures have been studied and broadly categorized into 10 classes [Scalbert 2000]. But, only a limited number of phenolic substances have been used for human diets.

The two main categories are non-flavonoids (e.g. phenolic acids, stilbenes) and flavonoids as shown in Figure 1.2 [Scalbert 2000]. The phenolic compounds are often attached to sugars (glycosides) and most of them are water soluble. The phenolic acids have simple molecular structures derived from hydroxybenzoic acids such as ellagic acid, caffeic acid, gallic acid, and etc [Stoclet 2004]. The flavonoids are the most abundant and account for two thirds of the total phenolic compounds [Scalbert 2000]. They can be divided into several classes, including flavonols, flavones, isoflavones, anthocyanins, flavanols, and etc [Han 2007]. Basically, they have two phenols connected by a pyran carbon ring structure. Individual differences of phenolic substances are attributed to the variation of hydroxyl groups, oxygens, sugars, and methyl groups [Rice-Evans 1996].

These structural diversities occur via hydroxylation of the phenolic rings, glycosylation, and acylation [Scalbert 2000, Han 2007]. The structural diversity influences absorption, distribution, bioavailability, and biological properties of phenolic compounds. The antioxidant activity is dependent on the relative positions of the hydroxyl groups in the ring structures [Rice-Evans 1996].

\subsubsection{Biological Properties}

A wide range of biological properties of the phenolic compounds have been extensively reviewed. Many scientists have investigated the importance of the use of fruits and vegetables for health care to verify their therapeutic effects. Many studies demonstrated the phenolic compounds have anti-carcinogenic, anti-inflammatory, antibacterial, anti-allergic, and anti-viral properties [Han 2007, Loo 2003, Scalbert 2000]. In addition, the dietary phenolic products show the protective effect against cardiovascular risk by inhibiting the angiogenic process and preventing the growth of endothelial and vascular smooth muscle cells [Stoclet 2004].

The main action of phenolic compounds is direct control of the oxidation stress by modulating the free radicals in biological systems [Loo 2003, Rahman 2006]. Oxidation stress may occur when antioxidant defense systems fail to manage the production of free radicals. The imbalance between antioxidants and free radicals results in oxidative stress, leading to cellular damage. The free radicals are highly reactive and a main cause of accelerated aging, degenerative diseases, and abnormal cell growth [Han 2007]. Many dietary phenolic substances have been investigated to reduce the level of free radical via a number of potential antioxidative mechanisms. They have been studied concerning antioxidant capacity and regulation of cellular activity. The phenolic compounds can act as antioxidants by breaking the free radical chain reaction [Scalbert 2000]. Antioxidants protect biological system against the reactive oxygen species (ROS) 


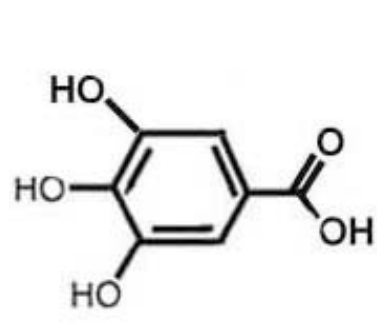

Gallic acid

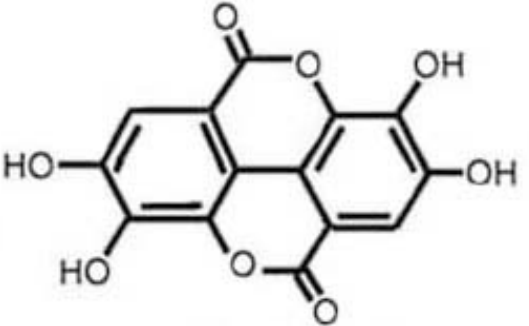

Ellagic acid<smiles>O=C(O)/C=C/c1ccc(O)c(O)c1</smiles>

Caffeic acid

(a)<smiles>O=c1c(O)c(-c2ccc(O)c(O)c2)oc2cc(O)cc(O)c12</smiles>

Quercetin<smiles>Oc1cc(O)c2ccc(-c3cc(O)c(O)c(O)c3)[o+]c2c1</smiles>

Anthocyanins

(b)

Figure 1.2 Major chemical structures of dietary phenolic substances. The two main categories are (a) non-flavonoids including gallic acid, ellagic acid and caffeic acid; (b) flavonoids including quercetin and anthocyanins. The phenolic substances vary structurally with substituent groups and linkages. Main structural difference is presence of a pyran carbon ring structure which connects two phenols in flavonoids.

Sources:

Aggarwal BB, Shishodia S. Molecular targets of dietary agents for prevention and therapy of cancer. Biochemical Pharmacology 2006;71:1397-1421.

Han X, Shen T, Lou H. Dietary polyphenols and their biological significance. International Journal of Molecular Sciences 2007;8:950-988.

Scalbert A, Williamson G. Dietary intake and bioavailability of polyphenols. The Journal of Nutrition 2000;130:2073S-2085S. 
such as oxygen, superoxide, peroxyl radicals, and hydroxyl radicals [Loo 2003, Rahman 2006].

The antioxidant activities of the phenolic compounds depend on their ability to scavenge free radicals and chelate metal ions [Loo 2003, Rahman 2006, Scalbert 2000]. The molecular structure of phenolic compounds highly influences antioxidant capacity. The hydroxyl groups associated with phenolic rings play an important role in scavenging free electrons, resulting in decease in oxidative reactions of biological molecules [Loo 2003, Rice-Evans 1996]. Phenolic compounds chelate the metal ions such as copper or iron in the oxidation systems [Rice-Evans 1996]. Such chelation of catalytic metal ions can prevent the formation of the reactive hydroxyl radicals. Therefore, biological activities of the phenolic compounds correspond to specific characteristics including metal reducing potential, chelating behavior, $\mathrm{pH}$, bioavailability, and solubility [Rahman 2006, Rice-Evans 1996, Scalbert 2000]. However, the relationship between biological activities of the phenolic compounds and their antioxidant properties needs further study.

The phenolic compounds also take part in modulating the activation of protein, enzymes and transcription factors in the metabolic pathways. Several studies demonstrated that phenolic compounds have structural similarities with biological molecules in size, molecular weight, and partial hydrophobicity [Han 2007, Loo 2003]. In this regard, phenolic compounds have an ability to inhibit the development of degenerative diseases and limit the activation of carcinogens.

\subsubsection{Bioavailability}

There is limited bioavailability of the phenolic compounds due to the poor absorption in the body and high metabolism in human [Rahman 2006, Scalbert 2000]. The bioavailability of phenolic compounds has been investigated by measuring their concentrations in plasma and urine after the ingestion [Scalbert 2000]. Some studies have demonstrated that the absorption of the phenolic compounds after the ingestion depends on the amounts consumed and their bioavailability [Scalbert 2000]. Most phenolic compounds containing glycosides and ester groups are hydrolyzed to the free aglycone by intestinal enzymes or colonic microflora before they are absorbed [Manach 2003, Spencer 1999, Scalbert 2000]. During a process of absorption, phenolic compounds are conjugated by methylation, sulfation, and glucuronidation in the small intestine and liver [Rice-Evans 1996, Spencer 1999]. This process is controlled by distribution of specific enzymes involved in phenolic metabolism. The conjugation mechanisms are highly effective to reduce concentration of aglycones in blood after consumption of dietary phenolic compound [Stoclet 2004]. Unlike enzymes in human tissues, colonic microflora produces more simple phenolic compounds, such as phenolic acids [Manach 2003, Scalbert 2000].

The chemical structure of phenolic compounds is an important factor contributing to the bioavailability. It influences the rate and extent of intestinal absorption and metabolites in the plasma [Manach 2003, Spencer 1999]. Some phenolic compounds, 
such as flavonoids including flavonols, isoflavones, flavones and anthocyanins, are glycosylated [Spencer 1999]. This glycosylation influences chemical, physical and biological properties of the phenolic compound. The majority of sugars connected to phenolic rings are glucose or rhamnose, and their possible positions are various. The sugars are removed by glycosidases in the first step of phenolic metabolism [Day 2001]. The molecular weight also affects the absorption rate of phenolic compounds. Proanthocyanidins are the least well absorbable phenolic compound in the small intestine due to their large molecular weight [Scalbert 2000]. The hydrophilicity of glycosylated phenols influences passive diffusion across the small intestine [Rice-Evans 1996]. The dosage difference also affects the main site of metabolism. High dosages are metabolized mostly in the liver while low dosages are metabolized by the intestinal mucosa [Manach 2003, Spencer 1999]. In addition, phenolic compounds can penetrate tissues but they are less accumulative on specific target tissue [Spencer 1999]. Many studies have focused on verifying biological properties of phenolic compounds contributing to human health. However, there often exist inconsistent results between the studies of the in vitro and in vivo, mainly due to the limited bioavailability of phenolic compounds [Han 2007].

\subsection{Ellagic Acid}

Ellagic acid is one of extensively studied phenolic compounds. It presents in various fruits and nuts such as raspberries, strawberries, blackberries, grapes, pomegranates, walnuts, pecans, and barks [Mullen 2003, Vekiari 2008]. The high therapeutic levels of ellagic acid have been obtained from red raspberries, strawberries, and pomegranates compared to other sources [Pinto 2008]. In recent years, increasing evidence has shown that the ellagic acid has the antioxidant and anti-cancer properties against cancer [Pinto 2008, Vekiari 2008]. It is safe, low-priced, and supplied as a dietary food. Ellagic acid has an ability to induce apoptotic cell death of cancers in cervical, skin, breast, lung, esophageal, brain, colon, liver, and etc [Edderkaoui 2008, Losso 2004, Narayanan 1999, Labrecque 2005]. It also inhibits formation and growth of cancer cells by influencing cell cycle arrest and preventing destruction of the p53 gene by cancer cells [Dixit 1986, Edderkaoui 2008, Khan 2007, Mertens-Talcott 2003, 2005]. It functions as a scavenger by binding cancer-causing chemicals and inactivating them [Barch 1996, Scalbert 2000]. Some researches indicated that ellagic acid also contains antiinflammatory, anti-bacterial, and anti-viral properties [Losso 2004, Whitley 2003].

Ellagic acid itself is not naturally present in plants, but found in the form of ellagic acid glycosides or hydrolyzable ellagitannins as shown in Figure 1.3 [Feldman 2005, Mullen 2003]. The ellagic acid glycosides are various according to their chemical structures. Their common structures include a single sugar group joined to a hydroxyl group at 4 position of ellagic acid. Ellagitannins contain one or more hexahydroxydiphenoyl groups (HHDP) which are conjugated to sugar groups or glucose groups [Feldman 2005]. Ellagitannins are easily converted into the ellagic acid via hexahydroxydiphenoyl groups (HHDP) after breaking ester linkages between glucose and ellagic acid by hydrolysis. The types of ellagitannins are various according to the number of HHDP groups, the location of ester groups, and the conformation of the glucose ring 

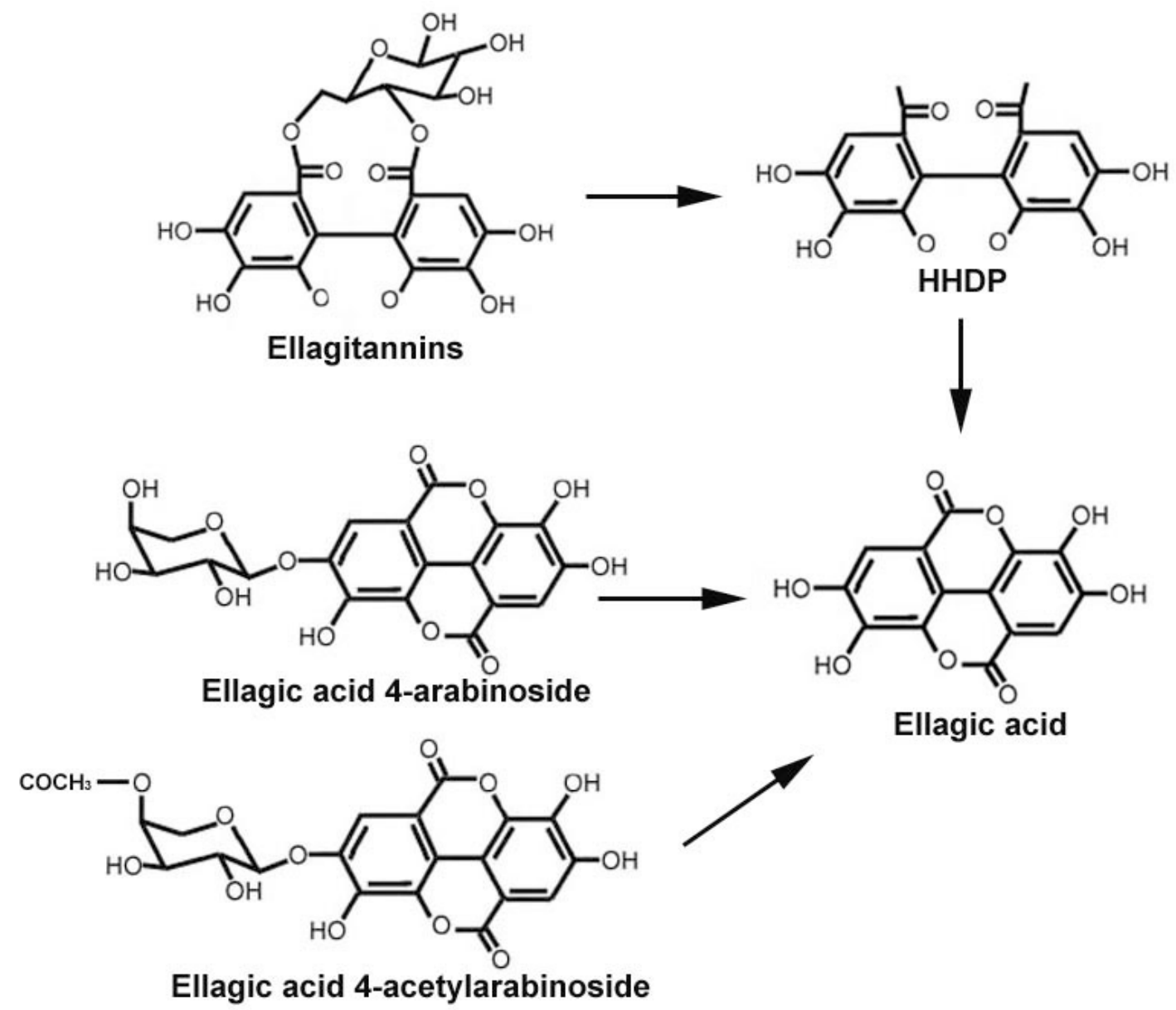

Figure 1.3 Ellagic acid and its derivatives. Ellagitannins are hydrolyzed to hexahydroxydiphenoyl groups (HHDP) which are spontaneously converted to ellagic acid. Different ellagic acid glycosides are hydrolyzed to ellagic acid.

Sources:

Feldman KS. Recent progress in ellagitannin chemistry. Phytochemistry $2005 ; 66$ :19842000.

Mullen W, Yokota T, Lean ME, Crozier A. Analysis of ellagitannins and conjugates of ellagic acid and quercetin in raspberry fruits by LC-MSn. Phytochemistry. 2003;64:617624. 
[Mullen 2003, Vekiari 2008]. Ellagic acid is generally produced in a highly concentrated form from seeds. Its seeds are processed into the flour with regard to maximum bioavailability and convertability into ellagic acid [Vekiari 2008].

Ellagic acid is a complex molecule containing four hydroxyl groups and two lactone groups. It has been suggested that different portions of the ellagic acid such as 3hydroxyl and 4-hydroxyl groups influence different anti-cancer activities [Barch 1996, Feldman 2005]. Lactone groups of ellagic acid are also known to be essential to induce anti-cancer activity [Barch 1996]. One ellagic acid molecule looks like a combination of two gallic acid molecules. Thus, the carboxylic acid joined with a phenolic ring of gallic acid possibly functions as the lactone groups in ellagic acid [Barch 1996]. In this regard, during the processing, the structural change of ellagic acid may significantly influence its solubility and bioavailability as well as anti-carcinogenic activities [Dixit 1986, RiceEvans 1996]. Studies of bioavailability showed that ellagic acid was found in the stomach and from the action of the gut microflora after consumption [Scalbert 2000, Seeram 2004, 2006]. However, ellagic acid is poorly absorbed and present in low concentrations, leading to the limited bioavailability [Scalbert 2000, Seeram 2006].

\subsection{Anti-Cancer Mechanisms}

\subsubsection{Apoptosis}

Apoptosis is a form of programmed cell death and a highly regulated process to remove damaged cells [Bai 2006]. Apoptosis represents a set of morphologic changes, including chromatin condensation, nuclear fragmentation, membrane blebbing and cell shrinkage [Khan 2007]. Unlike necrosis causing inflammatory response to surrounding tissues, apoptosis maintains the contents of the cell within the cell membrane when it is degraded. Subsequently, the apoptotic cells are phagocytosed by macrophages without inducing inflammation [Bai 2006]. Induction of apoptosis is a preferential target for cancer chemotherapy. Many studies have shown that dietary substances have an ability to induce apoptosis of cancer cells [Ahmad 2001, Gopalakrishna 2002, Lazze 2004, Loo 2003, Losso 2004, Mertens-Talcott 2003, 2005, Rahman 2006, Schuler 2000].

Numerous studies have suggested that the phenolic compounds inhibit the proliferation of cancer cells by inducing cell cycle arrest or apoptosis. This is mainly because of its ability to scavenge reactive oxygen species (ROS) such as hydrogen peroxide $\left(\mathrm{H}_{2} \mathrm{O}_{2}\right)$ [Loo 2003, Rahman 2006, Sun 1996]. $\mathrm{H}_{2} \mathrm{O}_{2}$ is a destructive toxic molecule and easily converted to hydroxyl radicals $(\mathrm{HO})$ which cause oxidative damages. It also acts as an important signaling molecule in the mitogen-activated protein kinase (MAPKs) pathways [Loo 2003, Rahman 2006, Sun 1996]. The MAPK cascades include extracellular signal-related protein kinases (ERKs), c-Jun N-terminal kinases (JNKs), and p38 kinases [Loo 2003]. The ERK pathway is activated by growth factors and promotes cell growth and survival. The JNK and p38 kinases are activated in response to oxidative stress and involved in apoptosis. MAPKs activate redox-sensitive transcription factors 
such as nuclear factor-kappa B (NF- $k \mathrm{~B}$ ) and activator protein-1 (AP-1), promoting the survival and proliferation of cancer cells [Edderkaoui 2008, Lee 2003, Loo 2003, Mertens-Talcott 2005, Rahman 2006, Sun 1996]. In this manner, the phenolic compounds can induce apoptosis by scavenging the excessive free radicals, thereby blocking MAPK signaling pathway.

The phenolic compounds can also induce apoptotic process via the intrinsic mitochondrial pathway [Khan 2007]. The intrinsic apoptotic pathway involves the expression of regulatory proteins such as increased Bax, the release of cytochrome c, and increased caspase activity, as well as decreased Bcl-2 and Bcl- $\mathrm{X}_{\mathrm{L}}$ [Larrosa 2006, Lee 2003, Schuler 2000]. The actions of pro-apoptotic and anti-apoptotic proteins are frequently associated with the activation of p53. The transcription factor p53 is an important key protein known as a tumor suppressor, regulating cell cycle and apoptosis. In cancer cells, the function of p53 can be lost by various mechanisms such as mutations [Bai 2006]. In normal cells, the p53 is usually inactive and sustained at low level by a continuous degradation. It is bound to the protein MDM2, which prevents the function of p53 and induces the degradation by transporting from the nucleus to the cytosol [Aggarwal 2006, Bai 2006]. In response to DNA damages, the p53 is activated by phosphorylation at the N-terminal region and accumulated in the nucleus [Bai 2006]. The representative target genes of $\mathrm{p} 53$ are $\mathrm{p} 21^{\text {Waf1/Cip1 }}$, growth arrest and DNA-damage inducible protein 45 (Gadd45) and the Bcl-2 family[Ahmad 2001, Bai 2006, Khan 2007]. They are mostly involved in cellular responses such as cell cycle arrest, DNA repair, apoptosis and senescence.

The intrinsic pathway is triggered by the signal factors from mitochondria in response to cellular stresses such as DNA damage, heat shock, hypoxia, and oncogene overexpression [Aggarwal 2006, Bai 2006]. The pro-apoptotic Bcl-2 family proteins are involved in this pathway and induce caspase activity from the mitochondria. The proapoptotic proteins such as PUMA, NOXA, and BID attach to the outer mitochondrial membrane for the release of the internal content [Aggarwal 2006, Khan 2007]. In response to apoptotic signaling, BID activates BAX and inserts it into the outer mitochondrial membrane. The activated BAX forms an oligomeric pore, releasing cytochrome $\mathrm{c}$ and other pro-apoptotic factors from the mitochondria. BAK is another proapoptotic protein inside the mitochondria, also induces the release of cytochrome c, leading to the activation of caspases. However, the anti-apoptotic Bcl-2 proteins such as Bcl-2 itself and BCL-X $\mathrm{L}_{\mathrm{L}}$, also bind to BID and inhibit the activation of BAX or BAK [Bai 2006, Khan 2007]. After being released from the mitochondria, cytochrome c makes a complex structure with adenosine triphosphate (ATP) and adaptor apoptotic protease activating factor-1 (Apaf-1) in the cytoplasm [Bai 2006]. This complex activates caspase9, in turn, the activated caspase-9 forms a large multi-protein structure known as the apoptosome in combination with the cytochrome c, ATP and Apaf-1. As a result, caspase3 , 6, and 7 are activated and initiate the degradation, leading to apoptosis [Bai 2006, Khan 2007].

In contrast, the extrinsic pathway is initiated outside the cell through the transmembrane death receptors such as the Fas receptors on the cell membrane 
[Ashkenazi 1998, Khan 2007]. The signal molecules released from other cells bind to transmembrane death receptors on the target cell to induce apoptosis. The extrinsic pathway initiates a signaling cascade and induces the activation of the caspase system independently of the p53 protein. However, both the pathways induce caspase- 3 activation.

\subsubsection{Anti-Angiogenesis}

Angiogenesis represents a process of a new vessel formation from pre-existing endothelial vessels. The angiogenesis is an important process for wound healing as well as tumor growth and metastasis [Oak 2005, Rundhaug 2003]. It is different from the vasculogenesis, forming new blood vessels in the absence of existing blood vessels. Angiogenesis involves proteolysis of the extracellular matrix, proliferation and migration of endothelial cells, as well as synthesis of new matrix components [Rundhaug 2003, Stoclet 2004]. The matrix metalloproteinases (MMPs) play an important role in degradation of the surrounding extracellular matrix and basement membrane. They allow endothelial cells to detach and migrate into a new tissue during angiogenesis. They are also able to release pro-angiogenic factors such as bFGF, VEGF, and TGF $\beta$ [Oak 2005, Rundhaug 2003].

Recent studies have showed ellagic acid chelates divalent cations such as $\mathrm{Zn}^{2+}$ and $\mathrm{Cu}^{2+}$, inactivating MMPs, thus inducing anti-angiogenesis [Labrecque 2005, Loo 2003, Oak 2005, Stoclet 2004]. Chelation of copper ions affects the function of angiogenic growth factors, thus inhibiting the angiogenic process in tumor cells [Aggarwal 2006, Oak 2005]. As a result of the action of ellagic acid, tumor growth and formation of new blood vessels can be suppressed by lowering level of the copper supply. In this regard, controlling the actions of the cellular and molecular levels via antiangiogenic mechanism will provide a novel approach for cancer treatment. 


\section{CHAPTER 2. OBJECTIVES AND HYPOTHESES}

\subsection{Overall Goals}

The overall objective of this project was to develop an implantable polymeric system as a novel drug delivery approach for a local chemotherapy. A variety of implantable polymeric devices have been applied for controlled drug release. In this project, chitosan, a cationic natural polymer, was employed as a drug carrier for sustained drug release, biocompatibility and biodegradation. Ellagic acid (EA), a naturally occurring phenolic compound, was incorporated into the chitosan polymer network to provide anti-cancer activity at a local targeted site.

The rationale for this project relates to sustained delivery of ellagic acid for a cancer treatment. Ellagic acid has been supplied as a dietary source to reduce the risk of cancer. However, such dietary phenolic compounds have the limited bioavailability because of their poor absorption and high metabolism in human body. In an attempt to maximize therapeutic efficacy and safety, the studies performed herein present implantable chitosan based drug carriers for local delivery of ellagic acid and their biological activities. The combination of the chitosan with the ellagic acid suggests a novel drug delivery to avoid a rapid loss of efficacy and significant toxicity into body. Chitosan/ellagic acid delivery systems were fabricated and investigated regarding localized effect of ellagic acid against cancer cells in vitro and in vivo.

Chapter 3 in this project gives a description of development and characterization of chitosan/ellagic acid composite films and presents their anti-cancer activities against a human melanoma skin cancer. This section discusses the feasibility of the use of ellagic acid in a chitosan film and localized effect of ellagic acid for a cancer treatment. Chapter 4 discusses the potential mechanisms by which the chitosan/ellagic acid composite materials induce anti-proliferative effect against malignant glioma cells. This section also presents an animal model for brain glioma study focusing efficacy and safety of local treatment in vivo. Chapter 5 gives a description of a chitosan based thermo-sensitive gel for a local delivery of ellagic acid using a simple injection. This section emphasizes the mechanism of the sol-gel transition and discusses the biocompatibility of the delivery system as well as the anti-tumor activity of ellagic acid. While the first two sections present a surgically implantable delivery system with therapeutic efficacy, the third section focuses on developing injectable thermo-sensitive gel. The motivation for the selection of the injectable delivery relates to clinical application for a non-surgical implantation to avoid additional costs and surgical pains. Chapter 6 summarizes and discusses findings of this project. Finally, chapter 7 suggests future works for this study.

\subsection{Specific Hypotheses}

Overall hypothesis is that chitosan/ellagic acid composite materials induce anticancer activity in local targeted sites by inducing apoptotic death and suppressing 
angiogenesis. In order to test the overall hypothesis, eight specific hypotheses are formulated as follows:

\subsubsection{Development of the Chitosan/Ellagic Acid Composite Films}

Hypothesis 1: Ellagic acid is chemically conjugated and embedded into chitosan polymer for a local delivery.

Hypothesis 2: Chitosan/ellagic acid composite films biodegrade and provide a sustained slow release of ellagic acid at targeted areas.

\subsubsection{In vitro Anti-Cancer Activity of Chitosan/Ellagic Acid Composite Films}

Hypothesis 3: Anti-cancer activity of chitosan/ellagic acid composite films is attributed to ellagic acid in a dose-dependent manner.

Hypothesis 4: Chitosan/ellagic acid composite films localize therapeutic effect via slow release of ellagic acid.

Hypothesis 5: Apoptosis and anti-angiogenesis are the mechanisms by which the composite films inhibit the growth of cancer cells.

\subsubsection{In vivo Anti-Cancer Activity of a Chitosan/Ellagic Acid Composite Film in a Glioma Brain Cancer Model}

Hypothesis 6: A chitosan/ellagic acid composite film inhibits tumor growth in vivo using a subcutaneous model.

\subsubsection{An Injectable Gel System for a Local Delivery of Ellagic Acid}

Hypothesis 7: A liquid chitosan formulation forms a heat-induced chitosan gel at body temperature.

Hypothesis 8: A thermo-sensitive chitosan gel is biocompatible and delivers anticancer activity of ellagic acid via a simple injection. 


\title{
CHAPTER 3. DEVEOPMENT OF CHITOSAN/ELLAGIC ACID FILMS AS A LOCAL DRUG DELIVERY SYSTEM TO INDUCE APOPTOTIC DEATH OF HUMAN MELANOMA CELLS *
}

\begin{abstract}
3.1 Summary
This study was designed to develop a local delivery of ellagic acid (EA) using a natural chitosan polymer for cancer treatment. Chitosan/ellagic acid (Ch/EA) composite films were fabricated according to various concentrations of $0,0.05,0.1,0.5$, and $1 \%$ (w/v) of ellagic acid and characterized using Fourier transform infrared spectroscopy (FTIR), X-ray diffraction (XRD), scanning electron microscopy (SEM), and contact angle measurement. The WM115 human melanoma cell line was used as a skin cancer model while HS68 human newborn fibroblast cell line was tested as a control. Anticancer activities of the composite films were evaluated by measuring the number of viable cells using MTS assay and apoptosis assay. With increasing concentration of ellagic acid, the composite films exhibited increasing amide and ester linkages and diffraction peaks of the crystallized ellagic acid. In addition, the increasing ellagic acid in composite films enhanced surface roughness and hydrophilicity. Viability of melanoma cells cultured on the composite films containing $0.5 \%(\mathrm{w} / \mathrm{v})$ of ellagic acid or higher was lower than that on chitosan film by at least 11 times for 3 days incubation. This result clearly demonstrated that $\mathrm{Ch} / \mathrm{EA}$ composite films have the localized anti-cancer activity in an ellagic acid concentration-dependent manner by inducing apoptotic cell death.
\end{abstract}

\subsection{Introduction}

It is well known that the consumption of fruits and vegetables containing phytochemical rich compounds is helpful to reduce the risk of cancer [Mertens-Talcott 2003, Stoclet 2004]. Such dietary substances include a variety of chemical compounds such as phenolic acids possessing a single ring structure and flavonoids holding several hydroxyl groups associated with phenolic groups [Scalbert 2000]. Ellagic acid is one of naturally occurring phenolic compounds and presents in fruits and nuts such as blueberries, red raspberries, pomegranates, walnuts, and grape seeds [Mullen 2003]. Ellagic acid has been reported to have anti-cancer properties via cell cycle arrest, induction of apoptosis, and inhibition of tumor formation and growth [Labrecque 2005, Losso 2004, Narayanan 1999, Whitley 2003].

In recent years, the implantable polymeric system has gained special attention in order to treat localized diseases or cancers in specific anatomical sites such as the skin [Castro 2003, Chen 2003], bone [Withrow 2004], brain [Dhanikula 2004, Ewend 1996,

\footnotetext{
* Adapted with permission of Wiley Periodicals. Kim S, Liu Y, Gaber MW, Bumgardner JD, Haggard WO, Yang Y. Development of chitosan-ellagic acid films as a local drug delivery system to induce apoptotic death of human melanoma cells. J Biomed Mater Res Part B: Appl Biomater. 2009;90B:145-155.
} 
Hammoud 2003], cervix [Keskar 2006], lung [Harper 1999], liver [Vog 1 2002], and bladder [Lu 2004]. Compared with conventional chemotherapy, these delivery systems are designed to maintain the sufficient drug dose at a local target site over the necessary duration. This local drug delivery has a potential to reduce the toxic effect of the drug to the surrounding tissues as well. Polymeric delivery systems include synthetic (e.g., poly (D, L-lactideco- glycolide), poly (ethylene-co-vinyl acetate), polyphosphoester, and HPMA) or natural (e.g., chitosan, collagen, and gelatin) polymers. They have been used in a variety of forms such as films, sponges, beads, gels, patches, and ointments.

Particularly, chitosan, a cationic natural polymer produced by alkaline Ndeacetylation of chitin, provides useful characteristics such as biocompatibility, biodegradability, film forming property with mechanical strength, low toxicity, and low cost [Jaworska 2003, Tien 2003]. It has been extensively used over a wide range of applications including drug carriers, wound-healing agents, and tissue regeneration [Prashanth 2005, Ravi Kumar 2006, Yuan Y 2007]. The important characteristics of chitosan are dependent on its molecular weight, degree of deacetylation, crytallinity, viscosity, pKa, and etc [Pillai 2009]. In acidic environments, positively charged free amino groups of chitosan can form complexes with negatively charged molecules altering its physicochemical characteristics [Chatelet 2001, Wang 2006]. A number of amino groups that are available for chemical reaction with anionic drug or polymeric systems provide promising biomedical applications. In addition, the uniqueness of chitosan among polysaccharides is its susceptibility to enzymatic depolymerization and supply of cell activating oligomers and $\mathrm{N}$-acetylglucosamine for rebuilding of other biological events [Stokke 1995].

Melanoma, the most deadly skin cancer, is a malignant tumor of melanocytes and the retinal pigment epithelial cells [Smith 2002]. Sun exposure has long been known as a main cause in the development of skin cancer because of its UVA $(320-400 \mathrm{~nm})$ and UVB (280-320nm) components causing DNA mutations [Hersey 2006, Brenner 2005]. Although the malignant melanoma in the early stage can be cured by surgery, there still remains a high risk of recurrence and rapid metastasis to other organs. Therefore, chemotherapy is mostly applied after surgery, but sometimes low therapeutic efficacy impacts the quality of the patient's [Demierre 2006, Hussein 2003]. In this regard, the targeting of the molecules such as p53 [Gartel 2002, Hussein 2003], Bcl-2 family proteins [Chen 2002, Rieber 2001], TRAIL-related molecules [Hersey 2006], and certain elements of the Fas pathway [Kim 2006] to induce the apoptosis will provide a new approach to treat the recurrent tumor. The induction of apoptosis in the melanoma generally involves the activation of a complex array of caspases through two principal pathways, which are the transmembrane extrinsic pathway and the mitochondrial intrinsic pathway. These two pathways can be amplified through mitochondrial damage [Hersey 2006]. The mechanism responsible for apoptosis in malignant melanoma is still unclear. However, apoptotic cell death via these therapeutic approaches would be expected to minimize inflammatory effects causing the damage in the surrounding tissue [Willingham 1999].

In the present study, the chitosan/ellagic acid (Ch/EA) composite films were developed and characterized in terms of chemical structure, crystallinity, and surface 
morphology. The biological effect of the composite films was evaluated using WM115 human melanoma and HS68 human newborn fibroblast. It was hypothesized that the $\mathrm{Ch} / \mathrm{EA}$ composite films impart an anti-proliferative effect against the melanoma cells by inducing apoptosis in an ellagic acid-concentration dependent manner.

\subsection{Materials and Methods}

\subsubsection{Materials}

The chitosan was purchased from Sigma Aldrich in medium molecular weight (190-310 kDa) with $\sim 85 \%$ of a degree of deacetylation. The ellagic acid was also obtained from Sigma Aldrich. Fetal bovine serum (FBS), trypsin-EDTA, L-glutamine, antibiotic-antimycotic, phosphate buffered saline (PBS), and Dulbecco's Modified Eagle Medium (DMEM) were obtained from Gibco ${ }^{\mathrm{TM}}$. The Annexin V-PE and 7-AAD (7amino-actinomycin D) staining solution were purchased from BD Biosciences. The WM115 human melanoma and HS68 human newborn fibroblast cell lines were purchased from and cultured as per American Type Culture Collection (ATCC) instructions.

\subsubsection{Preparation of Chitosan Films (Ch)}

A $1 \%(\mathrm{w} / \mathrm{v})$ chitosan solution was prepared by stirring powdered chitosan in $0.75 \%(\mathrm{v} / \mathrm{v})$ aqueous acetic acid at room temperature overnight. The insoluble particles in the chitosan solution were removed by filtration. The chitosan solution was dialyzed at room temperature using a dialysis membrane, which was placed in a container filled with the distilled water for 6 days to reduce the acidity attributed to acetic acid. The final $\mathrm{pH}$ value of the chitosan solution was around 6.6. The neutralized chitosan solution was autoclaved at $121^{\circ} \mathrm{C}$ for $20 \mathrm{~min}$ and cast in nonstick plates and dried by evaporation for 1 day. The dry transparent film was carefully peeled off from the plates, washed with distilled water to remove salt, and then air-dried in the biological hood.

\subsubsection{Preparation of Chitosan/Ellagic Acid (Ch/EA) Composite Films}

The ellagic acid solutions in the distilled water $(0.05,0.1,0.5$, and $1 \%(\mathrm{w} / \mathrm{v}))$ were prepared. The powdered ellagic acid was dissolved into the distilled water by adding several drops of $0.1 \mathrm{M} \mathrm{NaOH}$, with constant stirring at room temperature overnight. The $1 \%(\mathrm{w} / \mathrm{v})$ chitosan solutions were then mixed with the ellagic acid solutions at a chitosan to ellagic acid volume ratio of $1: 1$. The mixed $\mathrm{Ch} / \mathrm{EA}$ solutions were agitated at $40^{\circ} \mathrm{C}$ for 2 hours and were then neutralized for 3 or 4 days using dialysis membranes to reach the final $\mathrm{pH}$ values, ranging from 6.1 to 6.6 . The $\mathrm{Ch} / \mathrm{EA}$ solutions were autoclaved at $121^{\circ} \mathrm{C}$ for $20 \mathrm{~min}$ and cast in nonstick plates and dried by evaporation for 1 day in the biological hood. The chitosan/ellagic acid composite films (Ch/EA0.05, Ch/EA0.1, Ch/EA0.5, and 
$\mathrm{Ch} / \mathrm{EA} 1)$ were carefully peeled off from the plates, washed with distilled water to remove salt, and then air-dried in the biological hood.

\subsubsection{FTIR Spectra}

To characterize the surface functional groups of chitosan and $\mathrm{Ch} / \mathrm{EA}$ composite films (Ch/EA0.05, Ch/EA0.1, Ch/EA0.5, and $\mathrm{Ch} / \mathrm{EA} 1), \mathrm{FTIR}$ spectra were obtained using the BRUKER (TENSOR 27) spectrophotometer coupled to a PC with the analysis software. FTIR was used to investigate the surface chemical information according to different concentration of ellagic acid. The films were placed in the holder directly in the IR laser beam. All spectra were recorded by the transmittance mode (100 times scanning, 4000-800 $\mathrm{cm}^{-1}$ ).

\subsubsection{X-Ray Diffraction}

XRD was used to characterize the crystal structure of the films, which is related to mechanical strength and biodegradation rate. The XRD patterns were determined using an Advance D8 X-ray diffractometer (Bruker AXS, Madison, WI) and $\mathrm{K} \alpha \mathrm{Cu}$ radiation with the voltage of $40 \mathrm{kV}$ and the intensity of $40 \mathrm{~mA}$. The crystal structure of ellagic acid in powder and $\mathrm{Ch} / \mathrm{EA}$ composite films was examined by measuring the X-ray powder diffraction patterns. The data were collected over $2 \theta$ angular range from $4^{\circ}$ to $40^{\circ}$ in continuous mode with a scan rate of $0.75^{\circ} / \mathrm{min}$ and then X-ray diffractograms were displayed.

\subsubsection{Scanning Electron Microscopy}

The morphology of the films was observed by scanning electron microscope (JEOL, JSM-6460LV). SEM was used to investigate the different surface morphology of composite films according to different concentration of ellagic acid. The films were coated with a gold layer and examined at a $10 \mathrm{kV}$ accelerating voltage with a working distance of $11 \mathrm{~mm}$.

\subsubsection{Water Contact Angle Determination}

Water contact angle was used to determine wettability of $\mathrm{Ch} / \mathrm{EA}$ composite films. The water contact angle of the films was measured using the VCA OPTIMA from the AST Products (Boston, MA). The samples (five samples per group) were coated onto glass slides and placed on the sample stage. The sessile drop method was used to measure the contact angle of the samples immediately. Distilled water droplets were dropped at five different sites on each slide sample, and then the mean and standard deviation values were calculated. All measurements were performed at ambient temperature. 


\subsubsection{Cell Culture}

The WM115 human melanoma cell line and HS68 human newborn fibroblast cell line were grown and maintained in DMEM media with $10 \%$ FBS, $1 \%$ antibiotic/antimycotic mixture, $5 \mathrm{~mL}$ of L-glutamine $(200 \mathrm{mM})$, and sodium pyruvate. The cells were cultured in an incubator supplied with $5 \% \mathrm{CO}_{2}$ at $37^{\circ} \mathrm{C}$. The culture medium was changed every 3 days.

\subsubsection{Cell Viability-Direct Contact}

To determine the biological effect of the $\mathrm{Ch} / \mathrm{EA}$ composite films on the two cell lines, the numbers of viable cells (melanoma and fibroblasts) were measured by MTS assay, and the cell morphology was observed by microscope (Nikon ECLIPSE TE-2000U). $150 \mu \mathrm{l}$ of the sterilized samples was coated onto each well of 48-well plates and dried at room temperature, followed by rinsing with PBS ( $\mathrm{pH} 7.4)$ twice, and then dried again. The cells were seeded on each sample at a density of 30,000 cells per well and cultured without medium exchange for 4, 24, and 72 hours. At the end of the incubation period, the cell viability was determined with the Cell Titer 96AQueous One Solution Cell Proliferation Assay (MTS assay, Promega Corp.). Each $60 \mu \mathrm{L}$ of Cell Titer 96AQueous One Solution was added into each well of the 48-well plate containing the samples in 300 $\mu \mathrm{L}$ of culture medium. Following an incubation period of 2 hours at $37^{\circ} \mathrm{C} 5 \% \mathrm{CO}_{2}, 100$ $\mu \mathrm{L}$ of solution in each well of the 48-well plate was transferred into the 96-well assay plates and the light absorbance at $490 \mathrm{~nm}$ was recorded using a micro-plate reader (Molecular Device Vmax kinetic). The background absorbance at $490 \mathrm{~nm}$ was corrected by subtracting the average absorbance of the control wells without cells from each well to estimate viable cellular activity. Prior to the MTS assay, the cell morphology was documented using an inverted bright field (BF) microscope (Nikon ECLIPSE TE-2000U) and processed using MetaVue software.

\subsubsection{Cell Viability-Indirect Contact}

The indirect culture via BD BioCoat ${ }^{\mathrm{TM}}$ Control Cell Culture inserts (12-well formats) was used to evaluate the effect of the $\mathrm{Ch} / \mathrm{EA}$ composite films on cell viability via cell culture media. The insert contains a $0.45-\mu \mathrm{m}$ pore size PET membrane with a thin layer of basement membrane matrix, which prevents cells from migrating through the membrane, and allows only the culture medium to permeate through the PET membrane. The bottom of each well plate was coated with $700 \mu \mathrm{L}$ of the sterilized samples and dried at room temperature, followed by rinsing with PBS ( $\mathrm{pH} 7.4)$ twice, and then dried again. Cell culture medium $(1.7 \mathrm{~mL})$ was added into each well of the 12-well plate with inserts, which were allowed to hydrate for 2 hours at $37^{\circ} \mathrm{C}$. The melanoma cells were then seeded at a density of 60,000 cells per well on the upper chamber with $0.3 \mathrm{~mL}$ of the culture medium. After the incubation for 4, 24, and 72 hours, the number of viable cells was determined using the Cell Titer 96AQueous One Solution according to the manufacturer's instructions. 


\subsubsection{Cell Viability-at the Interface of the Presence and Absence of the Material}

The localized effect of the Ch/EA composite film on cells was evaluated by observing the cellular behavior at the interface of the presence and absence of the material. The chitosan solution containing $1 \%(\mathrm{w} / \mathrm{v})$ of ellagic acid $(\mathrm{Ch} / \mathrm{EA} 1)$ was coated on the half area of each 12-well plate. The cells were then seeded into the 12-well plate. At 4, 24, and 72 hours incubation, the cell morphology at the boundary between the nontreated culture plate and the $\mathrm{Ch} / \mathrm{EA} 1$ was observed using a microscope (Nikon ECLIPSE TE-2000U).

\subsubsection{Apoptosis Assay}

To verify the statement that the $\mathrm{Ch} / \mathrm{EA}$ composite films inhibit the cancer cell growth via induction of apoptosis, Annexin V-PE and 7-AAD (7-amino-actinomycin D) staining was performed. The phospholipids phosphatidylserine (PS) on the membrane of the apoptotic cells is translocated from the inner to the outer leaflet of the plasma membrane resulting in exposing PS to the external cellular environment. The Annexin V detects the PS on the outer plasma membrane at the early stage of apoptosis, whereas the 7-AAD evaluates the progressive loss of the membrane permeability and discriminates apoptosis at the end stage of the apoptotic or dead cells [Kuypers 1996]. The early apoptotic cells are the Annexin V-PE positive and the 7-AAD negative, whereas the late apoptotic or dead cells are both V-PE and 7-AAD positive [Chen 2002, Kim 2006]. The melanoma cells were seeded on the 6-well plates coated with Ch/EA0.5 at a density of 500,000 cells per well. The cells were incubated for 2 and 24 hours. At the end of the designated incubation period, both the floating and adherent cells were collected by trypsin, rinsed with the ice-cold PBS, and transferred into a $15 \mathrm{~mL}$ capacity centrifuge tube. After centrifugation at 1,200 rpm for $10 \mathrm{~min}$, the cells were rinsed with ice-cold PBS, centrifuged again, and then resuspended. Subsequently, $100 \mu \mathrm{L}$ of the solution containing 100,000 cells was transferred into a $5 \mathrm{~mL}$ culture tube before $5 \mu \mathrm{L}$ of the Annexin V-PE and 7-AAD were added by gentle vortexing. After incubating the $5 \mathrm{~mL}$ culture tube for $15 \mathrm{~min}$ at room temperature in the dark, $400 \mu \mathrm{L}$ of the binding buffer was added prior to the analysis. The apoptotic cells were determined using the Flow Cytometer (Becton-Dickson FACS Caliber). The total percentage of the apoptotic cells was calculated and the results were expressed as mean \pm SD.

\subsubsection{Statistical Analysis}

All data are presented as mean \pm standard deviation. Significant differences in cell viability and apoptosis were analyzed by one-way ANOVA test. The differences in groups and experimental time points were considered significant if $p<0.05$. 


\subsection{Results}

\subsubsection{FTIR Spectra}

The FTIR spectra obtained from the chitosan film and $\mathrm{Ch} / \mathrm{EA}$ composite films are shown in Figure 3.1. The spectrum of the chitosan film $(\mathrm{Ch})$ exhibited several characteristic peaks which were attributed to the $\mathrm{C}=\mathrm{O}$ stretch of amide $\mathrm{I}$ bond at 1652 and $1559 \mathrm{~cm}^{-1}$, the N-H bending vibrations of amide II at 1409 and $1381 \mathrm{~cm}^{-1}$, and the C-N bending of the amine groups at $1323 \mathrm{~cm}^{-1}$. The peaks at 1075 and $1036 \mathrm{~cm}^{-1}$, representative of the $\mathrm{C}-\mathrm{O}-\mathrm{C}$ bending, were observed. Compared with the chitosan film, the spectra of the $\mathrm{Ch} / \mathrm{EA}$ composite films showed greater intensity of the peaks at 1563 and $1352 \mathrm{~cm}^{-1}$, which were assigned to the $\mathrm{C}=\mathrm{O}$ stretch of the amide $\mathrm{I}$ bond and the $\mathrm{N}-\mathrm{H}$ bending vibrations of the amide II, respectively. Furthermore, a new peak attributed to the aromatic $\mathrm{C}=\mathrm{C}$ stretching was observed at $1507 \mathrm{~cm}^{-1}$. With increasing concentration of ellagic acid (Ch/EA0.5), the intensity of amine groups of the chitosan film decreased at $1330 \mathrm{~cm}^{-1}$. In addition, a strong ester linkage because of the $\mathrm{C}=\mathrm{O}$ stretch appeared around $1060 \mathrm{~cm}^{-1}$.

\subsubsection{X-Ray Diffraction}

XRD patterns of the ellagic acid in the powder and the Ch/EA composite films were used to determine the differences in the crystal structures. Several strong diffraction peaks were observed in the powdered ellagic acid at the following $2 \theta$ of $9.6^{\circ}, 10.5^{\circ}, 12.3^{\circ}$, $13.6^{\circ}, 17.5^{\circ}, 20.7^{\circ}, 25.1^{\circ}, 26.4^{\circ}, 28.3^{\circ}, 32.1^{\circ}, 34.7^{\circ}, 35.4^{\circ}, 36.9^{\circ}, 37.6^{\circ}$, and $39.1^{\circ}$ (Figure $3.2 \mathrm{a}$ ). The diffractogram of a neutralized chitosan film displayed two major crystalline peaks at $2 \theta$ of $10.5^{\circ}$ and $21^{\circ}$ with a characteristic broad hump as shown in Figure $3.2 \mathrm{~b}(1)$. The $\mathrm{Ch} / \mathrm{EA} 0.05$ and $\mathrm{Ch} / \mathrm{EA} 0.1$ became amorphous and did not show any reflective peaks, but exhibited a broad halo at $2 \theta$ of around $23^{\circ}$ as shown in Figure $3.2 \mathrm{~b}(2)$ and (3). With increasing concentration of ellagic acid as shown in Figure $3.2 \mathrm{~b}(4)$ and (5), several diffraction peaks were observed in the Ch/EA0.5 at $2 \theta$ of $9.6^{\circ}, 12.3^{\circ}$, $13.6^{\circ}$ and in the $\mathrm{Ch} / \mathrm{EA} 1$ at $2 \theta$ of $9.6^{\circ}, 12.3^{\circ}, 13.6^{\circ}, 17.5^{\circ}, 28.3^{\circ}$. This result demonstrated that the newly appeared peaks were assigned to the crystal structure of ellagic acid.

\subsubsection{Scanning Electron Microscopy}

Figure 3.3 displays the SEM micrographs of the $\mathrm{Ch} / \mathrm{EA}$ composite films. The morphological difference was observed on the surface of each film. The surface morphology of $\mathrm{Ch} / \mathrm{EA} 0.05$ (Figure 3.3a) was fairly smooth and almost similar to that of the chitosan film. However, the surface morphologies of the other films became rougher and more heterogeneous with increasing concentration of ellagic acid as shown in Figure 3.3b-d. 


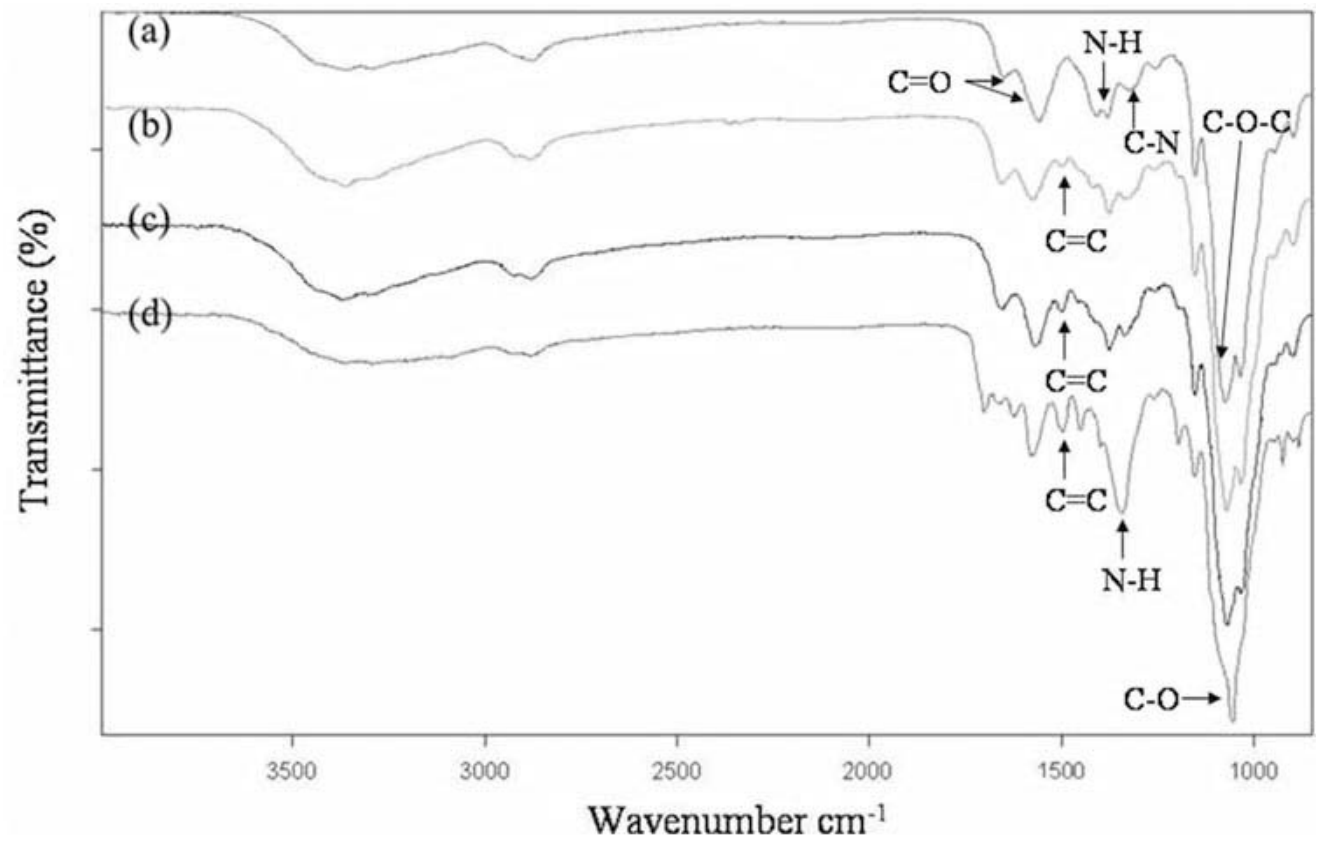

Figure 3.1 FTIR spectra of the chitosan/ellagic acid composite films with varying concentration of the ellagic acid. (a) Ch (neutralized chitosan film); (b) Ch/EA0.05; (c) $\mathrm{Ch} / \mathrm{EA} 0.1 ;$ (d) $\mathrm{Ch} / \mathrm{EA} 0.5$. 


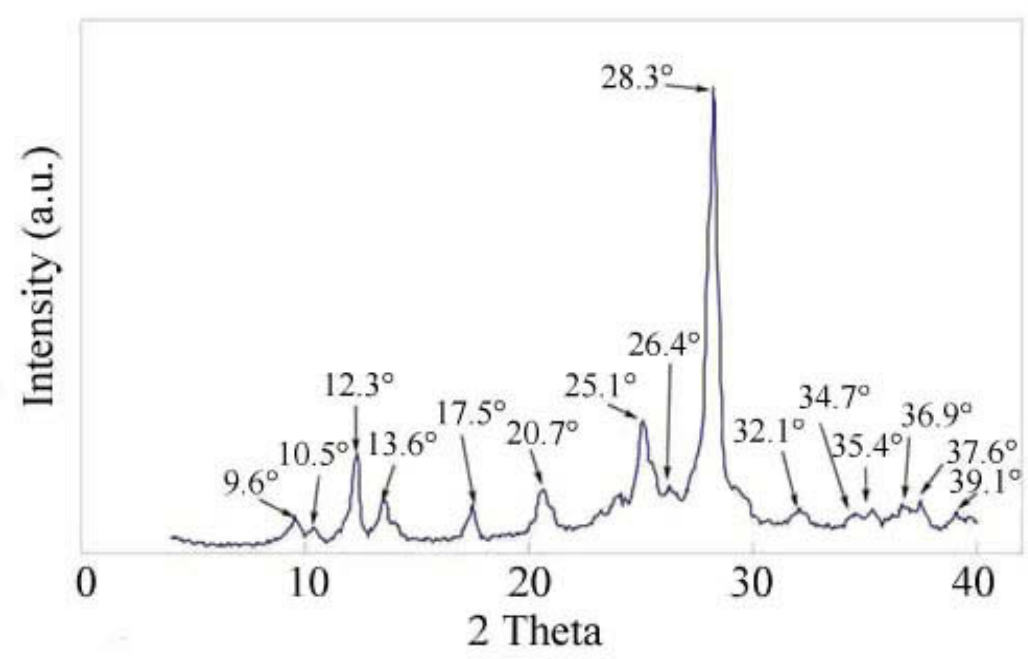

(a)

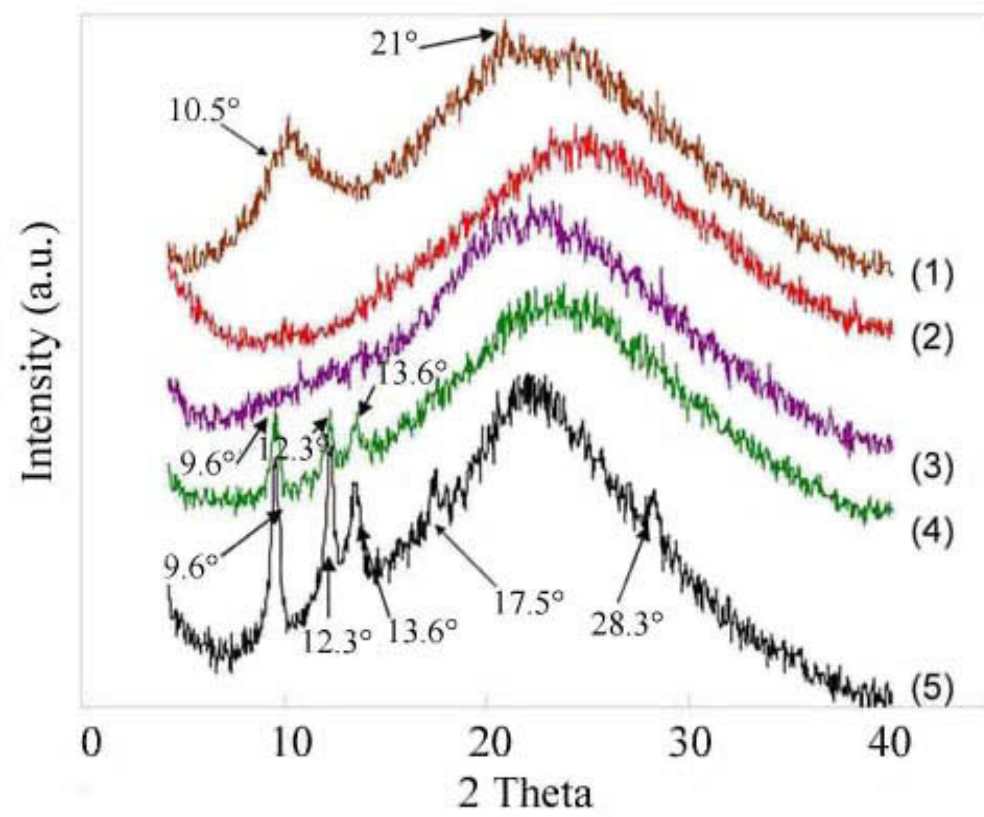

(b)

Figure $3.2 \mathrm{X}$-ray diffraction patterns. (a) the ellagic acid in powder and (b) the chitosan/ellagic acid composite films with varying concentration of the ellagic acid. (1) Ch (neutralized chitosan film); (2) Ch/EA0.05; (3) Ch/EA0.1; (4) Ch/EA0.5; (5) Ch/EA1 at ambient temperature. 


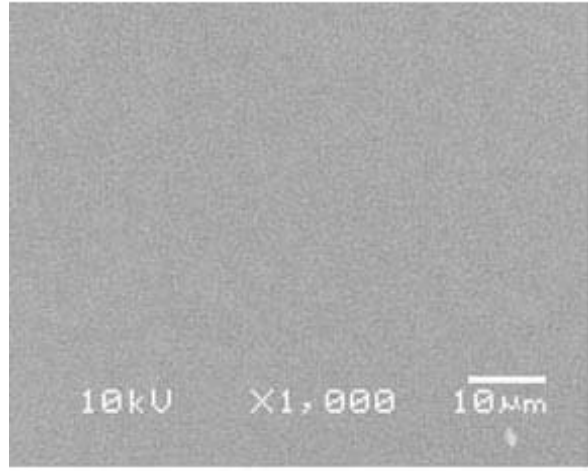

(a)

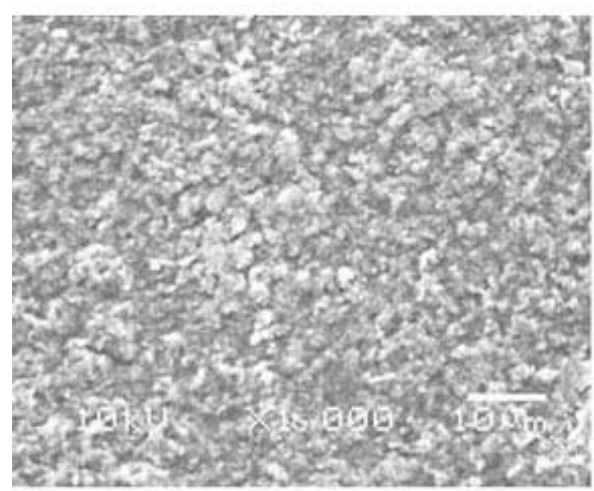

(c)

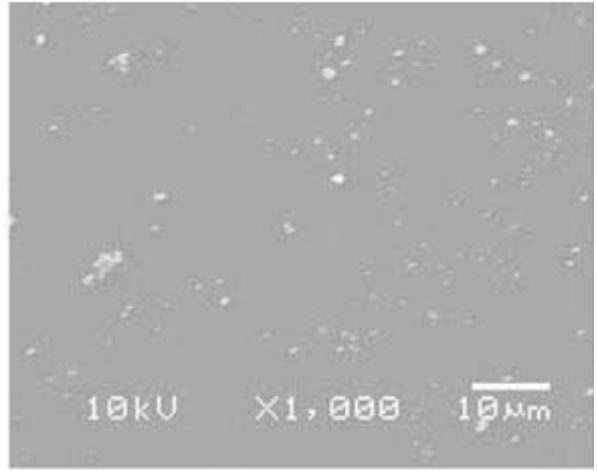

(b)

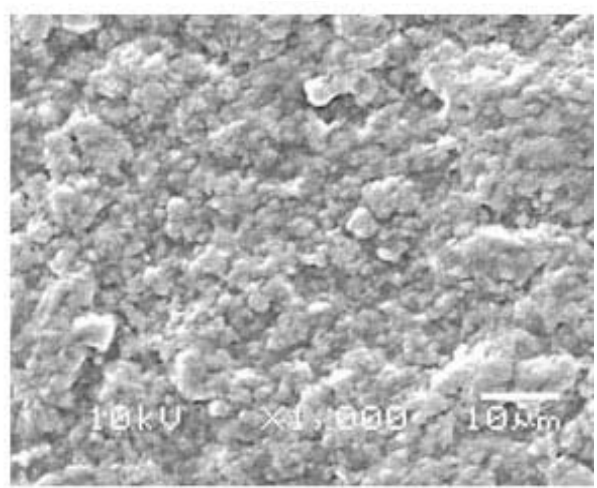

(d)

Figure 3.3 SEM micrographs on the surface of the chitosan/ellagic acid composite films with varying concentration of the ellagic acid. (a) $\mathrm{Ch} / \mathrm{EA} 0.05$; (b) $\mathrm{Ch} / \mathrm{EA} 0.1$; (c) $\mathrm{Ch} / \mathrm{EA} 0.5 ;(\mathrm{d}) \mathrm{Ch} / \mathrm{EA} 1 .(\mathrm{MAG}=\times 1000)$ 


\subsubsection{Water Contact Angle Determination}

The effect of concentration of ellagic acid on the hydrophilicity of the $\mathrm{Ch} / \mathrm{EA}$ composite films is shown in Table 3.1. The measurement of water contact angle was performed to study the wettability of the composite films containing various concentration of ellagic acid. The water contact angles on the composite films decreased with increasing concentration of the ellagic acid. This result indicated that the surface of the composite films became more hydrophilic with increasing concentration of ellagic acid and rougher surface shown in Figure 3.3c and d.

\subsubsection{Cell Viability-Direct Contact}

We evaluated the effect of the Ch/EA composite films on the growth of the melanoma cells and fibroblasts by microscopy and MTS assay. The microscopic observation shows the morphological changes of the WM115 human melanoma cells (Figure 3.4) and HS68 human newborn fibroblasts (Figure 3.5) on $\mathrm{Ch} / \mathrm{EA}$ composite films at 4 and 72 hours of incubation, respectively. The melanoma cells first adhered to all the films in round shape at 4 hours of the incubation as shown in Figure 3.4a-f. After 72 hours of the incubation, the melanoma cells appeared to aggregate on the chitosan film, $\mathrm{Ch} / \mathrm{EA} 0.05$, and $\mathrm{Ch} / \mathrm{EA} 0.1$ as shown in Figure 3.4h-j. However, as shown in Figure 3.4k and 1 , the melanoma cells retained their round shaped on the Ch/EA0.5 and Ch/EA1 and the aggregation of the cells did not occur for 3 days of cell culture.

Similarly, the fibroblasts first adhered to all the films in a round shape at 4 hours of the incubation as shown in Figure 3.5a-f. After 72 hours of the incubation, the fibroblasts changed their morphologies from circular to spindle shaped on the chitosan film, $\mathrm{Ch} / \mathrm{EA} 0.05$, and $\mathrm{Ch} / \mathrm{EA} 0.1$ as shown in Figure 3.5h-j. This observation indicates the growth and proliferation of the cells. However, the fibroblasts still exhibited a round shape on the $\mathrm{Ch} / \mathrm{EA} 0.5$ and $\mathrm{Ch} / \mathrm{EA} 1$ as shown in Figure $3.5 \mathrm{k}$ and 1.

In addition to a morphological change, there were significant increases in the cell viability of the melanoma (Figure 3.6a) on the chitosan film and Ch/EA0.05 after 24 and 72 hours of the incubation compared with 4 hours $(p<0.05)$. However, the cell viability was slightly decreased on the $\mathrm{Ch} / \mathrm{EA} 0.05$ after 72 hours of the incubation compared with 24 hours, but no significant change was observed $(p=0.17)$.

Table 3.1 Water contact angles of the chitosan/ellagic acid composite films.

\begin{tabular}{lccccc}
\hline & Chitosan & Ch/EA0.05 & Ch/EA0.1 & Ch/EA0.5 & Ch/EA1 \\
\hline $\begin{array}{c}\text { Contact } \\
\text { angle }\end{array}$ & $103.62 \pm 3.9$ & $99.87 \pm 3.16$ & $97.39 \pm 2.35$ & $94.75 \pm 4.4$ & $88.02 \pm 2.61$ \\
\hline
\end{tabular}


Figure 3.4 Photomicrographs of WM115 melanoma cells cultured on the chitosan coatings containing various concentration of ellagic acid. (a) no treatment; (b) chitosan film; (c) Ch/EA0.05; (d) Ch/EA0.1; (e) Ch/EA0.5; (f) Ch/EA1 for 4 hours of the incubation. (g) no treatment (tissue culture plate); (h) chitosan film; (i) Ch/EA0.05; (j) $\mathrm{Ch} / \mathrm{EA} 0.1$; (k) $\mathrm{Ch} / \mathrm{EA} 0.5$; (1) $\mathrm{Ch} / \mathrm{EA} 1$ for 72 hours of the incubation. The initial cell density was 30,000 cells per well. $(\mathrm{MAG}=\times 100)$. Arrows indicate cells on the coating material. 


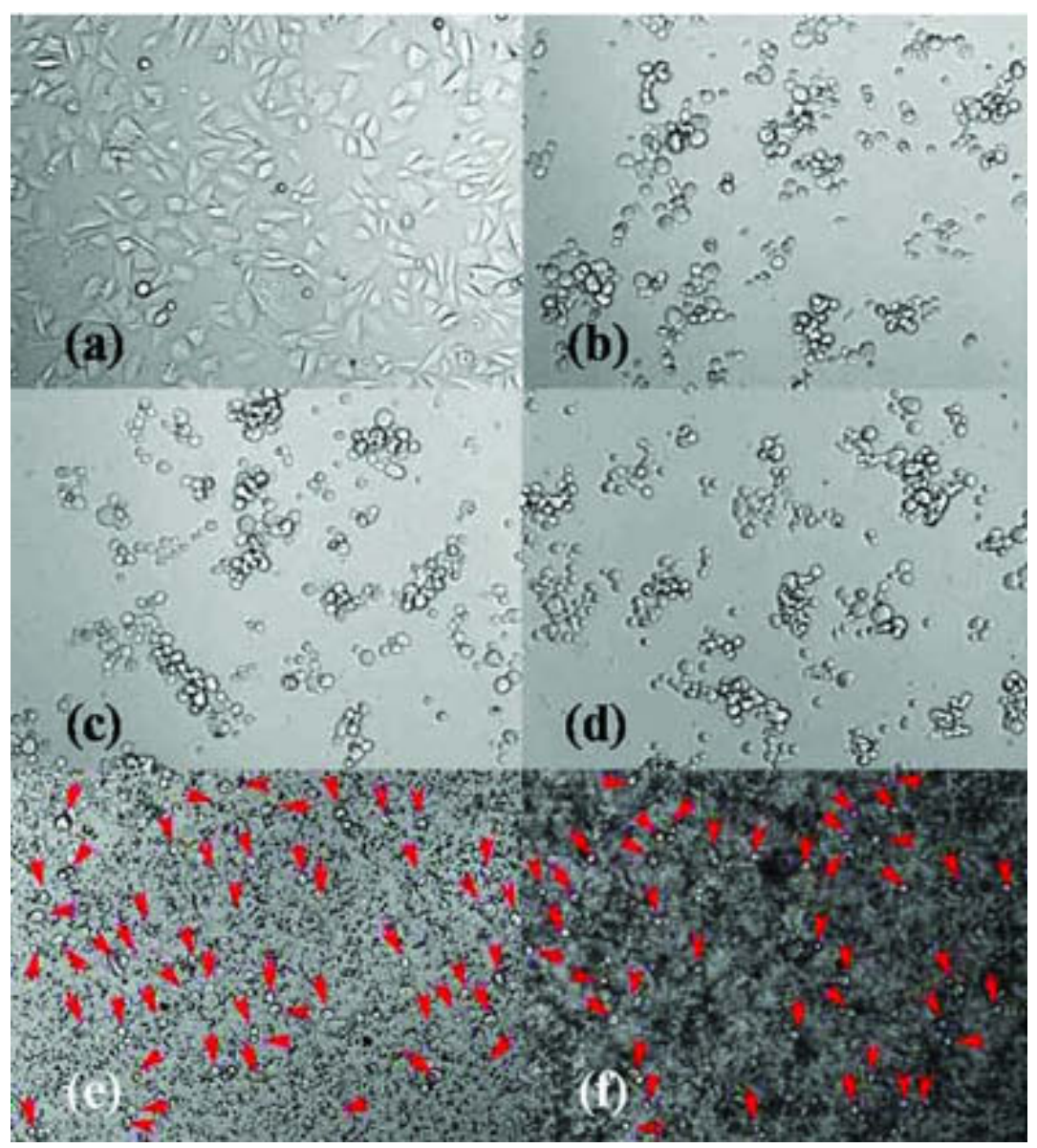




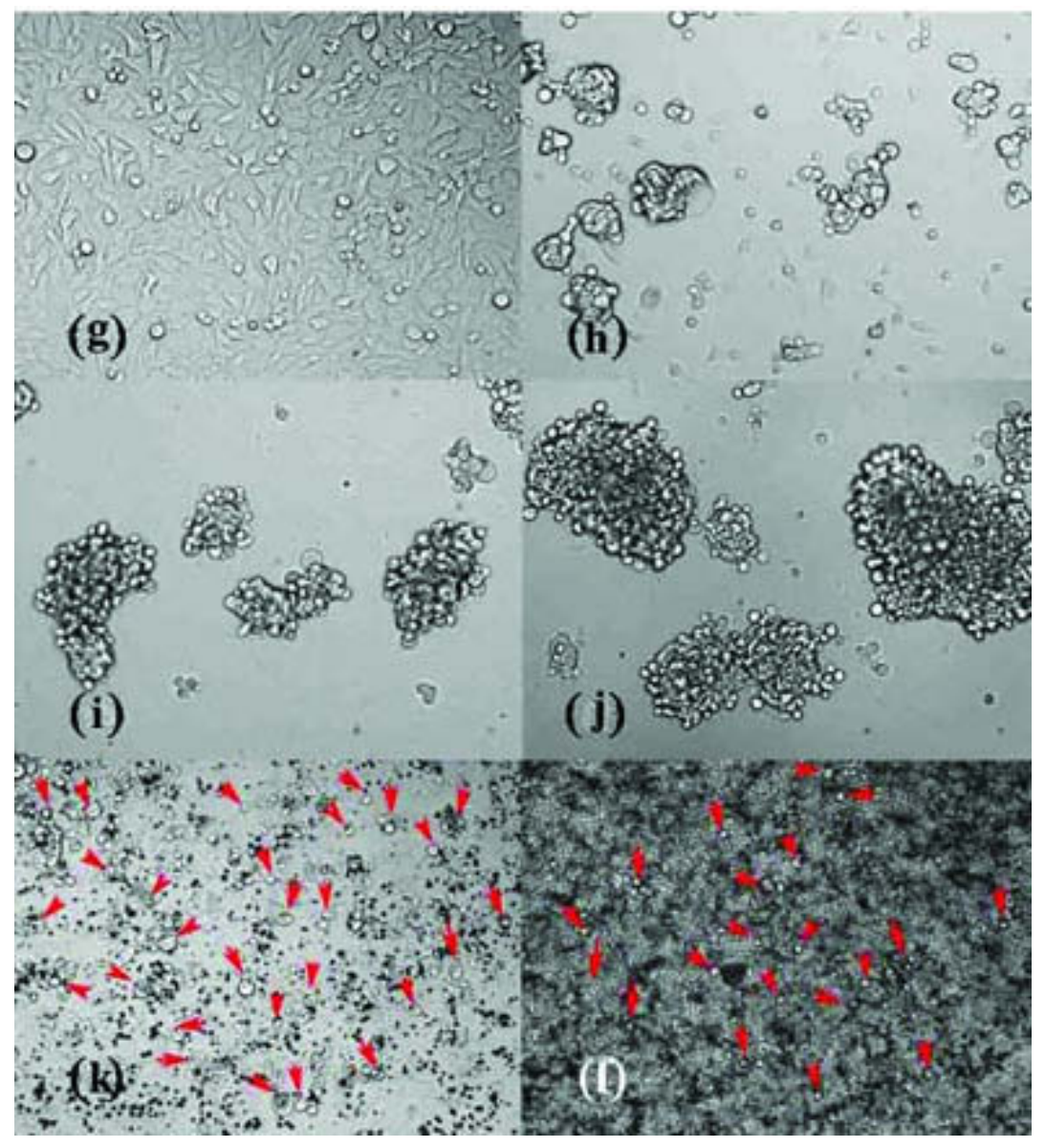

Figure 3.4 (continued). 
Figure 3.5 Photomicrographs of HS68 fibroblasts cultured on the chitosan coatings containing various concentration of ellagic acid. (a) no treatment; (b) chitosan film; (c) $\mathrm{Ch} / \mathrm{EA} 0.05$; (d) Ch/EA0.1; (e) Ch/EA0.5; (f) Ch/EA1 for 4 hours of the incubation. (g) no treatment (tissue culture plate); (h) chitosan film; (i) Ch/EA0.05; (j) Ch/EA0.1; (k) $\mathrm{Ch} / \mathrm{EA} 0.5$; (1) $\mathrm{Ch} / \mathrm{EA} 1$ for 72 hours of the incubation. The initial cell density was 30,000 cells / well. $(\mathrm{MAG}=\times 100)$ Arrows indicate cells on the coating material. 


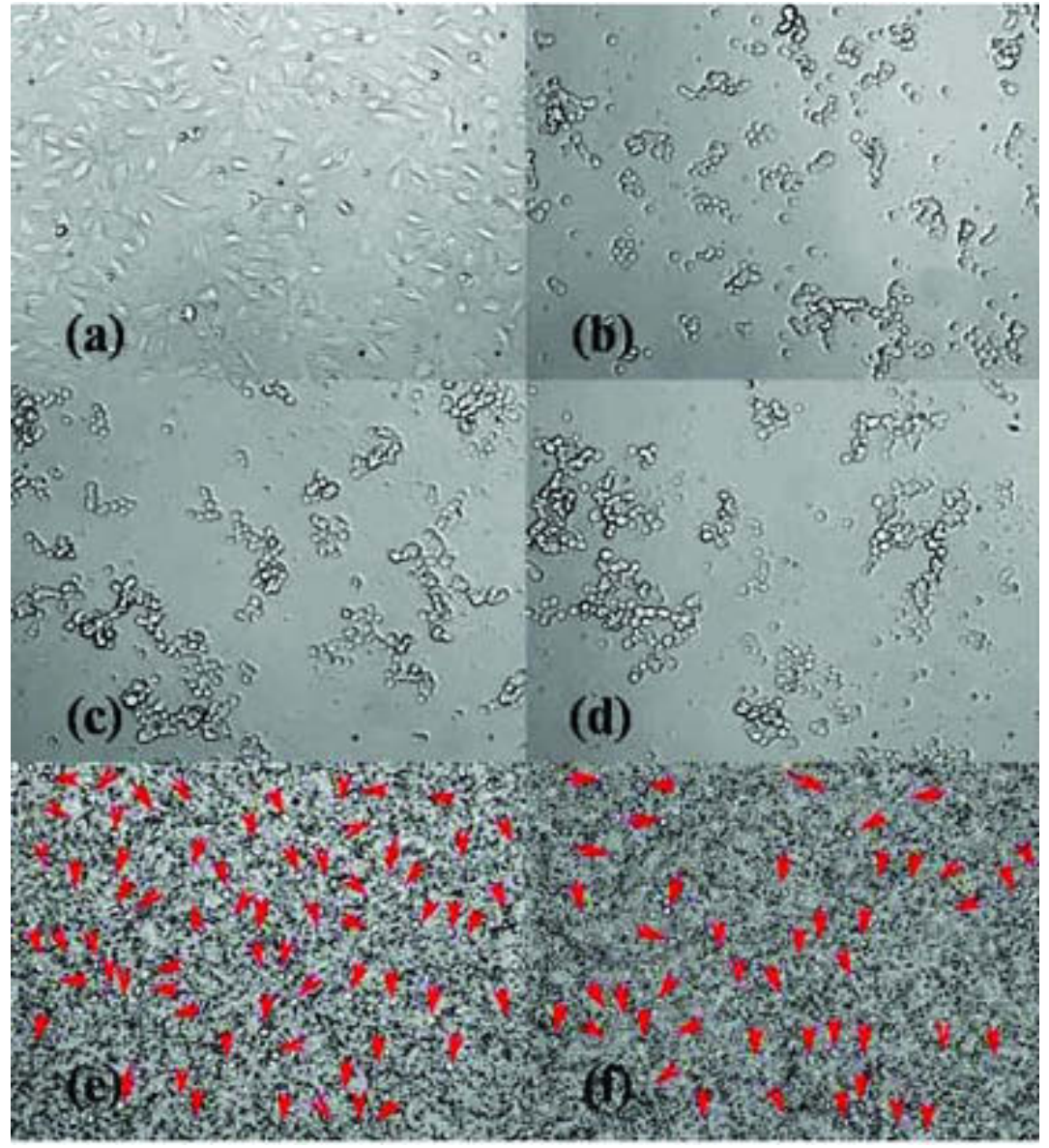




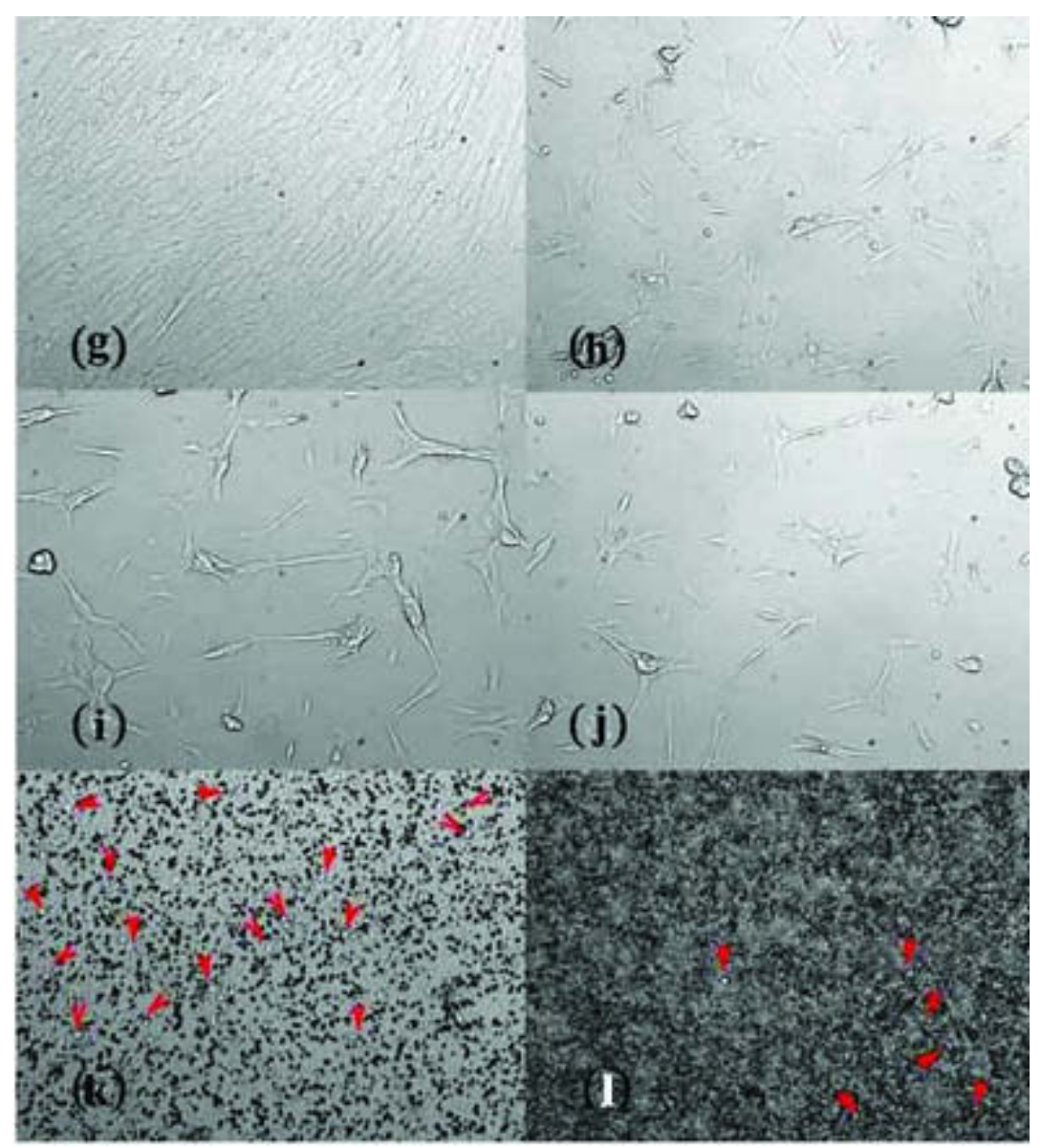

Figure 3.5 (continued). 


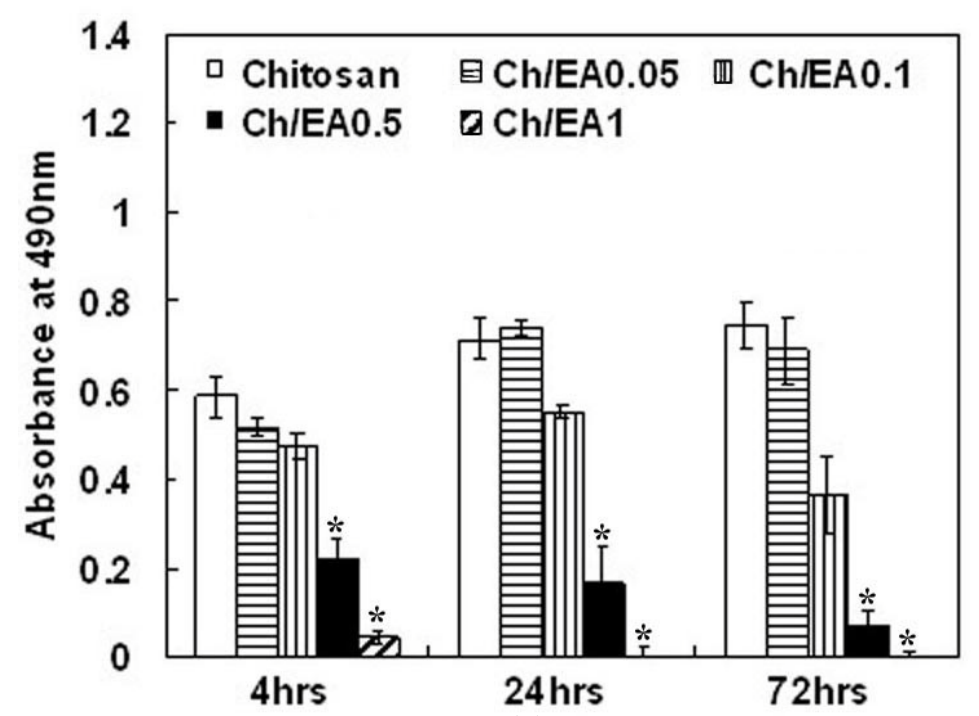

(a)

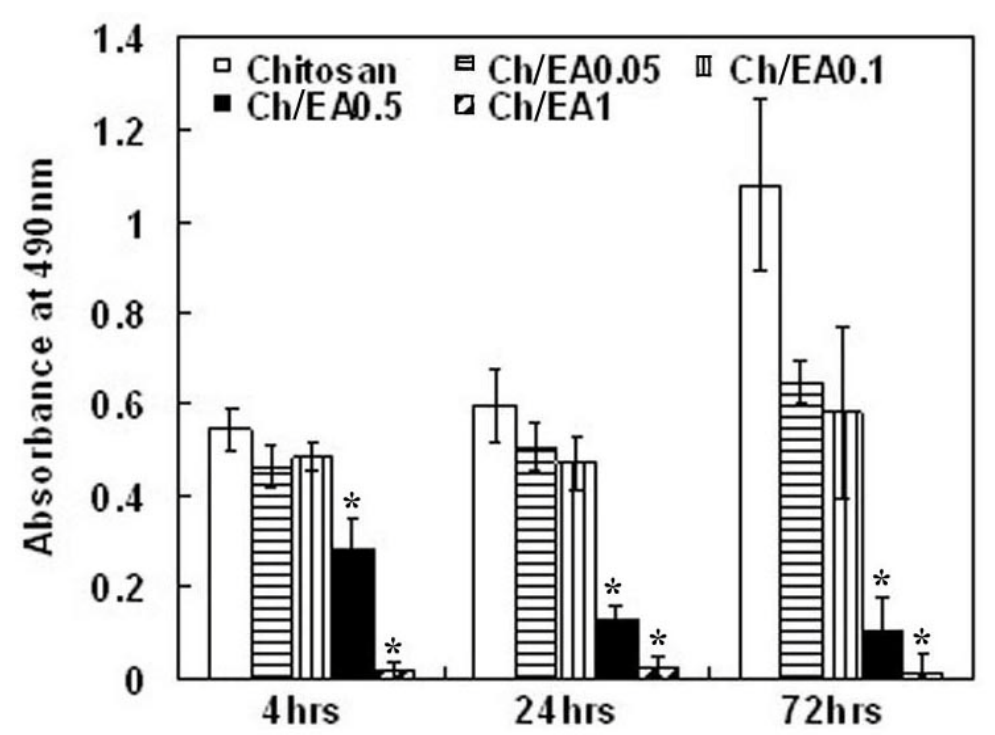

(b)

Figure 3.6 Viability of (a) WM115 melanomas and (b) HS68 fibroblasts via direct contact culture by MTS assay. The cells were cultured directly on each sample at a density of 30,000 cells per well without changing medium for 4,24 , and 72 hours. The absorbance expressed as a measure of cell viability on the chitosan coatings with varying concentration of ellagic acid for 3 days incubation: The initial cell density was 30,000 cells per well, respectively. $*$ denotes significantly different cell viability compared with chitosan film, Ch/EA0.05, and Ch/EA0.1 at 4, 24, and 72 hours incubation $(p<0.05)$. Each value represents the mean $\pm \mathrm{SD}(\mathrm{n}=6$ per each group). 
The significant decreases in the melanoma cell viability on the $\mathrm{Ch} / \mathrm{EA} 0.1$ (Figure 3.6a) were observed after 72 hours compared with 4 and 24 hours $(p<0.05)$, suggesting the inhibitory effect of the $\mathrm{Ch} / \mathrm{EA} 0.1$ on the melanoma cells. For fibroblasts cultured on the composite films (Figure 3.6b), there were significant increases in cell viability on the chitosan film and $\mathrm{Ch} / \mathrm{EA} 0.05$ after 72 hours of the incubation compared with 4 and 24 hours $(p<0.05)$. The cell viability on the $\mathrm{Ch} / \mathrm{EA} 0.1$ after 72 hours incubation slightly increased compared with 4 hours, but no significant changes were observed $(p=0.24)$.

As shown in Figure 3.6a and b, on the surface of the Ch/EA0.5 and Ch/EA1, the numbers of viable cells including fibroblasts and melanoma were found to decrease significantly over the 72 hours of the incubation $(p<0.05)$. In addition, both the cells on the $\mathrm{Ch} / \mathrm{EA} 0.5$ and $\mathrm{Ch} / \mathrm{EA} 1$ exhibited significantly lower viability compared with the cells on the chitosan film, $\mathrm{Ch} / \mathrm{EA} 0.05$, and $\mathrm{Ch} / \mathrm{EA} 0.1$ at 4, 24, and 72 hours of the incubation.

\subsubsection{Cell Viability-Indirect Contact}

The indirect contact culture was used to evaluate the effect of the released ellagic acid from the $\mathrm{Ch} / \mathrm{EA}$ composite films into the cell culture medium on the viability of the melanoma cells for 4, 24, and 72 hours of incubation, respectively. Figure 3.7 shows that the melanoma cells in the absence of direct contact significantly proliferated regardless of the concentration of the ellagic acid after 72 hours of incubation compared with 4 hours $(p<0.05)$. However, the melanoma cells in the absence of direct contact with the $\mathrm{Ch} / \mathrm{EA} 1$ exhibited significantly lower cell viability compared with the chitosan film at 72 hours of incubation $\left({ }^{*} p<0.05\right)$. This result reflected the inhibitory effect of high concentration of ellagic acid on cell proliferation in the absence of direct contact. There are no significant differences among other $\mathrm{Ch} / \mathrm{EA}$ composite film groups.

\subsubsection{Cell Viability at the Interface of the Presence and Absence of the Material}

In addition, Figure 3.8 shows the cellular morphology at the boundary between the non-treated culture plate control and the $\mathrm{Ch} / \mathrm{EA} 1$. Both the melanoma cells (Figure 3.8a) and fibroblasts (Figure 3.8b) on the non-treated culture plate close to the film areas were viable, well spread, and proliferated for 3 days, whereas the cells on the film areas did not change in morphology and the cell number decreased. These photomicrographs demonstrated that cell viability was significantly affected by direct contact of the composite films. This result suggests that the targeted area can be more effectively treated than the surrounding area.

\subsubsection{Apoptosis Assay}

Generally, cell viability is determined by the mechanism of necrosis or apoptosis. Necrosis is the result of the destruction of the plasma membrane by external damage. On the other hand, apoptosis, which is much slower than necrosis, leads to highly controlled 


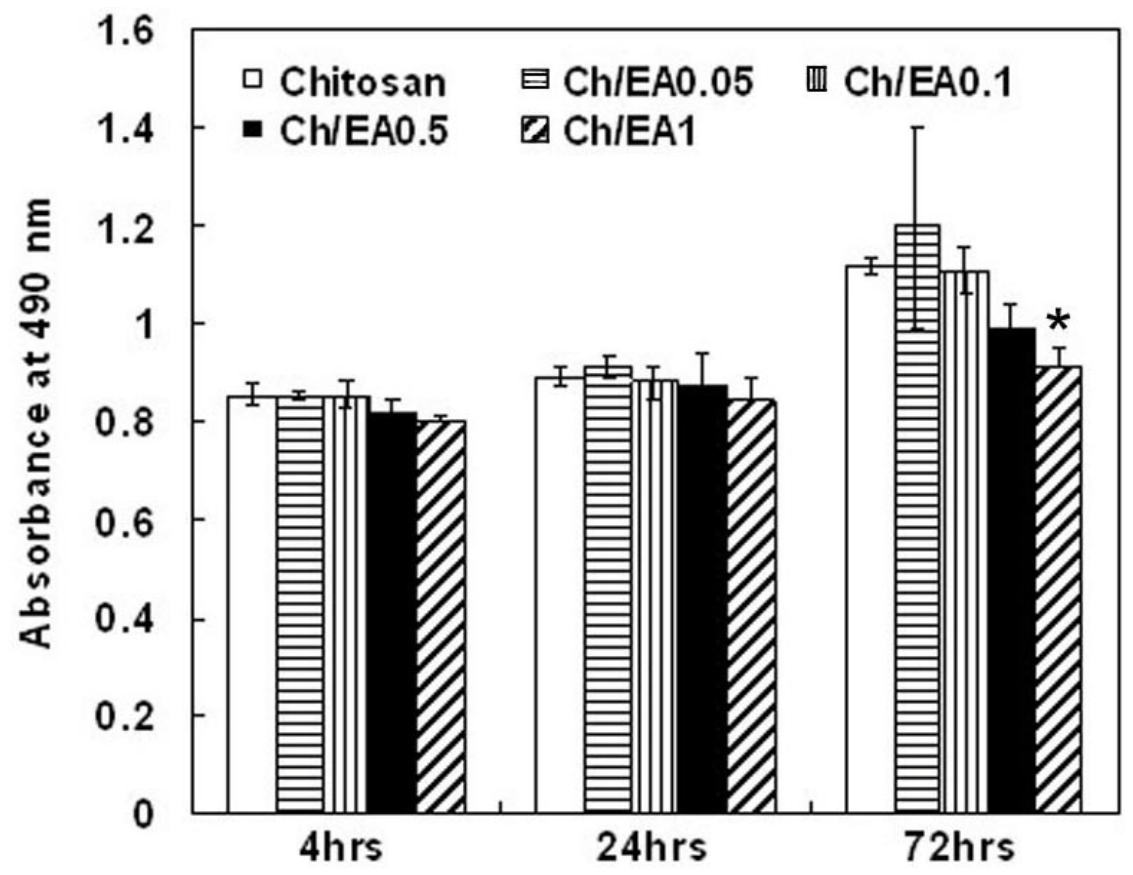

Figure 3.7 Viability of WM115 melanomas via indirect contact culture by MTS assay. The cells seeded onto the permeable PET membrane were affected by the released ellagic acid into the culture medium. The absorbance expressed as a measure of WM115 cell viability from each sample for the time point indicated; initially, the cell density was 60,000 cells per well. * denotes significantly different cell viability compared with the chitosan film at 72 hours incubation $(p<0.05)$. Each value represents the mean \pm SD $(\mathrm{n}=$ 4 per each group). 


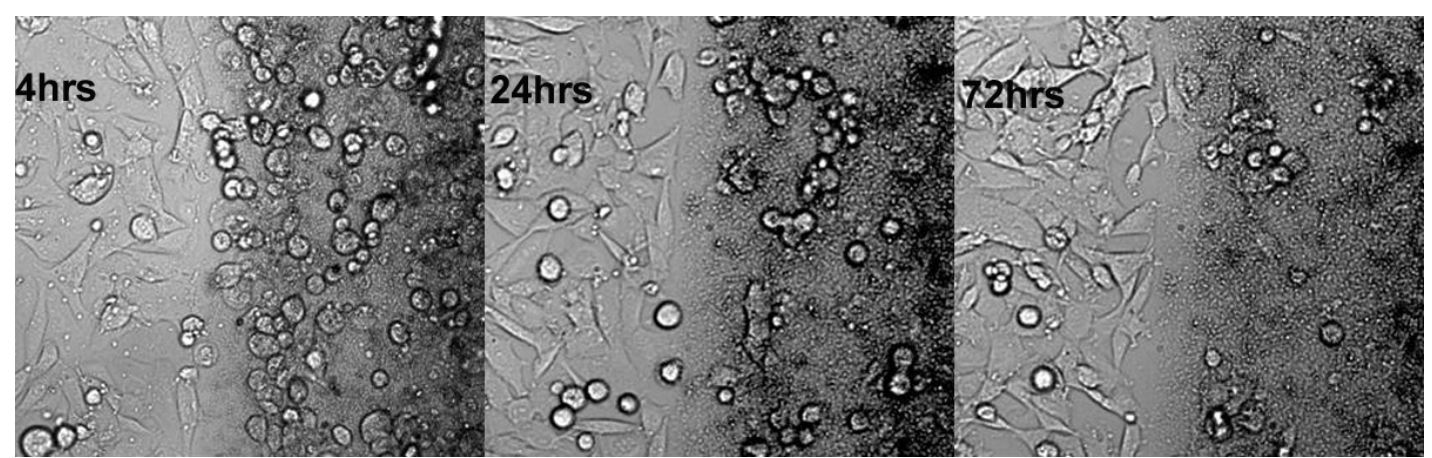

(a)

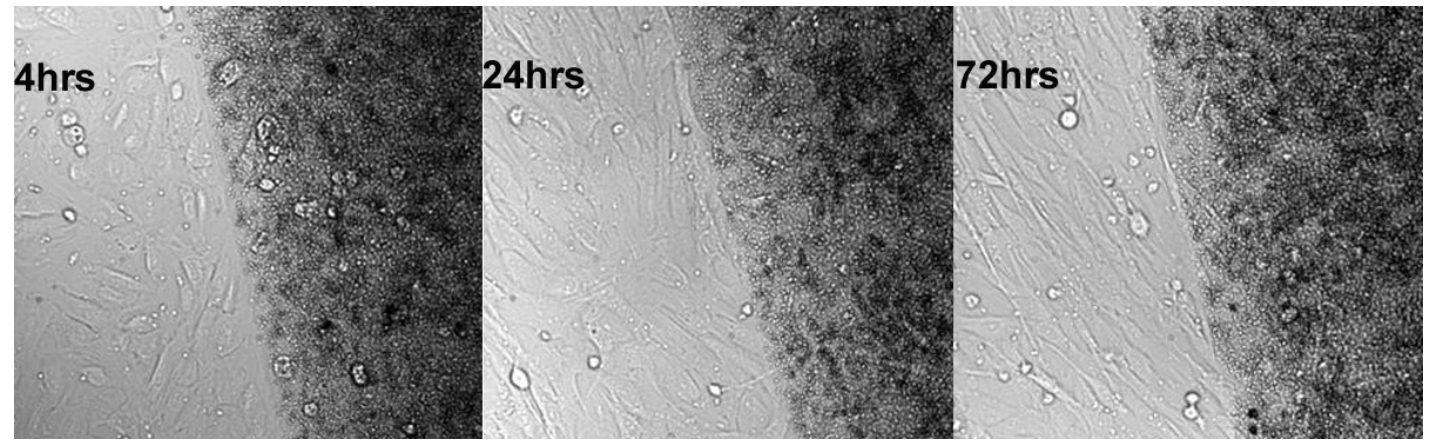

(b)

Figure 3.8 Photomicrographs of (a) WM115 melanomas and (b) HS68 fibroblasts at the boundary between the absence and presence of $\mathrm{Ch} / \mathrm{EA} 1$. The dark area indicates the cell morphology on the coating materials, and the bright area represents the cell morphology on the tissue culture plate $(\mathrm{MAG}=\times 150)$. 
cell death by biochemical and morphological events [Willingham 1999]. To determine whether the action of the $\mathrm{Ch} / \mathrm{EA}$ composite films induce apoptotic cell death, the apoptosis assays using Annexin V-PE and 7-AAD were performed to quantify the percentages of the apoptotic cells. Because the higher decrease in the cell viability within a short time period occurred in the chitosan film containing 0.5 and $1 \%(\mathrm{w} / \mathrm{v})$ of ellagic acid, in this study, the chitosan film containing $0.5 \%(\mathrm{w} / \mathrm{v})$ of ellagic acid $(\mathrm{Ch} / \mathrm{EA} 0.5)$ was chosen to compare with the chitosan film as well as the untreated culture plate control.

As shown in Table 3.2, the cells experienced apoptosis even on the cell culture plate (control group). However, a significantly greater percentage $(p<0.05)$ of the apoptotic cells on the $\mathrm{Ch} / \mathrm{EA} 0.5$ was observed when compared with the culture plate control and the chitosan film. Besides, most of the apoptotic cells induced by the $\mathrm{Ch} / \mathrm{EA} 0.5$ were populated in the early stage of apoptosis. This result was consistent with the result of the cell viability and proliferation at 24 hours of the incubation. Cell viability was significantly reduced by the treatment of the $\mathrm{Ch} / \mathrm{EA} 0.5$ through apoptotic mechanism within 1 day.

As shown in the surface morphological features (Figure 3.4), most of the melanoma cells cultured on the $\mathrm{Ch} / \mathrm{EA} 0.5$ undergoing the apoptotic process remained a round shaped and shrunk in their morphologies with time. In contrast, the morphological changes of the melanoma cells cultured on the chitosan film were found. This result demonstrated that apoptotic cell death via these therapeutic approaches would be expected to minimize inflammatory effects causing the damage in the surrounding tissue and provide highly effective strategies for cancer treatment with minimal side effects.

\subsection{Discussion}

In an attempt to apply a novel drug delivery system for cancer treatment, ellagic acid, a naturally occurring phenolic compound, was incorporated into the natural chitosan polymer. This study focused on development of chitosan/ellagic acid (Ch/EA) composite films and evaluation of their anti-cancer activities. The characteristics of the composite films were investigated regarding chemical structure, crystallinity, and surface morphology. The anti-cancer activities were compared according to different concentrations of ellagic acid and the localized effect was monitored via indirect cell culture and cellular morphological observation. The hypothesis was that the chitosan/ellagic acid composite films designed as a local drug delivery would present an anti-proliferative effect against the melanoma cells by inducing apoptosis.

Surface chemistry, crystallinity, and morphology of materials significantly influence the function of drug delivery system. The FTIR spectra revealed higher intensity peaks of amide and ester linkages in the $\mathrm{Ch} / \mathrm{EA}$ composite films compared with the chitosan film. This is probably because the carboxylic groups $(\mathrm{COOH})$ of the ellagic acid combined with the hydroxyl $(\mathrm{OH})$ and amine $\left(\mathrm{NH}_{2}\right)$ groups of the chitosan, resulting in increase in the amide and ester linkages [Tien 2003, Wang 2004]. 
Table 3.2 Induction of apoptosis in melanoma cells cultured on the indicated samples for 2 and 24 hours.

\begin{tabular}{ccccc}
\hline \multirow{2}{*}{ Apoptosis } & Control & Chitosan & Ch/EA0.5 \\
\hline \multirow{2}{*}{2 hours } & The early stage & $10.17 \pm 0.96$ & $8.84 \pm 0.99$ & $34.15 \pm 4.14 *$ \\
& The end stage & $0.22 \pm 0.06$ & $6.25 \pm 0.6$ & $0.46 \pm 0.03 * *$ \\
\hline \multirow{2}{*}{24 hours } & The early stage & $5.95 \pm 1.26$ & $9.43 \pm 1.03$ & $63.89 \pm 3.24 *$ \\
& The end stage & $6.32 \pm 1.04$ & $7.37 \pm 0.94$ & $3.16 \pm 0.24 * *$ \\
\hline
\end{tabular}

Cells were harvested at a density of $5 \times 10^{5}$ cells/well and analyzed apoptosis using Annexin V-PE and 7-AAD. Results are expressed as the percentage of apoptotic cells that were either Annexin V-positive and 7AAD-negative (apoptotic cells in the early stage) or Annexin V-positive and 7AAD-positive (apoptotic cells in the end stage or necrotic cells). * denotes significant difference compared with control and chitosan groups at both 2 and 24 hours incubation $(p<<0.05)$. ** denotes significant difference compared with the early stage at both 2 and 24 hours incubation $(p<<0.05)$. Each value represents the mean \pm SD ( $\mathrm{n}=3$ per each group). 
The surface of the composite films became rougher with increasing concentration of ellagic acid, thus indicating ellagic acid was physically entrapped into the chitosan polymer. The water contact angle of the $\mathrm{Ch} / \mathrm{EA}$ composite films decreased with increasing concentration of ellagic acid, suggesting enhanced wettability of the composite films. The increased wettability is probably due to increase in functional groups of ellagic acid on the composite films [Amaral 2006, Neamnark 2007] and surface roughness as shown in SEM (Figure 3.3). As such, this study suggested that the concentration of ellagic acid influenced surface functional groups, surface roughness, and wettability of the $\mathrm{Ch} / \mathrm{EA}$ composite film. Neutralized chitosan film showed a strong reflection of the hydrated crystal at $2 \theta$ of $10.5^{\circ}$ and $21^{\circ}$, and a characteristic broad halo, which is an indicator of the amorphous form of the chitosan films [Jaworska 2003, Silvia 2004, Vishu-Kumar 2004]. These peaks disappeared in the XRD patterns of the Ch/EA0.05 and $\mathrm{Ch} / \mathrm{EA} 0.1$ and were replaced by a broad peak, suggesting a lower crystallinity or a less ordered structure than the chitosan film. On the other hand, new diffraction peaks were observed in the $\mathrm{Ch} / \mathrm{EA} 0.5$ and $\mathrm{Ch} / \mathrm{EA} 1$, which were assigned to the crystalline of ellagic acid.

Cell adhesion, growth, and proliferation on the material surface are the sequential responses, which are essential for cell survival and function [Findlay 2000, Willingham 1999]. The morphological changes of the fibroblasts and melanoma cells cultured on the $\mathrm{Ch} / \mathrm{EA}$ composite films reflected a direct cell response to the biological activity of the composite films. The HS68 human fibroblasts changed their morphology from a circular shape to spindle-like features over time on the surface of the chitosan film, Ch/EA 0.05 , and $\mathrm{Ch} / \mathrm{EA}$ 0.1. In addition, cell viability was also significantly increased with time, indicating no harmful effect of the chitosan film and $\mathrm{Ch} / \mathrm{EA} 0.05$ on fibroblasts. In contrast, the WM115 human melanoma cells cultured on the same films initially attached and then detached and aggregated, showing initial inhibitory effect of films on cell attachment. This result demonstrated that the chitosan film had initial inhibitory effect on cell growth and proliferation for short time periods but it could not prevent cell proliferation for long time periods. Several studies have suggested that the chitosan induces anti-bacterial and anti-tumor properties attributed to the tumor necrosis factor- $\alpha$ (TNF- $\alpha$ ) in human monocytes [Muzzarelli 1997, Ravi Kumar 2006]. However, additional studies are needed to clarify the mechanism by which chitosan polymer influences the growth of cancer cells. However, with increasing concentration of ellagic acid ( 0.5 and $1 \%(\mathrm{w} / \mathrm{v}))$, both the fibroblasts and melanoma cells remained in the initial round shape and could not grow and proliferate. The number of viable cells was significantly reduced by treatment of the $\mathrm{Ch} / \mathrm{EA} 0.5$ and $\mathrm{Ch} / \mathrm{EA} 1$ even after 4 hours incubation. In other words, the cells initially adhered to the composite films, but they lost their viability within a few hours in an ellagic acid concentration dependent manner.

In addition, this study showed the biological activity of the Ch/EA composite films is localized. It was clearly supported by the cell behavior at the interface of the $\mathrm{Ch} / \mathrm{EA} 1$ for 3 days incubation as shown in Figure 3.8. At the interface, both melanoma and fibroblasts could grow and proliferate well in the absence of direct contact on the $\mathrm{Ch} / \mathrm{EA} 1$. However, cells lost their viability in the presence of direct contact on the $\mathrm{Ch} / \mathrm{EA} 1$, and they remained a round shaped and shrunk in their morphologies with time. This result demonstrated that the $\mathrm{Ch} / \mathrm{EA}$ composite films influence cell survival and 
growth mostly via direct contact, corresponding with slow release of ellagic acid from chitosan matrix into cell culture media. Additionally, the indirect contact culture showed the Ch/EA1 significantly slowed down the proliferation of melanoma cells compared with chitosan film. This result suggested that the chitosan film containing higher concentration of ellagic acid was able to regulate the cell growth via the released ellagic acid from chitosan film into culture medium. This study verified the localized and dosedependent effect of composite films on melanoma, which potentially made it possible to apply the composite films to treat cancers at targeted area.

The $\mathrm{Ch} / \mathrm{EA}$ composite film could induce apoptotic cell death in a dose-dependent manner. Induction of apoptosis has been considered as a target of anti-cancer therapy. Apoptosis is a process of an essential tissue homeostasis, thus being regarded as an ideal way to treat cancer without significant influence on normal tissue [Bai 2006, Khan 2007]. Many studies have demonstrated that induction of apoptosis by anti-cancer drugs appeared in a dose- and time-dependent manner. [Khan 2007, Losso 2004, Narayanan 1999] This study has an agreement with the fact that the $\mathrm{Ch} / \mathrm{EA}$ composite films induce apoptotic cell death in an ellagic acid concentration dependent manner. In the present study, it has been discussed that a localized activity of the $\mathrm{Ch} / \mathrm{EA}$ composite films is attributed to the unique characteristics of chitosan and ellagic acid. This novel approach showed a new clinical approach to treat cancer at a targeted site by induction of apoptosis, thus having certain advantages over conventional cancer chemotherapy.

\subsection{Conclusion}

This study was to investigate the feasibility of a local delivery of ellagic acid using chitosan polymer for cancer treatment. In summary, ellagic acid was incorporated into the chitosan polymer chemically and physically. The concentration of ellagic acid influenced chemical structure, surface morphology, hydrophilicity, and crystallinity of the composite film. Additionally, the composite films were found to have a potent anti-cancer activity against melanoma cells in an ellagic acid concentration dependent manner and induce apoptotic cell death. It was concluded from this study that the Ch/EA composite film represents a new form of ellagic acid for a local cancer treatment. 


\title{
CHAPTER 4. THE INHIBITION OF GLIOMA GROWTH IN VITRO AND IN VIVO BY A CHITOSAN/ELLAGIC ACID COMPOSITE BIOMATERIAL*
}

\begin{abstract}
4.1 Summary
This study investigated the anti-cancer effect of a local delivery of ellagic acid against brain cancer. The Ch/EA composite films were fabricated according to different concentrations of ellagic acid. In vitro release study was performed by a UV spectrophotometer, and enzymatic degradation rate was determined by analyzing the increase in free amino groups. Viability of cancer cells (human U87 glioblastomas and rat C6 glioma cells) was measured via direct and indirect cell culture on the films by a MTS assay. To analyze anti-cancer mechanisms, caspase-3 activation, western blot for $\mathrm{p} 53$, and anti-angiogenesis assays were examined. In the in vivo study, GFP-tagged rat C6 glioma cells were implanted subcutaneously at the right flank region of nude mice, and treatments were initiated by implanting the films subcutaneously. Tumor growth was evaluated by measuring tumor volume using a caliper, an ultrasound machine, and an optical imaging system. The $\mathrm{Ch} / \mathrm{EA}$ composite films were enzymatically degradable, indicated by at least a 5 times higher concentration of free amino groups in the incubation medium at 3 weeks compared with 1 day. They also exhibited a sustained slow release of ellagic acid without an initial burst effect. Tumor tissue growth in Ch/EA20 group was 9 times lower than that in control groups at 3 weeks observation by measuring a caliper. It was concluded that the $\mathrm{Ch} / \mathrm{EA}$ composite film can inhibit the growth of brain cancer cells in an ellagic acid concentration-dependent manner by inducing apoptosis of cancer cells as well as suppressing angiogenesis.
\end{abstract}

\subsection{Introduction}

Primary brain tumors mostly arise from glial cells of the brain itself while secondary brain tumors are originated from the body and spread to the brain [Brandes 2008, Cokgor 1998]. Abnormal growth of glial cells is responsible for the development of gliomas. The glioma includes any type of brain tumor such as astrocytoma, oligodendroglioma, and glioblastoma with a different function [Brandes 2008]. Glioma is classified into four grades according to its appearance under the microscope. Low-grade gliomas (grade I and II) are slow-growing and curable while high-grade gliomas (grade III and IV) are fast-growing, invasive, and frequently recur [Graham 2004]. Glioblastoma multiforme (GBM) is the most malignant brain tumor, infiltrating adjacent normal brain tissue. GBM spreads rapidly, invades other parts of the brain, and mostly recur after initial treatment [Brandes 2008]. It is a grade IV tumor which includes increase in cell population, abnormal cells and nuclei, necrosis and growth of blood vessels [Purow 2004].

* Adapted with permission of Elsevier. Kim S, Gaber MW, Zawaski JA, Zhang F, Richardson M, Zhang XA, Yang Y. The inhibition of glioma growth in vitro and in vivo by a chitosan/ellagic acid composite biomaterial. Biomaterials 2009;30:4743-4751. 
It was reported that the median survival time of patients with malignant gliomas ranges from 14 weeks (no treatment) to 40-50 weeks despite aggressive multimodality management with surgery, radiation, and chemotherapy [Avgeropoulos 1999, Graham 2004]. This is because high grade gliomas mostly recur locally. In this regard, local therapies combined with surgical intervention are regard as an ideal approach to improve the efficacy of treatments. Recent studies have focused on designing polymeric devices that allow local delivery of chemotherapeutics into the surgical resection cavity. In particular, for patients with brain cancer, these local delivery systems are safer and more beneficial compared with traditional chemotherapy [Avgeropoulos 1999, Moses 2003, Wang 2002]. These methods administer drugs directly into the brain, bypass the blood brain barrier (BBB), and deliver the drugs in a concentration dependent manner [Groothuis 2000, Purow 2004, Wang 2002]. Some clinical studies have demonstrated that local chemotherapy delivered by polymer carrier significantly prolongs the survival time of patients [Brem 1999, Cho 2008, Jain 2001, Walter 1994, Westphal 2003].

It is generally agreed that ellagic acid inhibits growth of cancer cells via induction of apoptosis or by suppressing angiogenesis. Ellagic acid induces G1 arrest and apoptosis by p53-mediated activation [Khan 2007, Mertens-Talcott 2005]. Unlike necrosis, apoptosis can reduce inflammatory response caused by cell death. Because the apoptotic function of p53 is critical for tumor suppression, induction of apoptosis through p53 activation may be an attractive strategy for anti-cancer treatment. Ellagic acid also inhibits angiogenesis via inactivating metalloproteinases by chelating divalent cations such as $\mathrm{Zn}^{2+}$ and $\mathrm{Cu}^{2+}$ [Khan 2007, Labrecque 2005, Laura 2001, Oak 2005]. As a result, tumor growth and formation of new blood vessels are possibly suppressed by lowering the level of such cations. The angiogenesis is an important process for tumor growth and metastasis. Therefore, controlling the actions of the cellular and molecular levels involved in angiogenesis provide novel therapeutic approaches for cancer treatment. Ellagic acid is currently used as a dietary supplement to reduce or prevent the risk of cancer. [Pinto 2008, Vekiari 2008]

However, most dietary phenolic products are poorly absorbed into the body, rapidly metabolized, and eliminated by digestive and hepatic activities or the bioactivities of phenolic metabolites in blood [Han 2007, Scalbert 2000]. This result leads to the absence of ellagic acid in blood or presence in very low concentrations [Shukla 2008]. In order to increase the bioavailability and maximize the anti-tumor activity, a local delivery system using a chitosan polymer has been developed and attempted to carry ellagic acid over a long period of time to treat brain cancers. Chitosan, a cationic natural biopolymer, can be readily formed into film-type or gel-type and is promising in the development of controlled-release systems [Kim 2009, Ravi Kumar 2004, Tien 2003]. It has been extensively used as a drug carrier, a wound healing agent, and for tissue regeneration in a variety of applications. The objective of this study was to further study release profiles, degradation behaviors, and to evaluate its biological effect and mechanism against brain cancers (human U87 glioblastomas and rat C6 glioma cells) in vitro and in vivo. It was hypothesized that $\mathrm{Ch} / \mathrm{EA}$ composite films inhibit growth of glioma cancer by inducing apoptosis and suppressing angiogenesis. 


\subsection{Materials and Methods}

\subsubsection{Materials}

The chitosan with approximately $85 \%$ of a degree of deacetylation and ellagic acid were purchased from Sigma Aldrich. Fetal bovine serum (FBS), trypsin/EDTA, Lglutamine, antibiotic/antimycotic, phosphate buffered saline (PBS), and Dulbecco's Modified Eagle Medium (DMEM) were obtained from Gibco ${ }^{\mathrm{TM}}$. Lysozyme (egg-white), glycine (crystalline/certified), and ninhydrin solution reagents were purchased from Fisher Scientific. The U87 human glioblastoma and C6 rat glioma cell lines were cultured as per American Type Culture Collection (ATCC) instructions. The Human Umbilical Vein Endothelial Cells (HUVEC) was purchased from and cultured as per Cascade Biologics $^{\mathrm{TM}}$ (Portland, OR, USA).

\subsubsection{Preparation of Chitosan Films (Ch)}

A $1 \%(\mathrm{w} / \mathrm{v})$ chitosan solution was prepared by stirring powdered chitosan in $0.75 \%(\mathrm{v} / \mathrm{v})$ aqueous acetic acid at room temperature overnight. It was followed by dialysis for 6 days using a dialysis membrane, and its final $\mathrm{pH}$ value was approximately 6.6. The neutralized chitosan solution was autoclaved $\left(120^{\circ} \mathrm{C}, 20 \mathrm{~min}\right)$, and a chitosan film was prepared by evaporation for 1 day. The dry transparent film was washed with distilled water to remove salt and then air dried in a biological hood.

\subsubsection{Preparation of Chitosan/Ellagic Acid (Ch/EA) Composite Films}

Ellagic acid solutions were prepared according to different concentrations $(0.05$, $0.1,0.5,1$, or $20 \%(\mathrm{w} / \mathrm{v}))$ of ellagic acid powder. The ellagic acid was dissolved into distilled water by adding several drops of $0.1 \mathrm{M} \mathrm{NaOH}$, with constant stirring at room temperature overnight. The chitosan solutions were then mixed with various ellagic acid solutions at a chitosan to ellagic acid volume ratio of $1: 1$. The mixed $\mathrm{Ch} / \mathrm{EA}$ solutions were agitated at $40^{\circ} \mathrm{C}$ for 2 hours and were then neutralized for 3 or 4 days using dialysis membranes to reach the final $\mathrm{pH}$ values, ranging from 6.1 to 6.6. The $\mathrm{Ch} / \mathrm{EA}$ solutions were autoclaved $\left(120^{\circ} \mathrm{C}, 20 \mathrm{~min}\right)$ and cast in non-stick plates and dried by evaporation for 1 day in the biological hood. Chitosan/ellagic acid composite films (Ch/EA0.05, $\mathrm{Ch} / \mathrm{EA} 0.1, \mathrm{Ch} / \mathrm{EA} 0.5, \mathrm{Ch} / \mathrm{EA} 1$, and $\mathrm{Ch} / \mathrm{EA} 20)$ were carefully peeled off from the plates, washed with distilled water to remove salt, and then air-dried in a biological hood.

\subsubsection{In vitro Release Studies}

Ellagic acid release from the $\mathrm{Ch} / \mathrm{EA}$ composite films was quantified over time by an in vitro release assay. Chitosan/ellagic acid composite films (Ch/EA0.05, Ch/EA0.1, $\mathrm{Ch} / \mathrm{EA} 0.5, \mathrm{Ch} / \mathrm{EA} 1$, or $\mathrm{Ch} / \mathrm{EA} 20)$ were prepared from the same volume of each polymer 
solution, which contains the same weight percentage of chitosan but different weight percentages of ellagic acid. The films were then cut and placed in containers containing 6 $\mathrm{mL}$ of distilled water at $37^{\circ} \mathrm{C}$. At designated time points $(1,3,7,14$, and 21 days $), 500 \mu \mathrm{L}$ aliquots of the release medium were sampled and the same amount of fresh distilled water was added into the containers. In the collected fractions, the cumulative amount of ellagic acid released from the composite films was determined as a function of time by a UV spectrophotometer (ULTRASPEC $2100 \mathrm{PRO}$ UV/Visible) at $260 \mathrm{~nm}$. In addition, ellagic acid release in the presence of lysozyme $(1 \mathrm{mg} / \mathrm{mL})$ was also determined in order to examine the effect of an enzyme on the release profile.

\subsubsection{In vitro Enzymatic Degradation}

The chitosan/ellagic acid composite films (chitosan, Ch/EA0.05, Ch/EA0.1, $\mathrm{Ch} / \mathrm{EA} 0.5, \mathrm{Ch} / \mathrm{EA} 1$, or $\mathrm{Ch} / \mathrm{EA} 20)$ were prepared after sterilization by autoclaving $\left(120^{\circ} \mathrm{C}\right.$, $20 \mathrm{~min}$ ) and then digested in 24-well plates containing phosphate buffer saline (PBS, pH 6) with $1 \mathrm{mg} / \mathrm{mL}$ lysozyme (egg-white, Fisher Scientific, Fair Lawn, NJ) at $37^{\circ} \mathrm{C}$. In this study, the ninhydrin assay was performed to determine free amino groups in the incubation medium collected after the designated time points.

Two separate studies were performed to determine the degradation rates of $\mathrm{Ch} / \mathrm{EA}$ composite films and the effect of the ratio of chitosan to ellagic acid on enzymatic degradation. The first study was to evaluate the effect of lysozyme on degradation of each sample with the same total weight (approximately $12 \mathrm{mg}$ ). The second study was to investigate the degradation rate of each sample with the same weight of chitosan but different weight percentages of ellagic acid. After the predetermined time points $(1,3,7$, 14, and 21 days), $0.5 \mathrm{~mL}$ of the medium from each sample was collected and heated with $0.5 \mathrm{~mL}$ of ninhydrin solution at $95^{\circ} \mathrm{C}$ for $10 \mathrm{~min}$. After heating with ninhydrin solution, the light absorbance at $550 \mathrm{~nm}$ was recorded using a micro-plate reader (Molecular Device Vmax kinetic). The glycine (Fisher Scientific, Fair Lawn, NJ) was used as an amino acid nitrogen standard at various known concentrations. Because the amount of free amino groups is proportional to the optical absorbance of the solution, the enzymatic degradation rate can be determined by analyzing the increased $\mathrm{N}$-glucosamine units in the incubation medium.

\subsubsection{Cell Culture}

The U87 human glioblastomas were grown and maintained in DMEM media with $10 \%$ FBS, $1 \%$ antibiotic/antimycotic mixture, $5 \mathrm{~mL}$ of L-glutamine (200 $\mathrm{mM})$, and sodium pyruvate. The $\mathrm{C} 6$ rat gliomas were grown and maintained in F-12K Media with $2.5 \%$ FBS, $15 \%$ horse serum, $1 \%$ antibiotic/antimycotic mixture, $5 \mathrm{~mL}$ of L-glutamine (200 mM), and sodium pyruvate. The Human Umbilical Vein Endothelial Cells (HUVEC) were grown and maintained in Medium 200 with Low Serum Growth Supplement (LSGS) containing fetal bovine serum $(2 \% \mathrm{v} / \mathrm{v})$, hydrocortisone $(1 \mathrm{mg} / \mathrm{mL})$, human epidermal growth factor $(10 \mathrm{ng} / \mathrm{mL})$, basic fibroblast growth factor $(3 \mathrm{ng} / \mathrm{mL})$, 
and heparin $(10 \mathrm{mg} / \mathrm{mL})$. The cells were cultured in an incubator supplied with $5 \% \mathrm{CO}_{2}$ at $37^{\circ} \mathrm{C}$. The culture medium was changed every 3 days.

\subsubsection{In vitro Anti-Tumor Activity - Direct Cell Culture}

To determine the biological effect of $\mathrm{Ch} / \mathrm{EA}$ composite films on the two brain cancer cell lines, the numbers of viable cells (U87 and C6) were measured by a MTS assay. $150 \mu \mathrm{L}$ of the sterilized samples was coated onto each well of 48 -well plates and dried at room temperature followed by rinsing with PBS $(\mathrm{pH} 7.4)$ twice and then dried again. The cells were seeded on each sample at a density of 30,000 cells per well and cultured without medium exchange for 4, 24, and 72 hours. At the end of the incubation period, the cell viability was determined with the Cell Titer 96AQueous One Solution Cell Proliferation Assay (MTS assay, Promega Corp.). Each $60 \mu \mathrm{L}$ of MTS solution was added into each well of the 48 -well plate, containing the samples in $300 \mu \mathrm{L}$ of culture medium. Following an incubation period of 2 hours with $5 \% \mathrm{CO}_{2}$ at $37^{\circ} \mathrm{C}, 100 \mu \mathrm{L}$ of solution in each well of the 48-well plate was transferred into the 96 -well assay plates and the light absorbance at $490 \mathrm{~nm}$ was recorded using a micro-plate reader (Molecular Device Vmax kinetic). The background absorbance at $490 \mathrm{~nm}$ was corrected by subtracting the average absorbance of the control wells without cells from each well to estimate viable cellular activity.

\subsubsection{In vitro Anti-Tumor Activity - Indirect Cell Culture}

The effect of the Ch/EA composite films on cell viability was investigated via indirect cell culture using BD BioCoat ${ }^{\mathrm{TM}}$ Control Cell Culture inserts (12-well formats). This experiment was to evaluate cell response to the composite films through cell culture media. The insert contains a $0.45 \mu \mathrm{m}$ pore size PET membrane which allows the culture medium only permeable to the membrane. This experimental design is to observe cell response to the released ellagic acid from the composite films into cell culture media. The bottom of each well plate was coated with $700 \mu \mathrm{L}$ of a chitosan solution or a chitosan solution containing $1 \%(\mathrm{w} / \mathrm{v})$ of ellagic acid $(\mathrm{Ch} / \mathrm{EA} 1)$ and dried at room temperature followed by rinsing with PBS ( $\mathrm{pH} 7.4$ ) twice and then dried again. $1.7 \mathrm{~mL}$ of the cell culture medium was added into each well of the 12-well plate with inserts, which were allowed to hydrate for 2 hours at $37^{\circ} \mathrm{C}$. The cancer cells were then seeded at a density of 60,000 cells per well on the upper chamber with $0.3 \mathrm{~mL}$ of culture medium. After the incubation for 4, 24, and 72 hours, the number of viable cells was determined using the Cell Titer 96AQueous One Solution according to the manufacturer's instructions.

\subsubsection{Western Blot}

To determine whether the levels of p53 had changed after treatment, rat C6 glioma cells were treated with the $\mathrm{Ch} / \mathrm{EA} 1$ for western blot analysis. This study was to investigate the expression of apoptosis-related proteins in brain cancer cells. After cell collection, treated or untreated cells were lysed with $80 \mu \mathrm{L}$ of lysis buffer $(1 \%$ Triton X100) on ice and then $4 \mu \mathrm{L}$ of protease inhibitors were added to lysis buffer for $15 \mathrm{~min}$. 
Cell lysates were clarified by centrifugation at 13,200 rpm for $5 \mathrm{~min}$. The supernatant was transferred to a new tube and the remaining pellets were discarded. The samples were mixed with $25 \mu \mathrm{L}$ of a loading buffer (4X Laemmli buffer) to denature proteins and boiled at $90^{\circ} \mathrm{C}$ for $5 \mathrm{~min}$. To separate proteins according to their molecular weights, molecular weight markers as a standard ( $4 \mu \mathrm{L}$ per mini-gel well) and equivalent amounts of samples were loaded ( $40 \mu \mathrm{L}$ per mini-gel well) into the bottom of each well of a gel (10\% SDS-PAGE) with a running buffer (a standard migration buffer) by a micro-pipetter fitted with a long narrow tip.

Electrophoresis was started at $80 \mathrm{~V}$ for $90 \mathrm{~min}$ and then at $100 \mathrm{~V}$ for 1 hour. When the dye molecule reaches the bottom of the gel, the power was turned off. To transfer protein from the gel to a membrane in an electrical field, the membrane was placed next to the gel. The two were sandwiched between absorbent materials, and the sandwich was clamped between solid supports to maintain tight contact between the gel and membrane. The sandwich was submerged in a transfer buffer to which an electrical field $(40 \mathrm{~V})$ was applied overnight. The negatively-charged proteins traveled towards the positively-charged electrode, but the membrane stopped them. The membrane was incubated in a blocking solution which contains $5 \%$ milk in $100 \mathrm{~mL}$ of $0.05 \%$ PBST by shaking at room temperature for $30 \mathrm{~min}$. The membrane was then incubated with a primary antibody which was p53 mouse mAb (Cell Signaling Technology, 1:500 dilution) in a blocking solution at $4^{\circ} \mathrm{C}$ overnight. Integrin $\beta-1$ was used as an internal control. The membrane was washed with $0.05 \%$ PBST 3 times and then incubated with a secondary antibody which was HRP conjugated anti-mouse IgG at a dilution of 1:40,000 for p53 or Integrin $\beta-1$ at room temperature for $30 \mathrm{~min}$. The membrane was then washed with $0.05 \%$ PBST 3 times, after which proteins were detected using enhanced luminal reagents for 1 $\min$.

\subsubsection{Caspase-3 Activation}

In order to investigate apoptotic cell death in response to $\mathrm{Ch} / \mathrm{EA}$ composite films, caspase-3 activity was measured by ApoAlert caspase-3 assay kit from Clontech (Palo Alto, CA). Several studies have shown that caspase-3 activity is increased through a protease cascade during apoptosis in the early stage. Following treatment with the chitosan film for 6 hours and the Ch/EA1 for 2 hours or 6 hours, cells were trypsinized, washed twice in PBS, and then aliquoted at $2.5 \times 10^{6}$ cells per test, after which samples were centrifuged at $1,450 \mathrm{rpm}$ for $5 \mathrm{~min}$. After resuspending in $50 \mu \mathrm{L}$ of chilled cell lysis buffer, cells were incubated on ice for $10 \mathrm{~min}$. Cell lysates were centrifuged in a microcentrifuge at maximum speed $(66,000 \mathrm{rpm})$ for $10 \mathrm{~min}$ to precipitate cellular debris, and then the supernatants were transferred into new microcentrifuge tubes. $50 \mu \mathrm{L}$ of $2 \mathrm{X}$ reaction buffer containing DTT (1:100 dilution) was added to each supernatant, after which $5 \mu \mathrm{L}$ of caspase-3 substrate (DEVD-p-nitroanilide, $1 \mathrm{mM}$ ) was added to each tube. Samples were incubated at $37^{\circ} \mathrm{C}$ for 1 hour and transferred to a 96 -well plate for a platereading in a micro-plate reader at $405 \mathrm{~nm}$ (Molecular Device Vmax kinetic) according to the manufacturer's instructions provided with the assay kit. 


\subsubsection{In vitro Angiogenesis Assay}

To evaluate the ability of endothelial cells to form tube-like structures after treatment, in vitro angiogenesis assay was performed with a gel of basement membrane (Chemicon International, Inc., Temecula, CA). A gel matrix consisted of laminin, collagen type IV, heparan sulfate, proteoglycans, and entactin. It also contained various growth factors (TGF- $\beta$, FGF) and proteolytic enzymes (plasminogen, tPA, MMPs) for maximal tube-formation. This assay was used to monitor the extent of tube assembly in human umbilical vein cells (HUVEC). To prepare the gel matrix, $100 \mu \mathrm{L}$ of $10 \mathrm{X}$ diluent buffer was added to $900 \mu \mathrm{L}$ of ECMatrix ${ }^{\mathrm{TM}}$ solution in a sterile microfuge tube on ice to avoid solidification. After preparing the gel matrix, $50 \mu \mathrm{L}$ of solution was transferred to each pre-cooled 96-well tissue culture plate and incubated at 37C for one hour to allow the matrix solution to solidify. The wells were then incubated for 8 hours at ${ }^{\circ} 37$ with HUVEC cells $\left(1 \times 10^{4}\right.$ cells/well). The HUVEC were pre-cultured in the absence (control) or presence of the films (chitosan film or Ch/EA1) for 1 day before they were transferred into the gel matrix. The tube formation was examined microscopically using an inverted bright field (BF) microscope (Nikon ECLIPSE TE-2000U) and the images (100 X) were processed using MetaVue software.

\subsubsection{In vivo Anti-Tumor Activity}

Female nude mice, 8-10 weeks of age and weighing $24 \mathrm{~g}$ to $30 \mathrm{~g}$, were obtained from Charles River Laboratories, Inc. (Wilmington, MA, USA). A total of 15 female nude mice were used to evaluate the anti-tumor activity and toxicity of $\mathrm{Ch} / \mathrm{EA}$ composite films, with five mice per group. All mice were housed in standard facilities with not more than three mice per cage and were given free access to water and food before treatments were initiated. Each mouse was inoculated subcutaneously with $4 \times 10^{6}$ cells in $200 \mu 1$ of PBS of GFP (green fluorescent protein) tagged C6 rat glioma in the right flank. Each animal was weighed on the day of tumor injection. Each mouse was imaged and its tumor volume was measured using a caliper, an ultrasound machine (Philips HDI 5000), and Fluorescence Reflectance Imaging (FRI) system. Animals were anesthetized with an isoflurane/oxygen inhalation mixture during imaging procedures. Tumor volume was calculated according to the following formula: tumor volume $\left(\mathrm{mm}^{3}\right)=$ (major axis) $\times$ (minor axis) ${ }^{2} \times 0.52$ [Li 2004]. The major axis is the longest diameter of the tumor and the minor axis is the maximal line drawn perpendicular to the major axis [Kim 2007, Schwartz 2000, 2006].

Treatment was initiated by implanting either a chitosan film or $\mathrm{Ch} / \mathrm{EA} 20$ film subcutaneously on the fifth day after tumor inoculation. In this study, higher concentration of ellagic acid $(20 \%(\mathrm{w} / \mathrm{v}))$ was employed to effectively treat a tumor tissue because anti-tumor effect of the composite film depended on concentration of ellagic acid. The tumor was allowed to establish until the area was approximately 50-90 $\mathrm{mm}^{2}$ on day 5 post implantation as measured by the product of two orthogonal diameters using a caliper. At this point, the tumors were generally small and visually detectable. This time point was chosen in order to treat the primary tumors at an early stage as well 
as mimic treatment of the tumor residues following surgical resection. For the tumor control group, tumors were left untreated. For the two surgical intervention groups, after an incision was made around a tumor site with a scalpel, a chitosan film or a $\mathrm{Ch} / \mathrm{EA} 20$ was inserted beneath the tumor site and then the skin was sutured. After the treatments, all mice were caged individually under veterinary care and supervision. Animals were weighed and imaged every 2 days for signs of tumor growth and sacrificed if their body weight decreased by $10 \%$ of the starting weight or if the tumor size reached $2 \mathrm{~cm}$ in a diameter. Experimental data were expressed as the tumor volume change for anti-tumor effect of treatment and weight loss for toxicity.

The histology study was performed to examine the tumor cell population and the acute toxicity at the implantation site. Animals were euthanized after 21 days of observation and then tumors were retrieved along with adjacent tissue and fixed in $10 \%$ neutral buffered formalin before histology analysis. After fixation, tissue samples were embedded in paraffin. The paraffin embedded tissues were sectioned, deparaffinized with xylene, and then stained with hematoxylin and eosin for microscopic analysis. All of the animal experiments were approved by the Animal Welfare and Ethics Committee of the University of Tennessee Health Science Center.

\subsubsection{Statistical Analysis}

All data are presented as mean \pm standard deviation. Significant differences in release profile, degradation rate, cell activity, and tumor volume were analyzed by oneway ANOVA test. The differences in groups and experimental time points were considered significant if $p<0.05$.

\subsection{Results}

\subsubsection{In vitro Release Studies}

In order to investigate the ellagic acid release from the composite films under in vitro conditions, the cumulative amount of ellagic acid in the medium was measured as a function of time. Figure 4.1 shows the resulting release profiles of the ellagic acid from the composite films. As shown in Figure 4.1a, the initial drug loading did not affect the release rate for 2 weeks, but significant difference was observed between the films containing the higher ellagic acid loading (Ch/EA20) and lower ellagic acid loadings $(\mathrm{Ch} / \mathrm{EA} 0.05, \mathrm{Ch} / \mathrm{EA} 0.1$ and $\mathrm{Ch} / \mathrm{EA} 0.5)$ at the 3 week incubation periods $(p<0.05)$. It was also observed that the $\mathrm{Ch} / \mathrm{EA}$ composite films exhibited a sustained slow release of ellagic acid.

In addition, another release profile of ellagic acid was performed in the presence of lysozyme to evaluate the effect of lysozyme on the release rate of ellagic acid. As shown in Figure 4.1b, the cumulative amount of the ellagic acid released from the films 


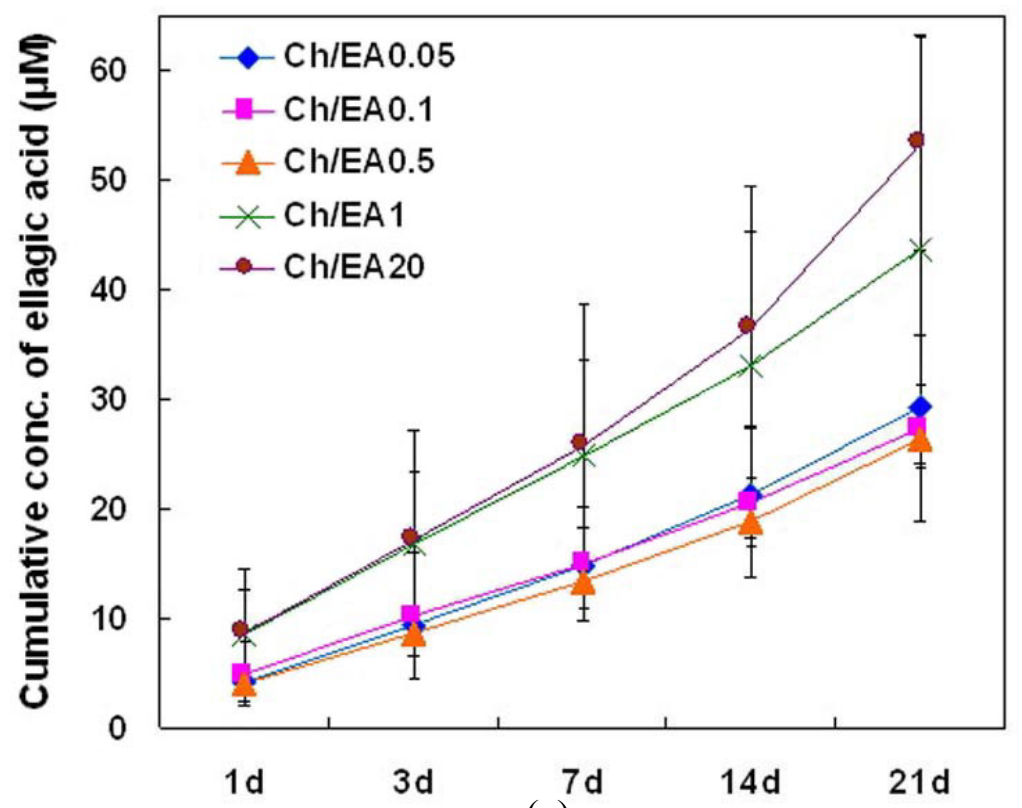

(a)

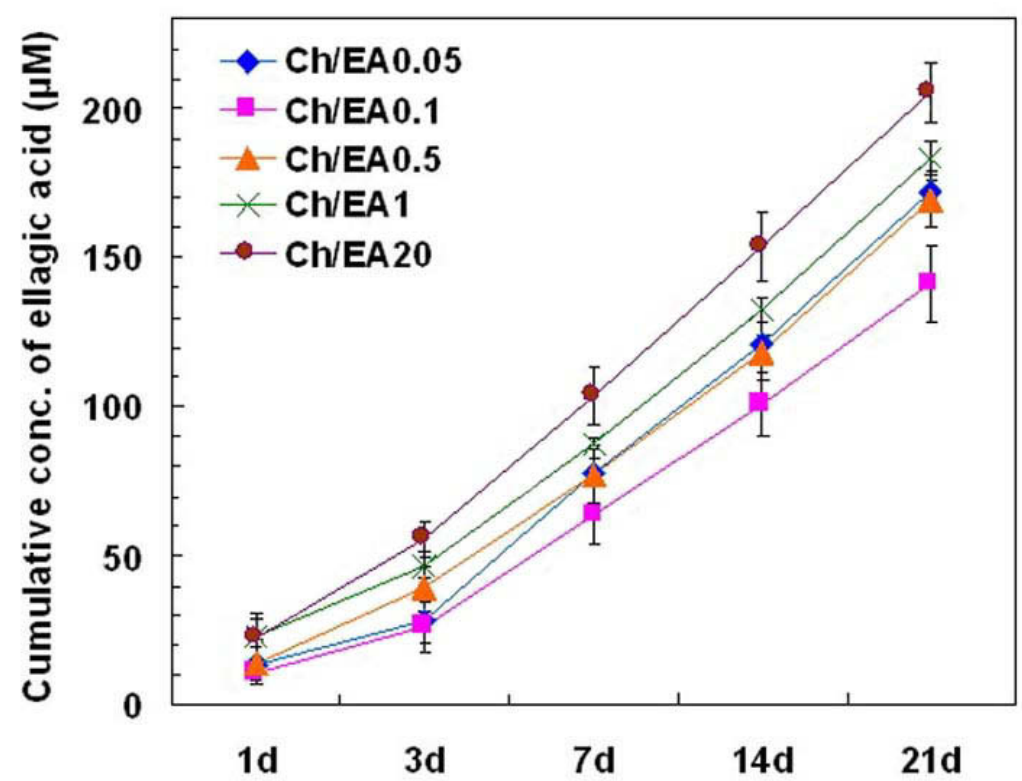

(b)

Figure 4.1 In vitro cumulative amount of the ellagic acid released from the Ch/EA composite films. (a) absence of lysozyme and (b) presence of lysozyme (a and b are different in scale). The cumulative amount of the released ellagic acid was determined as a function of time by a UV spectrophotometer at $260 \mathrm{~nm}$. Each value represents the mean $\pm \mathrm{SD}$ ( $\mathrm{n}=3$ per each group). 
in the presence of lysozymes was significantly higher than the absence of lysozymes. There was a significant difference between the Ch/EA20 and the others after 1-week incubation due to initial loading difference $(p<0.05)$. This result demonstrated that the presence of lysozyme could increase the release of the ellagic acid from the $\mathrm{Ch} / \mathrm{EA}$ composite films. It is worth noting that both release profiles of the ellagic acid from the films in the absence and presence of lysozyme did not show an initial burst effect but exhibited a sustained slow release.

\subsubsection{In vitro Enzymatic Degradation}

To determine the degradation behavior of the $\mathrm{Ch} / \mathrm{EA}$ composite films, the amounts of enzymatic-hydrolyzed films were measured by ninhydrin assay. Lysozyme can interact with chitosan films in an acidic condition around $\mathrm{pH}$ 5-6 and induce enzymatic degradation of samples. Figure 4.2a shows the degradation rates of the $\mathrm{Ch} / \mathrm{EA}$ composite films with the same total weight for 3 weeks in lysozyme medium. The results showed significant increases in free amino groups corresponding to N-glucosamine from all the groups after 3 weeks incubation compared with 1 day incubation $(p<0.05)$. This result indicates a continuous degradation of the films within the experimental period. In addition, the films containing lower concentration of ellagic acid $(\mathrm{Ch}, \mathrm{Ch} / \mathrm{EA} 0.05$, and $\mathrm{Ch} / \mathrm{EA} 0.1)$ exhibited significantly $(p<0.05)$ higher enzymatic-degradation rates than the chitosan films containing higher concentration of ellagic acid $(\mathrm{Ch} / \mathrm{EA} 0.5, \mathrm{Ch} / \mathrm{EA} 1$, and $\mathrm{Ch} / \mathrm{EA} 20)$. This result demonstrated that the composite films containing higher weight percentage of chitosan released more free amino groups into the medium, indicating higher degradation rate.

Figure $4.2 \mathrm{~b}$ shows the degradation rates of the $\mathrm{Ch} / \mathrm{EA}$ composite films containing the same weight percentage of chitosan but different weight percentage of ellagic acid. There were significant increases in free amino groups from all the composite films after 3 weeks incubation compared with 1 day incubation $(p<0.05)$. This result also demonstrated that different concentrations of ellagic acid incorporated into chitosan films influenced degradation rates of the composite films even though the weight percentage of chitosan was same in all groups. The composite films containing higher concentration of ellagic acid (Ch/EA0.5, $\mathrm{Ch} / \mathrm{EA} 1$, and $\mathrm{Ch} / \mathrm{EA} 20)$ exhibited lower degradation rates compared with lower concentration of ellagic acid (Chitosan, $\mathrm{Ch} / \mathrm{EA} 0.05$, and $\mathrm{Ch} / \mathrm{EA} 0.1$ ). This result suggested that the degradation rate of the $\mathrm{Ch} / \mathrm{EA}$ composite films was affected by the weight percentage of chitosan and concentration of ellagic acid. This study showed that the Ch/EA composite films are enzymatically biodegradable, corresponding to interactions between chitosan and ellagic acid.

\subsubsection{Cell Viability - Direct Contact}

Anti-proliferative effect of the $\mathrm{Ch} / \mathrm{EA}$ composite films on human U87 glioblastoma and rat C6 glioma cells was investigated by MTS assay. As shown in Figure 4.3, there were significant increases in viability of U87 glioblastoma and C6 glioma cells 


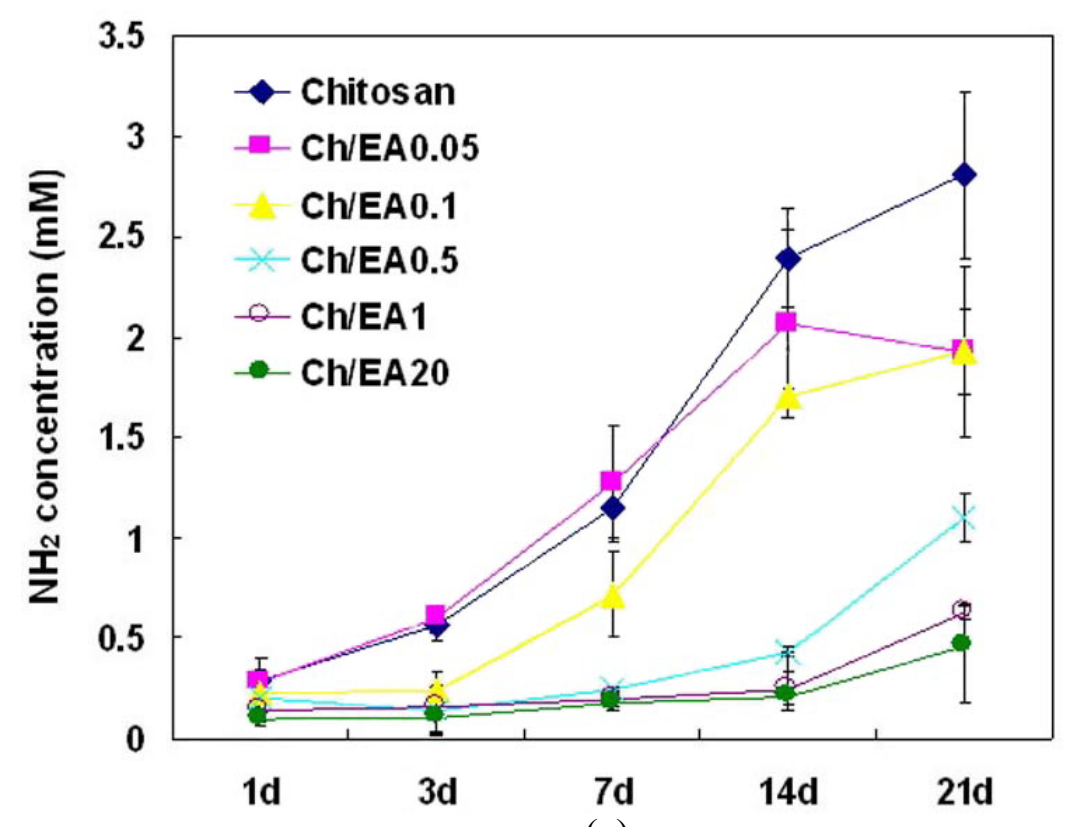

(a)

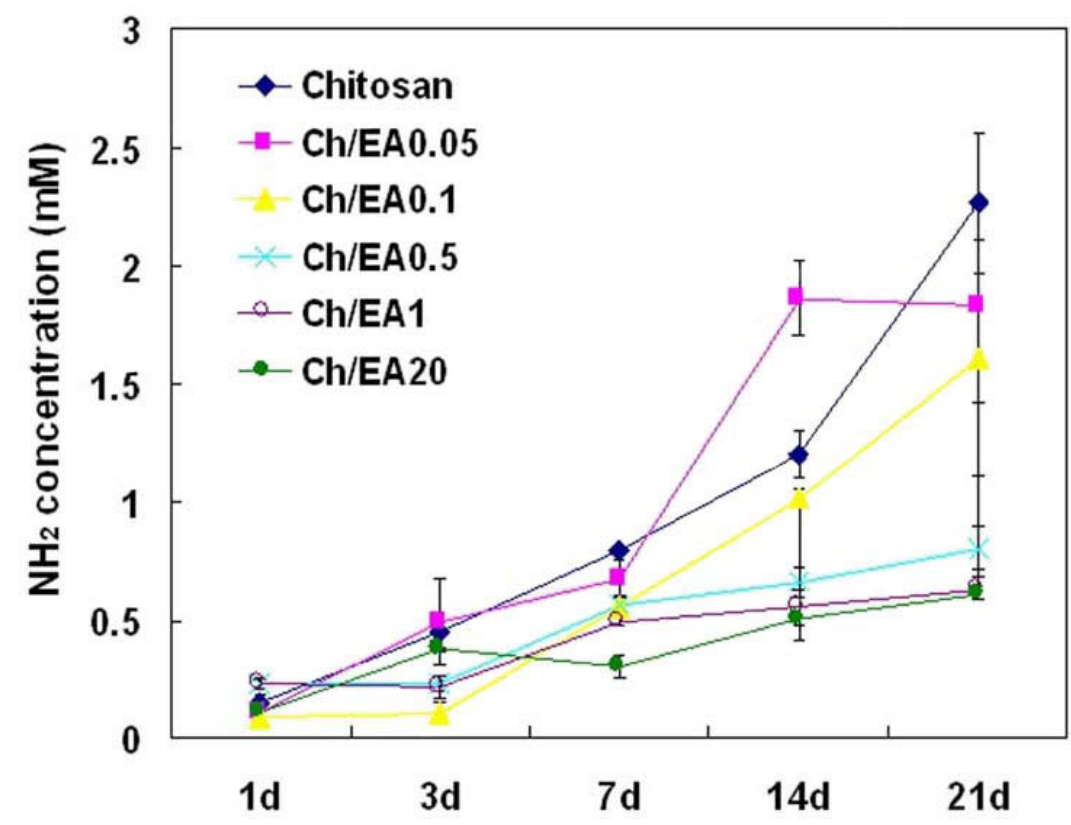

(b)

Figure 4.2 In vitro enzymatic degradation of the $\mathrm{Ch} / \mathrm{EA}$ composite films. (a) Same initial total weight and (b) Same initial chitosan weight. The amount of free amino groups corresponding to $\mathrm{N}$-glucosamine was determined as a function of time by a ninhydrin assay at $550 \mathrm{~nm}$. Each value represents the mean $\pm \mathrm{SD}(\mathrm{n}=3$ per each group). 


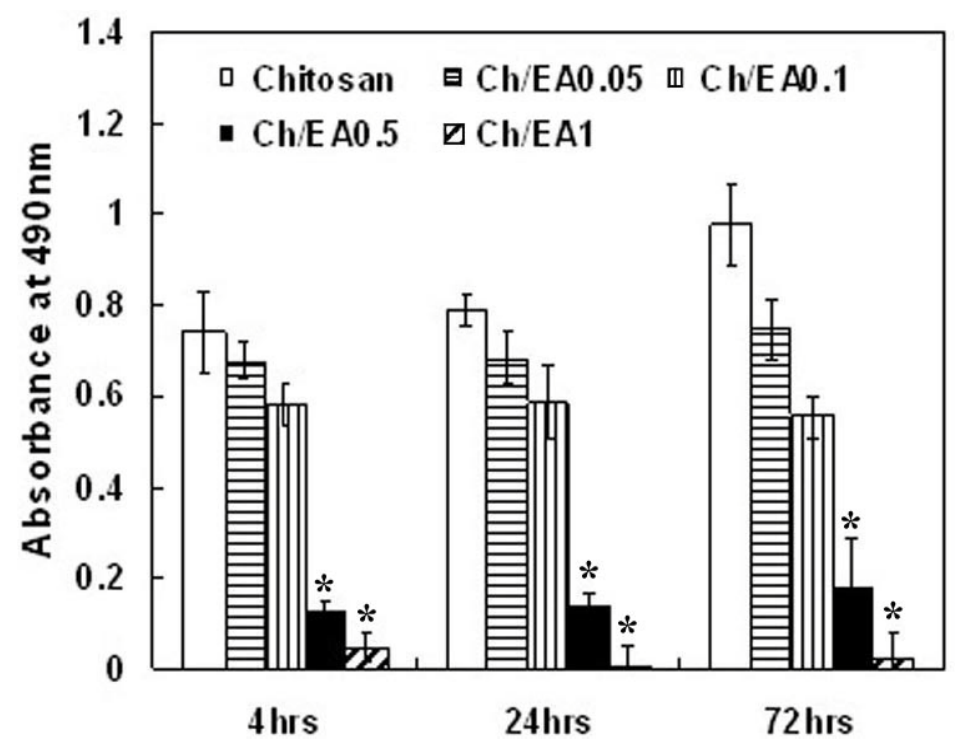

(a)

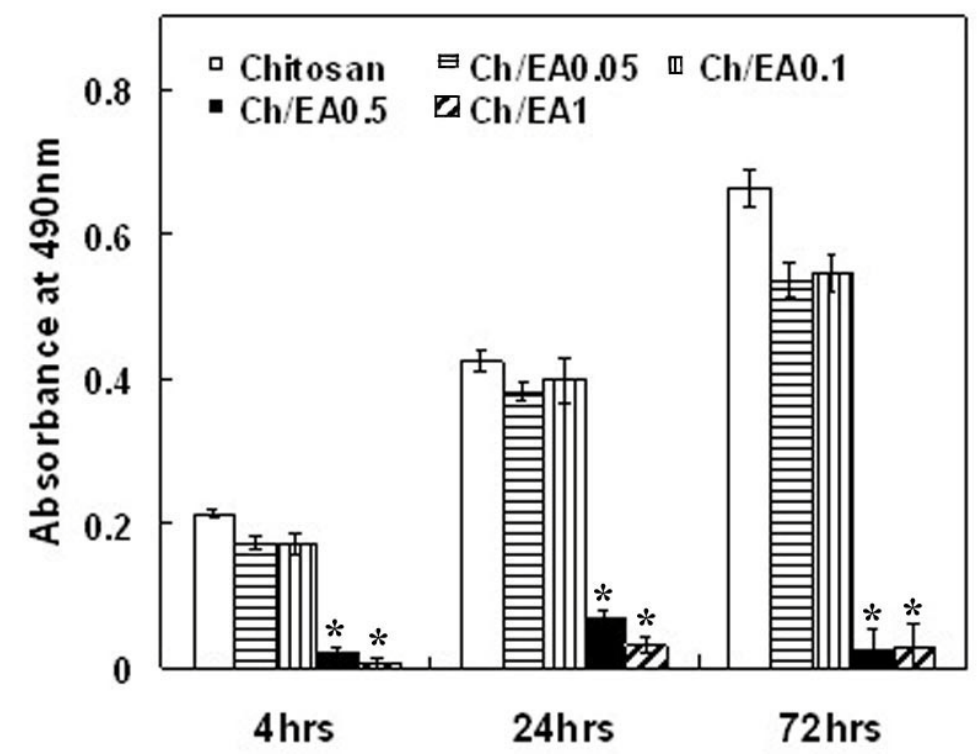

(b)

Figure 4.3 Viability of (a) human U87 glioblastoma and (b) rat C6 glioma via direct contact culture by MTS assay. The cells were cultured directly on each sample at a density of 30,000 cells per well without changing medium for 4, 24, and 72 hours. The absorbance is expressed as a measure of the cell viability on the chitosan coatings with varying concentration of the ellagic acid for 3 days incubation. The initial cell density was 30,000 cells per well. Each value represents the mean $\pm \mathrm{SD}(\mathrm{n}=4$ per each group). * denotes significantly different cell viability compared with the chitosan film, Ch/EA0.05, and $\mathrm{Ch} / \mathrm{EA} 0.1$ at 4, 24, and 72 hours of the incubation $(p<0.05)$. 
on the chitosan film and Ch/EA0.05 after 72 hours incubation compared with 4 hours incubation $(p<0.05)$. However, the viability of the U87 cells was slightly decreased when treated with the Ch/EA0.1 for 72 hours incubation compared with 4 hours (Figure 4.3a), but no significant change was observed $(p=0.17)$. As shown in Figure $4.3 \mathrm{~b}$, a significant increase in the viability of C6 glioma cells on the Ch/EA0.1 was observed after 72 hours compared to 4 and 24 hours incubation $(p<0.05)$. This result suggested that there was no inhibitory effect of the $\mathrm{Ch} / \mathrm{EA} 0.1$ on the survival and growth of cancer cells.

However, the treatment by the $\mathrm{Ch} / \mathrm{EA} 0.5$ and $\mathrm{Ch} / \mathrm{EA} 1$ induced significant decreases in viabilities of the both cancer cells within few hours compared with the other treatments $(p<0.05)$, indicating anti-cancer effects as shown in Figure $4.3 \mathrm{a}$ and $\mathrm{b}$. This result suggested that $\mathrm{Ch} / \mathrm{EA}$ composite films could inhibit the growth of cancer cells in an ellagic acid concentration dependent manner.

Figure 4.4 and 4.5 display the morphological changes of the U87 glioblastoma and C6 glioma cells on the chitosan film, Ch/EA0.1, and Ch/EA1 at 1 and 3 days incubation. After 1 day incubation, the U87 glioblastoma cells aggregated and were viable on the chitosan film and $\mathrm{Ch} / \mathrm{EA} 0.1$ as shown in Figure 4.4a and c. This observation indicated that cancer cells could not proliferate well due to initial inhibitory effect of films. However, the U87 glioblastoma cells did not aggregate and lost their viability on the $\mathrm{Ch} / \mathrm{EA} 1$ (Figure 4.4e).

After 3 days incubation, the U87 glioblastoma cells spread and proliferated on the chitosan film (Figure 4.4b) while they showed bigger aggregation in size on Ch/EA0.1 (Figure 4.4d). However, a few viable U87 glioblastoma cells were shown on the Ch/EA1 (Figure 4.4f). Similarly, the C6 glioma cells initially aggregated and then spread on the chitosan film and $\mathrm{Ch} / \mathrm{EA} 0.1$ after 3 days incubation as shown in Figure $4.5 \mathrm{a}-\mathrm{d}$, indicating the growth and proliferation of the glioma cells. However, the C6 glioma cells could not survive and grow on the Ch/EA1 as shown in Figure 4.5e and $\mathrm{f}$.

\subsubsection{Cell Viability - Indirect Contact}

The indirect contact culture was used to evaluate the effect of the ellagic acid released from the $\mathrm{Ch} / \mathrm{EA} 1$ on viability of U87 glioblastoma and C6 glioma cells for 4, 24, and 72 hours incubation. As shown in Figure 4.6, both cancer cell lines significantly proliferated in the absence of direct contact after 72 hours incubation compared to 4 hours $(p<0.05)$. The $\mathrm{Ch} / \mathrm{EA} 1 \mathrm{did}$ not affect growth and proliferation of cancer cells through the indirect cell culture for 3 days incubation periods. This result indicated that the growth of U87 glioblastoma and C6 glioma cells were significantly affected by close contact of the $\mathrm{Ch} / \mathrm{EA}$ composite film in an ellagic acid concentration dependent manner. Therefore, anti-tumor effect of the composite film might be localized at the targeted tissue area due to slow release of ellagic acid into surrounding area. However, there was significant difference between chitosan film and Ch/EA1 at 3 days incubation $(p<0.05)$. This result indicated the released amount of ellagic acid from Ch/EA 1 influenced slowly both cancer cells for 3 days incubation. 


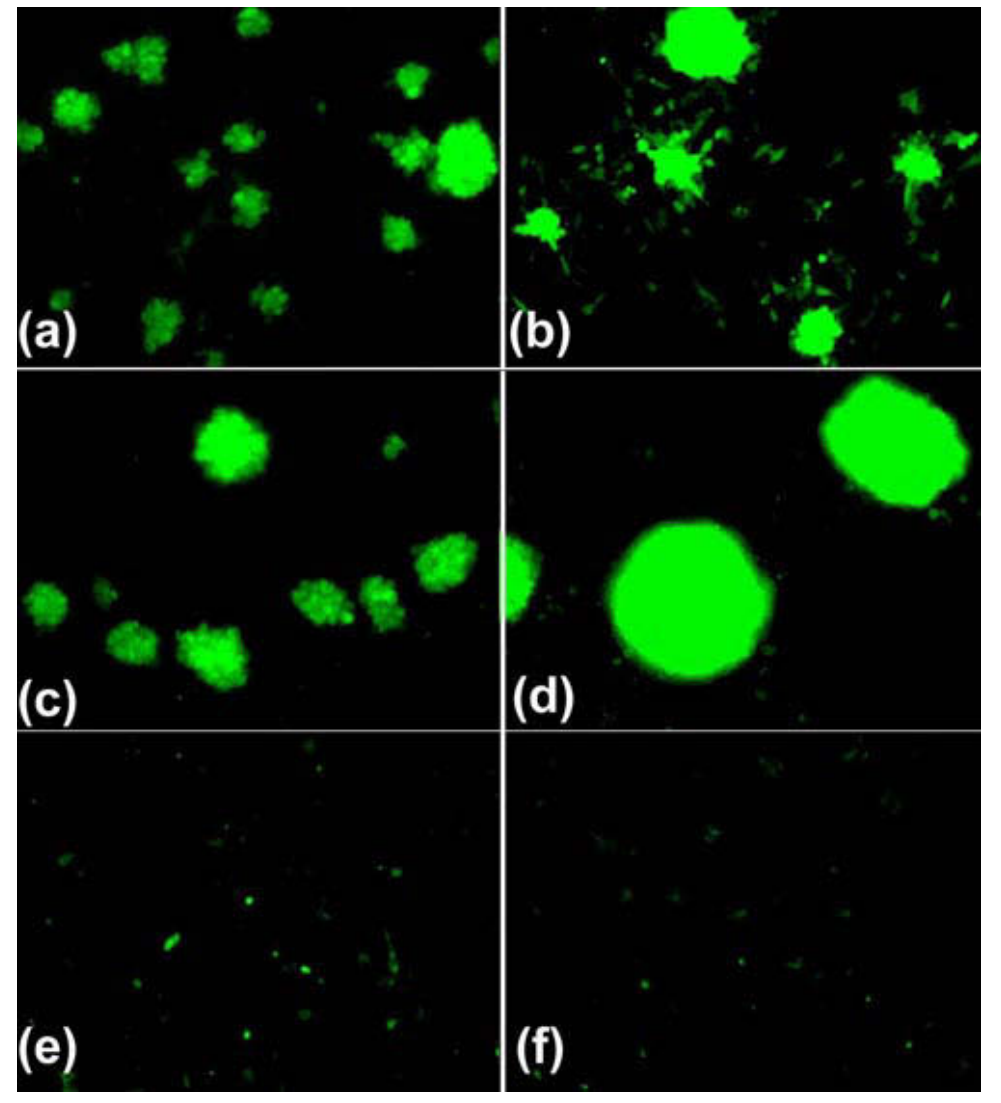

Figure 4.4 Fluorescent images of GFP tagged human U87 glioblastoma. (a) chitosan film for 1 day; (b) chitosan film for 3 days; (c) Ch/EA0.1 for 1day; (d) Ch/EA0.1 for 3 days; (e) $\mathrm{Ch} / \mathrm{EA} 1$ for 1day; (f) $\mathrm{Ch} / \mathrm{EA} 1$ for 3 days. The initial cell density was 30,000cells per well. $(\mathrm{MAG}=\times 100)$. These fluorescent images were generated by pseudo-color using MetaVue software after having been taken using a microscope (Nikon ECLIPSE TE2000U). 


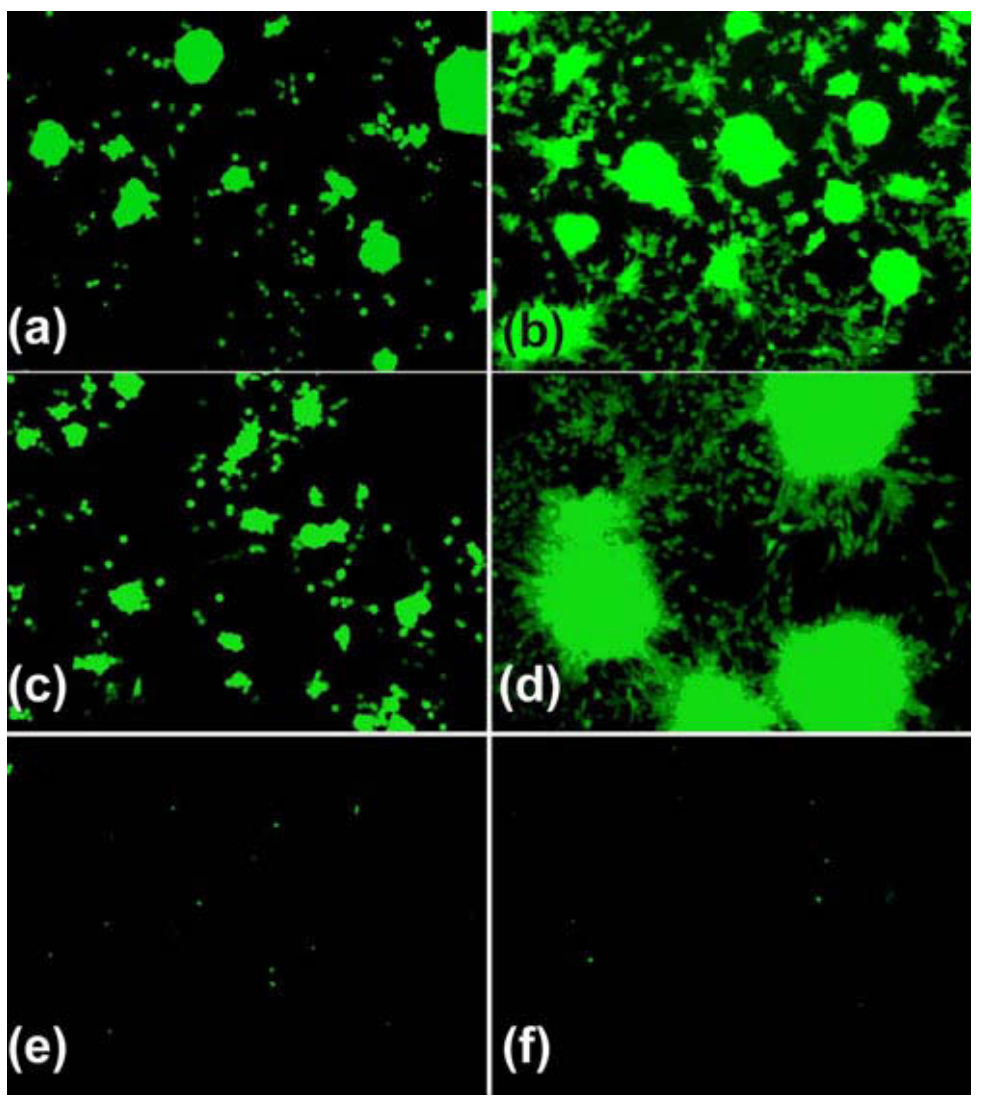

Figure 4.5 Fluorescent images of GFP tagged rat C6 glioma. (a) chitosan film for 1 day; (b) chitosan film for 3 days; (c) Ch/EA0.1 for 1day; (d) Ch/EA0.1 for 3 days; (e) Ch/EA1 for 1day; (f) Ch/EA1 for 3 days. The initial cell density was 30,000cells per well. (MAG $=\times 100$ ). These fluorescent images were generated by pseudo-color using MetaVue software after having been taken using a microscope (Nikon ECLIPSE TE-2000U). 


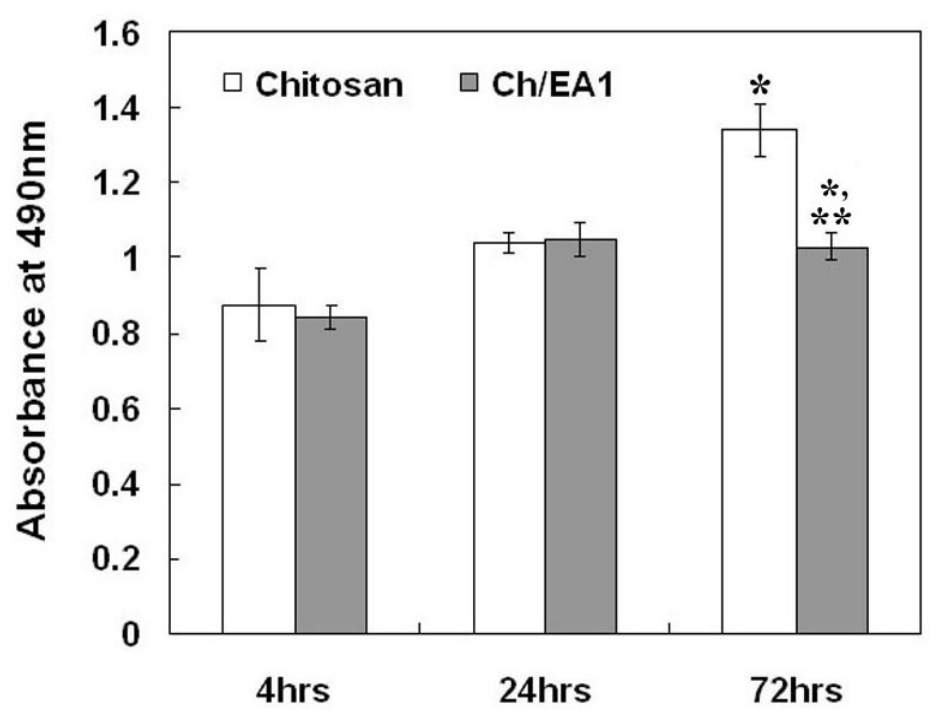

(a)

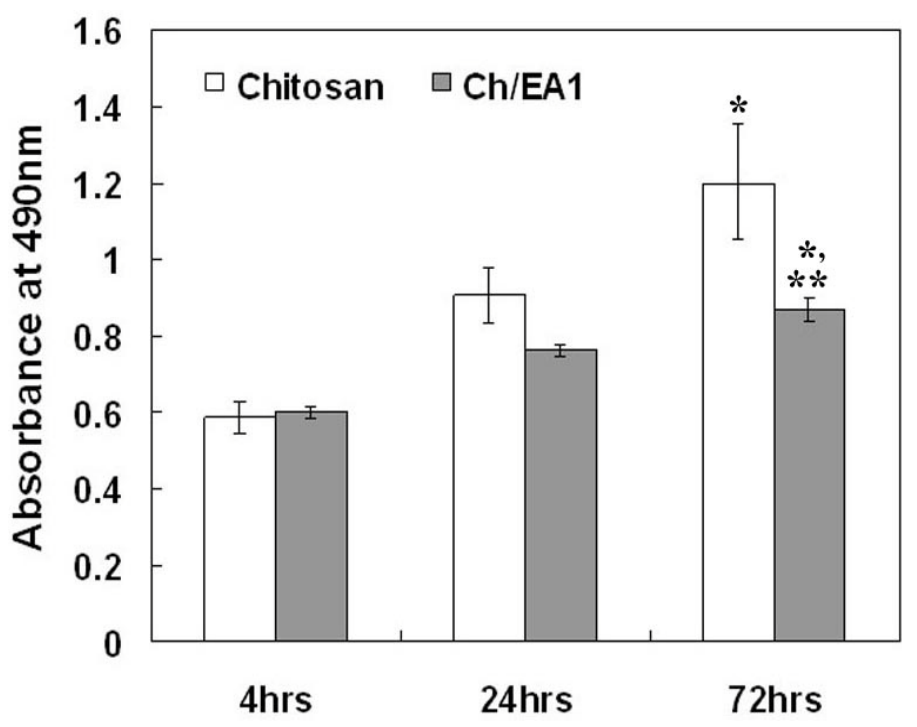

(b)

Figure 4.6 Viability of (a) human U87 glioblastomas and (b) rat C6 gliomas via indirect contact culture by MTS assay. The cells seeded onto the permeable PET membrane were affected by the released ellagic acid into the culture medium. The absorbance is an indicator of the viability of the cells on the Ch/EA1 for 4, 24, and 72 hours incubation. * denotes significantly different cell viability compared with 4 hours incubation $(p<0.05)$. $* *$ denotes significant difference compared with the chitosan film at 3 days incubation $(p<0.05)$. Each value represents the mean $\pm \mathrm{SD}(\mathrm{n}=3$ per each group). 


\subsubsection{Western Blot Analysis for p53}

The tumor suppressor protein p53 plays an important role in regulating survival and death of cancer cells. In response to some factors such as DNA damage, the level of p53 protein rises and induces cell cycle arrest or apoptosis. In order to investigate the mechanism by which the $\mathrm{Ch} / \mathrm{EA}$ composite films inhibit cancer cell growth, the level of p53 in rat C6 glioma cells was examined after treatments. As shown in Figure 4.7, the p53 protein became detectable in rat C6 glioma cells after a treatment with the Ch/EA1 while it did not appear to the untreated cells. This result demonstrated the Ch/EA composite films could induce the accumulation of the tumor suppressor protein $\mathrm{p} 53$, which likely preceded an induction of apoptosis.

\subsubsection{Caspase-3 Activation}

Caspase-3 is an active cell death protease and activated in the execution phase of cell apoptosis. In order to identify whether the $\mathrm{Ch} / \mathrm{EA}$ composite film induce apoptotic cell death, the effect of treatments (chitosan film or Ch/EA1) on caspase-3 activation was investigated in the U87 human glioblastoma and C6 rat glioma cells.

As shown in Figure 4.8, caspase-3 activation was significantly increased when both cancer cell lines were treated with the Ch/EA1 for 6 hours $(p<0.05)$, followed by the $\mathrm{Ch} / \mathrm{EA} 1$ for 2 hours $(p<0.05)$ compared with an untreated group. This result indicated that caspase-3 activation was dependent on the time of treatment. There were significant increases in caspase- 3 activation of both cancer cell lines by the treatment of the chitosan film compared with the untreated group $(p<0.05)$. This result indicated that the chitosan film has an ability to influence the growth of cancer cells. However, the effect of the chitosan film on the caspase-3 activation was significantly lower compared with that of the $\mathrm{Ch} / \mathrm{EA} 1$. This finding was consistent with the result of the cell viability measured by MTS assay shown in Figure 4.3, and suggested that Ch/EA composite films could induce apoptotic cell death within a few hours of interaction via the caspase- 3 activation.

\subsubsection{In vitro Angiogenesis Assay}

Tube formation is a multi-step process involving adhesion, migration, differentiation and growth of cells. This study was to examine the ability of the Ch/EA composite film to inhibit the growth of HUVEC using a widely used angiogenesis assay. Figure 4.9 shows the tube formations of the HUVEC in response to the different treatments. The HUVEC were allowed to grow and differentiate in the gel matrix for 8 hours after treatments. As shown in Figure 4.9a and b, when the HUVEC were cultured without treatment or treated with a chitosan film, the cells aligned themselves end to end, elongated and formed interconnected networks of cells because the matrix components influenced the behavior of cells. However, when the HUVEC were treated with the $\mathrm{Ch} / \mathrm{EA} 1$ for 1 day (Figure 4.9c), they did not change their morphology and tube formation was not observed. 


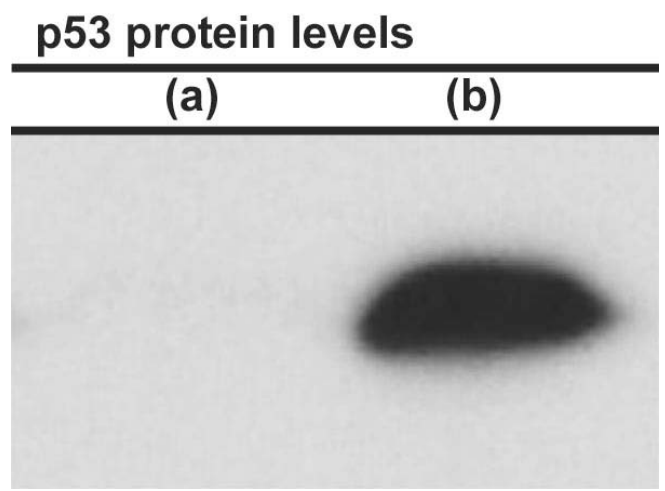

Figure 4.7 Western blot analysis of the p53 protein levels in the rat C6 glioma cells. (a) untreated and (b) treated with the Ch/EA1 for 1 day. 


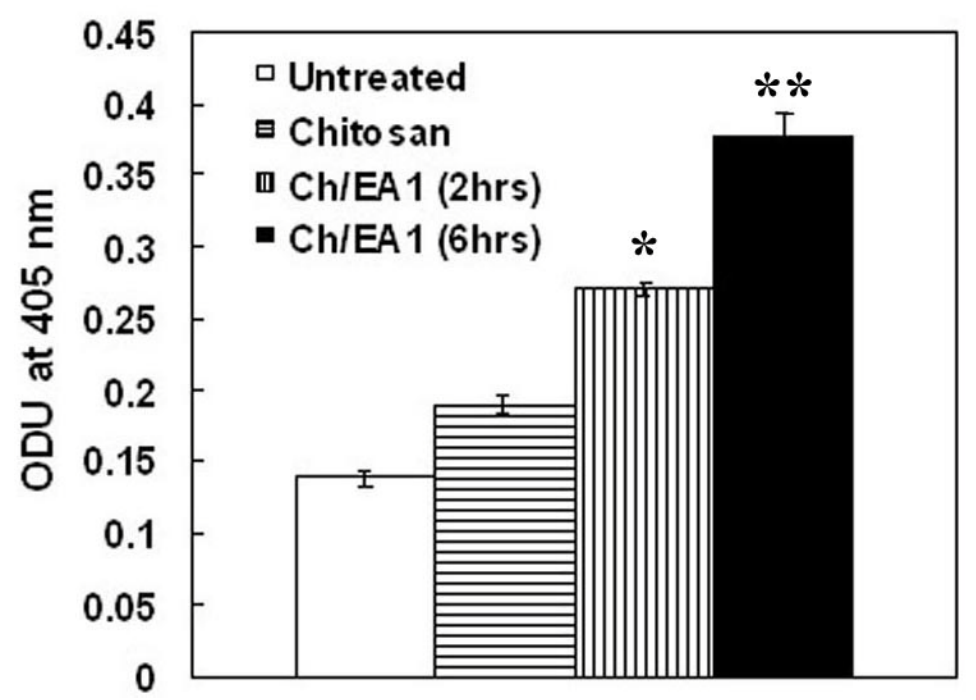

(a)

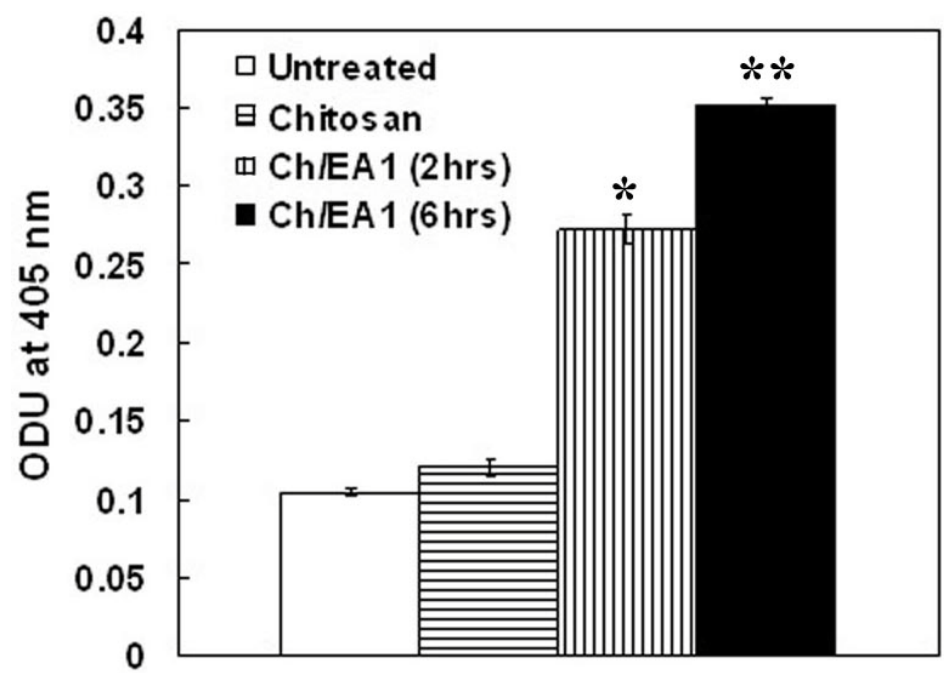

(b)

Figure 4.8 Caspase-3 activity after treatment. (a) human U87 glioblastoma and (b) rat C6 glioma cells (untreated; chitosan film for 6 hours; Ch/EA1 for 2 or 6 hours). ${ }^{*}$ denotes significantly increased caspase-3 activity compared with the untreated and the chitosan film $(p<0.05)$. ** denotes significantly increased caspase-3 activity compared with 2 hours incubation $(p<0.05)$. Each value represents the mean $\pm \mathrm{SD}(\mathrm{n}=3$ per group). 


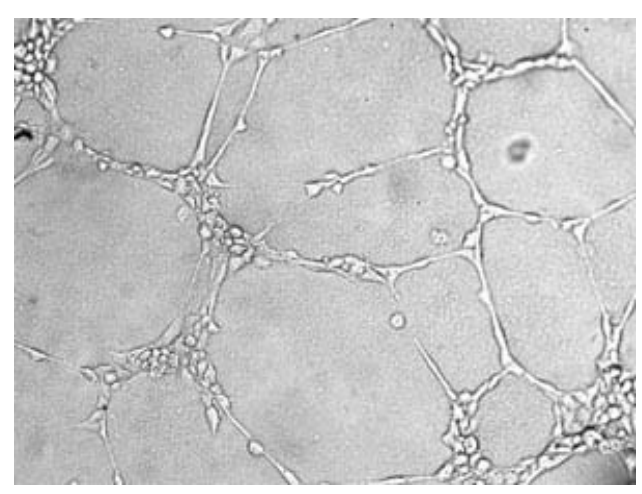

(a)

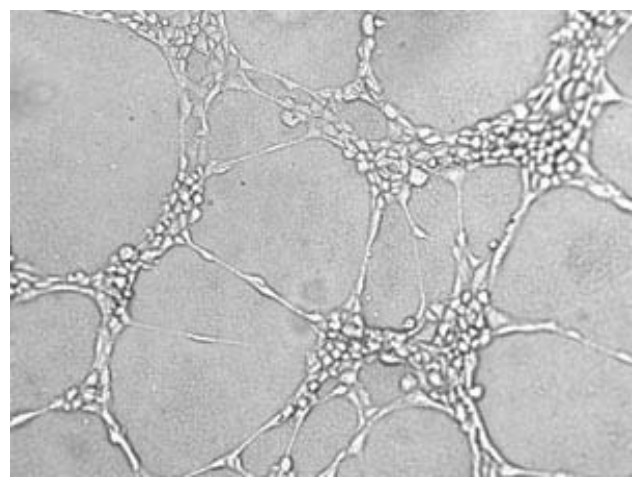

(b)

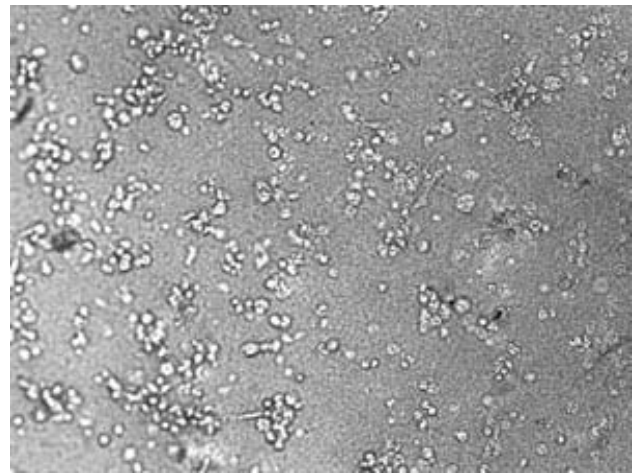

(c)

Figure 4.9 In vitro angiogenesis assay to investigate the morphological differentiation of HUVEC into capillary-like structures. Cells were (a) untreated; (b) treated with chitosan film for 1 day; (c) Ch/EA1 for 1 day. The HUVEC (10,000 cells per well) were cultured on a gel matrix for 8 hours after each treatment. The endothelial network formation was observed and photographed under a phase contrast inverted microscope (Nikon ECLIPSE TE-2000U). 
This was probably because the cells lost their viability after treatment with the $\mathrm{Ch} / \mathrm{EA} 1$ for 1 day. This result demonstrated that the chitosan/ellagic acid composite film (Ch/EA1) was effective at inhibiting morphological differentiation of the HUVEC, indicating the effect of the $\mathrm{Ch} / \mathrm{EA}$ composite film on suppressing angiogenesis.

\subsubsection{In vivo Anti-Tumor Activity}

In order to study the in vivo anti-tumor effect of Ch/EA composite films, the primary tumors were treated at an early stage. The treatment was initiated by implanting films (chitosan or Ch/EA20) subcutaneously on the fifth day after tumor inoculation. Tumor growth was evaluated by measuring tumor volume using a caliper, an ultrasound machine, and an optical imaging system every two days after treatment. Tumor growth was measured in real-time without sacrificing animals. All size measurements were determined using the largest diameter of the tumor tissue and its largest perpendicular diameter (Figure 4.10).

The result demonstrated that the $\mathrm{Ch} / \mathrm{EA} 20$ group significantly inhibited the growth of tumor tissue compared with the untreated group and chitosan control group over 3 weeks observation $(p<0.05)$. The different measurements showed different volume increases in the tumor tissues with time, but all the results indicated that Ch/EA20 group significantly delayed the growth rate of the tumor tissue compared with the other groups. Using a caliper measurement as shown in Figure 4.11, the difference of the tumor volume became statistically significant between the Ch/EA20 and untreated group from day 9 after tumor inoculation $(p<0.05)$.

The difference was statistically significant between the Ch/EA20 and the chitosan group from day 15 after tumor inoculation $(p<0.05)$. There was also a significant difference between the untreated and the chitosan group at day 11 and 13 after tumor inoculation $(p<0.05)$. However, there was no significant difference between the untreated and the chitosan group at the end of an experiment period.

Using an ultrasound measurement as shown in Figure 4.10b, a cross sectional area of a tumor tissue was imaged, and the two largest perpendicular diameters were measured and calculated according to formula [Li 2004]. As shown in Figure 4.12a, there was a significant difference between the Ch/EA20 group and untreated group from day 9 after tumor inoculation $(p<0.05)$. The difference was statistically significant between the Ch/EA20 group and the chitosan group from day 9 after tumor inoculation $(p<0.05)$. There was no significant difference between the untreated and the chitosan group during the experimental periods. Figure $4.12 \mathrm{~b}$ shows the ultrasound scan images indicating different tumor volumes among groups. This result demonstrated that the $\mathrm{Ch} / \mathrm{EA} 20$ film could significantly delay the growth rate of the glioma tumor compared with the other groups.

Using optical measurement as shown in Figure 4.10c, density differences of the tumor tissue was imaged using fluorescent emission. 


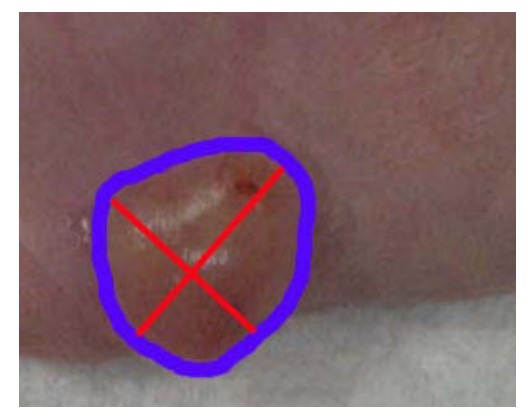

(a)

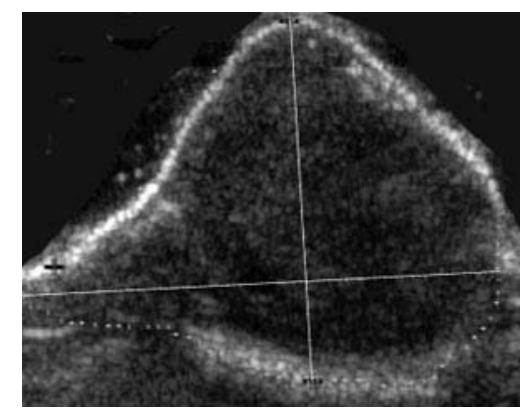

(b)

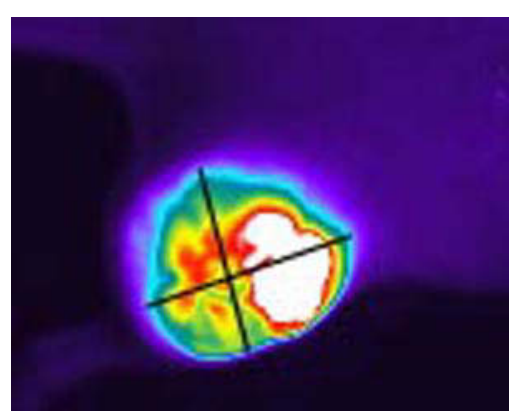

(c)

Figure 4.10 The measure of tumor volume using (a) caliper; (b) ultrasound machine; (c) fluorescence reflectance imaging (FRI) system. Each mouse was inoculated subcutaneously with GFP tagged rat C6 glioma cells at the density of $4 \times 10^{6}$ cells in the right flank. The cross-product of the two largest perpendicular diameters was calculated: tumor volume $\left(\mathrm{mm}^{3}\right)=($ major axis $) \times(\text { minor axis })^{2} \times 0.52$. The major axis is the longest diameter of the tumor and the minor axis is the maximal line drawn perpendicular to the major axis. 


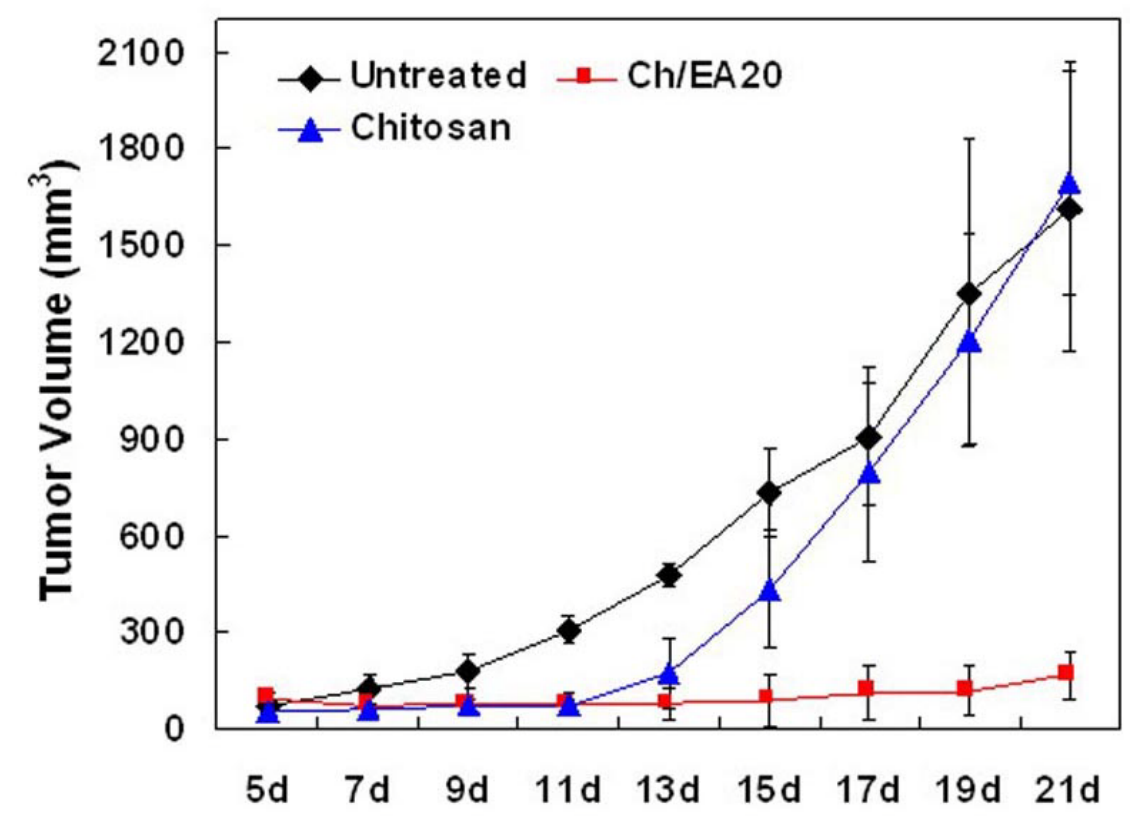

Figure 4.11 In vivo anti-tumor efficacy comparisons using a caliper. The treatments were initiated on day 5 after tumor inoculation as a function of time. Untreated tumor-bearing group (closed diamond, $\mathrm{n}=5$ ), chitosan control group (closed triangle, $\mathrm{n}=5$ ), and $\mathrm{Ch} / \mathrm{EA} 20$ experimental group (closed square, $\mathrm{n}=5$ ). 
Figure 4.12 In vivo anti-tumor efficacy comparisons using an ultrasound machine. The effect of treatments on tumor growth was evaluated by (a) volume measurements; (b) ultrasound images. The treatments were initiated on day 5 after tumor inoculation as a function of time. Untreated tumor-bearing group (closed diamond, $\mathrm{n}=5$ ), chitosan control group (closed triangle, $\mathrm{n}=5$ ), and $\mathrm{Ch} / \mathrm{EA} 20$ experimental group (closed square, $\mathrm{n}$ $=5$ ) were compared. Ultrasound scan images show the nude mice with GFP tagged rat C6 glioma on each right flank at the 5, 13, and 21 days after tumor inoculation. 


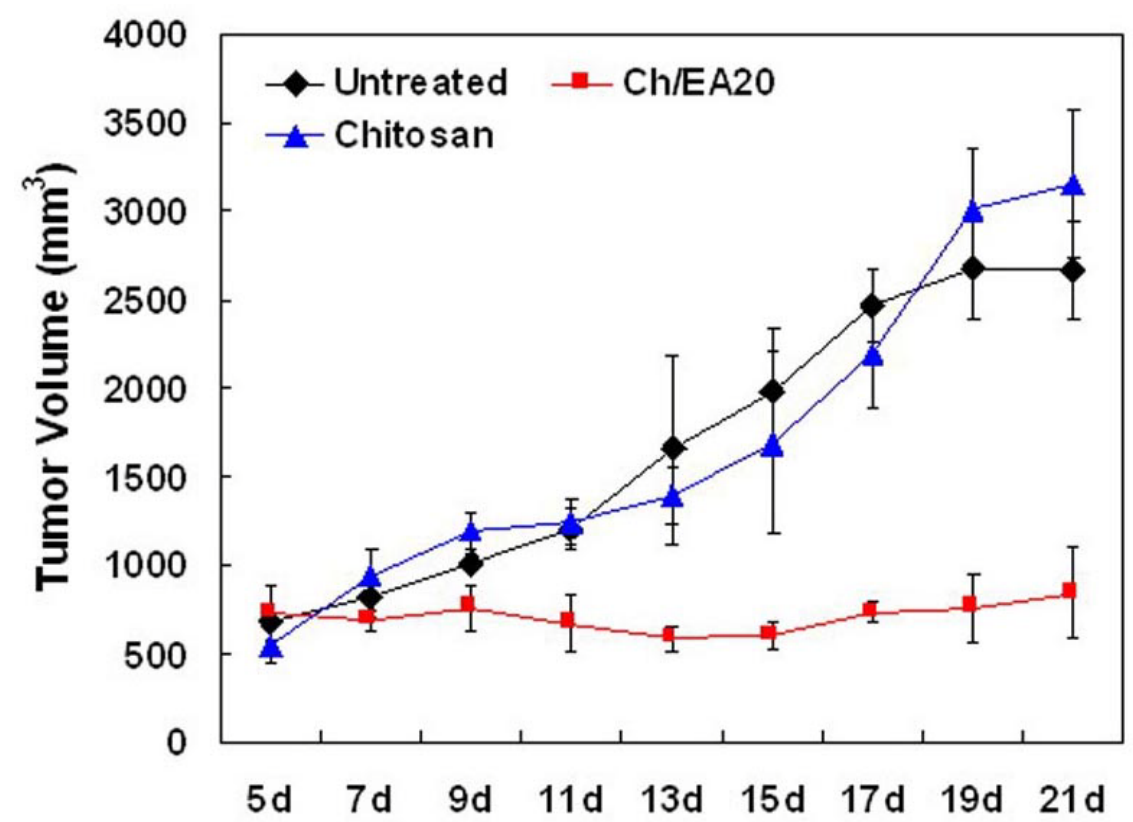

(a) 


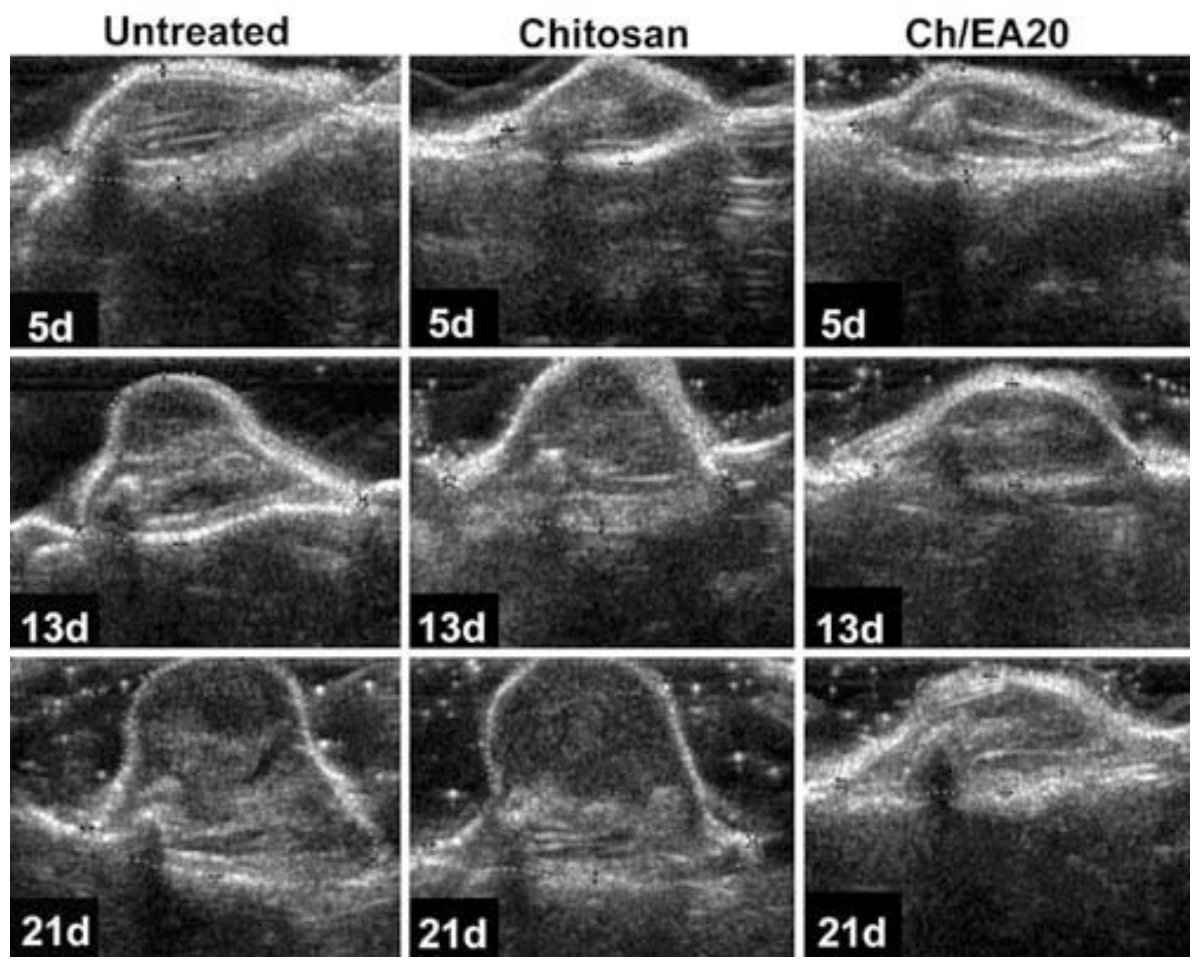

(b)

Figure 4.12 (continued). 
Higher intensity of fluorescent emission represented higher tumor population. As shown in Figure 4.13a, there was a significant difference between the Ch/EA20 and the untreated group from day 9 after tumor inoculation $(p<0.05)$. The difference was statistically significant between the $\mathrm{Ch} / \mathrm{EA} 20$ and the chitosan group for day $15,17,19$ and 21 after tumor inoculation $(p<0.05)$. There was also a significant difference between the untreated and the chitosan group at day 9 and 11 after tumor inoculation $(p<0.05)$. However, no significant difference in tumor growth was observed between the untreated group and the chitosan group for day 15, 17, 19 and 21 after tumor inoculation. The optical imaging clearly showed a smaller tumor volume in the Ch/EA20 group compared with those in the untreated and a chitosan film group (Figure 4.13b), indicating an effective suppression of the tumor growth in the $\mathrm{Ch} / \mathrm{EA} 20$ group.

As shown in Figure 4.14, there was a significant difference $(p<0.05)$ in weight loss between the Ch/EA20 and the other groups from day 5 to day 7 of the experiment. The mice treated with the Ch/EA20 films displayed higher weight loss at the first 1-2 days after treatments compared with the other groups, but they had a weight curve similar to those of the other groups. Weight loss was less than $10 \%$ for all the groups, indicating no severe toxic side effects on animals during the period of experiments.

As shown in Figure 4.15 and 4.16, the efficacy of the local delivery of ellagic acid was confirmed by the histological analysis of tumors. For the tumor bearing group (untreated) and the chitosan group, the tumor cells were aggressive, invasive, and highly populated. Mostly, the center of tumors experienced necrosis (cell fragments and nuclear debris). For the $\mathrm{Ch} / \mathrm{EA} 20$ group, it was observed that there were a decrease in tumor cell population and a formation of fibrous tissue surrounding the material. The mixture of a few inflammatory cells and necrotic cells was also found, indicating a sort of foreignbody reaction. In tumor cell zone close to the material, tumor cells were not aggressive but less populated. The result demonstrated that the $\mathrm{Ch} / \mathrm{EA} 20$ did not induce severe inflammatory response but reduced the population of invasive tumor cells around the $\mathrm{Ch} / \mathrm{EA} 20$.

\subsection{Discussion}

To optimize therapeutic efficacy, numerous drug delivery strategies have been explored in terms of efficacy and safety. Especially, for brain cancer, the blood-brainbarrier (BBB) limits the access of therapeutic agents to brain tumor. In this regard, a local therapy combined with surgery provides a direct delivery of drugs into the brain, bypassing the BBB, thus improving efficacy. In the present study, $\mathrm{Ch} / \mathrm{EA}$ composite films were studied to treat glioma tumors via a local delivery of ellagic acid.

One of the limitations of conventional drug delivery is an initial burst effect. Drug release of biodegradable systems is achieved by diffusion via pores in the polymer matrix and polymer degradation [Dash 1998, Gunatillake 2003, Tomihata 1997]. In this study, the in vitro release profile of the ellagic acid from the $\mathrm{Ch} / \mathrm{EA}$ composite films clearly revealed a sustained slow release of ellagic acid both in the presence and absence 
Figure 4.13 In vivo anti-tumor efficacy comparisons using an optical imaging system. The effect of treatments on tumor growth was evaluated by (a) volume measurements; (b) fluorescence images. The treatments were initiated on day 5 after tumor inoculation as a function of time. Untreated tumor-bearing group (closed diamond, $\mathrm{n}=5$ ), chitosan control group (closed triangle, $\mathrm{n}=5$ ), and $\mathrm{Ch} / \mathrm{EA} 20$ experimental group (closed square, $\mathrm{n}$ $=5$ ). Fluorescence images show the nude mice with GFP tagged rat C6 glioma on each right flank at the 5, 13, and 21 days after tumor inoculation. 


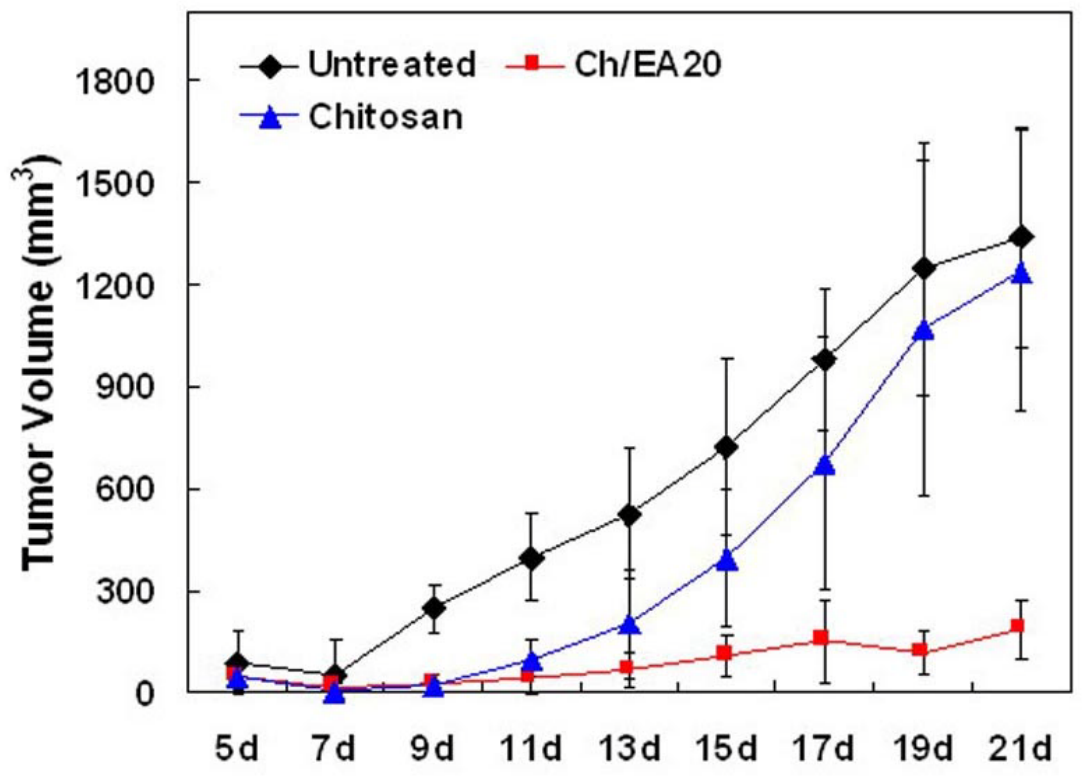

(a) 

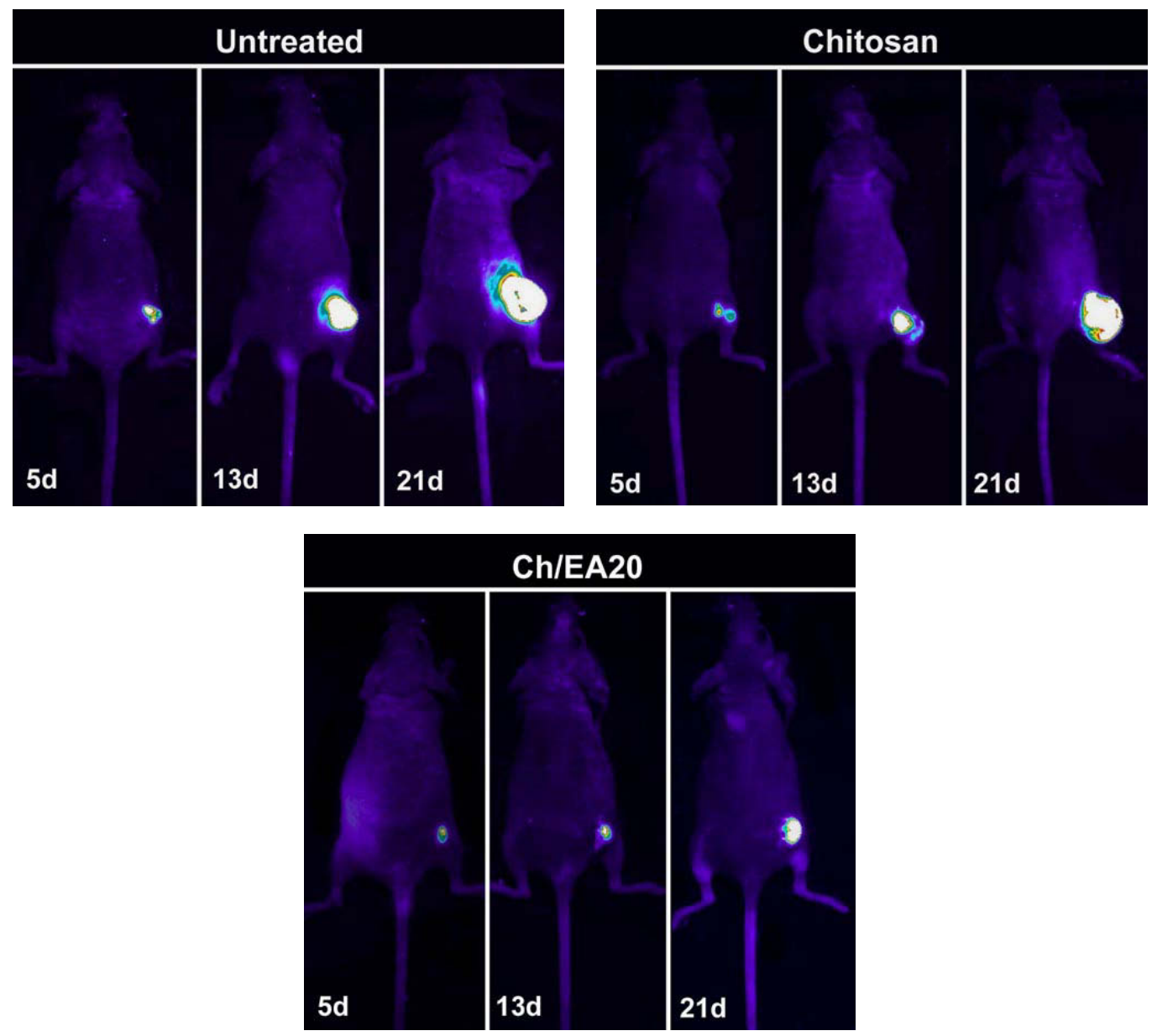

(b)

Figure 4.13 (continued). 


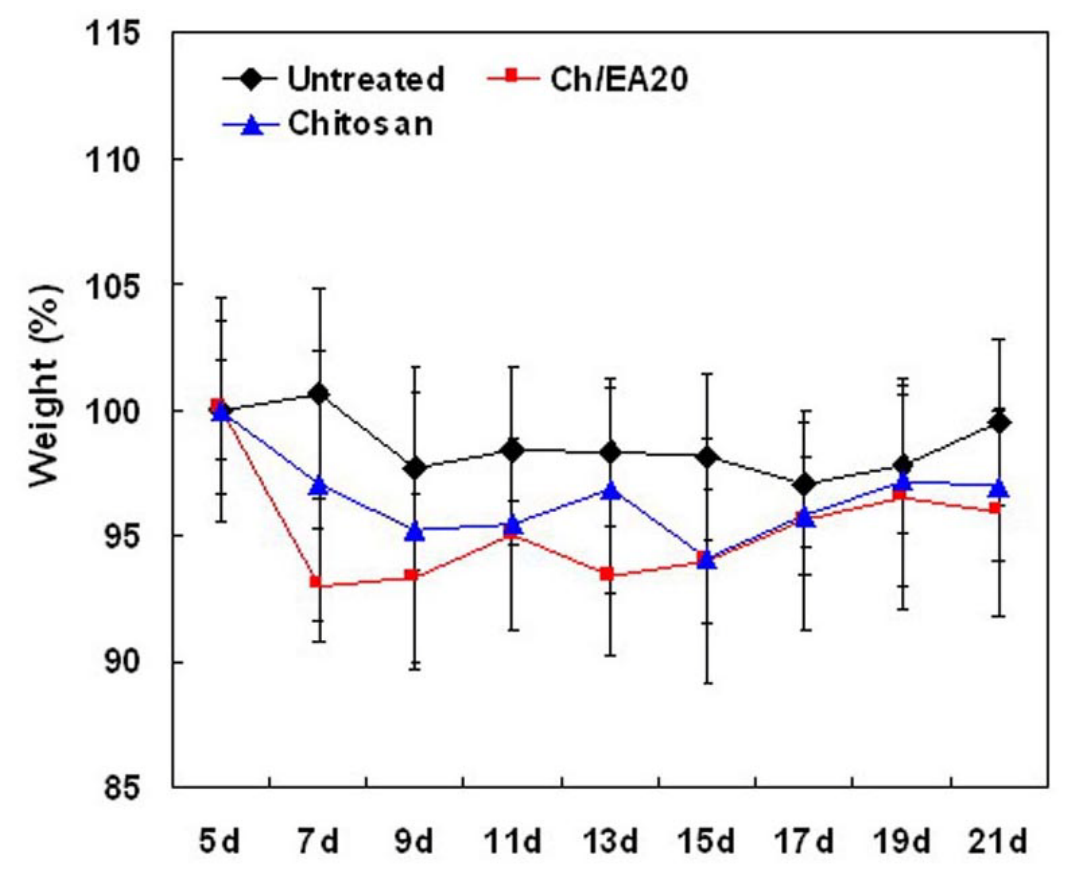

Figure 4.14 The percentage of weight loss of animals over time periods of treatments after tumor inoculation. Untreated tumor-bearing group (closed diamond, $n=5$ ); chitosan carrier control group (closed triangle, $\mathrm{n}=5$ ), and $\mathrm{Ch} / \mathrm{EA} 20$ experimental group (closed square, $n=5)$. 


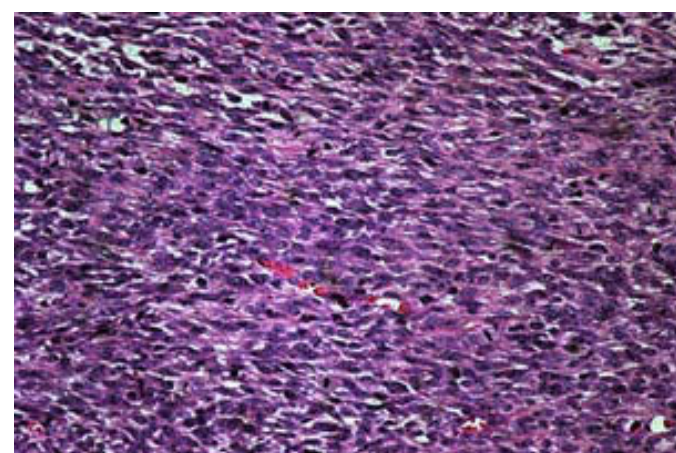

(a)

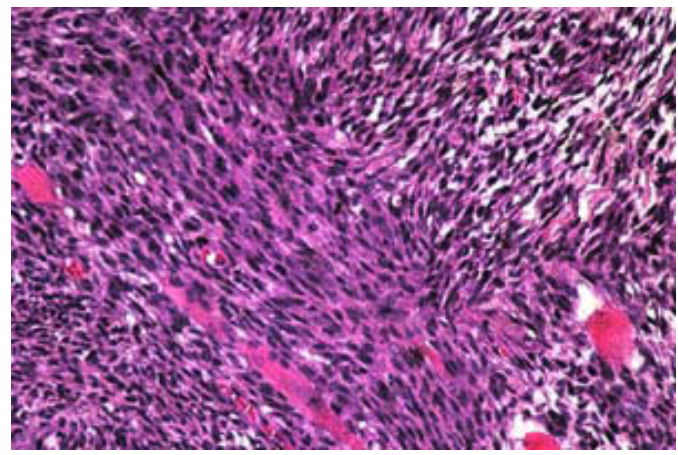

(c)

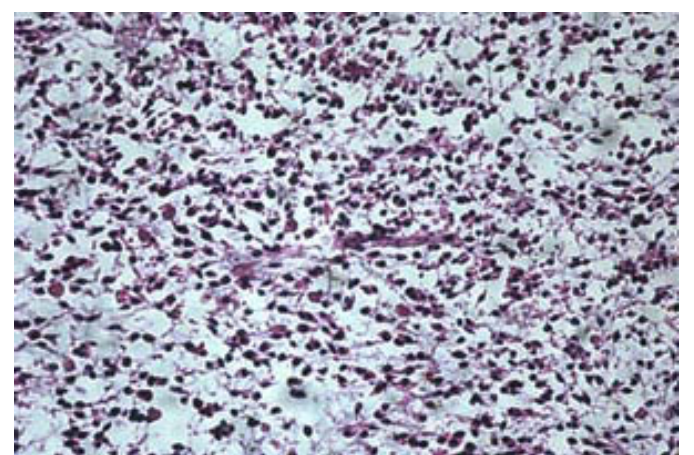

(b)

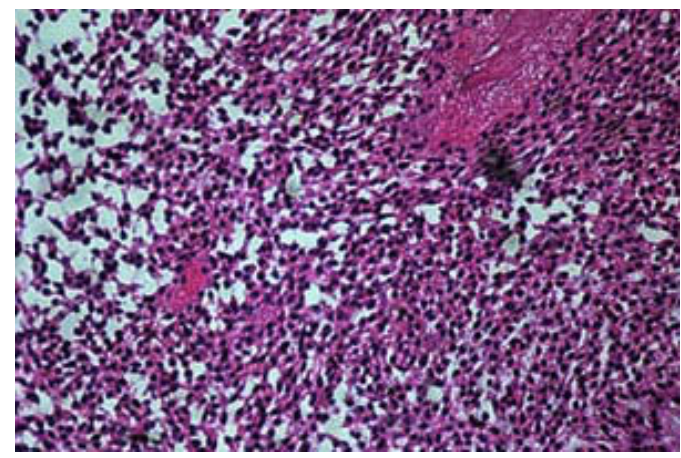

(d)

Figure 4.15 Histological analysis of rat C6 gliomas stained with hematoxylin and eosin. The tumor tissue cross sections represents the untreated tumor-bearing group with (a) viable and active tumor cells; (b) necrotic area; and the chitosan control group with (c) active tumor cells; (d) necrotic area. The samples were retrieved along with adjacent tissue after completion of the experiment (on day 21 after tumor inoculation). (MAG $=$ $\times 200)$. 


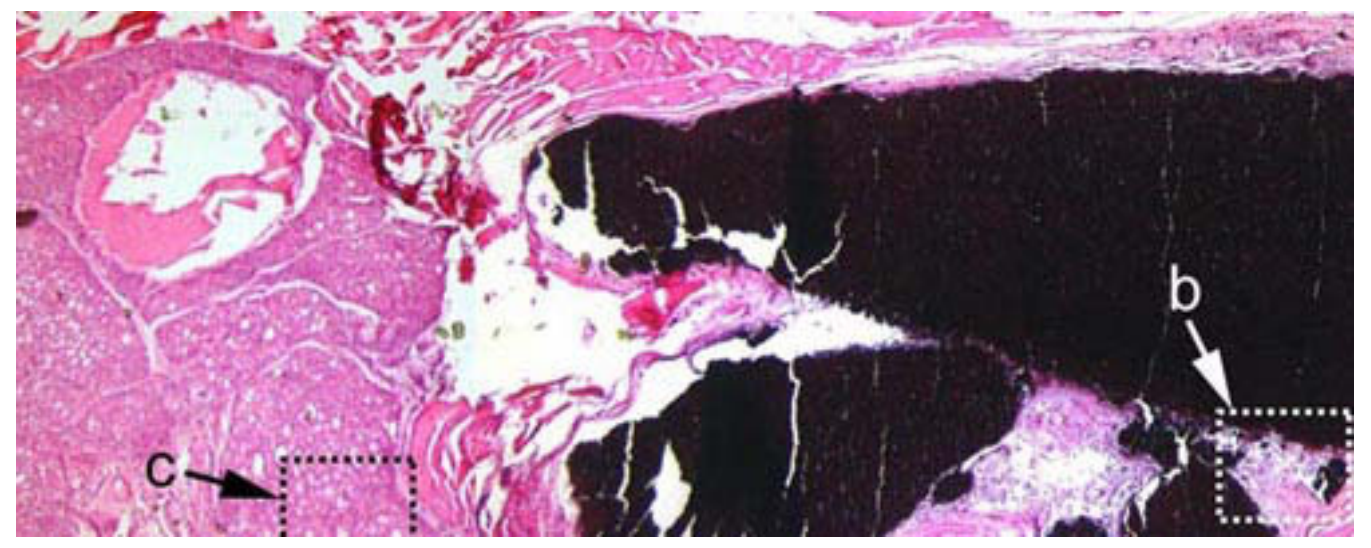

(a)

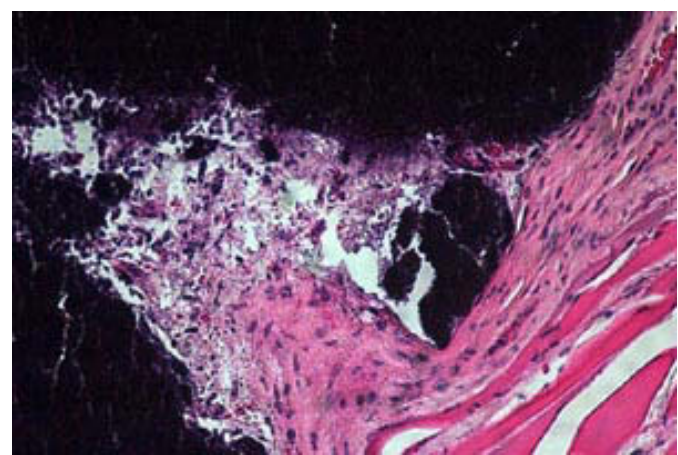

(b)

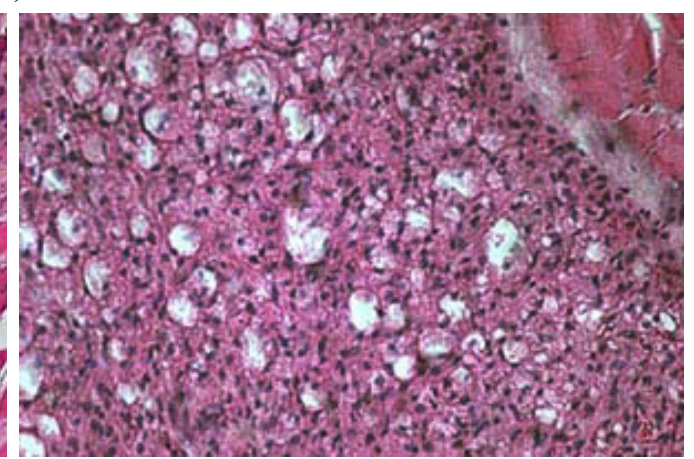

(c)

Figure 4.16. Histological analysis of rat C6 gliomas stained with hematoxylin and eosin. The tumor tissue cross sections of $\mathrm{Ch} / \mathrm{EA} 20$ experimental group represent (a) the material zone including the formation of the fibrous tissue and dead cells and the tumor cell zone $(\times 40)$; (b) the magnified material zone with a thin layer of fibrous tissue and dead cells $(\times 200)$; (c) the magnified tumor cell zone close to the material $(\times 200)$. The samples were retrieved along with adjacent tissue after completion of the experiment (on day 21 after tumor inoculation). 
of lysozyme. The presence of lysozyme could significantly accelerate the release of ellagic acid compared with the absence of lysozymes. The in vitro degradation study demonstrated that the enzymatic degradation rate of the $\mathrm{Ch} / \mathrm{EA}$ composite films was ellagic acid-concentration dependent. The composite films with higher concentration of ellagic acid (Ch/EA0.5, Ch/EA1, and $\mathrm{Ch} / \mathrm{EA} 20)$ exhibited slower degradation rate compared with lower concentration of ellagic acid (Ch, $\mathrm{Ch} / \mathrm{EA} 0.05$, and $\mathrm{Ch} / \mathrm{EA} 0.1)$. As discussed in chapter 3, ellagic acid was conjugated with chitosan polymer via covalent bonds or ionic forces and embedded into chitosan polymer network [Kim 2009]. Therefore, the degradation rate of $\mathrm{Ch} / \mathrm{EA}$ composite films corresponds with interactions between chitosan and ellagic acid.

In the previous study of degradation behavior, two $\mathrm{pH}$ values of 6 and 7.4 were examined. The result showed that the higher degradation rate occurred around $\mathrm{pH} 6$ in lysozyme-containing phosphate buffer solution compared with $\mathrm{pH}$ 7.4. In order to observe the differences among experimental groups in a timely manner, in this chapter, the phosphate buffer solution was prepared at $\mathrm{pH}$ 6. Chitosan is usually degraded via chitinase or lysozyme digestion. The fraction of $\mathrm{N}$-acetylglucosamine (NAG) units in chitosan contributes to its enzymatic hydrolysis [Freier 2005]. The ellagic acid on the surface of composite film inhibited the binding of lysozyme to the NAG units on the chitosan chains and slowed the reactions lysozymes catalyze, thereby decreasing the enzymatic hydrolysis rate in chitosan films. It is well known that the degradation of chitosan films is dependent on the degree of deacetylation (DDA) and molecular weight [Muzzarelli 1997, Pangburn 1982, Ren 2005]. Chitosan films that are deacetylated more than $73 \%$ showed a slow biodegradation rate [Khan 2002, Mi 2002]. Since a chitosan having a degree of deacetylation (more than $85 \%$ ) have been used, this characteristic of chitosan contributed to the slow release of ellagic acid. In addition to the low solubility of the chitosan carrier, ellagic acid is only slightly soluble in medium at $\mathrm{pH}$ 6.0. All the factors contribute to the sustained slow release rate of ellagic acid from films. In this regard, the release of ellagic acid from the composite films depends on characteristic of chitosan, fabrication methods, ellagic acid loadings, solubility, interactions between chitosan and ellagic acid, and medium (release environment) [Dash 1998].

This study investigated the viability of the human U87 glioblastomas and rat C6 glioma cells and the corresponding cell morphological changes on the composite films. In the study of direct cell culture, the $\mathrm{Ch} / \mathrm{EA}$ composite films showed anti-proliferative effect against cancer cells in an ellagic acid concentration-dependent manner. However, the cells in absence of direct contact with the composite films significantly proliferated. This is probably due to the slow release of the ellagic acid into the cell culture media as shown in the release study. These results clearly suggest that the $\mathrm{Ch} / \mathrm{EA}$ composite films have a localized anti-proliferative effect on cancer cells. This localized effect was also verified in the cell morphological changes at the interface of the composite films in chapter 3 [Kim 2009].

Ellagic acid can induce apoptotic process via the intrinsic mitochondrial pathway [Khan 2007, Mertens-Talcott 2005, Narayanan 1999]. The p53, a tumor suppressor protein is an important key protein involved in this pathway. The p53 protein arrests cell 
cycle and acts as pro-apoptotic signal. The p53 mediated activation of the pro-apoptotic proteins such as PUMA, NOXA, and BID induces the activation of caspases, leading to apoptosis [Bai 2006, Khan 2007]. In this study, p53 protein in the rat C6 gliomas and caspase-3 activation in the U87 human glioblastomas and the rat C6 gliomas were investigated. The results showed that the $\mathrm{Ch} / \mathrm{EA}$ composite films induced the accumulation of the tumor suppressor protein p53 and increase caspase- 3 activation. The findings of this study suggest the $\mathrm{Ch} / \mathrm{EA}$ composite film is able to suppress cancer growth by inducing apoptosis. Therefore, the induction of apoptosis by the composite films can minimize inflammatory response causing the damage in the surrounding tissue.

The angiogenesis is an essential step in tumor growth. The formation of new blood vessels associated with tumor growth via angiogenesis is a complex process. Endothelial cells are able to aggregate to form capillary-like structures. Besides the apoptosis mechanism, this study examined the ability of $\mathrm{Ch} / \mathrm{EA}$ composite films to inhibit the growth of HUVEC. In order to observe tube formation of endothelial cell, special gel membranes were used. These membranes are composed of laminin, collagen type IV, heparin sulfate, proteoglycans, entactin, and nidogen, and can accelerate the morphological differentiation of endothelial cells [Kubota 1988]. The endothelial cells on the basement membranes rapidly differentiate and start to form tubes within a few hours and complete by 1 day. This study demonstrated that a reconstituted gel membrane promoted morphological differentiation of the HUVEC into capillary-like structures when the cells were untreated or treated with the chitosan film. However, the HUVEC treated with $\mathrm{Ch} / \mathrm{EA} 1$ could not grow, differentiate, and form tubes. This study indicated that even though the basement membrane provided the key ingredients in the induction of angiogenesis, the $\mathrm{Ch} / \mathrm{EA} 1$ reduced the cell viability and suppressed angiogenesis.

Following the in vitro study, the in vivo anti-tumor activity and toxicity of the composite films were investigated using a mouse flank tumor model. To maximize the effect of this local delivery, the glioma tumors were treated at an early stage with films containing high concentrations of ellagic acid (Ch/EA20). Some studies [Au 2001, Bello 2002, Giussani 2003] demonstrated that the most significant effect on animal survival and suppression of tumor growth was dependent on the time at which treatment was initiated. A preliminary animal study, in which the treatment group (Ch/EA10) was implanted on the eleventh day after tumor inoculation, demonstrated there was no significant difference between a control group and a treatment group. This result suggests the significant effect corresponds with initial treatment time and concentration of ellagic acid. Therefore, in this study, treatment was initiated on the fifth day after tumor inoculation. At this point, the tumors were small and visually detectable.

This animal study demonstrated that the $\mathrm{Ch} / \mathrm{EA} 20$ group significantly inhibited tumor growth compared with the untreated tumor-bearing group and chitosan carrier control group. However, severe weight loss, an indicator of toxic side effect, was not observed in all the groups. In addition, the histological analysis also demonstrated the $\mathrm{Ch} / \mathrm{EA} 20$ did not induce any severe toxic effect on its surrounding area but a formation of fibrous tissue and dead cells. The tumor cell zone close to the $\mathrm{Ch} / \mathrm{EA} 20$ showed the population of the tumor cells which have lower density and tiny round shapes compared 
with the untreated group and chitosan group. This indicates the tumor cells were affected by the release of ellagic acid. The high efficacy and low toxicity of the treatment are attributed to the slow and localized release of ellagic acid from the composite film. These results are very encouraging and will allow us to further investigate the naturally-based delivery systems to locally administer naturally-based therapeutics to treat tumors, thereby improving both the safety and efficacy of cancer treatment.

\subsection{Conclusion}

This study showed the effect of a local delivery of ellagic acid against brain cancer in vitro and in vivo. The $\mathrm{Ch} / \mathrm{EA}$ composite films showed a sustained slow release of ellagic acid without an initial burst effect. They were enzymatically degradable, indicated by at least a 5 times higher concentration of free amino groups at 3 weeks incubation compared with 1 day. The presence of lysozyme and concentration of ellagic acid influenced the release profile of ellagic acid from the films. These composite films were able to induce apoptotic cell death in an ellagic acid concentration dependent manner and inhibit the morphological differentiation of the HUVEC in vitro. The $\mathrm{Ch} / \mathrm{EA}$ composite film also significantly suppressed glioma tumor growth in vivo. Tumor volume growth observed at 3 weeks was lower in the $\mathrm{Ch} / \mathrm{EA} 20$ group compared to control groups as measured with a caliper, an ultrasound machine, and an optical imaging system by 9 times, 3 times, and 7 times, respectively. This study demonstrated that the $\mathrm{Ch} / \mathrm{EA}$ composite film can treat glioma cancer using anti-cancer properties of ellagic acid in a local site. 


\section{CHAPTER 5. DEVELOPMENT AND EVALUATION OF A CHITOSAN BASED INJECTABLE GEL FOR BRAIN CANCER TREATMENT}

\subsection{Abstract}

A chitosan based injectable gel has been developed for local delivery of ellagic acid. Chitosan delivery of ellagic acid inhibits growth of brain cancer cells. The chitosan/ $\beta$-glycerophosphate solutions $(\mathrm{Ch} / \beta-\mathrm{GP})$ were prepared with different concentrations of $\beta$-GP and the ellagic acid was loaded to evaluate the anti-tumor effect of the gel on brain cancer cells (human U87 glioblastomas and rat C6 glioma cells). The properties of the $\mathrm{Ch} / \beta$-GP gels were investigated regarding chemical structure, surface morphology, and viscoelasticity. In vitro ellagic acid release studies were performed by monitoring UV absorbance and enzymatic degradation rate was determined by increase in free amino groups of chitosan. Cell number and activity were monitored by the MTS assay. The liquid $\mathrm{Ch} / \beta$-GP solution underwent thermal gelation at body temperature. The gelation temperature was dependent on the $\mathrm{pH}$ of the $\mathrm{Ch} / \beta$-GP solution rather than concentration of $\beta$-GP. The $\mathrm{Ch} / \beta$-GP gels were enzymatically degradable, showing the released free amino groups were at least 12 times higher at 3 weeks compared with 1 day. Ellagic acid release increased in the presence of lysozyme, indicated by at least a 2.5 times higher concentration of ellagic acid than that in the absence of lysozyme. Dialysis

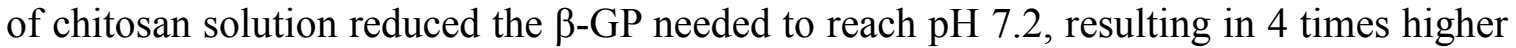
cell viability than undialyzed chitosan gel at 3 days culture. Viability of U87 cells and C6 cells cultured on chitosan gels containing $1 \%(\mathrm{w} / \mathrm{v})$ of ellagic acid were lower than the same cells on chitosan gels at 3 days incubation by 3.8 times and 6.5 times, respectively.

\subsection{Introduction}

Conventional chemotherapy non-specifically distributes drugs to the whole body. Toxicity of the drug limits the maximum dose achievable within a tumor or leads to unacceptable side effects [Avgeropoulos 1999, Groothuis 2000, Jain 2001]. Due to these complications, implantable delivery systems have gained special attention to control the release of drugs and localize treatments in specific targeted sites. These local delivery systems provide the opportunity for improving both the efficacy and safety for cancer treatment [Ewend 1996, Olivi 2003, Walter 1994]. Toxic side effects attributed to high drug concentrations can be minimized because the drug is delivered locally, rather than systemically. However, these implantable delivery systems require surgery near the target sites, which leads to additional costs and surgical pains [Avgeropoulos 1999, Westphal 2003].

In recent years, the injection of gel forming solutions has been considered a promising method to deliver drugs at target areas locally. Injectable hydrogels have several advantages over the common approach for drug delivery systems [Gutowska 2001, Hoemann 2005, Lee 2001, Molinaro 2002, Qu 1999, Roughley 2006, Ruel-Gariepy 2000, Ta 2008]. Their initial liquid formulation can flow easily and fill any target area shape. 
The gel can include a variety of therapeutic agents including hydrophobic drugs, growth factors, and cells. The polymer formulation can be stored at room temperature or below before the administration. Above all, there is no requirement for a surgical procedure for implantation. A therapeutic agent is delivered close to the targeted site by a simple injection of a liquid formulation, which undergoes thermal gelation at body temperature.

Chitosan based materials have been used in the area of drug delivery for sustained release of therapeutic agents [Bae 2006, Berrada 2005, Ruel-Gariepy 2004, Ta 2009]. Chitosan is an attractive biopolymer due to its biodegradability and biocompatibility. Recently, chitosan based injectable gels with various polyols such as glycerol, ethylene glycol, sorbitol, etc have gained significant attention because of their ability to deliver specific drugs at a target area [Back 1979, Chenite 2000, Iliescu 2008, Jarry 2002, Kokufuta 2005, Wu 2007]. These polyols maintain chitosan as a liquid. Polyols form a shield of water around chitosan chains in an acidic solution and maintain the solubility of chitosan at higher $\mathrm{pH}$ values at lower temperatures [Berger 2004, Jarry 2002 , Kokufuta 2005]. Especially, $\beta$-glycerophosphate ( $\beta$-GP) has attracted a lot of attention in forming thermo-sensitive chitosan based gel delivery vehicles [Chenite 2001, Cho 2005, 2006, Crompton 2007, Hoemann 2007, Wu 2006].

Several interactions reported during the gelation process are the loss of electrostatic repulsion, hydrogen bonding, and increased hydrophobic interactions. The addition of disodium $\beta$-GP into chitosan solution increases the $\mathrm{pH}$, thereby reducing the electrostatic repulsion between chitosan chains. This reaction, in turn, induces an increase in chitosan interchain hydrogen bonding. Raising the temperature strengthens the hydrophobic interactions between chitosan chains and induces gelation. The mechanism may be because the hydration water molecules hydrogen bonded to chitosan chains are released with increasing temperature. This would allow increased hydrophobic interactions between chitosan chains [Berger 2004, Cho 2005]. In order to form the gel network at body temperature within physiologically acceptable $\mathrm{pH}$ range, it is necessary to include high concentration of $\beta-G P$, which induces toxicity. To improve biocompatibility of this system, several researches have used low concentration of inorganic phosphate salts or added cross-linkers such as glyoxal and hydroxyethyl cellulose, which are responsible for the solidification of chitosan based hydrogels with low concentrations of $\beta$-GP [Crompton 2007, Hoemann 2007, Nair 2007, Roughley 2006].

In the present study, as shown in Figure 5.1, a novel method to produce a heatinduced chitosan gel is described which minimizes $\beta$-GP toxicity. Acidic chitosan solution is dialyzed to prior to adding $\beta$-GP reducing the amount of $\beta$-GP needed which may lead to biocompatibility. To test the vehicle for drug delivery, ellagic acid is added to the chitosan solution. Ellagic acid shows anti-carcinogenic properties via cell cycle arrest, induction of apoptosis, and inhibition of tumor formation and growth. In the previous studies [Kim 2009], it was found that a chitosan film delivery system loaded with ellagic acid could induce apoptotic cancer cell death in an ellagic acid concentration dependent manner in vitro and in vivo. The objective of this study was to develop and characterize a thermo-sensitive $\mathrm{Ch} / \beta$-GP gel and evaluate its anti-tumor effect on cancer cells (human U87 glioblastomas and rat C6 glioma cells) when loaded with ellagic acid in vitro. 


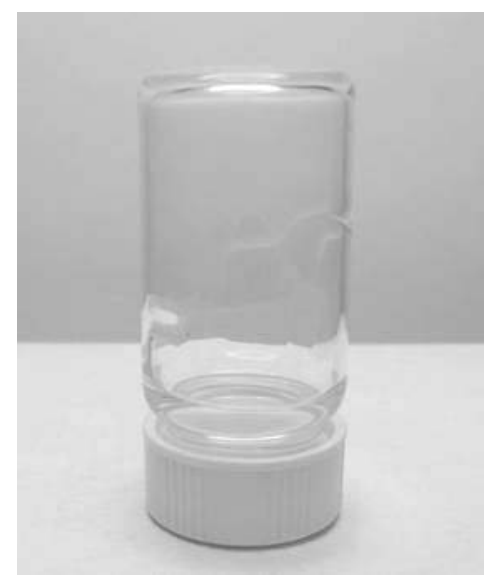

(a)

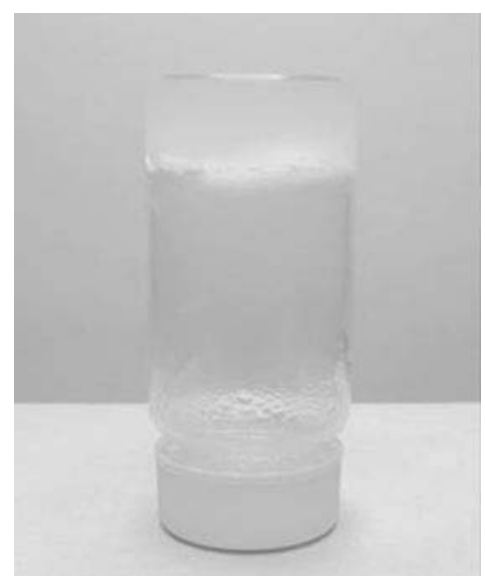

(b)

Figure 5.1 Photograph showing the thermal gelation of the chitosan/ $\beta$-GP solutions which were placed (a) at $5^{\circ} \mathrm{C}$ and (b) at $37^{\circ} \mathrm{C}$ for 30 minutes. $5 \mathrm{~mL}$ of chitosan solution contained $0.088 \mathrm{M}$ of $\beta$-GP solution and the final $\mathrm{pH}$ was 7.23.

\subsection{Materials and Methods}

\subsubsection{Materials}

The chitosan was purchased from Sigma Aldrich in high molecular weight $(\geq 310$ $\mathrm{kDa}$ ) with an approximately $75 \%$ or greater degree of deacetylation. The ellagic acid and disodium $\beta$-GP (glycerol 2-phosphate disodium salt hydrate; cell culture grade) were also obtained from Sigma Aldrich. All other chemicals were reagent grade and used as received. Fetal bovine serum (FBS), Trypsin-EDTA, L-Glutamine, AntibioticAntimycotic, Phosphate buffered saline (PBS), Ham's F12K medium, horse serum, and Dulbecco's Modified Eagle Medium (DMEM) were obtained from Gibco ${ }^{\mathrm{TM}}$. The U87 human glioblastoma, HS68 human newborn fibroblasts (passage 12) and C6 rat glioma cell lines were cultured as per American Type Culture Collection (ATCC) instructions.

\subsubsection{Preparation of the Chitosan / $\beta$-Glycerophosphate Solution: Ch/ $\beta-G P$}

A $1.5 \%(\mathrm{w} / \mathrm{v})$ chitosan solution was prepared by stirring powdered chitosan in $0.75 \%(\mathrm{v} / \mathrm{v})$ aqueous acetic acid at room temperature overnight. The insoluble particles in the chitosan solution were removed by filtration. A $50 \%(\mathrm{w} / \mathrm{v}) \beta-\mathrm{GP}$ solution was prepared in distilled water and sterilized using PES syringe filters with $0.22 \mu \mathrm{m}$ pore size (MillexTM, MA) and stored at $4^{\circ} \mathrm{C} .50 \mathrm{~mL}$ of chitosan solution was dialyzed at room temperature against 1 liter of distilled water for 6 days with daily changes of water $(1 \mathrm{~L})$ 
in an $8 \mathrm{kDa}$ cutoff dialysis membrane to reduce the acetic acid content. The final $\mathrm{pH}$ value of the chitosan solution was 6.17 . The dialyzed chitosan solution was autoclaved at $121^{\circ} \mathrm{C}$ for 20 minutes, cooled down to room temperature, and stored at $4^{\circ} \mathrm{C}$. Sterilized, ice-cold $\beta$-GP solution $(2.31 \mathrm{M})$ was added drop by drop to the chitosan solution under stirring conditions in an ice bath. The final concentration of $\beta$-GP in the chitosan solution ranged from $0.045 \mathrm{M}$ to $0.660 \mathrm{M}$, and the final $\mathrm{pH}$ value ranged from 6.9 to 7.2. The dialyzed solutions were labeled DCh/ $\beta$-GP. The DCh $/ \beta$-GP solutions were placed into 50 $\mathrm{mL}$ tubes, sealed, and stored at $4^{\circ} \mathrm{C}$. In addition, undialyzed chitosan solutions (initial $\mathrm{pH}$ 3.97 ) were prepared, mixed with a cold $\beta$-GP solution, and stored at $4^{\circ} \mathrm{C}$. The final $\mathrm{pH}$ value of $\mathrm{Ch} / \beta$-GP solution ranged from 6.9 to 7.2 , and the final $\beta$-GP concentrations ranged from $1.027 \mathrm{M}$ to $1.155 \mathrm{M}$.

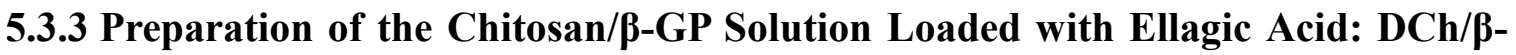 GP/EA}

Ellagic acid solutions in distilled water $(0.1$ or $1 \%(\mathrm{w} / \mathrm{v}))$ were prepared. The powdered ellagic acid was dissolved into distilled water by adding several drops of $0.1 \mathrm{M}$ $\mathrm{NaOH}$, with constant stirring at room temperature overnight. The $1.5 \%(\mathrm{w} / \mathrm{v})$ chitosan solutions were then mixed with the ellagic acid solutions at a chitosan to ellagic acid volume ratio of $1: 1$. The mixed chitosan/ellagic acid solutions were agitated at $40^{\circ} \mathrm{C}$ for 2 hours and then neutralized over 3 or 4 days using a dialysis membrane. The chitosan/ellagic acid solutions were autoclaved at $121^{\circ} \mathrm{C}$ for 20 minutes, cooled down to room temperature, and stored at $4^{\circ} \mathrm{C}$. Finally, they were mixed with the $\beta$-GP solution as described above.

\subsubsection{Characterization of the Chitosan/ $\beta$-GP Gel}

\subsubsection{FTIR Spectra}

To characterize the chemical structure of the $\mathrm{DCh} / \beta$-GP solution at $5^{\circ} \mathrm{C}$ and $37^{\circ} \mathrm{C}$, FTIR spectra were obtained using a Nicolet FTIR Infrared Microscope coupled to a PC with analysis software. Samples were placed in a liquid cell assembled holder in the IR laser beam. All samples were chilled in an ice bath until they were incubated at $37^{\circ} \mathrm{C}$. FTIR spectra of samples at $5^{\circ} \mathrm{C}$ and $37^{\circ} \mathrm{C}$ were obtained and compared. All spectra were recorded by transmittance mode (100 times scanning, $\left.650-4000 \mathrm{~cm}^{-1}\right)$.

\subsubsection{Scanning Electron Microscopy}

The shapes and surface morphology of DCh/ $\beta$-GP gel and DCh/ $\beta$-GP/EA gel were observed by field emission scanning electron microscope (JEOL, JSM-633OF). The samples were prepared at $37^{\circ} \mathrm{C}$ for 3 hours and then dipped into distilled water at $37^{\circ} \mathrm{C}$ for 1 day. Next, they were lypholized overnight (Freezone, LABCONCO). The samples were fixed with conductive tape on a metal stub, coated with a carbon rod under vacuum, and examined at a $15 \mathrm{kV}$ accelerating voltage with a working distance of $17 \mathrm{~mm}$. 


\subsubsection{Rheological Analysis}

Rheological measurement was performed with an AR 1000 rheometer (TA instruments) equipped with a coquette geometry, which required about $1 \mathrm{~mL}$ of the solution per sample. The top of geometry was filled with $1.5 \mathrm{~mL}$ of distilled water to create a humidified chamber. The chitosan solutions containing $1.027 \mathrm{M}$ (final $\mathrm{pH}$ 6.97) or $1.155 \mathrm{M}$ (final $\mathrm{pH}$ 7.17) of $\beta$-GP and the dialyzed chitosan solutions containing 0.045 $\mathrm{M}$ (final $\mathrm{pH}$ 6.98) or $0.088 \mathrm{M}$ (final $\mathrm{pH}$ 7.23) of $\beta$-GP were introduced to the rheometer. During the gelation process, the rheological properties of samples were measured with a temperature range of 10 to $50^{\circ} \mathrm{C}$. The changes in the elastic (storage) modulus $\left(G^{\prime}\right)$ and the viscous (loss) modulus $\left(G^{\prime \prime}\right)$ were recorded as function of temperature at a fixed frequency of $1 \mathrm{~Hz}$. The phase lag $(\delta)$ and complex viscosity $\left(\eta^{*}\right)$ were taken to determine the gelation temperature at which the elastic modulus $\left(G^{\prime}\right)$ and the viscous modulus $\left(G^{\prime \prime}\right)$ were equivalent in value $\left(\delta=45^{\circ}\right)$. The temperature was varied with a constant heating rate of $1{ }^{\circ} \mathrm{C} / \mathrm{min}$

\subsubsection{Gelation Time Determination}

In order to determine the gelation time, the dialyzed chitosan solution containing $0.045 \mathrm{M}$ (final $\mathrm{pH} 6.98$ ) or $0.088 \mathrm{M}$ (final $\mathrm{pH}$ 7.23) of $\beta$-GP were prepared. The $5 \mathrm{~mL}$ of each sample was tested in glass vials $(27 \times 61 \mathrm{~mm}$, Wheaton Glass). Gelation time was measured as a function of time at constant temperatures of $24^{\circ} \mathrm{C}, 37^{\circ} \mathrm{C}, 50^{\circ} \mathrm{C}$, and $70^{\circ} \mathrm{C}$ in a water bath. The test tube inverting method was used to determine the gelation time [Nair 2007, Ta 2009]. The samples were incubated in a water bath at the predetermined temperatures, and time measurements were initiated. The flowability of the samples was observed every 30 seconds by tilting the tubes. The time at which flowing of the samples stopped was taken as the gelation time and the values were recorded.

\subsubsection{In vitro Release Studies}

Ellagic acid release from the DCh/ $\beta$-GP gel was quantified over time by an in vitro release assay. Dialyzed chitosan solutions containing $0.088 \mathrm{M}$ of $\beta$-GP solution were loaded with $5.5 \mathrm{mg}(\mathrm{DCh} / \beta-\mathrm{GP} / \mathrm{EA} 0.1)$ or $10 \mathrm{mg}(\mathrm{DCh} / \beta-\mathrm{GP} / \mathrm{EA} 1)$ of ellagic acid. $1 \mathrm{~mL}$ of each DCh/ $/$-GP/EA solution was allowed to completely become a gel in an incubator at $37^{\circ} \mathrm{C}$ for 3 hours and placed in containers containing $6 \mathrm{~mL}$ of distilled water at $37^{\circ} \mathrm{C}$. At designated time points $(1,3,7,14$, and 21 days), $500 \mu \mathrm{L}$ aliquots of the release medium were sampled and the same amount of fresh distilled water was added into the containers. In addition, release of ellagic acid in the presence of lysozyme $(1 \mathrm{mg} / \mathrm{mL})$ was also determined in order to examine the effect of an enzyme on the release profile. In the collected fractions, the cumulative amount of ellagic acid released as a function of time was determined by a UV spectrophotometer at $260 \mathrm{~nm}$. 


\subsubsection{In vitro Enzymatic Degradation}

Dialyzed chitosan solutions containing $0.088 \mathrm{M}$ of $\beta$-GP solution were loaded with $5.5 \mathrm{mg}(\mathrm{DCh} / \beta-\mathrm{GP} / \mathrm{EA} 0.1)$ or $10 \mathrm{mg}(\mathrm{DCh} / \beta-\mathrm{GP} / \mathrm{EA} 1)$ of ellagic acid. $1 \mathrm{~mL}$ of each $\mathrm{DCh} / \beta$-GP/EA solution was allowed to become a gel in an incubator at $37^{\circ} \mathrm{C}$ for 3 hours and digested in 12-well plates containing phosphate buffer saline (PBS, pH 6) with 1 $\mathrm{mg} / \mathrm{mL}$ lysozyme (egg-white, Fisher Scientific, Fair Lawn, NJ) at $37^{\circ} \mathrm{C}$. After the predetermined time points, $500 \mu \mathrm{L}$ aliquots of medium from each sample were collected and the same amount of fresh lysozyme $(1 \mathrm{mg} / \mathrm{mL})$ was added. A ninhydrin assay was performed to determine the amount of free amino groups in the incubation medium collected for $1,3,7,14$, and 21 days. After heating with ninhydrin at $95^{\circ} \mathrm{C}$ for 10 minutes, the light absorbance at $550 \mathrm{~nm}$ was recorded using a micro plate reader (Molecular Device Vmax kinetic). Glycine (Fisher Scientific, Fair Lawn, NJ) was used as an amino acid nitrogen standard at various known concentrations. Enzymatic degradation rate was determined by measuring the increase of $\mathrm{N}$-glucosamine units in the incubation medium.

\subsubsection{Cell Culture}

U87 human glioblastoma and HS68 human newborn fibroblast (passage 12) cell lines were grown and maintained in DMEM media with $10 \%$ FBS, $1 \%$ antibiotic/antimycotic mixture, $5 \mathrm{~mL}$ of L-glutamine $(200 \mathrm{mM})$, and sodium pyruvate. The C6 rat glioma cell line was grown and maintained in Ham's F12K medium with 2.5\% FBS, $15 \%$ horse serum, $2 \mathrm{mM}$ L-glutamine adjusted to contain $1.5 \mathrm{~g} / \mathrm{L}$ sodium bicarbonate, and $1 \%$ antibiotic/antimycotic mixture. The cells were cultured in an incubator supplied with $5 \% \mathrm{CO}_{2}$ at $37^{\circ} \mathrm{C}$. The culture medium was changed every 3 days.

\subsubsection{Cytotoxicity of $\beta$-GP Solution on Cell Culture}

To investigate the toxic effect of the $\beta$-GP solution on cell culture, viability of human HS68 newborn fibroblasts was investigated with a MTS assay and the cell morphology was observed by microscope (Nikon ECLIPSE TE-2000-U). A $\beta$-GP solution $(2.31 \mathrm{M})$ was prepared and sterilized using a PES syringe filter with $0.22 \mu \mathrm{m}$ pore size (MillexTM, MA). A sterilized $\beta$-GP solution was added to cell culture media, and the final concentrations of $\beta$-GP in the media were $0,45,88,131,210,385,533$, and 660 $\mathrm{mM}$ (dilution of $\beta$-GP solution, originally $2.31 \mathrm{M}$ ). The cells were seeded on each well of 48 -well plates at a density of 30,000 cells per well in $300 \mu \mathrm{L}$ of culture medium. The cells were cultured without medium exchange for 4,24 , and 72 hours. At the end of the incubation period, the cell viability was determined with the Cell Titer 96AQueous One Solution Cell Proliferation Assay (MTS assay, Promega Corp.). $60 \mu \mathrm{L}$ of Cell Titer 96AQueous One Solution was added into each well of the 48-well plates, containing the samples in $300 \mu \mathrm{L}$ of culture medium. Following an incubation period of 2 hours at $37^{\circ} \mathrm{C}$ $5 \% \mathrm{CO}_{2}, 100 \mu \mathrm{L}$ of solution in each well of the 48 -well plates was transferred into 96well assay plates and the light absorbance at $490 \mathrm{~nm}$ was recorded using a micro plate reader (Molecular Device Vmax kinetic). Prior to the MTS assay, the cell morphology was documented using an inverted bright field (BF) microscope (Nikon ECLIPSE TE- 
2000U) and processed using MetaVue software.

\subsubsection{Cytotoxicity of Chitosan/ $\beta$-GP Delivery System on Cell Culture}

To investigate the biological effect of chitosan/ $\beta$-GP gels on cells, viability of human HS68 newborn fibroblasts was examined by a MTS assay, and cell morphology was observed by microscope (Nikon ECLIPSE TE-2000-U). Indirect culture via BD BioCoat ${ }^{\mathrm{TM}}$ Control Cell Culture inserts (12 well formats) was used to evaluate the effect of the chitosan/ $\beta$-GP gels on cell viability through cell culture media. The insert contains a 0.45 micron pore size PET membrane with a thin layer of basement membrane which prevents cells from migrating through the membrane and allows only the culture medium through the PET membrane. Two different chitosan/ $\beta$-GP gels were prepared. A dialyzed chitosan solution ( $\mathrm{pH}$ 6.17) was mixed with $0.088 \mathrm{M}$ of $\beta$-GP solution, while a chitosan solution (pH 3.96) was mixed with $1.155 \mathrm{M}$ of $\beta$-GP solution. The final $\mathrm{pH}$ values of the solutions were 7.23 and 7.17 , respectively. $1 \mathrm{~mL}$ of each chitosan/ $\beta$-GP solution was placed into the bottom of well plates and incubated at $37^{\circ} \mathrm{C}$ for 1 hour prior to cell culture. $1.7 \mathrm{~mL}$ of the cell culture medium was added into each well of the 12-well plates with inserts, which were allowed to hydrate for 2 hours at $37^{\circ} \mathrm{C}$. The cells were then seeded at a density of 60,000 cells per well in the upper chamber with $0.3 \mathrm{~mL}$ of culture medium. After incubation for 4, 24, and 72 hours, the number of viable cells was determined using a Cell Titer 96AQueous One Solution assay according to the manufacturer's instructions. Photomicrographs of cells were also documented during cell culture using a microscope and processed using MetaVue software. Cells were seeded on the chitosan/ $\beta$-GP gels in 24-well plates at a density of 60,000 cells per well and incubated with $500 \mu \mathrm{L}$ of culture media for 3 days, after which the viable cells were stained with the calcein AM and then cell morphology was observed.

\subsubsection{In vitro Anti-Tumor Activity}

To evaluate the anti-tumor effect of $\mathrm{Ch} / \beta$-GP gels loaded with ellagic acid on the two cancer cell lines, the number of viable cells (human U87 glioblastoma and rat C6 glioma) was measured by a MTS assay. Indirect culture via BD BioCoat ${ }^{\mathrm{TM}}$ Control Cell Culture inserts (12 well formats) was used to evaluate the anti-proliferative effect of $\mathrm{Ch} / \beta$-GP/EA gels on cancer cells via cell culture media. The dialyzed chitosan solutions containing $0.088 \mathrm{M}$ of $\beta$-GP solution were loaded with $5.5 \mathrm{mg}(\mathrm{DCh} / \beta$-GP/EA0.1) or 10 $\mathrm{mg}(\mathrm{DCh} / \beta-\mathrm{GP} / \mathrm{EA} 1)$ of ellagic acid. $1 \mathrm{~mL}$ of each $\mathrm{DCh} / \beta$-GP/EA solution was placed into the bottom of each well plate and incubated for 1 hour prior to cell culture to become a gel. $1.7 \mathrm{~mL}$ of the cell culture medium was added into each well of the 12-well plate, and cells were then seeded at a density of 60,000 cells per well in the upper chamber with $0.3 \mathrm{~mL}$ of the culture medium. After incubation for 4,24 , and 72 hours, the number of viable cells was determined using the Cell Titer 96AQueous One Solution assay as described above. 


\subsubsection{Statistical Analysis}

All data are presented as mean \pm standard deviation. Significant differences in release profile, degradation rate, and cell activity were analyzed by one-way ANOVA test. The differences in groups and experimental time points at any time were considered significant if $p<0.05$.

\subsection{Results}

\subsubsection{FTIR Spectra}

The FTIR spectra obtained from the chitosan/ $\beta$-GP solution at $5^{\circ} \mathrm{C}$ and $37^{\circ} \mathrm{C}$ are shown in Figure 5.2. The protonation of the phosphate groups induced a reduction of the stretching of phosphate group $\left(-\mathrm{PO}_{4}{ }^{2-}\right)$ at $978 \mathrm{~cm}^{-1}$ with increasing temperature [Arai 2001, Elzinga 2007]. The peaks of the diprotonated phosphate group $\left(-\mathrm{H}_{2} \mathrm{PO}_{4}\right)$ at $37^{\circ} \mathrm{C}$ were shown at the range of 1146-1159 $\mathrm{cm}^{-1}$, indicating the nonprotonated phosphate group $\left(-\mathrm{PO}_{4}{ }^{2-}\right)$ accepted protons $\left(\mathrm{H}^{+}\right)$[Arai 2001, Elzinga 2007]. A broad band at 1206$1250 \mathrm{~cm}^{-1}$ is assigned to the POH bending both in the chitosan/ $\beta$-GP solution at $5^{\circ} \mathrm{C}$ and $37^{\circ} \mathrm{C}$ [Elzinga 2007]. The intensity of the peaks in the range from 1620 to $1722 \mathrm{~cm}^{-1}$, which are attributed to the $\mathrm{C}=\mathrm{O}$ stretching of amide I bonds of chitosan, were changed with increasing temperature. In addition, there were significant changes in spectrum shape at 1554,1512 , and $1427 \mathrm{~cm}^{-1}$, which are representative of the $\mathrm{N}-\mathrm{H}$ bending vibrations of amide II bond of the chitosan with increasing temperature [Tien 2003, Wang 2004].

\subsubsection{Scanning Electron Microscopy}

Figure 5.3 displays the SEM micrographs of the DCh/ $\beta$-GP gels which were lyophilyzed overnight before the examination. The morphological structure of the $\mathrm{DCh} / \beta$ GP gel and the DCh/ $\beta$-GP/EA gel was observed after dipping into distilled water for 1 day. The DCh/ $\beta$-GP gel (Figure $5.3 \mathrm{a}$ ) showed irregular porous structure while the surface morphology of the $\mathrm{DCh} / \beta$-GP/EA (Figure $5.3 \mathrm{~b}$ ) became rougher and more textured.

\subsubsection{Rheological Analysis}

In order to determine the gelation temperature of the chitosan/ $\beta$-GP solution, an AR 1000 rheometer (TA instruments) was used to measure the storage (elastic) modulus $\left(G^{\prime}\right)$, loss (viscous) modulus $\left(G^{\prime \prime}\right)$, phase angle $(\delta)$ and complex viscosity $\left(\eta^{*}\right)$. With increasing temperature from $10^{\circ} \mathrm{C}$ to $50^{\circ} \mathrm{C}$, all samples had a rapid increase of the storage modulus $\left(G^{\prime}\right)$, showing the phase transition of the chitosan/ $\beta$-GP solution to the chitosan $/ \beta$-GP gel around $37^{\circ} \mathrm{C}$. The greater growth rate of the storage modulus $\left(G^{\prime}\right)$ compared with the loss modulus $(G$ ") indicated that development of the gel structure 


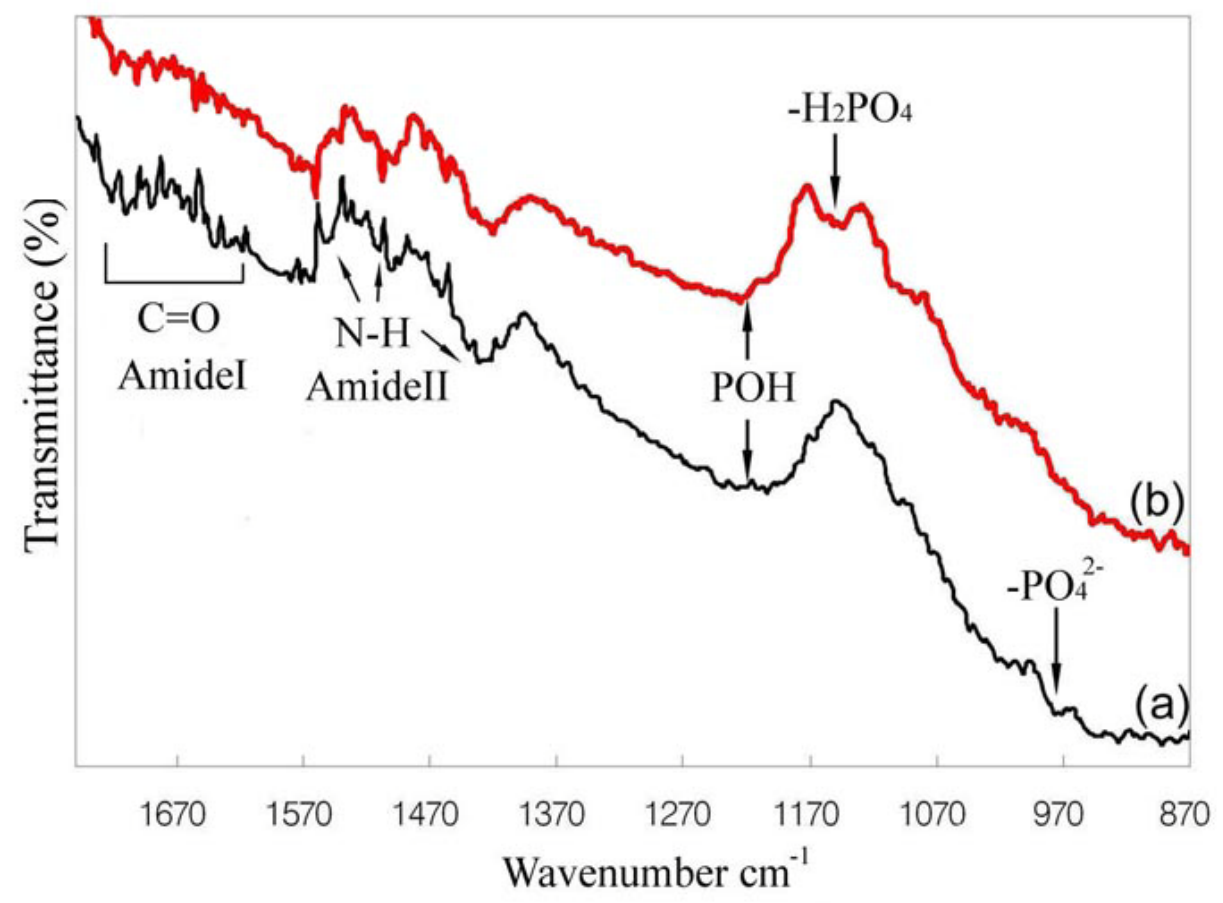

Figure 5.2 FTIR spectra of the chitosan/ $\beta$-GP solution. (a) at $5^{\circ} \mathrm{C}$ and (b) $37^{\circ} \mathrm{C}$. The chitosan solution contained $0.088 \mathrm{M}$ of $\beta$-GP solution and its final $\mathrm{pH}$ was 7.23. 


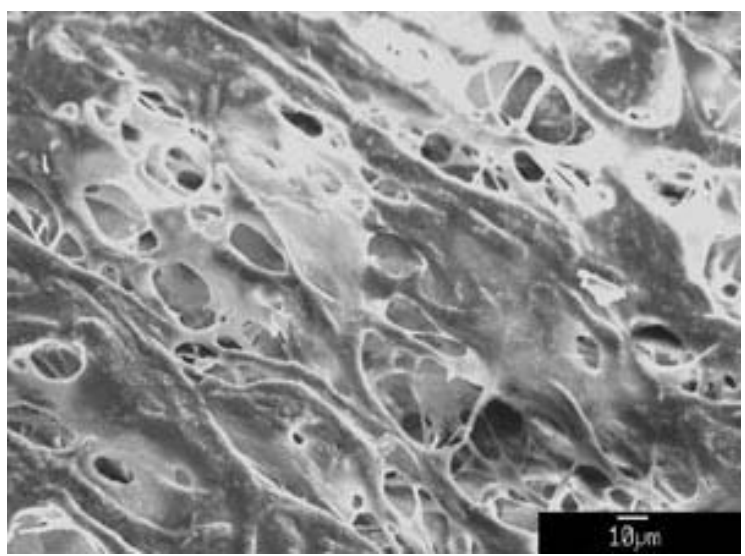

(a)

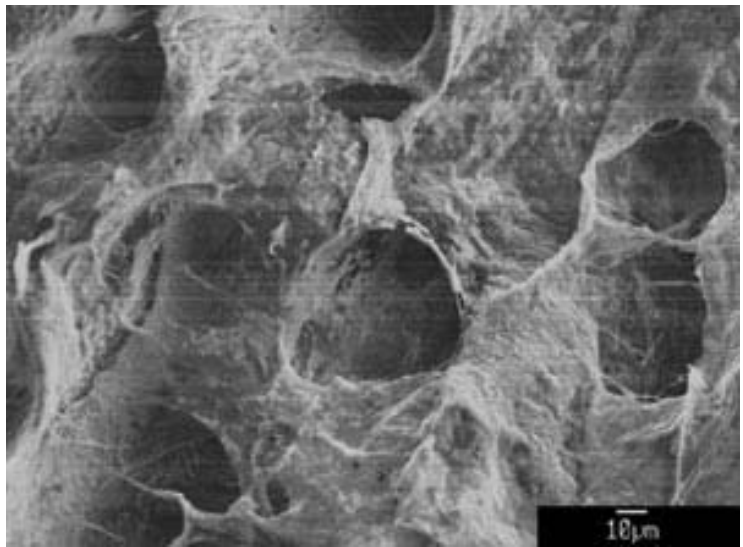

(b)

Figure 5.3 SEM micrographs on the surface of the chitosan $/ \beta-G P$ gels. (a) $D C h / \beta-G P$ gel and (b) $D C h / \beta-G P / E A$ gel. The samples were prepared by dipping chitosan $/ \beta$-GP gels in distilled water at $37^{\circ} \mathrm{C}$ for 1 day, followed by lyophilization overnight $(\mathrm{MAG}=\times 500$ ). 
contributed to the increase in elasticity. Figure 5.4 shows the effect of the final $\mathrm{pH}$ value of the $\mathrm{DCh} / \beta$-GP solution on the gelation temperature.

The crossover of the storage modulus $\left(G^{\prime}\right)$ and the loss modulus ( $\left.G^{\prime \prime}\right)$ moved to the left, indicating that the gelation temperature was lowered with increasing final $\mathrm{pH}$ of the gel forming solution as shown in Figure 5.4a and b. The phase angle $(\delta)$ of a dialyzed chitosan solution containing $0.045 \mathrm{M}$ (final $\mathrm{pH} 6.98$ ) of $\beta$-GP and $0.088 \mathrm{M}$ (final $\mathrm{pH}$ 7.23) of $\beta$-GP decreased gradually as the temperature increased. The phase angle reached $45^{\circ}$ where $G^{\prime}=G$ " at which the temperature was $33^{\circ} \mathrm{C}$ for $\mathrm{pH} 6.98$ and $26^{\circ} \mathrm{C}$ for $\mathrm{pH} 7.23$, respectively. This result indicated that the storage modulus $\left(G^{\prime}\right)$ began to increase at lower temperature with $0.088 \mathrm{M}$ of $\beta$-GP (final $\mathrm{pH} 7.23$ ) compared with $0.045 \mathrm{M}$ of $\beta$-GP (final $\mathrm{pH}$ 6.98). The complex viscosity $\left(\eta^{*}\right)$ of a chitosan solution containing $0.088 \mathrm{M}$ of $\beta$-GP also showed a sharp rise at lower temperature compared with $0.045 \mathrm{M}$ of $\beta$-GP as shown in Figure 5.4c and d.

The gelation temperature of chitosan solutions containing high concentrations of $\beta$-GP (1.027 $\mathrm{M}$ and $1.155 \mathrm{M}$ ) was also investigated (Figure 5.5). Their final $\mathrm{pH}$ values were 6.97 and 7.17 , respectively. The crossover point and $45^{\circ}$ phase angle (where $G^{\prime}=G^{\prime \prime}$ ) came at lower temperature of $34^{\circ} \mathrm{C}$ with $1.155 \mathrm{M}$ of $\beta$-GP than the crossover point of $37^{\circ} \mathrm{C}$ with $1.027 \mathrm{M}$ of $\beta-\mathrm{GP}$ as shown in Figure $5.5 \mathrm{a}$ and $\mathrm{b}$. In comparison with the chitosan solution containing lower concentrations of $\beta$-GP $(0.045 \mathrm{M}$ or $0.088 \mathrm{M})$, the phase angle $(\delta)$ of the chitosan solution containing higher concentrations of $\beta$-GP (1.027 $\mathrm{M}$ or $1.155 \mathrm{M}$ ) decreased dramatically, rather than gradually. The results clearly demonstrated that chitosan solutions can undergo viscosity changes consistent with thermal gelation below body temperature after neutralization with either low concentrations of $\beta$-GP or high concentrations of $\beta$-GP. The gelation temperature was affected by the final $\mathrm{pH}$ of the chitosan $/ \beta$-GP solution.

\subsubsection{Gelation Time Determination}

In order to investigate the effect of the temperature on the gelation time, the fluidity of chitosan solutions were measured using the test tube inverting method. Figure 5.6 shows that the gelation time decreases exponentially with increasing temperature. In addition to the changes in flow capabilities, the samples became opaque as the samples formed a gel. The chitosan solution at $\mathrm{pH} 7.23$ containing $0.088 \mathrm{M}$ of $\beta$-GP showed faster gelation time $\left(150\right.$ seconds at $\left.37^{\circ} \mathrm{C}\right)$ compared with the chitosan solution containing $0.045 \mathrm{M}$ of $\beta$-GP $\left(330\right.$ seconds at $\left.37^{\circ} \mathrm{C}\right)$. Gel formation times were also different at $24^{\circ} \mathrm{C}$. At higher temperatures gel times became similar and were indistinguishable at $70^{\circ} \mathrm{C}$. The shortest gel time observed in this assay was 60 seconds.

\subsubsection{In vitro Release Studies}

In order to investigate the in vitro release rate of ellagic acid from the $\mathrm{DCh} / \beta$ GP/EA gels, the cumulative amount of ellagic acid released into the medium was 
Figure 5.4 The gelation temperature of the dialyzed chitosan solution. (a) $0.045 \mathrm{M}$ of $\beta$ GP (pH 6.98); (b) $0.088 \mathrm{M}$ of $\beta$-GP (pH 7.23) and the complex viscosity of the chitosan solution containing (c) $0.045 \mathrm{M}$ of $\beta$-GP; (d) $0.088 \mathrm{M}$ of $\beta$-GP. The storage modulus $\left(G^{\prime}\right)$ and the loss modulus $(G$ ") of the chitosan $/ \beta$-GP solution were evaluated with regard to the effect of the final $\mathrm{pH}$ on the gelation temperature. The effect of the final $\mathrm{pH}$ on the complex viscosity was also investigated as a function of temperature at $\omega=6.28 \mathrm{rad} / \mathrm{s}$ with $1^{\circ} \mathrm{C} / \mathrm{min}$. The elastic modulus $\left(G^{\prime}\right)$ and the viscous modulus $\left(G^{\prime \prime}\right)$ are equivalent in value at $\delta=45^{\circ}$. 


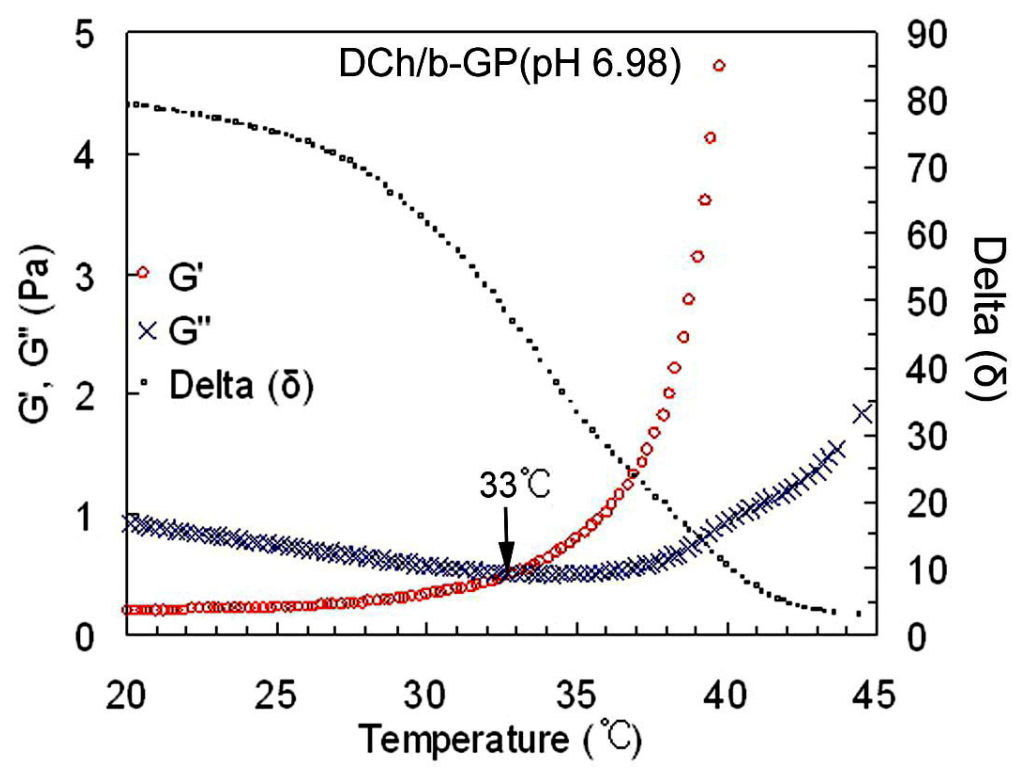

(a)

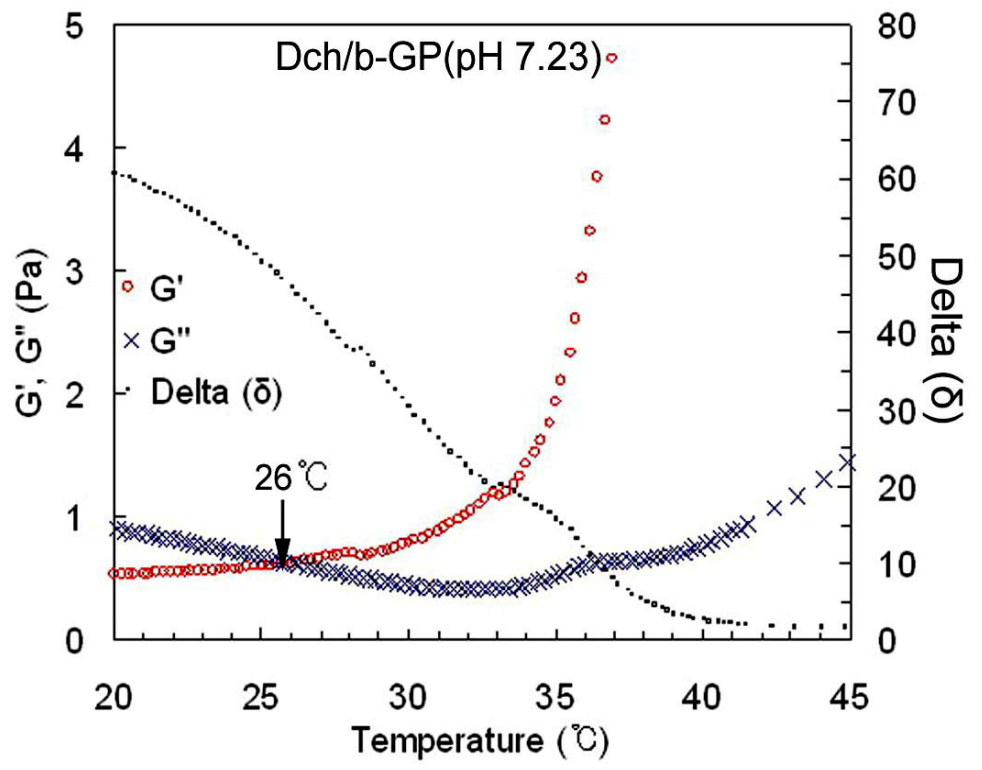

(b) 


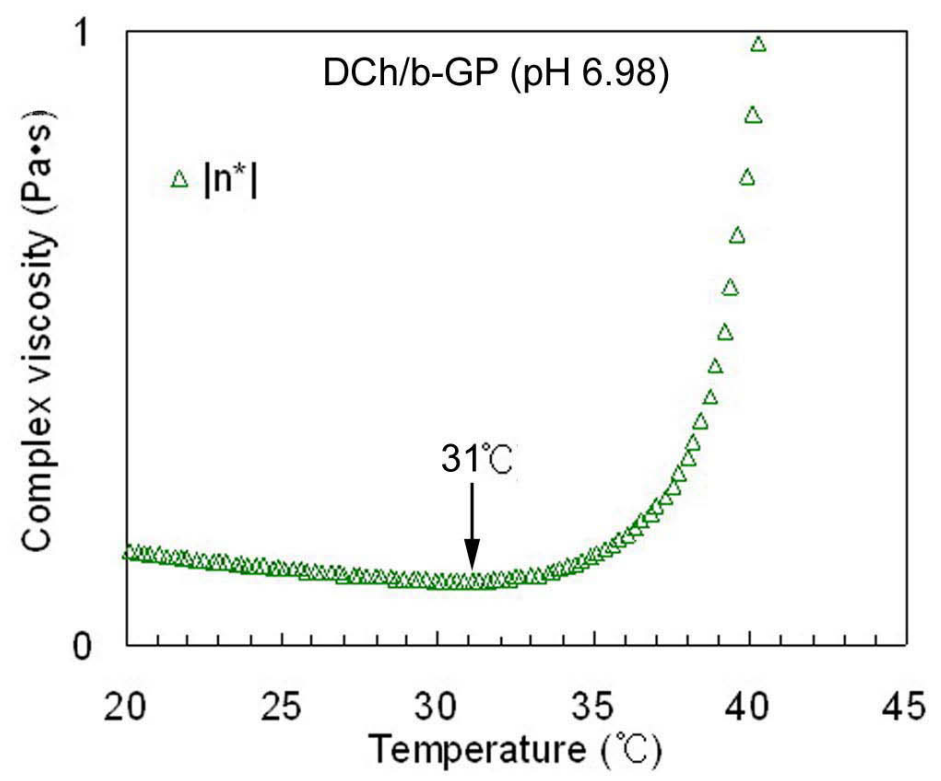

(c)

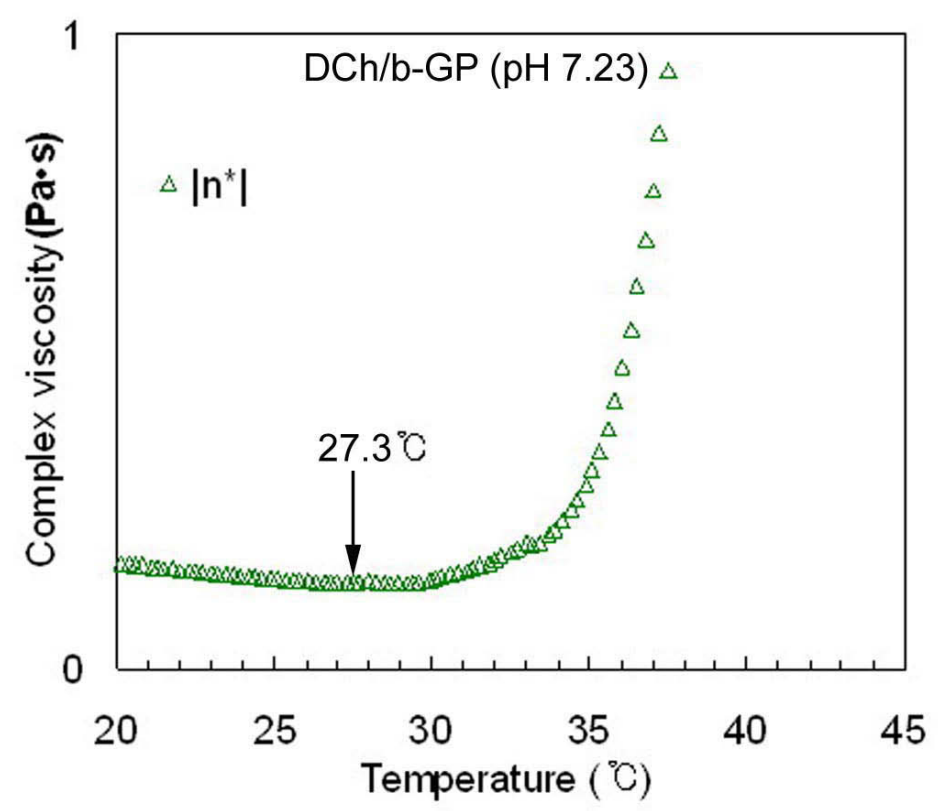

(d)

Figure 5.4 (continued). 
Figure 5.5 The gelation temperature of the undialyzed chitosan solution. (a) $1.027 \mathrm{M}$ of $\beta$-GP ( $\mathrm{pH}$ 6.97); (b) $1.155 \mathrm{M}$ of $\beta$-GP ( $\mathrm{pH} \mathrm{7.17)}$ and the complex viscosity of the undialyzed chitosan solution containing (c) $1.027 \mathrm{M}$ of $\beta$-GP; (d) $1.155 \mathrm{M}$ of $\beta$-GP. The storage modulus $\left(G^{\prime}\right)$ and the loss modulus $\left(G^{\prime \prime}\right)$ of the chitosan/ $\beta$-GP solution were evaluated with regard to the effect of the final $\mathrm{pH}$ on the gelation temperature. The effect of the final $\mathrm{pH}$ on the complex viscosity was also investigated as a function of temperature at $\omega=6.28 \mathrm{rad} / \mathrm{s}$ with $1^{\circ} \mathrm{C} / \mathrm{min}$. The elastic modulus $\left(G^{\prime}\right)$ and the viscous modulus $\left(G\right.$ ') are equivalent in value at $\delta=45^{\circ}$. 


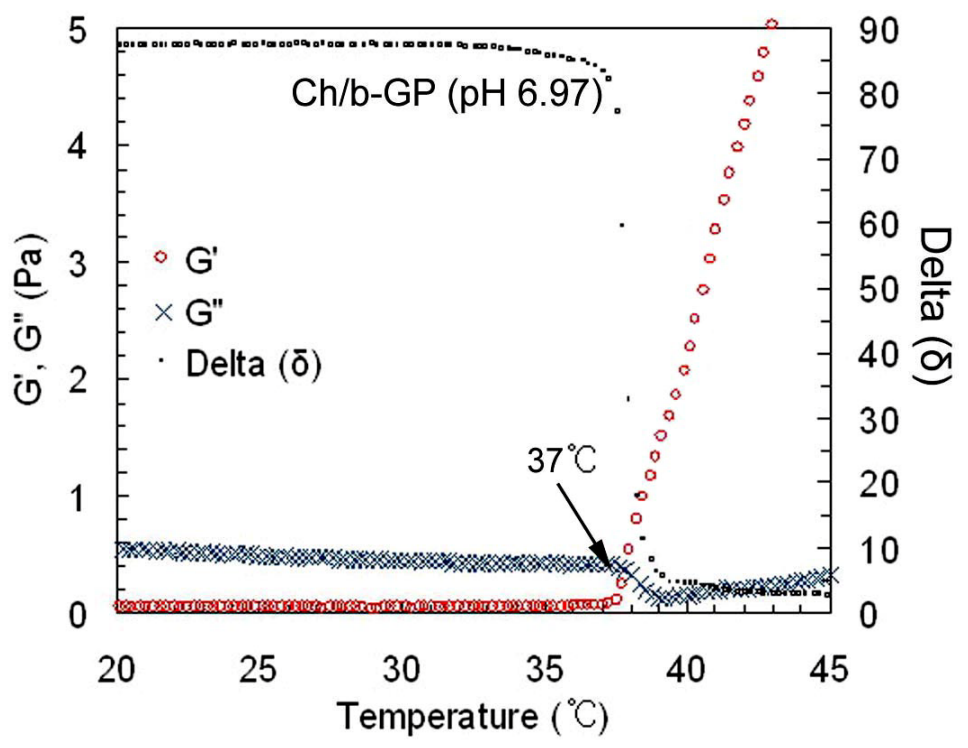

(a)

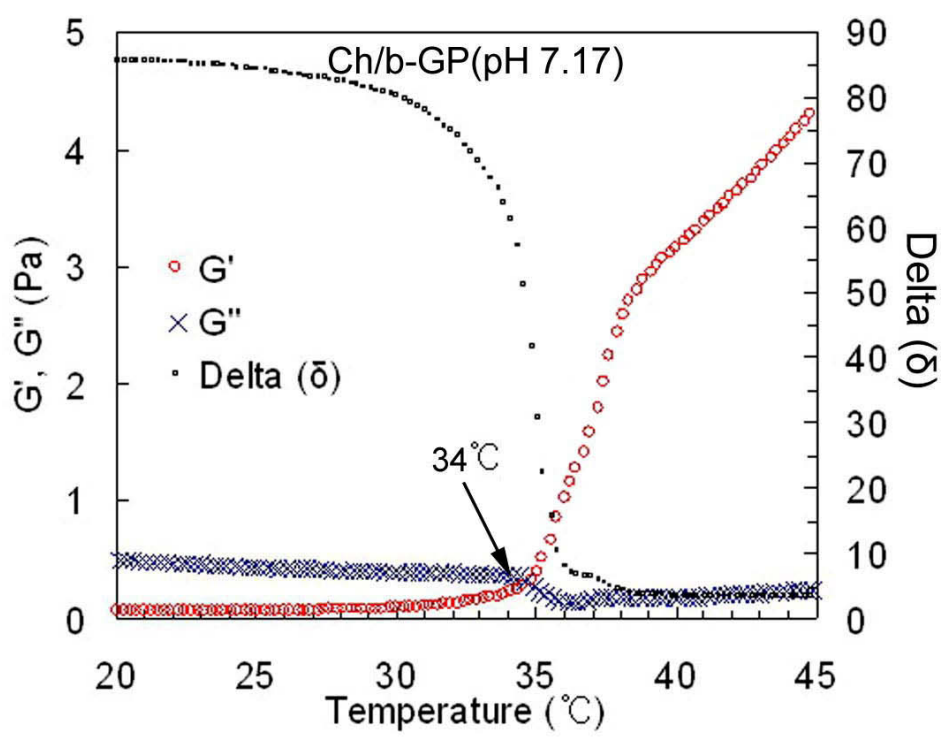

(b) 


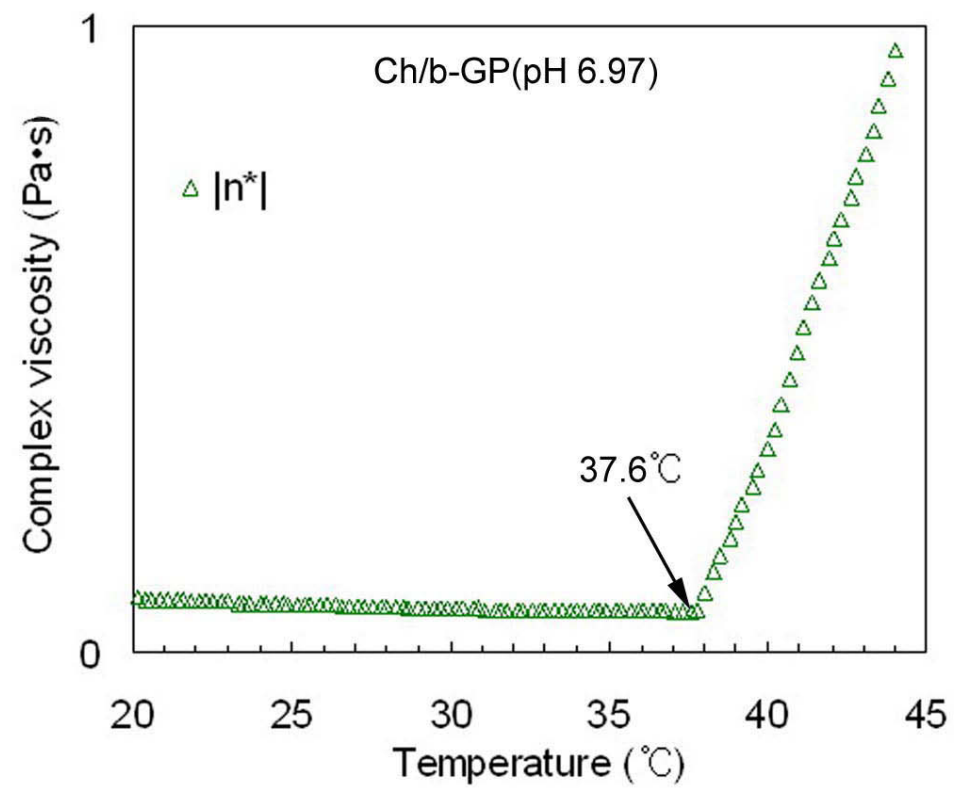

(c)

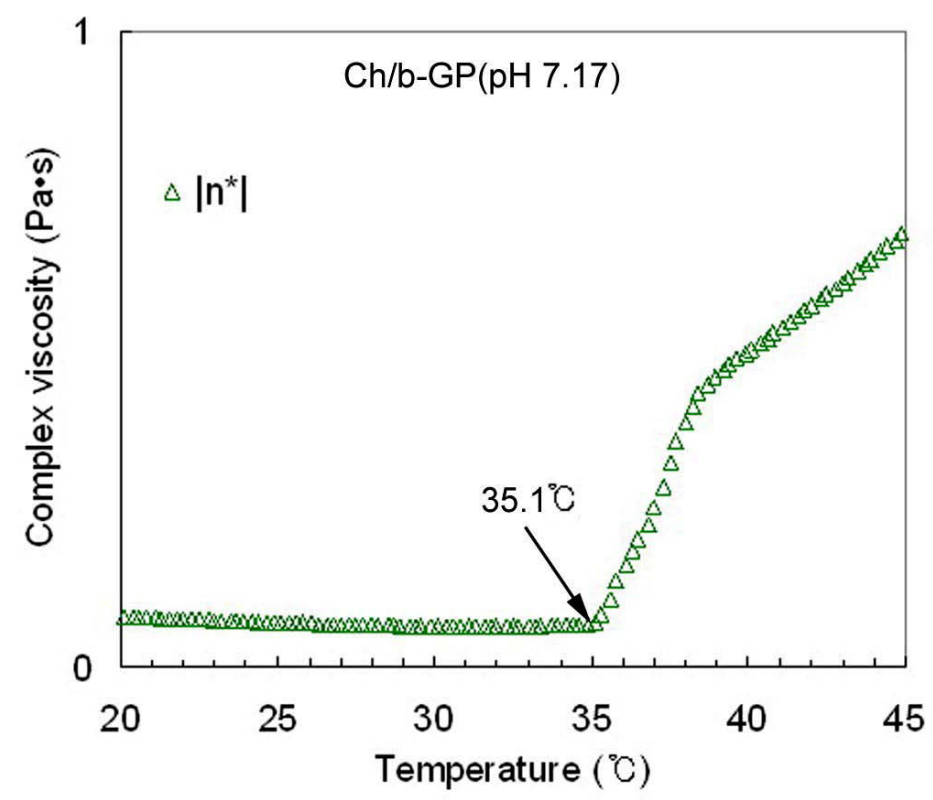

(d)

Figure 5.5 (continued) 


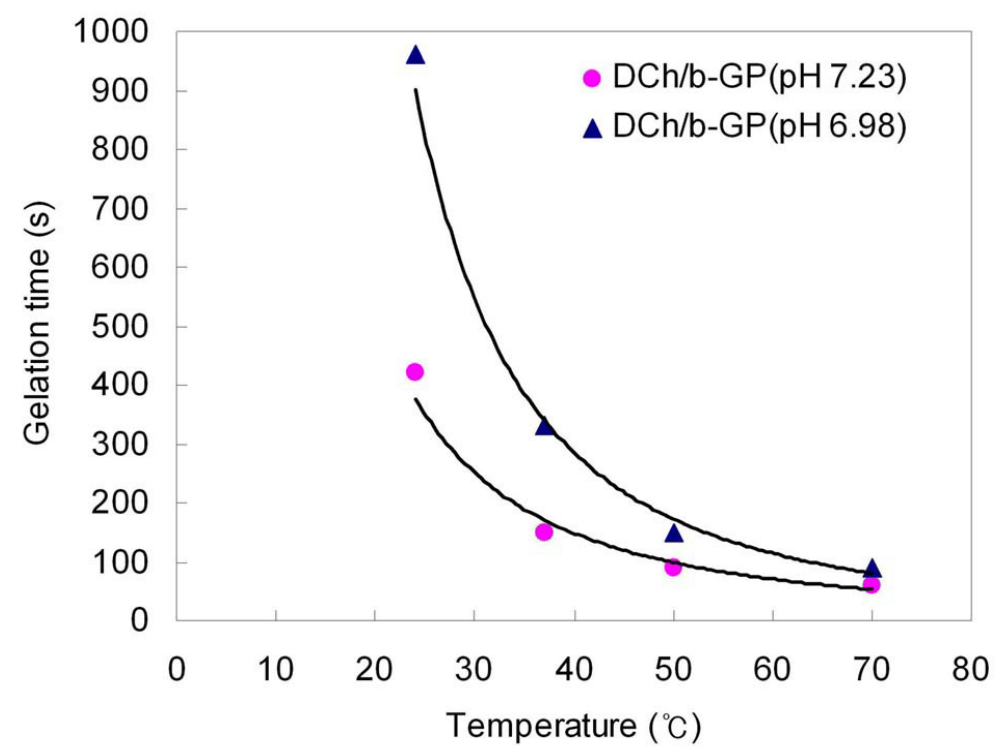

Figure 5.6 The gelation time of the DCh/ $\beta$-GP solution as a function of the temperature. The DCh/ $\beta$-GP solutions were prepared with different final $\mathrm{pHs}$ which were 6.98 and 7.23 , respectively. 
measured as a function of time. Figure 5.7 shows the cumulative release profiles of ellagic acid from the $\mathrm{DCh} / \beta-\mathrm{GP} / \mathrm{EA}$ gels.

The initial drug loadings significantly affected the release rate of ellagic acid from the DCh/ $\beta$-GP gels for 3 week incubation periods $(p<0.05)$. The chitosan $/ \beta-G P$ gels containing higher ellagic acid loading (DCh/ $\beta$-GP/EA1) released greater amounts of ellagic acid than lower ellagic acid loading (DCh/ $\beta$-GP/EA0.1). There was a sustained slow release of ellagic acid from the chitosan $/ \beta-\mathrm{GP}$ gels.

In addition, the effect of chitosan lytic enzymes on the release profiles of the chitosan/ $\beta$-GP gels was evaluated in the presence of lysozyme. The cumulative amount of ellagic acid released from $\mathrm{DCh} / \beta-\mathrm{GP} / \mathrm{EA}$ gels in the presence of lysozyme was significantly higher than that in the absence of lysozyme $(p<0.05)$. There was a significant difference $(p<0.05)$ between DCh/ $\beta$-GP/EA1 in the absence of lysozyme and $\mathrm{DCh} / \beta-\mathrm{GP} / \mathrm{EA} 0.1$ in the presence of lysozyme mainly due to initial loading difference. This result demonstrated that the release rate of ellagic acid from the $\mathrm{DCh} / \beta$-GP gels was affected by initial loading and chitosan lytic enzymatic activity. It is worth noting that the release profiles in both absence and presence of lysozyme showed a sustained slow release, rather than an initial burst effect.

\subsubsection{In vitro Enzymatic Degradation}

To determine the biodegradation rate of the $\mathrm{DCh} / \beta$-GP gels in vitro, the amounts of enzymatic-hydrolyzed DCh/ $\beta$-GP gels were measured by ninhydrin assay for chitosan vehicle alone and with 2 concentrations of EA described previously. Figure 5.8 shows the cumulative degradation rates of the $\mathrm{DCh} / \beta$-GP gels for 3 weeks incubation in lysozyme medium. The results showed significant increases in free amino groups corresponding to $\mathrm{N}$-glucosamine from all the groups after 3 weeks incubation compared with 1 day incubation $(p<0.05)$, suggesting a continuous degradation of the $\mathrm{DCh} / \beta-\mathrm{GP}$ gels. The $\mathrm{DCh} / \beta$-GP/EA0.1 exhibited significantly lower enzymatic-degradation rates than the other groups at 7,14 , and 21 day $(p<0.05)$. The higher initial loading of ellagic acid in the $\mathrm{DCh} / \beta$-GP/EA1 led to a significantly higher degradation rate after 2 weeks incubation compared with the DCh/ $\beta$-GP $(p<0.05)$.

\subsubsection{Cytotoxicity of $\beta-G P$ Solution}

To evaluate the effect of the $\beta$-GP concentration on cell viability, human HS68 newborn fibroblasts were cultured with media containing different concentrations of $\beta$ GP solution. As shown in Figure 5.9, cell activity measured by a MTS assay decreased at $210 \mathrm{mM} \beta-\mathrm{GP}$ or higher $(p<0.05$ for all time points). While there were significant increases in cell activity in culture media containing $0-131 \mathrm{mM}$ of $\beta$-GP solution during 3 days incubation $(p<0.05)$, indicating no cytotoxic effect on cell survival and growth. There was an inverse relationship between $\beta$-GP and cell activity that became significant $(p<0.05)$ at $88 \mathrm{mM}$ of $\beta$-GP and $131 \mathrm{mM}$ of $\beta$-GP after 3 days. This result suggests that 


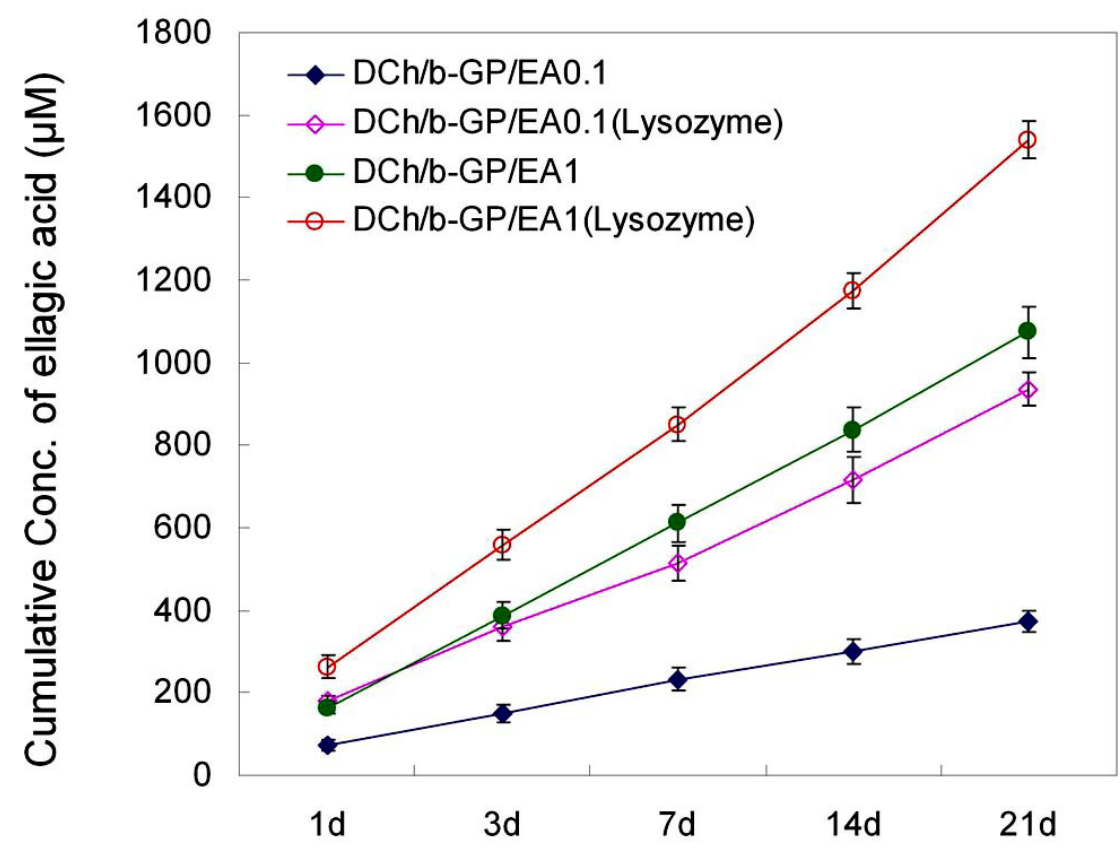

Figure 5.7 In vitro cumulative amount of the ellagic acid released from the DCh/ $\beta-\mathrm{GP} / \mathrm{EA}$ gels in the absence of lysozyme or in the presence of lysozyme. The cumulative amount of the released ellagic acid was determined as a function of time by a UV spectrophotometer at $260 \mathrm{~nm}$. Each value represents the mean $\pm \mathrm{SD}(\mathrm{n}=3$ per each group). 


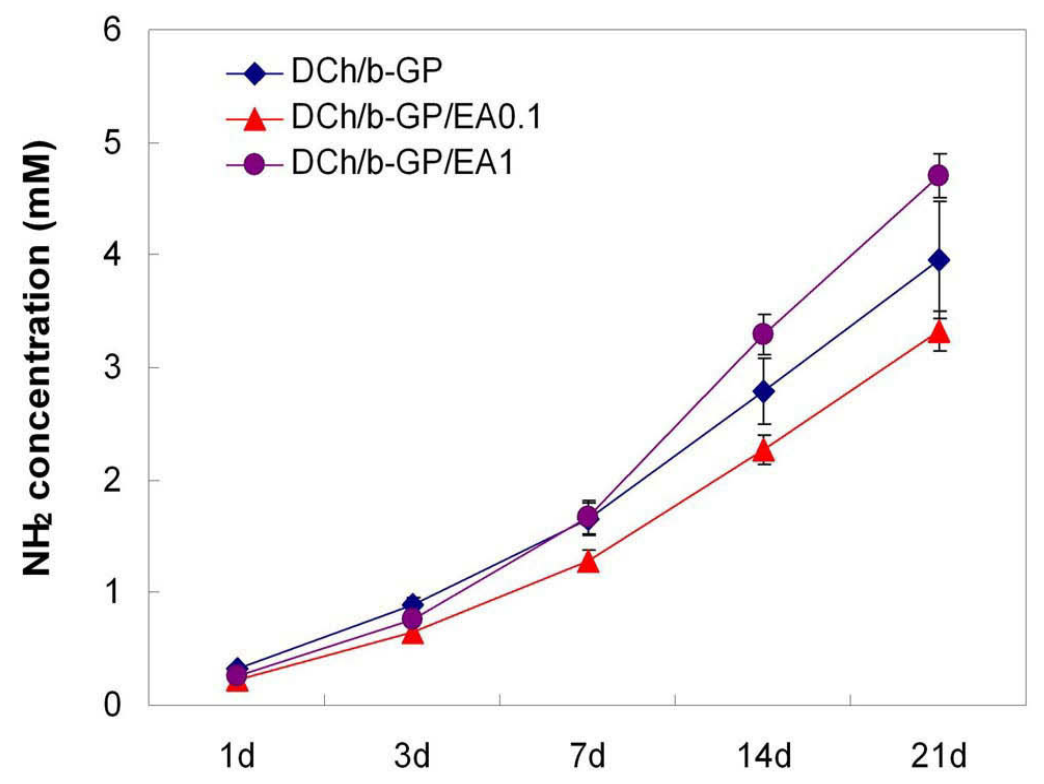

Figure 5.8 In vitro cumulative enzymatic degradation of the $\mathrm{DCh} / \beta$-GP gels. The amount of free amino groups corresponding to $\mathrm{N}$-glucosamine was determined as a function of time by a ninhydrin assay at $550 \mathrm{~nm}$ : Each value represents the mean $\pm \mathrm{SD}(\mathrm{n}=3$ per each group). 


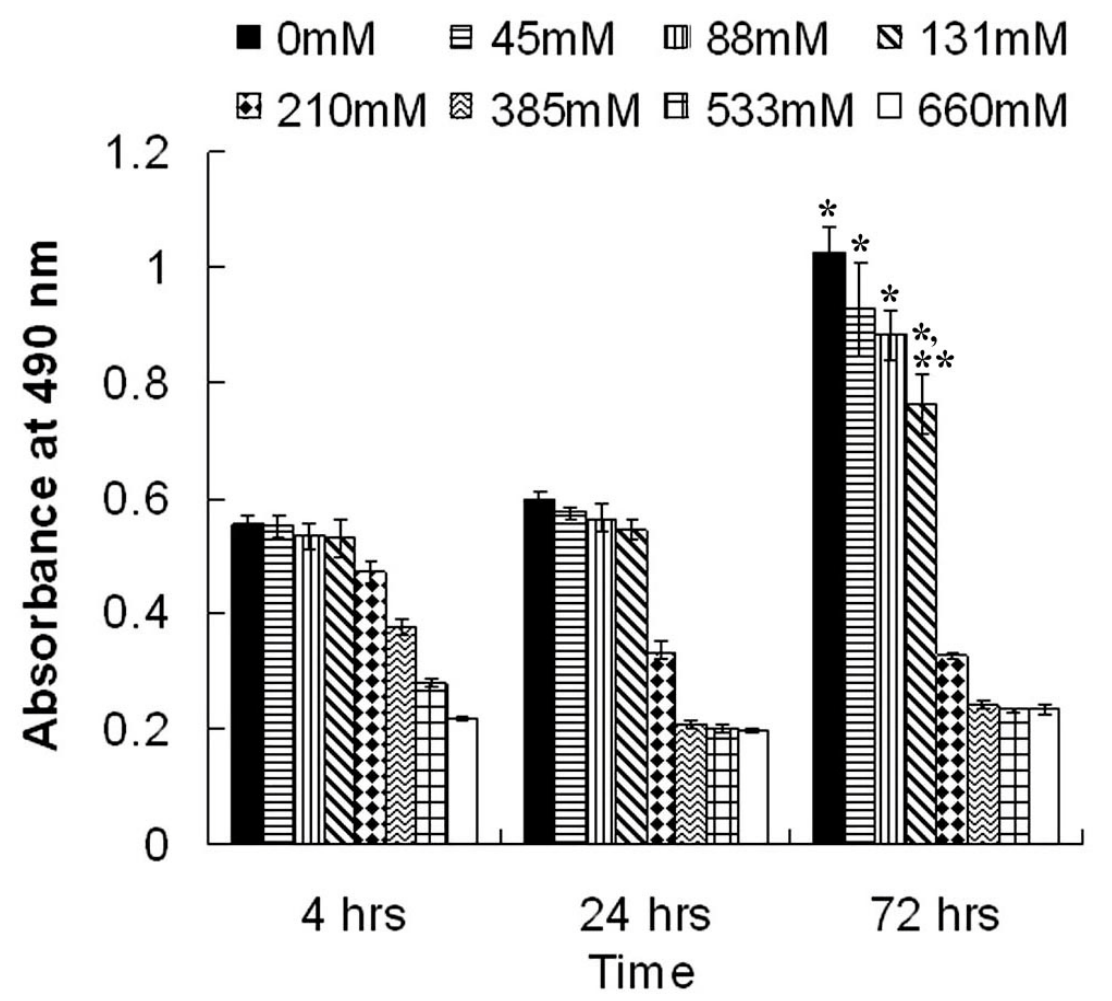

Figure 5.9 Viability of HS68 human newborn fibroblasts via direct culture by the MTS assay. The cells were cultured at a density of 30,000 cells per well without changing medium for 4, 24, and 72 hours. The absorbance is expressed as a measure of the cell viability into culture medium containing different concentrations of $\beta$-GP solution for 3 days incubation. ${ }^{*}$ denotes significant increase in cell viability compared with 4 and 24 hours incubation $(p<0.05)$. ** denotes significantly different cell viability compared with $0 \mathrm{mM}, 45 \mathrm{mM}$, and $88 \mathrm{mM}$ of $\beta$-GP at 3 days incubation $(p<0.05)$. Each value represents the mean $\pm \mathrm{SD}(\mathrm{n}=4$ per each group). 
cell viability was affected by $\beta$-GP solution in a dose-dependent manner.

\subsubsection{Cytotoxicity of Chitosan/ $\beta$-GP Gel}

To investigate the effect of the DCh/ $\beta$-GP gels on human HS68 newborn fibroblasts via culture media, cytotoxicity evaluation was performed by a MTS assay and the cell morphology was observed by microscope (Nikon ECLIPSE TE-2000-U). Figure 5.10 shows the photomicrographs of the calcein AM stained H68 fibroblasts. The result indicated that cells encapsulated inside gels were viable in a round shape (Figure 5.10a) while cells on the bottom of the well plate were spindle-like in shape and proliferated for 3 days incubation (Figure 5.10b). In addition, cells which were encapsulated inside gels for 3 days were transferred to a new 24-well plate and cultured for 3 days. They changed their morphology and proliferated on the surface of the cell culture plate (Figure 5.10c). This result suggests that cells encapsulated inside gels were viable in their round shape until they reached the rigid flat surface on which cells were able to move, grow, and proliferate.

Lower $\beta$-GP concentration vehicles were desirable for biocompatibility. Dialysis of chitosan solution (DCh) before addition of $\beta$-GP reduced the amount of $\beta$-GP needed to achieve a $\mathrm{pH}$ of 7.2, which was physiologically acceptable and suitable for the gelation compared to undialyzed chitosan solution $(\mathrm{Ch})$. The difference in biocompatibility is illustrated in Figure 5.11 which shows the cytotoxicity of the chitosan gels containing $0.088 \mathrm{M}$ of $\beta$-GP $(\mathrm{DCh} / \beta-\mathrm{GP}$; final $\mathrm{pH} 7.23)$ compared to $1.155 \mathrm{M}$ of $\beta-\mathrm{GP}(\mathrm{Ch} / \beta-\mathrm{GP}$; final $\mathrm{pH}$ 7.17). There were significant decreases in cell activity with $1.155 \mathrm{M}$ of $\beta$-GP after 4 hours compared with 24 hours and 3 days $(p<0.05)$, indicating that $1.155 \mathrm{M}$ of $\beta$ GP is toxic.

However, cells in the chitosan gel containing $0.088 \mathrm{M}$ of $\beta$-GP significantly increased cell activity after 3 days $(p<0.05)$. This result was consistent with the photomicrographs shown in Figure 5.10, indicating that DCh/ $\beta$-GP $(0.088 \mathrm{M}$ of $\beta$-GP) is not cytotoxic and allowed cell growth and proliferation. However, $\mathrm{Ch} / \beta-\mathrm{GP}(1.155 \mathrm{M}$ of $\beta$-GP) was toxic. Dialysis reduced the amount of $\beta$-GP needed to achieve $\mathrm{pH}$ 7.2. Dialysis was a key step to reduce the amount of $\beta$-GP needed to achieve a fluid, biocompatible vehicle that gels rapidly at body temperature.

\subsubsection{In vitro Anti-Tumor Activity of Chitosan/ß-GP Gel}

We investigated the anti-proliferative effect of the DCh/ $\beta$-GP gels loaded with ellagic acid on human U87 glioblastoma and rat C6 glioma cells by a MTS assay. As shown in Figure 5.12a, there were significant increases in viability of U87 glioblastoma cultured with the $\mathrm{Ch} / \beta$-GP gels after 72 hours incubation compared with 4 hours $(p<0.05)$. However, the viability of the U87 cells was significantly decreased when they were cultured with the $\mathrm{Ch} / \beta-\mathrm{GP} / \mathrm{EA} 0.1$ or $\mathrm{Ch} / \beta$-GP/EA1 for 72 hours incubation compared with 4 hours. There was a significant difference in the viability of the U87 cells between 


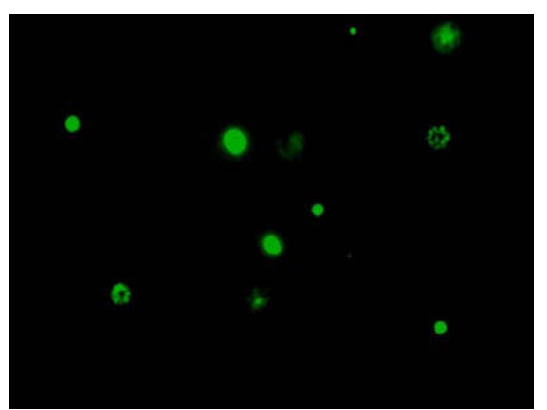

(a)

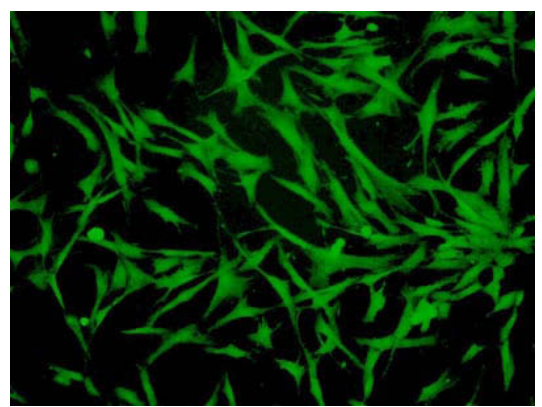

(b)

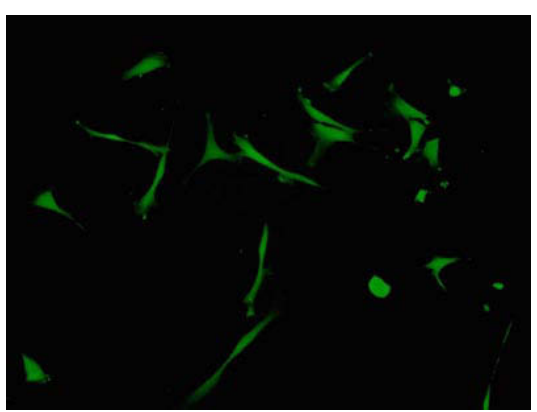

(c)

Figure 5.10 Photomicrographs showing viability of HS68 human newborn fibroblasts via the direct culture on the DCh/ $\beta$-GP gel. (a) cells encapsulated inside gel; (b) cells cultured outside gel; (c) cells in (a) were transferred to new 24-well plates and cultured for 3 days. The cells were cultured on the chitosan gels $(0.088 \mathrm{M}$ of $\beta$-GP) in 24 -well plates at a density of 60,000 cells per well without changing medium for 3 days. The final $\mathrm{pH}$ of the chitosan/ $\beta$-GP solution was 7.23. $(\mathrm{MAG}=\times 100)$. 


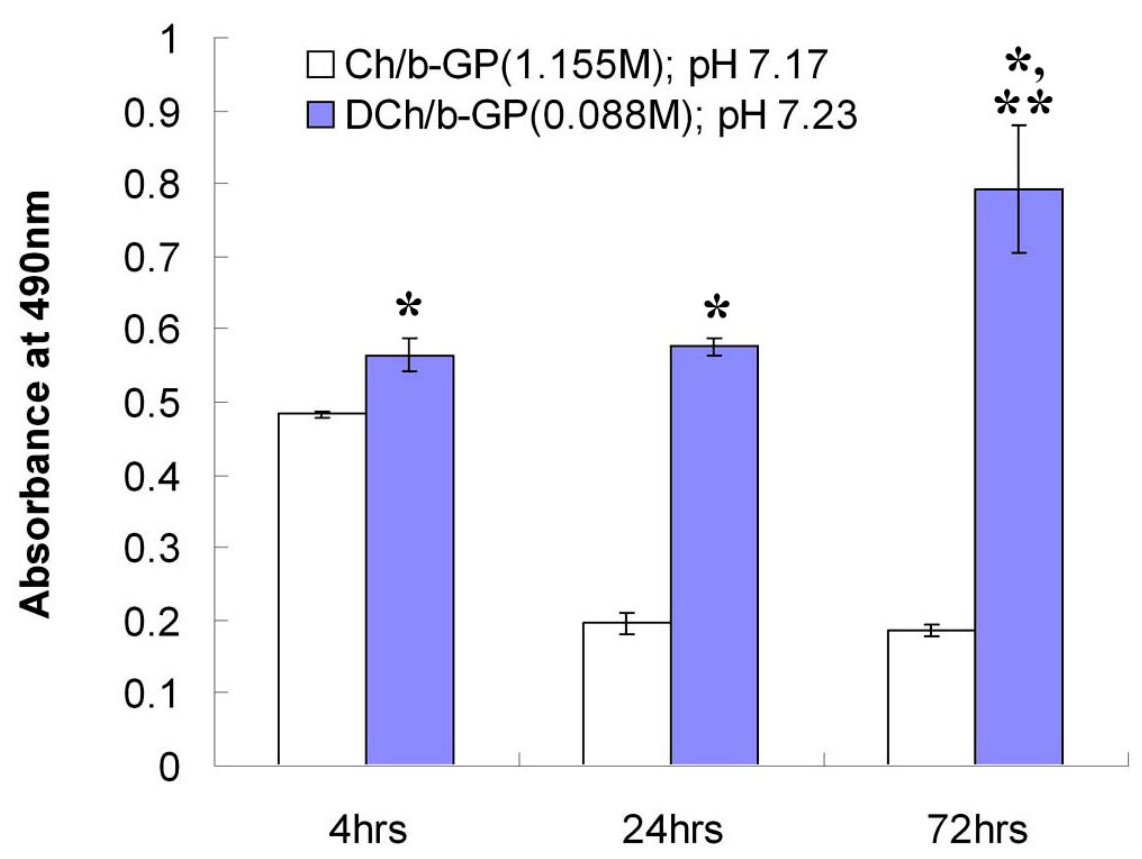

Figure 5.11 Viability of HS68 human newborn fibroblasts via indirect culture by the MTS assay. The cells cultured on the permeable PET membrane were affected by the chemicals released into the culture media from the chitosan gels. The chitosan $/ \beta$-GP gels were prepared with $0.088 \mathrm{M}$ of $\beta$-GP solution or $1.155 \mathrm{M}$ of $\beta$-GP solution. The final $\mathrm{pH}$ of each chitosan/ $\beta$-GP solution was 7.23 and 7.17 , respectively. The initial cell density was 60,000 cells per well and cells were cultured without changing medium for 4, 24, and 72 hours. The absorbance is expressed as a measure of the cell viability via cell culture media for 3 days incubation. $*$ denotes significantly different cell viability compared with the $\mathrm{Ch} / \beta$-GP $(1.155 \mathrm{M})$ for 3 days incubation $(p<0.05) . * *$ denotes significantly different cell viability compared with 4 and 24 hours incubation $(p<0.05)$. Each value represents the mean $\pm \mathrm{SD}$ ( $\mathrm{n}=3$ per each group). 


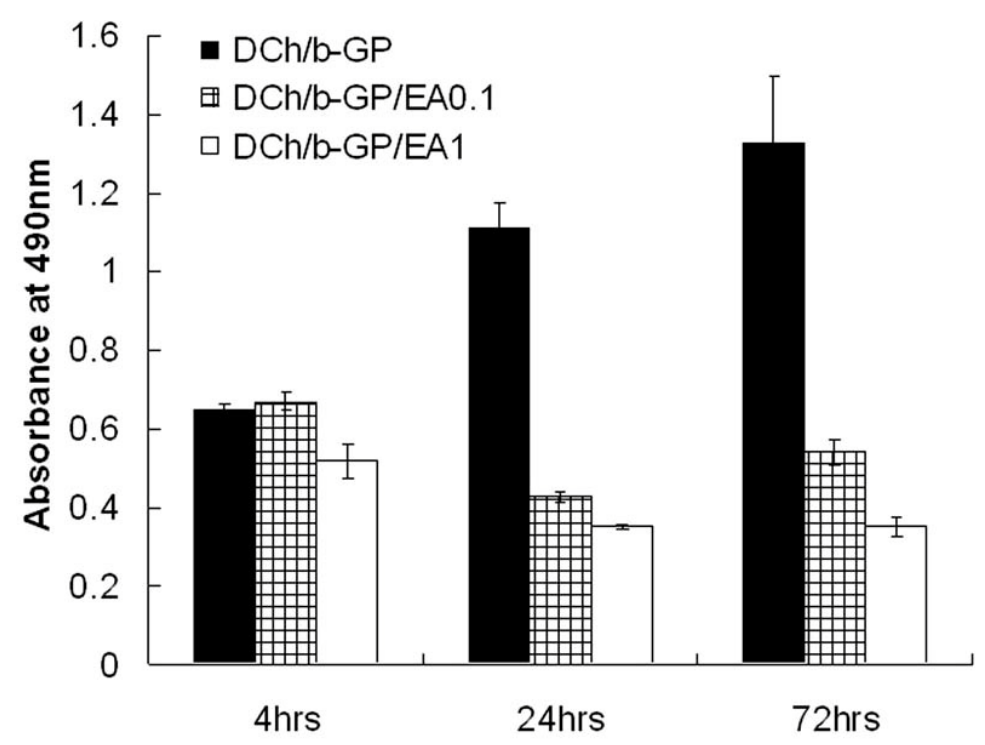

(a)

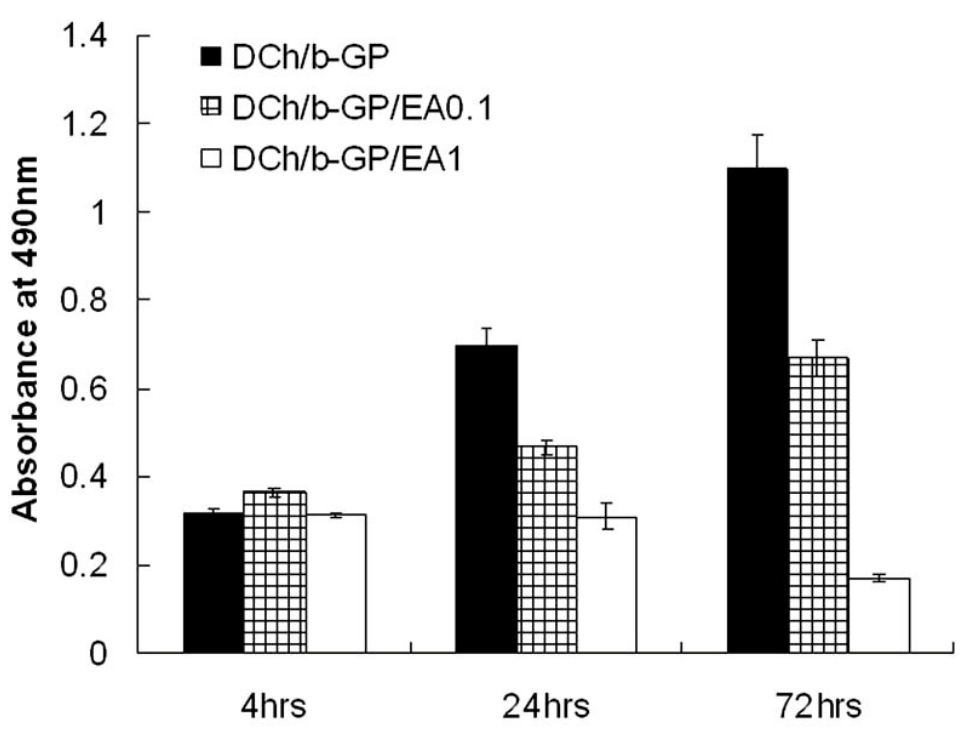

(b)

Figure 5.12 Viability of cancer cells via the indirect culture by the MTS assay. (a) human U87 glioblastoma; (b) rat C6 glioma. The cancer cells seeded onto the permeable PET membrane were affected by the ellagic acid released into the culture medium. The absorbance is an indicator of the viability of the cells for 4, 24, 72 hours incubation. Each value represents the mean $\pm \mathrm{SD}(\mathrm{n}=3$ per each group). 
the $\mathrm{Ch} / \beta$-GP/EA0.1 and the $\mathrm{Ch} / \beta$-GP/EA1 for 3 days incubation $(p<0.05)$, indicating the anti-tumor effect of the $\mathrm{Ch} / \beta$-GP gel in an ellagic acid concentration dependent manner.

In addition, as shown in Figure 5.12b, a significant increase in the viability of C6 glioma cells was observed when they were cultured with the $\mathrm{Ch} / \beta$-GP gel or the $\mathrm{Ch} / \beta$ GP/EA0.1 for 72 hours compared with 4 and 24 hours incubation $(p<0.05)$. This result suggests that there was no inhibitory effect of the $\mathrm{Ch} / \beta$-GP/EA0.1 on the survival and growth of cells. However, there was a significant difference between the $\mathrm{Ch} / \beta$-GP and the $\mathrm{Ch} / \beta$-GP/EA0.1 after 24 hours incubation $(p<0.05)$, indicating the effect of ellagic acid on cell growth. There was a significant decrease in the viability of the $\mathrm{C} 6$ glioma cells when cultured with the $\mathrm{Ch} / \beta$-GP/EAl after 72 hours compared with 4 and 24 hours incubation $(p<0.05)$. This result demonstrated that the $\mathrm{Ch} / \beta$-GP gel loaded with ellagic acid could inhibit the growth of cancer cells in an ellagic acid concentration dependent manner.

\subsection{Discussion}

Recently, thermo-sensitive injectable systems have been studied to carry therapeutic agents, cells or molecules for local delivery [Hoemann 2005, Molinaro 2002, Ta 2008]. In particular, chitosan based injectable gels have notable potential as a drug carrier regarding controlled release and loading capacity [Bae 2006, Roughley 2006, Ruel-Gariepy 2000, 2004, Ta 2009, Tien 2004]. This study focused development and characterization of a thermo-sensitive $\mathrm{Ch} / \beta$-GP gel regarding thermal gelation and biocompatibility. The anti-tumor effect of the $\mathrm{Ch} / \beta$-GP gel on brain cancer cells were also evaluated according to different concentrations of ellagic acid. It was hypothesized that a heat-induced chitosan gel delivers anti-cancer activity of ellagic acid via a simple injection.

The gelation of a chitosan/ $\beta$-GP solution is thermally induced via several types of interactions such as electrostatic attractions, hydrogen bonding, electrostatic repulsion, and hydrophobic effect [Chenite 2001, Cho 2005, Hoemann 2007, Wu 2006]. Chitosan polymer contains hydrogen bonding favoring groups such as hydroxyl $(-\mathrm{OH})$, amine ($\mathrm{NH}_{2}$ ), amide $(-\mathrm{NH}-\mathrm{C})$ and carbonyl $(-\mathrm{C}=\mathrm{O})$ groups. It is positively charged in an acidic solution due to the protonation of the free amine groups, which causes electrostatic repulsion between chitosan molecules. When a weak base of disodium $\beta$-GP solution is added into an acidic chitosan solution, it increases $\mathrm{pH}$ and also induces electrostatic attractions between positively charged chitosan $\left(\mathrm{NH}_{3}{ }^{+}\right)$and negatively charged phosphate molecules $\left(-\mathrm{HPO}_{4}{ }^{-}\right.$or $\left.-\mathrm{PO}_{4}{ }^{2-}\right)$ of $\beta$-GP. It also reduces electrostatic repulsion between the chitosan chains due to charge neutralization by $\beta$-GP anion, which results in an increase in hydrogen bonding interactions along chitosan interchains [Cho 2005]. Generally, chitosan molecules in an acidic solution immediately form a hydrated precipitate by adding a strong base, such as $\mathrm{NaOH}$, due to the reduced charge density along chitosan chains, which subsequently induces extensive hydrogen bonding and hydrophobic interactions between chains. This results in an inability to maintain chitosan molecules in a solution at physiologically acceptable pH [Berger 2004, Chenite 2001, Nair 2007]. 
However, the glycerol molecules of $\beta$-GP form a shield of water around the chitosan molecules, maintain chitosan chains in their native form, and inhibit their aggregation at low temperature. Therefore, polyols and sugars have been used as stabilizer for protein or biopolymer to prevent structural changes [Back 1979, Chenite 2000, Jarry 2002]. With increasing temperature, chitosan-chitosan interactions become dominant resulting in a phase transition from a liquid to a gel while hydrogen bonding interactions are reduced. After breaking of hydrogen bonding, phosphate groups function as a proton sink after chitosan chains release protons [Ta 2008]. The chitosan chains subsequently are brought close to precipitation resulting in the gelation of the chitosan/ $\beta$ GP formulation. Several studies have reported that phosphate groups of $\beta$-GP do not induce ionic crosslinking with the chitosan molecules [Chenite 2001, Cho 2005, Ta 2008]. Instead, the hydrophobic effect has been considered the main force to induce thermal gelation of the chitosan/ $\beta$-GP system.

In the present study, the FTIR spectra revealed that the protonation of phosphate groups in the $\mathrm{Ch} / \beta$-GP solution occurred with increasing temperature. This result indicates the transfer of protons $\left(\mathrm{H}^{+}\right)$in the $\mathrm{Ch} / \beta$-GP solution during thermal gelation. As shown in Figure 5.2, the diprotonated phosphate $\left(-\mathrm{H}_{2} \mathrm{PO}_{4}\right)$ groups appeared in the $\mathrm{Ch} / \beta$ $\mathrm{GP}$ at $37^{\circ} \mathrm{C}$, suggesting the nonprotonated phosphate $\left(-\mathrm{PO}_{4}{ }^{2-}\right)$ ions accepted protons $\left(\mathrm{H}^{+}\right)$ with increasing temperature [Arai 2001, Elzinga 2007]. As a result, there is a decrease in the protonation around the chitosan molecules, indicating decreased solubility of chitosan molecules. At low temperature, water molecules surround the chitosan chains. Heat increases the entropy of the chitosan solution, inducing chitosan to release protons. Consequently, the removal of hydrating water around chitosan molecules increases interactions between chitosan molecules. If there are sufficient phosphate groups accepting protons, sol-gel transition occurs [Chenite 2000, Cho 2005, Ta 2008]. This explains why dialyzed chitosan solution (DCh) requires lower concentrations of $\beta-\mathrm{GP}$ than the undialyzed chitosan solution for the sol-gel transition.

In general, the gelation of the injectable chitosan gel can be controlled by the degree of deacetylation of chitosan, concentration of chitosan, the amount of polyol salt, temperature, and the final $\mathrm{pH}$ of the gel forming solution [Ta 2008]. In the present study, the gelation of the chitosan formulation was more dependent on the final $\mathrm{pH}$ rather than the concentration of the $\beta$-GP solution. The chitosan solutions containing both high concentration $(1.155 \mathrm{M})$ of $\beta$-GP and low concentration $(0.088 \mathrm{M})$ of $\beta$-GP could induce thermal gelation around body temperature because their final $\mathrm{pH}$ values were around 7.2. Gelation of the chitosan/ $\beta$-GP system was affected by a combination of the final $\mathrm{pH}$, gelation time, and temperature. In addition, the rheological analysis also demonstrated that hydrophobic interactions played the most important role in the gelation of the chitosan/ $\beta$-GP solution [Chenite 2000, Cho 2005, 2006]. Generally, the strength of the gel is determined by the magnitude of the storage modulus $\left(G^{\prime}\right)$ or the great difference between the storage modulus $\left(G^{\prime}\right)$ and the loss modulus $\left(G^{\prime \prime}\right)$. The result showed that the chitosan solution containing a higher concentration $(1.155 \mathrm{M})$ of $\beta$-GP showed a lower storage modulus $\left(G^{\prime}\right)$, probably due to lower hydrophobic interaction between chitosan chains. This result indicated that the ionic interaction between ammonium groups of 
chitosan and phosphate groups of $\beta$-GP did not significantly affect the thermal gelling mechanism [Cho 2005].

The release of ellagic acid from the chitosan/ $\beta$-GP gels is thought to take place by diffusion and could be accelerated by enzymatic degradation. This study showed that the loaded ellagic acid also affected the organization of the chitosan/ $\beta$-GP gel network resulting in slower degradation rate of DCh/ $\beta$-GP/EA0.1 than that of chitosan/ $\beta$-GP gel. This result suggested that ellagic acid might be involved in the formation of chitosan/ $\beta$ GP gels and bind to chitosan molecules via ionic crossliking. However, chitosan $/ \beta-G P$ gels loaded with higher concentrations of ellagic acid showed faster degradation rates. This result was different from the previous study about $\mathrm{Ch} / \mathrm{EA}$ composite films. As discussed in chapter 4 [Kim 2009], the in vitro enzymatic degradation behavior of the $\mathrm{Ch} / \mathrm{EA}$ composite films was ellagic acid concentration dependent. The composite films with a higher weight percentage of ellagic acid exhibited slower degradation rates. This might be because ellagic acid was conjugated with the chitosan polymer via covalent bonds or ionic forces and embedded into the chitosan polymer network. However, this study showed that the addition of the $\beta$-GP solution changed the degradation rate due to a different mechanism of polymerization. This result may be because the main driving force for gelation is the hydrophobic effect rather than ionic crosslinking [Cho 2006]. The ellagic acid in the $\mathrm{Ch} / \beta-\mathrm{GP} / \mathrm{EA} 1$ was mostly entrapped along chitosan molecules physically and released fast when the chitosan/ $\beta$-GP gel was swollen under acidic conditions. As a result, the $\mathrm{Ch} / \beta$-GP gel degrades faster than the $\mathrm{Ch} / \mathrm{EA}$ composite film along the pores of the $\mathrm{Ch} / \beta$-GP gel and could be accelerated by an enzymatic activity. In addition, the morphological observation of the lypholized gels revealed different pore structures in the gel in the presence and absence of ellagic acid. The difference in pore texture is due to incorporation of ellagic acid into the $\mathrm{Ch} / \beta-\mathrm{GP}$ gel. This porous structure of the $\mathrm{Ch} / \beta$-GP gel is obviously different from the surface morphology of the composite film. Structural conformation of a material plays a significant role in the release of drugs [Ta 2009]. Therefore, the diffusion rate of gel materials is greater than that of film. These results demonstrated that ellagic acid is released by a combination of diffusion through pores as well as polymer matrix degradation.

The findings of this study also demonstrated that the concentration of $\beta$-GP significantly influenced the cell viability. To begin the sol-gel transition thermally, it is necessary to neutralize an acidic chitosan solution by adding a certain amount of $\beta$-GP up to the physiologically acceptable $\mathrm{pH}$ range. As shown in the cell viability results, however, high concentration of $\beta$-GP significantly reduced cell viability, indicating the toxic effect of the $\beta$-GP solution [Hoemann 2007]. 1.155 $\mathrm{M}$ of $\beta$-GP solution was required to increase the $\mathrm{pH}$ of an acidic chitosan solution up to a $\mathrm{pH}$ of 7.2, at which the chitosan solution underwent thermal gelation at $37^{\circ} \mathrm{C}$. In the present study, a dialysis membrane was used to increase the $\mathrm{pH}$ of an acidic chitosan solution before adding the $\beta$ GP solution. Accordingly, neutralization of the acidic chitosan solution could reduce the concentration of $\beta$-GP to reach $\mathrm{pH} 7.2$, resulting in improved cell viability as well as thermal gelation at $37^{\circ} \mathrm{C}$.

Cell adhesion, growth, and proliferation on the material surface are sequential 
responses which are essential for cell survival and function [Dubin-Thaler 2004, Aplin 2002]. Cells are influenced by several factors in their environment. These contain chemical and physical interactions with neighboring cells and the extracellular substrate as well as chemical factors in the serum. As shown in Figure 5.9, the morphological changes of cells reflected their cellular response to the environmental materials. The cells on the bottom of well plates changed their morphology from a circular shape to spindlelike features over time, while cells encapsulated inside the gel remained in their initial round shape but were still viable. This result suggests that the chitosan/ $\beta$-GP gel was biocompatible and allowed cells to remain viable until they reached the rigid surface, allowing them to move, grow, and proliferate.

As shown in the study of the anti-tumor activity, the chitosan/ $\beta$-GP gel loaded with ellagic acid could inhibit the growth of cancer cells in an ellagic acid concentration dependent manner. This result was consistent with previous studies, showing the antiproliferative effect of the $\mathrm{Ch} / \mathrm{EA}$ composite films against skin and brain cancer cells [Kim 2009]. The Ch/EA composite films could induce the accumulation of the tumor suppressor protein p53 and increase caspase-3 activation, which preceded an induction of apoptosis. The findings from studies of the $\mathrm{Ch} / \mathrm{EA}$ composite films showed the anti-tumor activity of ellagic acid via a local delivery system with a surgical procedure for implantation. In the present study, ellagic acid was delivered via an injection of a liquid formulation. The results of this study demonstrated that a heat-induced chitosan gel could deliver the anti-cancer activity of ellagic acid and be considered as a promising delivery method for a local chemotherapy.

\subsection{Conclusion}

This study was designed to develop a chitosan/ $\beta$-glycerophosphate $(\beta$-GP) gel to deliver ellagic acid via a simple injection and effectively prevent local recurrence of cancer cells. The chitosan/ $\beta$-glycerophosphate ( $\beta$-GP) solution formed heat-induced gel network at body temperature, and the gelation temperature and time were affected by the final $\mathrm{pH}$ of the chitosan/ $\beta$-GP solution. The ellagic acid was released from the chitosan $/ \beta$ GP gels by pores of gel network as well as polymer matrix degradation. A dialyzed chitosan solution could improve biocompatibility, indicated by 4 times higher cell viability than undialyzed chitosan gel at 3 days culture. Viability of U87 cells and C6 cells cultured on chitosan gels containing $1 \%(\mathrm{w} / \mathrm{v})$ of ellagic acid were lower than the same cells on chitosan gels at 3 days incubation by 3.8 times and 6.5 times, respectively. Therefore, the chitosan $/ \beta$-GP gels loaded with ellagic acid could inhibit the cancer cell growth in an ellagic acid concentration-dependent manner. This study has demonstrated that the chitosan/ $\beta$-GP delivery system is a promising biomaterial for cancer treatment as a local chemotherapy. 


\section{CHAPTER 6. DISCUSSION}

\subsection{Overall Goals}

The objective of this project was the utilization of ellagic acid using an implantable chitosan polymer for a sustained local delivery. It was hypothesized that chitosan/ellagic acid composite materials have anti-cancer activity at targeted sites by inducing apoptotic death and inhibiting angiogenesis. This chapter summarizes the specific findings of each study with regard to hypotheses in Chapter 2. Section 6.2 discusses development and characterization of chitosan/ellagic acid composite films in terms of chemical structure, crystallinity, surface morphology, degradation behavior, and release profile. Section 6.3 discusses in vitro anti-cancer activity of chitosan / ellagic acid composite films. This section emphasizes anti-cancer activity of the composite films via direct or indirect culture and potential anti-cancer mechanisms. Section 6.4 discusses in vivo anti-cancer activity of composite films using a glioma brain cancer model. Anticancer activity of the composite film is evaluated and compared with control groups. Section 6.5 discusses a thermo-sensitive injectable gel for the delivery of ellagic acid. This section emphasizes thermal gelation of chitosan polymer and biocompatibility of the delivery system. Finally, section 6.6 provides conclusions of this project.

\subsection{Development of the Chitosan/Ellagic Acid Composite Films}

Chitosan/ellagic acid composite films were fabricated to utilize the anti-cancer properties of ellagic acid at local targeted sites. It was hypothesized that ellagic acid is chemically attached and embedded into chitosan polymer, expressing a new form of ellagic acid. In order to test this hypothesis, chitosan/ellagic acid composite films were characterized using FTIR, SEM, water contact angle, and XRD. Their degradation behavior and release profiles were investigated. These investigations provided the information regarding surface chemistry and morphology of the composite films. Studies of surface chemistry indicated that there exist chemical reactions between chitosan and ellagic acid, including amide and ester linkages. This is because the carboxylic groups of the ellagic acid combined with the hydroxyl and amine groups of the chitosan [Tien 2003, Wang 2004]. The surface roughness and wettability of the composite films were significantly affected by concentration of ellagic acid. Composite films became rougher and more hydrophilic compared with the chitosan film. In addition, crystal structure of ellagic acid was observed with increasing concentration of ellagic acid. These findings suggested that ellagic acid was chemically bound and physically entrapped into the chitosan network, and its functional groups such as hydroxyl and carboxyl groups influenced surface chemistry [Amaral 2006, Neamnark 2007]. These results support hypothesis 1 .

A major target in local drug delivery is to overcome an initial burst effect, leading to a rapid loss of drug efficacy and distributing toxicity non-specifically into body [Dash 1998, Park 1996]. Drug release from polymeric delivery system is governed by 
polymer composition, polymer characteristics, polymer degradation, drug loading, drug solubility, and drug/polymer interactions [Gunatillake 2003]. In vitro release profiles of the ellagic acid clearly demonstrated a sustained slow release of ellagic acid without an initial burst release. The chitosan/ellagic acid composite films were biodegradable by enzymatic hydrolysis. In this regard, the presence of lysozyme accelerated the release profile of ellagic acid compared with the absence of lysozyme. Some characteristics of chitosan and ellagic acid such as solubility, DDA of chitosan, and chitosan/ellagic acid interactions contributed to the sustained slow release of ellagic acid from composite films. The degradation rate of chitosan films can be slowed down by using a chitosan polymer with high degree of deacetylation (DDA) and molecular weight [Li 2007, Wang 2006]. In addition, ellagic acid is slightly soluble in medium at $\mathrm{pH}$ 6.0. These results support hypothesis 2 . The findings from these studies suggest that the release profile of ellagic acid is governed by chitosan characteristics, degradation, chitosan/ellagic acid interactions, ellagic acid loading, and solubility.

\subsection{In vitro Anti-Cancer Activity of Composite Films}

It was hypothesized that the chitosan/ellagic acid composite films inhibit growth of cancer cells in an ellagic acid concentration dependent manner. In order to test this hypothesis, viable cancer cells and their activities were monitored on the composite films. Studies of anti-cancer activity indicated that composite films lead to cancer cell death in an ellagic acid concentration-dependent manner. The morphological changes of cancer cells cultured on composite films reflected a direct cellular response to the composite films. Cell adhesion, growth, and proliferation correspond with cell survival and function [Findlay 2002, Willingham 1999]. Cancer cells cultured on chitosan films containing 0, 0.05 , and $0.1 \%(\mathrm{w} / \mathrm{v})$ of ellagic acid initially aggregated, indicating initial inhibitory effect of composite films. However, after 3 days incubation, cancer cells survived and spread, suggesting these composite films could not induce anti-cancer activity. Initial inhibitory effect of the composite films is probably due to the combination effect of chitosan and ellagic acid. Some evidences suggested that chitosan induces anti-bacterial and anti-tumor properties attributed to the tumor necrosis factor- $\alpha$ (TNF- $\alpha$ ) in human monocytes [Muzzarelli 1997, Ravi Kumar 2006]. In contrast, cancer cells cultured on chitosan films containing 0.5 and $1 \%(\mathrm{w} / \mathrm{v})$ of ellagic acid remained in the initial round shape and could not grow and proliferate, indicating anti-cancer activity of composite films in an ellagic acid concentration dependent manner, thus supporting hypothesis 3 .

In order to test hypothesis 4 stating that chitosan/ellagic acid composite films localize therapeutic effect via slow release of ellagic acid, anti-cancer activity of composite films was monitored via indirect cell culture. This study demonstrated cancer cells in the absence of direct contact with the composite film significantly proliferated, indicating the slow release of the ellagic acid into the cell culture media. This result is consistent with the release study in section 6.2. This localized effect was also monitored by observing cell morphological changes at the interface of the composite films in chapter 3 [Kim 2009]. However, there exists significant difference between a chitosan film and a chitosan film containing $1 \%(\mathrm{w} / \mathrm{v})$ of ellagic acid, indicating the effect of 
released ellagic acid into culture medium. These findings support hypothesis 4 .

The anti-cancer mechanism of the chitosan/ellagic acid composite films was investigated in chapter 3 and 4 . It was hypothesized that the composite films have anticancer activity by inducing apoptosis of cancer cells and suppressing angiogenesis [Khan 2007, Labrecque 2005]. The results demonstrated that the chitosan/ellagic composite films induce apoptotic cell death via accumulation of the tumor suppressor protein p53 and increase in caspase- 3 activation. These investigations suggest induction of apoptosis by the composite films would minimize inflammatory effects causing the damage in the surrounding tissue. In addition to the apoptosis mechanism, the tube formation of endothelial cells was monitored to investigate the ability of the composite films to inhibit angiogenesis. The results showed that the composite film (Ch/EA1) inhibits morphological differentiation of the HUVEC, indicating the effect of the composite film on suppressing angiogenesis. These findings support hypothesis 5.

\subsection{In vivo Anti-Cancer Activity of Composite Films in a Glioma Model}

It was hypothesized that a composite film delivers anti-cancer activity of ellagic acid effectively in a local glioma tumor model. Studies of the glioma animal model were designed according to preliminary data and literature reviews. Some evidences suggested that the significance of the effect depends on time at which treatment was initiated [Au 2001, Bello 2002, Giussani 2003]. In this study herein, the treatment group (Ch/EA20) was implanted subcutaneously on the fifth day after tumor inoculation. At this point, the tumors were small and visually detectable. A preliminary animal study, in which the treatment group (Ch/EA10) was implanted on the eleventh day after tumor inoculation, demonstrated there was no significant difference between a control group and a treatment group. This result suggests the significant effect corresponds with initial treatment time and ellagic acid loading. The findings of this animal study verified that the treatment group (Ch/EA20) significantly delayed tumor growth compared with an untreated group and a chitosan control group. In addition, severe weight loss of animals was not observed in all the groups. The histological analysis also confirmed the efficacy and safety of the $\mathrm{Ch} / \mathrm{EA}$ composite film at the local area. This result can be attributed to the slow and localized release of ellagic acid as discussed in section 6.3. These findings support hypothesis 6.

\subsection{Injectable Chitosan Gel Delivery System}

A chitosan based injectable hydrogel was investigated in terms of thermal gelation, biocompatibility, and anti-cancer activity. This study was designed to deliver ellagic acid via an injection of a liquid formulation. It was hypothesized that a liquid chitosan formulation forms a heat-induced chitosan gel at body temperature and delivers the anti-cancer activity of ellagic acid. A $\beta$-glycerophosphate $(\beta-\mathrm{GP})$ was employed in this study for induction of thermal gelation of chitosan solution. Several interactions during the gelation process are involved, including the loss of electrostatic repulsion, 
hydrogen bonding, and increased hydrophobic interaction. It was postulated that main driving force for gelation is the hydrophobic effect rather than ionic crosslinking [Cho 2006]. The findings of this study suggest that thermal gelation is governed by the $\mathrm{pH}$ of the chitosan/ $\beta$-GP solution and occurs at body temperature supporting hypothesis 7 . Additionally, this injectable gel is biocompatible and biodegradable. The release profile of ellagic acid from the chitosan gel represents sustained slow release and can be accelerated by an enzymatic activity. The chitosan/ $\beta$-GP gels loaded with ellagic acid inhibited cancer cell growth in vitro in an ellagic acid concentration-dependent manner. These results support hypothesis 8 . These findings of this study suggest that injectable chitosan/ $\beta$-GP gel is a promising delivery method as a local chemotherapy.

\subsection{Conclusion}

This project proposes an implantable polymeric delivery system for a sustained drug release and localized treatment. Chitosan/ellagic acid composite materials have been studied with respect to a local anti-cancer activity of ellagic acid in vitro and in vivo.

Composite films were fabricated for the sustained and slow release of ellagic acid via utilization of chitosan characteristics, chitosan/ellagic acid interactions, and ellagic acid concentration. From studies of composite films, it was observed that ellagic acid was incorporated into chitosan polymer and its anti-cancer activity could be localized in a dose-dependent manner. Localized effect of ellagic acid corresponds with slow release of ellagic acid into surrounding environments, inducing less toxic effect. It was postulated that anti-cancer mechanism of ellagic acid includes apoptosis and antiangiogenesis. These studies demonstrated that the chitosan/ellagic acid composite films induce anti-cancer activity by inducing apoptotic death and inhibiting angiogenesis in an ellagic acid concentration dependent manner.

To further improve the delivery method, studies of a chitosan base injectable gel were performed using $\beta$-glycerophosphate salt ( $\beta$-GP) for a local delivery of ellagic acid. The results demonstrated that the thermal gelation occurs at body temperature via hydrophobic interactions between chitosan molecules. Sol-gel transition was dependent on the $\mathrm{pH}$ of the gel formulation and temperature. The chitosan/ $\beta$-GP gel delivery system was biocompatible and could induce anti-cancer activity via a sustained release of ellagic acid. In conclusion, the chitosan/ellagic acid composite material is an implantable delivery system and provides sustained local therapy for cancer treatment. 


\section{CHAPTER 7. FUTURE DIRECTIONS}

The chitosan/ellagic acid composite materials herein showed as a promising local delivery for cancer treatment. However, most studies have been performed on a short term basis and therefore future experimental designs should be undertaken to determine the long term effects of these materials with regard to release profile, degradation behavior, and biological properties. The composite films degraded slowly with increasing concentration of ellagic acid. This may be desirable for a sustained release, but it may cause a long term complication in the normal tissue if it does not degrade completely after a therapeutic span. Generally, biodegradation of the polymeric delivery systems depends on polymer structure, composition, morphology, surface structure, molecular weight, shape, size, and etc [Dash 1998, Jain 2000]. The degradation of the composite materials, in this research, was governed by characteristics of chitosan, concentration of ellagic acid, chitosan/ellagic acid interactions, and fabrication methods (film or gel). Those factors are controllable and their changes may significantly affect the release rate and the biological properties of the composite materials. Therefore, further studies are required to investigate the optimal conditions according to the applications.

In view of the anti-cancer activity, the phenolic compounds including ellagic acid may be considered promising pharmaceutical agents in the future because of their druglike effects on human body. But, they are poorly absorbed into the body due to the limited bioavailability and high metabolism in human [Rahman 2006, Scalbert 2000]. From this research, the incorporation of the ellagic acid into the chitosan polymer could extend the use of biological properties of ellagic acid by delivering a specific local site. Therefore, it is necessary to establish the therapeutic strategies for the delivery of the phenolic compounds against human diseases including cancer. In fact, studies on identifying the mechanism of the action for the phenolic compounds in the local delivery may lead to new therapeutic targets. 


\section{LIST OF REFERENCES}

Aggarwal BB, Shishodia S. Molecular targets of dietary agents for prevention and therapy of cancer. Biochemical Pharmacology 2006;71:1397-1421.

Ahmad N, Adhami VM, Afaq F, Feyes DK, Mukhtar H. Resveratrol causes WAF-1/p21mediated G1-phase arrest of cell cycle and induction of apoptosis in human epidermoid carcinoma A431 cells. Clinical Cancer Research 2001;7:1466-1473.

Ahn D, Putt D, Kresty L, Stoner GD, Fromm D, Hollenberg PF. The effects of dietary ellagic acid on rat hepatic and esophageal mucosal cytochromes P450 and phase II enzymes. Carcinogenesis 1996;17(4):821-828.

Amaral IF, Granja PL, Melo LV, Saramago B, Barbosa MA. Functionalization of chitosan membranes through phosphorylation: Atomic force microscopy, wettability, and cytotoxicity studies. Journal of Applied Polymer Science 2006;102:276-284.

American Cancer Society. Cancer Facts and Figures, 2008. Atlanta, GA. American Cancer Society;2008.

Aplin AE, Hogan BP, Tomeu J, Juliano RL. Cell adhesion differentially regulates the nucleocytoplasmic distribution of active MAP kinases. Journal of Cell Science 2002;115:2781-2790.

Arai Y, Sparks DL. ATR-FTIR spectroscopic investigation on phosphate adsorption mechanisms at the ferrihydrite-water interface. Journal of Colloid and Interface Science $2001 ; 241: 317-326$.

Ashkenazi A, Dixit VM. Death receptors: signaling and modulation. Science 1998; 281(5381):1305-8.

Au JS, Jang SH, Zheng J, Chen CT, Song S, Hu L et al. Determinants of drug delivery and transport to solid tumors. Journal of controlled release 2001;74:31-46.

Avgeropoulos NG, Batchelor TT. New Treatment Strategies for Malignant Gliomas. The Oncologist 1999;4:209-224.

Back JF, Oakenfull D, Smith MB. Increased thermal stability of proteins in the presence of sugars and polyols. Biochemistry 1979;18(23):5191-5196.

Bae JW, Go DH, Park KD. Thermosensitive chitosan as an injectable carrier for local drug delivery. Macromolecular Research 2006;14(4):461-465.

Bai L, Zhu W. p53: Structure, function and therapeutic applications. Journal of Cancer Molecules 2006;2(4):141-153. 
Barch DH, Rundhaugen LM, Stoner GD, Pillay NS, Rosche WA. Structure-function relationships of the dietary anticarcinogen ellagic acid. Carcinogenesis 1996;17(2):265269.

Bello L, Giussani C, Carrabba G, Pluderi M, Lucini V, Pannacci M et al. Suppression of Malignant Glioma Recurrence in a Newly Developed Animal Model by Endogenous Inhibitors. Clinical Cancer Research. 2002;8:3539-3548.

Berger J, Reist M, Mayer JM, Felt O, Gurny R. Structure and interactions in chitosan hydrogels formed by complexation or aggregation for biomedical applications. European Journal of Pharmaceutics and Biopharmaceutics 2004;57:35-52.

Berger J, Reist M, Mayer JM, Felt O, Peppas NA, Gurny R. Structure and interactions in covalently and ionically crosslinked chitosan hydrogels for biomedical applications. European Journal of Pharmaceutics and Biopharmaceutics 2004;57:19-34.

Berrada M, Serreqi A, Dabbarh F, Owusu A, Gupta A, Lehnert S. Novel non-toxic camptothecin formulation for cancer chemotherapy. Biomaterials 2005;26:2115-2120.

Brandes AA, Tosoni A, Franceschi E, Reni M, Gatta G, Vecht C. Glioblastoma in adults. Critical Reviews in Oncology/Hematology 2008;67:139-152.

Brem H, Gabikian P. Biodegradable polymer implants to treat brain tumors. Journal of Controlled Release 2001;74:63-67.

Brem H, Lawson HC. The Development of New Brain Tumor Therapy Utilizing the Local and Sustained Delivery of Chemotherapeutic Agents from Biodegradable Polymers. Cancer 1999;86(2): 197-199.

Brenner M, Degitz K, Besch R, Berking C. Differential expression of melanomaassociated growth factors in keratinocytes and fibroblasts by ultraviolet A and ultraviolet B radiation. British Journal of Dermatology 2005;153:733-739.

Burstein HJ, Ramirez MJ, Petros WP, Clarke KD, Warmuth MA, Marcom PK, et al. Phase I study of Doxil and vinorelbine in metastatic breast cancer. Annals of Oncology 1999;10:1113-1116.

Castro DJ, Sridhar KS, Garewal HS, Mills GM, Wenig BL, Orenberg EK, et al. Intratumoral cisplatin/epinephrine gel in advanced head and neck cancer: A multicenter, randomized, doubleblind, phase III study in North America. Head \& Neck 2003;25:717731.

Chatelet C, Damour O, Domard A. Influence of the degree of acetylation on some biological properties of chitosan films. Biomaterials 2001;22:261-268. 
Chen FA, Kuriakose MA, Zhou MX, DeLacure MD, Dunn RL. Biodegradable polymermediated intratumoral delivery of cisplatin for treatment of human head and neck squamous cell carcinoma in a chimeric mouse model. Head \& Neck 2003;25:554-560.

Chen J, Freeman A, Liu J, Dai Q, Lee RM. The apoptotic effect of HA14-1, a Bcl-2interacting small molecular compound, requires Bax translocation and is enhanced by PK11195. Molecular Cancer Therapy 2002;1:981-987.

Chenite A, Buschmann M, Wang D, Chaput C, Kandani N. Rheological characterization of thermogelling chitosan/glycerolphosphate solutions. Carbohydrate Polymers 2001;46:39-47.

Chenite A, Chaput C, Wang D, Combes C, Buschmann MD, Selmani A, et al. Novel injectable neutral solutions of chitosan form biodegradable gels in situ. Biomaterials 2000;21:2155-2161.

Cho J, Heuzey MC, Begin A, Carreau PJ. Physical gelation of chitosan in the Presence of $\beta$-glycerophosphate: The effect of temperature. Biomacromolecules 2005;6:3267-3275.

Cho J, Heuzey MC, Begin A, Carreau PJ. Chitosan and glycerophosphate concentration dependence of solution behaviour and gel point using small amplitude oscillatory rheometry. Food Hydrocolloids 2006;20:936-945.

Cho K, Wang X, Nie S, Chen Z, Shin DM. Therapeutic Nanoparticles for Drug Delivery in Cancer. Clinical Cancer Research 2008;14(5): 1310-1316.

Cokgor I, Friedman AH, Friedman HS. Gliomas. European Journal of Cancer 1998;34:12:1910-1918.

Crompton KE, Goud JD, Bellamkond RV, Gengenbach TR, Finkelstein DI, Forsythe JS, et al. Polylysine-functionalised thermoresponsive chitosan hydrogel for neural tissue engineering. Biomaterials 2007;28: 441-449.

Dang W, Daviau T, Brem H. Morphological characterization of polyanhydride biodegradable implant Gliadel during in vitro and in vivo erosion using scanning electron microscopy. Pharmaceutical Research 1996;13(5):683-691.

Dang W, Daviau T, Ying P, Zhao Y, Nowotmk D, Brern H. Effects of GLIADEL ${ }^{\circledR}$ wafer initial molecular weight on the erosion of wafer and release of BCNU. Journal of Controlled Release 1996;42:83-92.

Dash AK, Cudworth GC. Therapeutic applications of implantable drug delivery systems. Journal of Pharmacological and Toxicological Methods 1998;40:1-12. 
Day AJ, Williamson G.. Biomarkers for exposure to dietary flavonoids: a review of the current evidence for identification of quercetin glycosides in plasma. British Journal of. Nutrition 2001;86(1):S105-S110.

Demierre MF. What about chemoprevention for melanoma? Current Opinion in Oncology 2006;18:180-184.

Dhanikula AB, Panchagnula R. Development and characterization of biodegradable chitosan films for local delivery of paclitaxel. American Association of Pharmaceutical Scientists Journal 2004;6:1-12.

Dixit R, Gold B. Inhibition of N-methyl-N-nitrosourea-induced mutagenicity and DNA methylation by ellagic acid. Biochemistry 1986;83:8039-8043.

Drixler TA, Rinkes IH, Ritchie ED, Vroonhoven TJ, Gebbink MF, Voest EE. Continuous administration of angiostatin inhibits accelerated growth of colorectal liver metastases after partial hepatectomy. Cancer Research 2000;60:1761-1765.

Dubin-Thaler BJ, Giannone G, Döbereiner HG, Sheetz MP. Nanometer analysis of cell spreading on matrix-coated surfaces reveals two distinct cell states and STEPs. Biophysics Journal. 2004;86(3):1794-1806.

Edderkaoui M, Odinokova I, Ohno I, Gukovsky I, Pandol SJ, Gukovskaya A. Ellagic acid induces apoptosis through inhibition of nuclear factor $\mathrm{kB}$ in pancreatic cells. World Journal of Gastroenterology 2008;14(23):3672-3680.

Elzinga EJ, Sparks DL. Phosphate adsorption onto hematite: An in situ ATR-FTIR investigation of the effects of $\mathrm{pH}$ and loading level on the mode of phosphate surface complexation. Journal of Colloid and Interface Science 2007; 308(1):53-70.

Ewend MG, Williams JA, Tabassi K, Tyler BM, Babel KM, Brem H, et al. Local delivery of chemotherapy and concurrent external beam radiotherapy prolongs survival in metastatic brain tumor models. Cancer Research 1996;56:5217-5223.

Feldman KS. Recent progress in ellagitannin chemistry. Phytochemistry $2005 ; 66: 1984-$ 2000.

Fialho SL, Cunha A. Manufacturing techniques of biodegradable implants intended for intraocular application. Drug Deliver 2005;12:109-116.

Findlay DM, Raggatt LJ, Bouralexis S, Hay S, Atkins GJ. Calcitonin decreases the adherence and survival of HEK-293 cells by a caspase-independent mechanism. Journal of Endocrinology 2002;175:715-725.

Freier T, Koh HS, Kazazian K, Shoichet MS. Controlling cell adhesion and degradation of chitosan films by N-acetylation. Biomaterials 2005;26:5872-5878. 
Gartel AL, Tyner AL. The role of the cyclin-dependent kinase inhibitor p21 in apoptosis. Molecular Cancer Therapy 2002;1:639-649.

Giussani C, Carrabba G, Pluderi M, Lucini V, Pannacci M, Caronzolo D, et al. Local intracerebral delivery of endogenous inhibitors by osmotic minipumps effectively suppresses glioma growth in vivo. Cancer Research 2003;63:2499-2505.

Gopalakrishna R, Gundimeda U. Antioxidant regulation of protein kinase $\mathrm{C}$ in cancer prevention. Journal of Nutrition 2002;132:3819-3823.

Gopferich A, Tessmar J. Polyanhydride degradation and erosion. Advanced Drug Delivery Reviews 2002;54:911-931. Journal of Controlled Release 2002;81:235-249.

Gorochovceva N, Makusuka R. Synthesis and study of water-soluble chitosan-Opoly(ethylene glycol) graft copolymers. European Polymer Journal 2004;40:685-91.

Graham CA, Cloughesy TF. Brain tumor treatment: Chemotherapy and other new developments. Seminars in Oncology Nursing 2004;20(4):260-272.

Groothuis DR. The blood-brain and blood-tumor barriers: A review of strategies for increasing drug delivery. Neuro-Oncology 2000;2:45-59.

Gunatillake PA, Adhikari R. Biodegradable synthetic polymers for tissue engineering. European Cells and Materials 2003;5:1-16.

Gutowska A, Jeong B, Jasionowski M. Injectable gels for tissue engineering. The Anatomical Record 2001;263:342-349.

Hammoud DA, Belden CJ, Ho AC, Dal Pan GJ, Brem H, Pomp MG, et al. The surgical bed after BCNU polymer wafer placement for recurrent glioma: Serial assessment on CT and MR imaging. American Journal of Roentgenology 2003;180:1469-1475.

Han X, Shen T, Lou H. Dietary polyphenols and their biological significance. International Journal of Molecular Sciences 2007;8:950-988.

Hanes J, Chiba M, Langer R. Synthesis and characterization of degradable anhydride-coimide terpolymers containing trimellitylimido-L-tyrosine: Novel polymers for drug delivery. Macromolecules 1996;29:5279-5287.

Harper E, Dang W, Lapidus RG, Garver RI. Enhanced efficacy of a novel controlled release paclitaxel formulation (Paclimer delivery system) for local-regional therapy of lung cancer tumor nodules in mice. Clinical Cancer Research 1999;5:4242-4248.

Heller J, Barr J, Ng SY, Abdellauoi KS, Gurny R. Poly(ortho esters): synthesis, characterization, properties and uses. Advanced Drug Delivery Reviews 2002;54:10151039. 
Heller J, Barr J, Ng SY, Shen HR, Abdellaoui KS, Gurny R, et al. Poly(ortho esters): their development and some recent applications. European Journal of Pharmaceutics and Biopharmaceutics 2000;50:121-128.

Hersey P. Apoptosis and melanoma: How new insights are effecting the development of new therapies for melanoma. Current Opinion in Oncology 2006;18:189-196.

Hoemann CD, Chenite A, Sun J, Hurtig M, Serreqi A, Buschmann MD, et al. Cytocompatible gel formation of chitosan-glycerol phosphate solutions supplemented with hydroxyl ethyl cellulose is due to the presence of glyoxal. Journal of Biomedical Materials Research Part A 2007;56(2):521-529.

Hoemann CD, Sun J, MSC AL, McKee MD, Buschmann MD. Tissue engineering of cartilage using an injectable and adhesive chitosan-based cell-delivery vehicle. OsteoArthritis and Cartilage 2005;13:318-329.

Hong Y, Song H, Gong Y, Mao Z, Gao C, Shen J. Covalently crosslinked chitosan hydrogel: Properties of in vitro degradation and chondrocyte encapsulation. Acta Biomaterialia 2007;3:23-31.

Hussein MR, Haemel AK, Wood GS. Apoptosis and melanoma: Molecular mechanisms. Journal of Pathology 2003;199:275-288. (Review article).

Iliescu M, Hoemann CD, Shive MS, Chenite A, Buschmann MD. Ultrastructure of hybrid chitosan-glycerol phosphate blood clots by environmental scanning electron microscopy. Microscopy Research and Technique 2008;71:236-247.

Isoniemi H, Appelberg J, Nilsson CG, Makela P, Risteli J, Hockerstedt K. Transdermal oestrogen therapy protects postmenopausal liver transplant women from osteoporosis. A 2-year follow-up study. Journal of Hepatology 2001;34:299-305.

Jain RA. The manufacturing techniques of various drug loaded biodegradable poly(lactide-co-glycolide) (PLGA) devices. Biomaterials 2000;21:2475-2490.

Jain RK. Delivery of molecular and cellular medicine to solid tumors. Advanced Drug Delivery Reviews 2001;46:149-168.

Jarry C, Leroux JC, Haeck J, Chaput C. Irradiating or autoclaving chitosan/polyol solutions: Effect on thermogelling chitosan-b- glycerophosphate systems. Chemical \& Pharmaceutical Bulletin. 2002;50(10):1335-1340.

Jaworska M, Sakurai K, Gaudon P, Guibal E. Influence of chitosan characteristics on polymer properties I. Crystallographic properties. Polymer International 2003;52:198-205.

Jayakumar R, Prabaharan M, Reis RL, Mano JF. Graft copolymerized chitosan-present status and applications. Carbohydrate Polymer 2005;62:142-215. 
Jeong B, Bae YH, Kim SW. Drug release from biodegradable injectable thermosensitive hydrogel of PEG-PLGA-PEG triblock copolymers. Journal of Controlled Release 2000;63:155-163.

Katti DS, Lakshmi S, Langer R, Laurencin CT. Toxicity, biodegradation and elimination of polyanhydrides. Advanced Drug Delivery Reviews 2002;54:933-961.

Keskar V, Mohanty PS, Gemeinhart EJ, Gemeinhart RA. Cervical cancer treatment with a locally insertable controlled release delivery system. Journal of Controlled Release 2006; 115:280-288.

Khan N, Afaq F, Mukhtar H. Apoptosis by dietary factors: the suicide solution for delaying cancer growth. Carcinogenesis 2007;28(2):233-239.

Khan TA, Peh KK, Ching HS. Reporting degree of deacetylation values of chitosan: the influence of analytical methods. Journal of Pharm Pharmaceut Sci 2002;5(3):205-212.

Kim HJ, Park HJ, Park WS, Bae YM. CD43 cross-linking increases the Fas-induced apoptosis through induction of Fas aggregation in Jurkat T-cells. Experimental \& molecular medicine 2006;38:357-363.

Kim S, Gaber MW, Zawaski JA, Zhang F, Richardson M, Zhang XA, Yang Y. The inhibition of glioma growth in vitro and in vivo by a chitosan/ellagic acid composite biomaterial. Biomaterials 2009;30(27):4743-4751.

Kim S, Liu Y, Gaber MW, Bumgardner JD, Haggard WO, Yang Y. Development of chitosan-ellagic acid films as a local drug delivery system to induce apoptotic death of human melanoma cells. Journal of Biomedical Materials Research Part B: Applied Biomaterials 2009;90B(1):145-155.

Kim SA, Kim YC, Kim SW, Lee SH, Min JJ, Ahn SG et al. Antitumor Activity of Novel Indirubin Derivatives in Rat Tumor Model. Journal of Clinical Cancer Research 2007;13(1):253-259.

Kisker O, Becker CM, Prox D, Fannon M, Amato R, Flynn E, et al. Continuous administration of endostatin by intraperitoneally implanted osmotic pump improves the efficacy and potency of therapy in a mouse xenograft tumor model. Cancer Research 2001;61:7669-7674.

Kokufuta E. Polyelectrolyte gel transitions: experimental aspects of charge inhomogeneity in the swelling and segmental attractions in the shrinking. Langmuir 2005;21(22):10004-10015.

Kubota Y, Kleinman HK, Martin GR, Lawley TJ. Role of Laminin and Basement Membrane in the Morphological Differentiation of Human Endothelial Cells into Capillary-like Structures. The Journal of Cell Biology 1988;107:1589-1598. 
Kumar MN. A review of chitin and chitosan applications. Reactive \& Functional Polymers 2000;46:1-27.

Kuypers FA, Lewis RA, Hua M, Schott MA, Discher D, Lubin BH, et al. Detection of altered membrane phospholipid asymmetry in subpopulations of human red blood cells using fluorescently labeled annexin V. Blood 1996;87:1179-1187.

Labrecque L, Lamy S, Chapus A, Mihoubi S, Durocher Y, Cass B, et al. Combined inhibition of PDGF and VEGF receptors by ellagic acid, a dietary-derived phenolic compound. Carcinogenesis 2005;26(4):821-826.

Larrosa M, Tomas-Barberan FA, Espin JC. The dietary hydrolysable tannin punicalagin releases ellagic acid that induces apoptosis in human colon adenocarcinoma Caco-2 cells by using the mitochondrial pathway. Journal of Nutritional Biochemistry 2006;17:611625.

Laura AK, Mark AM, Charlotte M, Peter SC, Jerry L, Ashok G et al. Chemoprevention of Esophageal Tumorigenesis by Dietary Administration of Lyophilized Black Raspberries. Cancer Research 2001;61:6112-6119.

Lazze MC, Savio M, Pizzala R, Cazzalini O, Perucca P, Bianchi L, et al. Anthocyanins induce cell cycle perturbations and apoptosis in different human cell lines. Carcinogenesis 2004;25:1427-1433.

Lee KY, Alsberg E, Mooney DJ. Degradable and injectable poly(aldehyde guluronate) hydrogels for bone tissue engineering. Materials Research Part A 2001;83A (2):228-233.

Lee YJ, Kuo HC, Chu CY, Wang CJ, Lin WC, Tseng TH. Involvement of tumor suppressor protein $\mathrm{p} 53$ and $\mathrm{p} 38$ MAPK in caffeic acid phenethyl ester-induced apoptosis of C6 glioma cells. Biochemical Pharmacology 2003;66(12):2281-2289.

Li J, Du Y, Liang H. Influence of molecular parameters on the degradation of chitosan by a commercial enzyme. Polymer Degradation and Stability 2007;92:515-524.

Li Q, Pan PY, Gu P, Xu D, Chen SH. Role of Immature Myeloid Gr-1+ Cells in the Development of Antitumor Immunity. CANCER RESEARCH 2004;64:1130-1139.

Liu L, Sheardown H. Glucose permeable poly (dimethyl siloxane) poly ( $\mathrm{N}$-isopropyl acrylamide) interpenetrating networks as ophthalmic biomaterials. Biomaterials 2005;26: 233-244.

Loo G. Redox-sensitive mechanisms of phytochemical-mediated inhibition of cancer cell proliferation (review). Journal of Nutritional Biochemistry 2003;14(2):64-73.

Losso JN, Bansode RR, Trappey A, Bawadi HA, Truax R. In vitro anti-proliferative activities of ellagic acid. The Journal of Nutritional Biochemistry 2004;15:672-678. 
Lu Z, Yeh TK, Tsai M, Au JL, Wientjes MG. Paclitaxelloaded gelatin nanoparticles for intravesical bladder cancer therapy clinical. Cancer Research 2004;10:7677-7684.

Lyass O, Uziely B, Ben R, Heshing DT, Lotem M, Brufman G, et al. Correlation of toxicity with pharmacokinetics of Pegylated Liposomal Doxorubicin (Doxil) in metastatic breast carcinoma. Cancer 2000;89:1037-1047.

Manach C, Morand C, Gil-Izquierdo A, Bouteloup-Demange C, Remesy C. Bioavailability in humans of the flavanones hesperidin and narirutin after the ingestion of two doses of orange juice. European Journal of Clinical Nutrition 2003;57:235-242.

Marina NM, Cochrane D, Harney E, Zomorodi K, Blaney S, Winick N, et al. Dose escalation and pharmacokinetics of Pegylated Liposomal Doxorubicin (Doxil) in children with solid tumors: A pediatric oncology group study. Clinical Cancer Research 2002;8: 413-418.

Maurer N, Fenske DB, Cullis PR. Developments in liposomal drug delivery systems. Expert Opinion on Biological Therapy 2001;1(5): 1-25.

Menei P, Jadaud E, Faisant N, Michalak S, Fournier D, Delhaye M, et al. Stereotaxic implantation of 5-Fluorouracil-releasing microspheres in malignant glioma. Cancer 2004;100(2):405-410.

Menei P, Venier MC, Gamelin E, Saint-Andre JP, Hayek G, Jadaud E, et al. Local and sustained delivery of 5-Fluorouracil from biodegradable microspheres for the radiosensitization of glioblastoma. Cancer 1999;15(86):325-330.

Mertens-Talcott SU, Bomser JA, Romero C, Talcott ST, Percival SS. Ellagic acid potentiates the effect of quercetin on $\mathrm{p} 21 \mathrm{waf1} / \mathrm{cip} 1$, p53, and MAP-kinases without affecting intracellular generation of reactive oxygen species in vitro. Journal of Nutrition 2005; 135:609-614.

Mertens-Talcott SU, Talcott ST, Percival SS. Low concentrations of quercetin and ellagic acid synergistically influence proliferation, cytotoxicity and apoptosis in MOLT-4 human leukemia cells. J Nutr 2003;133:2669-2674.

Mi FL, Shyu SS, Chen CT, Lai JY. Adsorption of indomethacin onto chemically modified chitosan beads. Polymer 2002;43:757-765.

Molinaro G, Leroux JC, Damas J, Adam A. Biocompatibility of thermo-sensitive chitosan-based hydrogels: an in vivo experimental approach to injectable biomaterials. Biomaterials 2002;23:2717-2722.

Moses MA, Brem H, and Langer R, Advancing the field of drug delivery: Taking aim at cancer. Cancer Cell 2003;4:337-341. 
Mullen W, Yokota T, Lean ME, Crozier A. Analysis of ellagitannins and conjugates of ellagic acid and quercetin in raspberry fruits by LC-MSn. Phytochemistry. 2003;64:617624.

Muzzarelli R. Human enzymatic activities related to the therapeutic administration of chitin derivatives. Cellular and Molecular Life Sciences. 1997;53:131-140.

Nair LS, Starnes T, Ko JK, Laurencin CT. Development of injectable thermogelling chitosan - inorganic phosphate solutions for biomedical applications. Biomacromolecules 2007;8:3779-3785.

Narayanan BA, Geoffroy O,Willingham MC, Re GG, Nixon DW. p53/p21(WAF1/CIP1) expression and its possible role in G1 arrest and apoptosis in ellagic acid treated cancer cells. Cancer Letters 1999;136:215-221.

Neamnark A, Sanchavanakit N, Pavasant P, Bunaprasert T, Supaphol P, Rujiravanit R. In vitro biocompatibility evaluations of hexanoyl chitosan film. Carbohydrate Polymer 2007;68:166-172.

Oak MH, Bedoui JE, Schini-Kerth VB. Antiangiogenic properties of natural polyphenols from red wine and green tea. Journal of Nutritional Biochemistry 2005;16:1-8.

Olivi A, Grossman SA, Tatter S, Barker F, Judy K, Olsen J, et al. Dose Escalation of Carmustine in Surgically Implanted Polymers in Patients With Recurrent Malignant Glioma: A New Approaches to Brain Tumor Therapy CNS Consortium Trial. Journal of Clinical Oncology 2003;21(9):1845-1849.

Pangburn SH, Trescony PV, Heller J. Lysozyme degradation of partially deacetylated chitin, its films and hydrogels. Biomaterials 1982;3:105-108.

Park H, Park K. Biocompatibility issues of implantable drug delivery systems. Pharmaceutical Research 1996;13(12):1770-1776.

Perry J, Chambers A, Spithoff K, Laperriere N. Gliadel wafers in the treatment of malignant glioma: a systematic review. Current Oncology 2005;14(5):205-214.

Pillai CKS, Paul W, Sharma CP. Chitin and chitosan polymers: Chemistry, solubility and fiber formation. Progress in Polymer Science 2009;34:641-678.

Pinto M, Lajolo FM, Genovese MI. Bioactive compounds and quantification of total ellagic acid in strawberries. Food Chemistry 2008;107:1629-1635.

Prashanth KH, Tharanathan RN. Depolymerized products of chitosan as potent inhibitors of tumor-induced angiogenesis. Biochimica et Biophysica Acta 2005;1722:22-29. 
Purow B, Fine HA. Antiangiogenic therapy for primary and metastatic brain tumors. Hematology/Oncology Clinics of North America 2004;18(5):1161-1181.

Qu X, WirseN A, Albertsson AC. Synthesis and characterization of $\mathrm{pH}$-sensitive hydrogels based on chitosan and D,L-Lactic acid. Journal of Applied Polymer Science 1999;74:3193-3202.

Rahman I, Biswas SK, Kirkham PA. Regulation of inflammation and redox signaling by dietary polyphenols. Biochemical Pharmacology 2006;72:1439-1452.

Ravi Kumar MN, Hudson SM. Chitosan. Encyclopedia of biomaterials and biomedical engineering 2006: 310-323.

Ren D, Yi H, Wang W, Ma X. The enzymatic degradation and swelling properties of chitosan matrices with different degrees of $\mathrm{N}$-acetylation. Carbohydrate Research 2005;340:2403-2410.

Rice-Evans CA, Miller NJ, Paganga G. Structure-antioxidant activity relationships of flavonoids and phenolic acids. Free Radical Biology \& Medicine 1996;20(7):933-956.

Rieber MS, Zangemeister-Wittke U, Rieber M. p53-independent induction of apoptosis in human melanoma cells by a bcl-2/bcl-xL bispecific antisense oligonucleotide. Clinical Cancer Research 2001;7:1446-1451.

Rose PG.. Pegylated Liposomal Doxorubicin: Optimizing the dosing schedule in ovarian cancer. The Oncologist 2005; 10:205-214.

Roughley P, Hoemann C, DesRosiers E, Mwale F, Antoniou J, Alini M. The potential of chitosan-based gels containing intervertebral disc cells for nucleus pulposus supplementation. Biomaterials 2006;27:388-396.

Ruel-Gariepy E, Chenite A, Chaput C, Guirguis S, Leroux JC. Characterization of thermosensitive chitosan gels for the sustained delivery of drugs. International Journal of Pharmaceutics 2000;203:89-98.

Ruel-Gariepy E, Shive M, Bichara A, Berrada M, Garrec DL, Chenite A, Leroux JC. A thermo-sensitive chitosan-based hydrogel for the local delivery of paclitaxel. European Journal of Pharmaceutics and Biopharmaceutics 2004;57:53-63.

Rundhaug JE. Matrix Metalloproteinases, Angiogenesis, and Cancer. Clinical Cancer Research 2003;9:551-554.

Sashiwa H, Aiba S. Chemically modified chitin and chitosan as biomaterials. Prog Polym Sci 2004;29:887-908. 
Scalbert A, Williamson G. Dietary intake and bioavailability of polyphenols. The Journal of Nutrition 2000;130:2073S-2085S.

Schuler M, Bossy-Wetzel E, Goldstein JC, Fitzgerald P, Green DR. p53 induces apoptosis by caspase activation through mitochondrial cytochrome $\mathrm{c}$ release. Journal of Biological Chemistry 2000;,275(10):7337-7342.

Schwartz LH, Colville JAC, Ginsberg MS, Wang L, Mazumdar M, Kalaigian K et al. Measuring tumor response and shape change on CT: esophageal cancer as a paradigm. Annals of Oncology 2006;17:1018-1023.

Schwartz LH, Ginsberg MS, DeCorato D, Rothenberg LN, Einstein S, Kijewski P et al. Evaluation of Tumor Measurements in Oncology: Use of Film-Based and Electronic Techniques. Journal of Clinical Oncology 2000;18(10):2179-2184.

Seeram NP, Henning SM, Zhang YJ, Suchard M, Li Z, Heber D. Pomegranate juice ellagitannin metabolites are present in human plasma and some persist in urine for up to 48 hours. The Journal of Nutrition 2006;136:2481-2485.

Seeram NP, Lee R, Heber D. Bioavailability of ellagic acid in human plasma after consumption of ellagitannins from pomegranate (Punica granatum L.) juice. Clinica Chimica Acta 2004;348:63-68.

Shukla M, Gupta K, Rasheed Z, Khan KA, Haqqi TM. Bioavailable constituents / metabolites of pomegranate (Punica granatum L) preferentially inhibit COX2 activity ex vivo and IL-1beta-induced PGE2 production in human chondrocytes in vitro. Journal of Inflammation 2008;5(9):1-10.

Silvia RM, Silvia GA, Coutinho OP, Mano JF, Reis RL. Preparation and characterisation in simulated body conditions of glutaraldehyde crosslinked chitosan membranes. Journal of materials science. Materials in medicine 2004;15:1105-1112.

Simmons A, Padsalgikar AD, Ferris LM, Warren LA. Biostability and biological performance of a PDMS-based polyurethane for controlled drug release. Biomaterials 2008;29:2987-2995.

Smith SH, Goldschmidt MH, McManus PM. A Comparative Review of Melanocytic Neoplasms. Veterinary Pathology. 2002;39:651-678.

Spencer JP, Chowrimootoo G, Choudhury R, Debnam ES, Srai SK, Rice-Evans C. The small intestine can both absorb and glucuronidate luminal flavonoids. FEBS Letters 1999 ;458:224-230.

Stoclet JC, Chataigneau T, Ndiaye M, Oak MH, Bedoui JE, Schini-Kerth VB, et al. Vascular protection by dietary polyphenols. European Journal Pharmacology 2004;500:299-313. 
Stokke B, Varum KM, Holme HK, Hjerde R, Smidsrod O. Sequence specificities for lysozyme depolymerization of partially N-acetylated chitosans. Canadian Journal of Chemistry 1995;73:1972-1981.

Sun Y, Oberley LW. Redox regulation of transcriptional activators. Free Radical Biology \& Medicine 1996;21(3):335-348.

Ta HT, Dass CR, Dunstan DE. Injectable chitosan hydrogels for localised cancer therapy. Journal of Controlled Release 2008;126: 205-216.

Ta HT, Han H, Larson I, Dass CR, Dunstan DE. Chitosan-dibasic orthophosphate hydrogel: A potential drug delivery system. International Journal of Pharmaceutics 2009;371:134-141.

Tangpasuthadol V, Pendharkar SM, Peterson RC, Kohn J. Hydrolytic degradation of tyrosine-derived polycarbonates, a class of new biomaterials. Part II: 3-yr study of polymeric devices. Biomaterials 2000;21:2379-2387.

Tien CL, Lacroix M, Ispas-Szabo P, Mateescu MA. N-acylated chitosan: Hydrophobic matrices for controlled drug release. Journal of Controlled Release 2003;93:1-13.

Tomihata K, Ikada Y. In vitro and in vivo degradation of films of chitin and its deacetylated derivatives. Biomaterials 1997;16:567-575.

Tonnesen P, Paoletti P, Gustavsson G, Russell MA, Saracci1 R, Gulsvik A. Higher dosage nicotine patches increase one-year smoking cessation rates: results from the European CEASE trial. European Respiratory Journal 1999; 13: 238-246.

Vekiari SA, Gordon MH, Garcia-Macias P, Labrinea H. Extraction and determination of ellagic acid content in chestnut bark and fruit. Food Chemistry 2008;110:1007-1011.

Vishu-Kumar AB, Gowda RL, Tharanathan RN. Non-specific depolymerization of chitosan by pronase and characterization of the resultant products. European Journal of Biochemistry 2004;271:713-723.

Vogi TJ, Engelmann K, Mack MG, Straub R, Zangos S, Eichler K, et al. CT-guided intratumoural administration of cisplatin/epinephrine gel for treatment of malignant liver tumours. British Journal of Cancer 2002;86:524-529.

Walter KA, Cahan MA, Gur A, Tyler B, Hilton J, Michael CO et al. Interstitial Taxol Delivered from a Biodegradable Polymer Implant against Experimental Malignant Glioma. Cancer Research 1994;54:2207-2212.

Wang PP, Frazier J, Brem H. Local drug delivery to the brain. Advanced Drug Delivery Reviews 2002;54:987-1013. 
Wang QZ, Chen XZ, Liu N, Wang SX, Liu CS, Meng XH, Liu CG. Protonation constants of chitosan with different molecular weight and degree of deacetylation. Carbohydrate Polymers 2006;65:194-201.

Wang T, Turhan M, Gunasekaran S. Selected properties of $\mathrm{pH}$-sensitive, biodegradable chitosan-poly(vinyl alcohol) hydrogel. Polymer International 2004;53:911-918.

Westphal M, Lamszus K. Intracavitary chemotherapy for glioblastoma: present status and future directions. Acta neurochirurgica. Supplement $2003 ; 88: 61-71$.

Whitley AC, Stoner GD, Darby MV, Walle T. Intestinal epithelial cell accumulation of the cancer preventive polyphenol ellagic acid dextensive binding to protein and DNA. Biochemical Pharmacology 2003;66:907-915.

Williamson G., Day AJ, PlumbGW, Couteau D. Human metabolic pathways of dietary flavonoids and cinnamates. Biochemical Society Transactions 2000;28:16-22.

Willingham MC. Cytochemical methods for the detection of apoptosis. J Histochem Cytochem 1999;47:1101-1109.

Withrow SJ, Liptak JM, Straw RC, Dernell WS, Jameson VJ, Douple EB, et al. Biodegradable cisplatin polymer in limb-sparing surgery for canine osteosarcoma. Annals of Surgical Oncology 2004;11:705-713.

Wu J, Su ZG, Ma GH. A thermo- and $\mathrm{pH}$-sensitive hydrogel composed of quaternized chitosan/glycerophosphate. International Journal of Pharmaceutics 2006;315: 1-11.

Wu J, Wei W, Wang LY, Su ZG, Ma GH. A thermosensitive hydrogel based on quaternized chitosan and poly (ethylene glycol) for nasal drug delivery system. Biomaterials 2007;28:2220-2232.

Yamaguchi Y, Takenaga M, Kitagawa A, Ogawa Y, Mizushima Y, Igarashi R. Insulinloaded biodegradable PLGA microcapsules: initial burst release controlled by hydrophilic additives. Journal of Controlled Release 2002;81:235-249.

Yoshifuji A, Noishiki Y, Wada M, Heux L, Kuga S. Esterification of $\beta$-chitin via intercalation by carboxylic anhydrides. Biomacromolecules 2006;7:2878-2881.

Yuan Y, Chesnutt BM, Utturkar G, Haggard WO, Yang Y, Ong JL, Bumgardner JD. The effect of cross-linking of chitosan microspheres with genipin on protein release. Carbohydrate Polymer 2007;68:561-567.

Zhang X, Wu D, Chu C. Synthesis and characterization of partially biodegradable, temperature and $\mathrm{pH}$ sensitive Dex-MA/PNIPAAm hydrogels. Biomaterials 2003;25:4719-4730. 
Zignani M, Einmahl S, Baeyens V, Varesio E, Veuthey JL, Gurny R, et al. A poly(ortho ester) designed for combined ocular delivery of dexamethasone sodium phosphate and 5fluorouracil: subconjunctival tolerance and in vitro release. European Journal of Pharmaceutics and Biopharmaceutics 2000;50:251-255. 


\section{VITA}

Sung Woo Kim was born in 1972 in South Korea. He attended the engineering school of the Sung Kyun Kwan University and earned his Bachelor of Science degree in Textile Engineering in 1998 and Master of Science degree in 2000. During his time at the Sung Kyun Kwan University, Mr. Kim also served in the army for 26 months as a fulfillment of obligation to South Korea. In January 2000, he started work with KOLON Chemical Research Center in South Korea as a researcher for a year. He entered the University of Southern California in 2002 and received his Master of Science degree in Biomedical Engineering in 2004. He enrolled in the joint graduate program in Biomedical

Engineering and Imaging at the University of Tennessee Health Science Center and the University of Memphis in 2005. Sung Woo Kim plans to continue pursuing his research interests in tissue engineering and drug delivery. 